\title{
Health, Fitness, Lifestyle and Physical Competency of School Children in Wales
}

\author{
Richard Paul Oliver Tyler
}

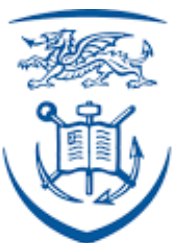

Swansea University

Prifysgol Abertawe

Submitted to Swansea University in fulfilment of the requirements for the Degree of Doctor of Philosophy

Swansea University

2018 


\begin{abstract}
Promoting physical activity, fitness and physical competence are effective preventative measures for a variety of health risks and non-communicable diseases. Identifying overall levels, trends and inequalities among children and young people is imperative to inform and deliver effective programmes and initiatives aimed at increasing physical activity, health-related fitness and physical competence levels. The current challenge is to identify the interrelationships between these factors and to develop new insight into their assessment and integration into physical activity frameworks and models. Therefore, the overarching aim of the thesis was to investigate the health, fitness, lifestyle and physical competency of school children.
\end{abstract}

Study 1 consolidated and translated surveillance data related to physical activity among children and young people in Wales in order to assign grades to physical activity/ inactivity behaviours and influences indicators. Studies 2 and 3 highlighted the secular changes and the socio-economic and sex differences, respectively, in selected healthrelated fitness measures. Taken together, Studies 1, 2 and 3 showed that physical activity behaviours and influences and overall health-related fitness levels were low, and inequalities existed across socio-economic status and sex. Given the importance of physical competence in children and young people, Study 4 summarised the development of a novel, dynamic measure of physical competence (Dragon Challenge) that incorporates fundamental, combined and complex movement skills. Study 4 revealed that the Dragon Challenge was a valid and reliable tool in 10-14 year old children, and Study 5 resulted in the first reported model that quantified the direct and indirect relationships between ecological factors on physical competence.

Overall this thesis advocates for a balance of physical activity (quantity and exposure) and fitness and physical competency (quality and outcome) in children and young people and can be used to inform the content and delivery of health education interventions and programmes. 


\section{Scientific Outputs}

\section{Publications}

Tyler, R., Mackintosh, K., Palmer, A., Jones, A., \& Stratton, G. (2015). Ten-Year Secular Changes in Selected Health and Fitness Parameters of 10-11 Years Old Swansea School Children - 2003-2013. Advances in Obesity, Weight Management \& Control, 3(5), 8-13. https://doi.org/10.15406/aowmc.2015.03.00072

Tyler, R., Mannello, M., Mattingley, R., Roberts, C., Sage, R., Taylor, S. R., .. Stratton, G. (2016). Results From Wales' 2016 Report Card on Physical Activity for Children and Youth: Is Wales Turning the Tide on Children's Inactivity? Journal of Physical Activity and Health, 13(11 Suppl 2), S330-S336. https://doi.org/10.1123/jpah.2016-0309

Tyler, R., Foweather, L., Mackintosh, K. A., \& Stratton, G. (2018). A Dynamic Assessment of Children's Physical Competence. Medicine \& Science in Sports \& Exercise, 50(12), 2474-2487. https://doi.org/10.1249/MSS.0000000000001739

Tyler, R., Mackintosh, K. A., Foweather, L., Edwards, L. \& Stratton, G. (2018). Youth Physical Competence Promotion Model: A Quantitative Investigation into Modifiable Factors. Scandinavian Journal of Medicine \& Science in Sports (under review).

Tyler, R., Mackintosh, K. A., Spacey, H. L. \& Stratton, G. (2018). A Cross-Sectional Study on the Socio-economic and Sex Differences in Health-Related Fitness Measures in School Children. Journal of Sports Sciences (under review).

\section{Presentations}

Tyler, R., Spacey, H. L., Stratton, G. Dragon Multi-skills and Sport: The Dragon Challenge, International Physical Literacy Association (IPLA) Conference, Cardiff, UK. June 2018. Oral Presentation

Tyler, R., Foweather, L., Mackintosh, K.A., Stratton, G. Validity and reliability of the Dragon Challenge v1.0 assessment of physical/motor competence, United Kingdom \& Ireland Motor Competence Network Meeting, Coventry, UK. January 2018. Oral Presentation 
Tyler, R., Edwards, L.C. International Project on Physical Activity: Active Healthy Kids Wales Report Card, National Centre for Population Health and Wellbeing Research (NCPHWR), Public Health Wales Annual Conference, Cardiff, UK. October 2017. Oral Presentation.

Tyler, R., Mackintosh, K.A., Foweather, L., Stratton, G. The validity and reliability of the Dragon Challenge V1.0 in measuring physical competency in children aged 8-14 years, 1st Annual Pan-Wales Postgraduate Conference in Sport and Exercise Sciences, Swansea, UK. April 2017. Oral Presentation.

Tyler, R., Mannello, M., Mattingley, R., Roberts, C., Sage, R., Taylor, S.R., Ward, M., Williams, S., Stratton, G. Results from the Active Healthy Kids Wales 2016 Report Card on Physical Activity for Children and Youth, International Society of Physical Activity and Health (ISPAH), International Congress on Physical Activity and Public Health, Bangkok, Thailand. November 2016. Poster Presentation.

Tyler, R., Mannello, M., Mattingley, R., Roberts, C., Sage, R., Taylor, S.R., Ward, M., Williams, S., Stratton, G. Results from the Active Healthy Kids Wales 2016 Report Card on Physical Activity for Children and Youth, Active Healthy Kids Wales Report Card Launch, Swansea, Wales, UK. July 2016. Oral Presentation.

Tyler, R., Mackintosh, K.A., McNarry, M., Foweather, L., Rosenberg, M., Stratton, G. The odds of being physically competent according to fitness and weight status in 9-11 year old girls and boys, The British Association of Sport and Exercise Sciences (BASES) Student Conference, Bangor, Wales, UK. March 2016. Oral Presentation.

Tyler, R., Mackintosh, K.A., McNarry, M., Foweather, L., Rosenberg, M., Stratton, G. The odds of being physically competent according to fitness and weight status in 9-11 year old girls and boys, Applied Sport Technology Exercise and Medicine Research Centre (A-STEM) Postgraduate Conference, Swansea, Wales, UK. March 2016. Oral Presentation.

Tyler, R., Christian, D., Todd, C., Brophy, S., Stratton, G. HAPPEN: Health Action Planning through a Primary Education Network - Utilisation of data obtained through the Swan-Linx Project, School Health Network, Swansea, Wales, UK. November 2015. Oral Presentation. 
Tyler, R., Mackintosh, K.A., Palmer, A., Jones, A., Stratton, G. Changes in selected health \& fitness parameters of Year 6 - 2003-2013 Swansea school children, Applied Sport Technology Exercise and Medicine Research Centre (A-STEM) Postgraduate Conference, Swansea, Wales, UK. March 2015. Oral Presentation.

Tyler, R., Stratton, G. Swan-Linx Research, Indonesian Delegate Seminar, Swansea, Wales, UK. December 2014. Oral Presentation. 


\section{Declarations and Statements}

1. I, Richard Paul Oliver Tyler, hereby declares that the work presented in this thesis has not previously been accepted in substance for any degree and is not being concurrently submitted in candidature for any degree.

2. I, Richard Paul Oliver Tyler, hereby declares that the thesis is the result of my own investigations, except where otherwise stated and that other sources are acknowledged by footnotes giving explicit references and that a bibliography is appended.

3. I, Richard Paul Oliver Tyler hereby gives consent for the thesis, if accepted, to be available for photocopying and for inter-library loan, and for the title and summary to be made available to outside organisations.

Signed: (candidate)

Date: 


\section{Contents}

Abstract

Scientific Outputs $v$

Declarations and Statements vii

Acknowledgements $\quad$ xvi

$\begin{array}{ll}\text { List of Tables } & \text { xvii }\end{array}$

$\begin{array}{ll}\text { List of Figures } & \text { xviii }\end{array}$

Abbreviations $\quad$ xx

1 Introduction 1

1.1 Rationale and background $\ldots \ldots \ldots \ldots 1$

1.2 Problem statement . . . . . . . . . . . . . . . . 6

1.3 Thesis aims ....................... 6

2 Literature Review 9

2.1 Physical Activity . . . . . . . . . . . . . . . . . 9 9

2.2 UK Physical Activity Guidelines . . . . . . . . . . . . . . 10

2.3 Physical Activity and Sedentary Behaviours in Children and Young People . . . . . . . . . . . . . . . . . . . . 11

2.4 Ecological Models . . . . . . . . . . . . . . . . . . . . 12

2.5 Monitoring Physical Activity . . . . . . . . . . . . . . . 15

2.6 Product/Outcome Measure of Physical Activity - Physical Fitness . . 16

2.6.1 Cardiorespiratory Fitness . . . . . . . . . . . . . . 18

2.6.2 Muscular Strength . . . . . . . . . . . . . . . . 20

2.6 .3 Body Mass Index . . . . . . . . . . . . . . . . 21

2.6 .4 Biological Maturation _. . . . . . . . . . . 23

2.7 Product/Outcome Measure of Physical Activity - Physical Literacy/

Physical Competence . . . . . . . . . . . . . . . . 24

2.7.1 Measurement of Physical Competence . . . . . . . . . . 27

3 General methodology 31

3.1 Ethical Approval . . . . . . . . . . . . . . . . . . 31

3.2 Instruments and Procedures . . . . . . . . . . . . . . . 31

3.2.1 Swan-Linx Fitness Fun Day: Field-Based Fitness Measures . 31 
3.2.2 Child Health and Activity Tool: Online Questionnaire . . . . 33

3.2.3 The Dragon Challenge: Physical Competence Measure . . . . 33

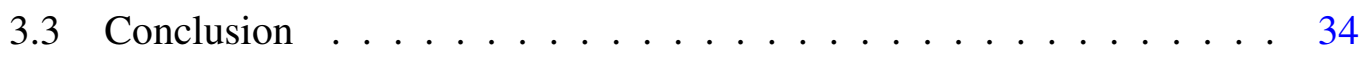

$\begin{array}{ll}\text { Thesis Map } & 35\end{array}$

4 Study $1 \quad 37$

4.1 Results from the Active Healthy Kids Wales 2016 Report Card on Physical Activity for Children and Youth: "Is Wales Turning the Tide on Children's Inactivity?" . . . . . . . . . . . . . . . . . . . 37

4.2 Background . . . . . . . . . . . . . . . 37

4.3 Methods . . . . . . . . . . . . . . . . . . . 38

4.4 Results . . . . . . . . . . . . . . . . . . . . . 41

4.4.1 Overall Physical Activity Levels . . . . . . . . . . . . . . . . 42

4.4.2 Organised Sport Participation . . . . . . . . . . . . . . . . . . . . . . . . . . 43

4.4 .3 Outdoor and Active play . . . . . . . . . . . . . . 43

4.4.4 Active Transportation . . . . . . . . . . . . . . . . . . 44

4.4.5 Sedentary Behaviours . . . . . . . . . . . . 44

4.4 .6 Physical Literacy . . . . . . . . . . . . . . . . 45

4.4.7 Family and Peer Influence . . . . . . . . . . . . . . . 45

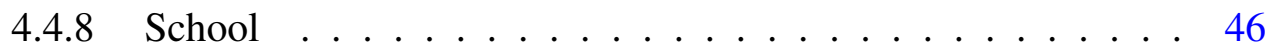

4.4.9 Community and the Built Environment . . . . . . . . . . 46

4.4.10 National Government Policy, Strategies and Investments . . . 47

4.5 Discussion . . . . . . . . . . . . . . . . . . . . . 48

4.5.1 Research Gaps . . . . . . . . . . . . . . . . . . . . 49

4.5.2 Recommendations to improve the grades . . . . . . . . . 50

4.5 .3 Future Directions . . . . . . . . . . . . . . . . . 51

4.6 Conclusion ........................ 51

4.7 Summary: Study $1 \ldots \ldots \ldots 52$

$\begin{array}{lc}\text { Thesis Map } & 53\end{array}$

5 Study 2

5.1 Ten-year Secular Changes in Selected Health and Fitness Parameters of 10-11 Years Old Swansea School Children - 2003 - 2013 . . . . 55

5.2 Introduction . . . . . . . . . . . . . . . . . . 55

5.3 Methods . . . . . . . . . . . . . . . . 56

5.3 .1 Participants and Settings . . . . . . . . . . . 57

5.3 .2 Instruments and Procedures _ . . . . . . . . . . . . . 57 
5.3 .3 Design and Analysis . . . . . . . . . . . . . . 58

5.4 Results . . . . . . . . . . . . . . . . . . . 58

$5.4 .1 \quad$ BMI z-scores . . . . . . . . . . . . . . . . . . . 59

5.4.2 Prevalence of overweight and obesity . . . . . . . . . 60

5.4 .3 Grip strength . . . . . . . . . . . . . . . . . . 60

5.5 Discussion . . . . . . . . . . . . . . . . 61

5.5.1 BMI and prevalence of overweight and obesity . . . . . . 61

5.5 .2 Muscular strength . . . . . . . . . . . . . . . 62

5.5.3 Muscular strength and BMI . . . . . . . . . . . 63

5.5 .4 Limitations . . . . . . . . . . . . . . . . 63

5.6 Conclusion . . . . . . . . . . . . . . . . . 64

5.7 Summary: Study $2 \ldots \ldots \ldots$. . . . . . . . . . . . . 64

$\begin{array}{ll}\text { Thesis Map } & 67\end{array}$

6 Study $3 \quad 69$

6.1 A Cross-Sectional Study on the Socio-economic and Sex Differences in Health-Related Fitness Measures in School Children . . . . . . . . 69

6.2 Introduction . . . . . . . . . . . . . . . . . . 69

6.3 Methods . . . . . . . . . . . . . . . . . . 71

6.3.1 Participants and Settings . . . . . . . . . . . . 71

6.3.2 Measures and Procedures . . . . . . . . . . . . . . 71

6.3 .3 Statistical Analyses . . . . . . . . . . . . . . . 72

6.4 Results . . . . . . . . . . . . . . . . . . . 73

6.5 Discussion . . . . . . . . . . . . . . . 77

6.5 .1 Limitations . . . . . . . . . . . . . 80

6.6 Conclusion . . . . . . . . . . . . . . . 80

6.7 Summary: Study $3 \ldots \ldots$. . . . . . . . . . . . . . 81

$\begin{array}{lr}\text { Thesis Map } & 83\end{array}$

7 Study $4 \quad 85$

7.1 A dynamic assessment of children's physical competence: The Dragon Challenge . . . . . . . . . . . . . . . . 85

7.2 Introduction . . . . . . . . . . . . . . . 86

7.3 Methods . . . . . . . . . . . . . . . . . . . 89

7.3.1 Phase 1. Development of the DC . . . . . . . . . . . . 90

7.3.2 Phase 2. Surveillance Data and Construct Validity . . . . . . 96

7.3.3 Phase 3. Concurrent Validity and Reliability . . . . . . . . . . 97 
7.3.4 Statistical Analyses . . . . . . . . . . . . . . . . 99

7.4 Results . . . . . . . . . . . . . . . . . . 100

7.4 .1 Construct Validity . . . . . . . . . . . . . 102

7.4 .2 Concurrent Validity . . . . . . . . . . . . . . . . 107

7.4 .3 Reliability . . . . . . . . . . . . . . 107

7.5 Discussion . . . . . . . . . . . . . . . . 109

7.5.1 Construct Validity . . . . . . . . . . . 110

7.5.2 Concurrent Validity . . . . . . . . . . . . . . 111

7.5.3 Reliability . . . . . . . . . . . . . . . . . 112

7.5 .4 Feasibility . . . . . . . . . . . . 113

7.5.5 Limitations and Future Directions . . . . . . . . . . . . . 114

7.6 Conclusion . . . . . . . . . . . . . . . 115

7.7 Summary: Study $4 \ldots \ldots 115$

$\begin{array}{lr}\text { Thesis Map } & 117\end{array}$

8 Study $5 \quad 119$

8.1 Youth Physical Competence Promotion Model: A Quantitative Investigation into Modifiable Factors . . . . . . . . . . . . . . . . . . . 119

8.2 Introduction . . . . . . . . . . . . . . . . . . . . . . 119

8.3 Participants and Methods . . . . . . . . . . . . . . 121

8.3.1 Anthropometrics and Health-Related Fitness Measures . . . . 121

8.3.2 Questionnaires ................. . 122

8.3.3 Dragon Challenge . . . . . . . . . . . . 123

8.3.4 Statistical Analyses . . . . . . . . . . . . . . . 123

8.4 Results . . . . . . . . . . . . . . . . . . . . . . . 124

8.5 Discussion . . . . . . . . . . . . . . . . . . . . 129

8.5.1 Strengths and Limitations _. . . . . . . . . . . . 131

8.6 Conclusion . . . . . . . . . . . . . . . . . 132

8.7 Summary: Study $5 \ldots \ldots 132$

$\begin{array}{ll}\text { Thesis Map } & 135\end{array}$

9 Thesis Synthesis 139

9.1 Physical activity behaviours and influences and health-related fitness . 139

9.2 Physical competence monitoring and promotion . . . . . . . . . . . 140

9.3 Strengths and limitations . . . . . . . . . . . . . . . . . . . 143

9.4 Future directions . . . . . . . . . . . . . . . . . . . . . . 144

9.5 Final comments and reflection . . . . . . . . . . . . . . 146 


\section{Appendices}

Appendix I: Surveillance Surveys on Physical Activity and Sedentary Behaviour of Children in Wales (Study 1) . . . . . . . . . . . . . . 181

Appendix II: Swan-Linx Standard Operating Procedure and Training Tool . 187

Appendix III: Child Health and Activity Tool: Online Questionnaire . . . . 209 Appendix IV: Standard Operating Procedure for Child Health and Activity

Tool . . . . . . . . . . . . . . . . . . . . . 227

Appendix V: Dragon Challenge Manual . . . . . . . . . . . . 237

Appendix VI: Dragon Challenge Video Resources . . . . . . . . . . . . . . 275

Appendix VII: Children's Perceived Benefits/Barriers to Exercise Questionnaire . . . . . . . . . . . . . . . . . . . . 277

Appendix VIII: Children's Self-perceptions of Adequacy in and Predilection for Physical Activity Questionnaire . . . . . . . . . . . . 281

Appendix IX: Additional Publications . . . . . . . . . . . . . . . 285

Appendix X: ISPAH 2016 Poster Presentation . . . . . . . . . . . . 287

Appendix XI: BASES Presentation Abstract . . . . . . . . . . . . . . 289

Appendix XII: Active Healthy Kids Wales 2016 Report Card Press Release 291 Appendix XIII: Ethical Approvals . . . . . . . . . . . . . . . . . . . . 297

Swan-Linx application for ethical approval . . . . . . . . . . . . 297

Swan-Linx and Dragon Challenge application for ethical approval . . 315

Validating the Dragon Challenge application for ethical approval . . . 333 


\section{Acknowledgements}

Firstly, I would like to acknowledge the children and schools across Wales who participated in the studies in this thesis. I would like to thank the numerous A-STEM post-graduates, academics and practitioners from across Wales, as well as Gower College students, young ambassadors and the Active Young People Team Swansea and Bridgend, who helped with the administration of assessments in this thesis. The studies would not have been feasible without their significant contributions.

I would like to express my deepest appreciation to my first supervisor, Professor Gareth Stratton, for his unending support throughout my $\mathrm{PhD}$. His enthusiasm, dedication and encouragement has made this work possible. He has provided endless opportunities outside of my $\mathrm{PhD}$ to help my personal development and growth and has always managed to get best out of me. He has been a constant and vast source of knowledge and advice, and I cannot thank him enough for the impact he has made on my academic and life journey. I am also thoroughly grateful to my second supervisor, Dr. Kelly Mackintosh, for the time she has spent sharing her expertise and experience with me and for always being available to support me at a minute's notice. I would also like to thank Dr. Lawrence Foweather for his excellent advice and support during different stages of my $\mathrm{PhD}$.

I would further like to express my love and gratitude to my parents, Paul and Merci, who have always inspired me to pursue new heights and be the best I can be. While they may be far away, they have never wavered in their encouragement and reassurance. I would not be the person I am today without their love, understanding and incomparable parental support. I am also thankful to my big sister, Kat, for always being there for me whenever I needed her and for always backing me in anything I do.

I would like to extend my appreciation to my parents-in-law, Stephen and Tina, and sister-in-law, Tobi, for their indirect support and words of encouragement. I would also like to pay tribute to the late, Grandad Stanley Chicken, who always shared his immense wisdom and advice. You are thoroughly missed every day.

Last, but by no means least, I would like to thank my beautiful, intelligent, wonderful wife, Megan Tyler, who had to put up with me on my worst days, yet always found a way to find the positives in any situation. I cannot express the amount of love and support you have given me, not only throughout my $\mathrm{PhD}$, but ever since we met, 
10 years ago. With us recently celebrating our first (paper) wedding anniversary, I dedicate this thesis to you. This work would not have been possible without having you by my side, and I cannot put into words how important your contribution was, as well as how important you are to me. 


\section{List of Tables}

1 Data sources used to inform each grade allocated to the 10 indicators of the Active Healthy Kids Wales Report Card . . . . . . . . . . . . . 40

2 Grades according to physical activity indicator in the Active Healthy Kids Wales 2016 Report Card on physical activity for children and youth ........................ 41

3 Anthropometric measures (mean $\pm S D$ ) for boys and girls in 2003 and $2013 \ldots \ldots \ldots \ldots$. . . . . . . . . . . . . . . . 59

4 Proportion of boys and girls in 2003 and 2013 classed as unhealthy weight and percentage difference between 2003 and 2013 . . . . . . 60

5 Descriptive statistics (mean \pm SD) for Study 3 participants . . . . . . 74

$6 \quad$ Mean $\pm S D$ BMI z-scores by WIMD quintile (deprivation) groups . . 75

$7 \quad$ Adjusted mean \pm SE grip strength (adjusted by BMI z-scores) by WIMD quintile (deprivation) groups . . . . . . . . . . . . . 76

8 Adjusted mean \pm SE 20m MSRT (adjusted for maturation) by WIMD quintile (deprivation) groups . . . . . . . . . . . . . . . 77

9 Description of Dragon Challenge protocol and tasks and types of skills utilised during each task . . . . . . . . . . . . . . . . . . 92

10 Dragon Challenge assessment criteria . . . . . . . . . . . . . 95

11 Age (mean $\pm S D)$ and sex (\%) of participants who took part in the Dragon Challenge in study phase 2 and 3 . . . . . . . . . . . . . . 101

12 Descriptive statistics (mean $\pm S D$ ) for Dragon Challenge (surveillance data) score categories . . . . . . . . . . . . . . . . . . 102

13 Proportion (\%) of children successfully demonstrating each Dragon Challenge criterion (surveillance/normative data) . . . . . . . . . . 103

14 Rotated Component Matrix from Principal Component Analysis on Dragon Challenge criteria score . . . . . . . . . . . . . . . 105

15 Inter-and intra-rater reliability results for Dragon Challenge scores and sub-category scores . . . . . . . . . . . . . . . . . . . . 108

16 Descriptive statistics (mean \pm SD) of Study 5 measured variables . . 125 


\section{List of Figures}

1 Conceptual schematic of the Youth Physical Activity Model (YPAP-M) (Welk, 1999) . . . . . . . . . . . . . . . . . . . . 14

2 Front cover of the Active Healthy Kids Wales 2016 Physical Activity Report Card . . . . . . . . . . . . . . . . . . 42

3 Mean BMI z-scores for boys and girls in 2003 and 2013, with 95\% confidence intervals. . . . . . . . . . . . . . . . . . 60

$4 \quad$ Mean grip strength for boys and girls in 2003 and 2013, with 95\% confidence intervals. . . . . . . . . . . . . . . . . . 61

5 Factor structure of the Dragon Challenge . . . . . . . . . . . . . 106

6 Confirmatory Factor Analysis of the measured variables into four hypothesised latent factors . . . . . . . . . . . . . . . . . 126

7 Hypothesised Structural Equation Model . . . . . . . . . . . . . 127

8 Final Structural Equation Model evaluating the relationships between enabling, reinforcing, predisposing and physical competence latent variables . . . . . . . . . . . . . . . . . . . . . 128 


\section{Abbreviations}

20m MSRT
$\beta$

AHK

ALPHA

ANCOVA

ANOVA

A-STEM

BMI

BMI z-scores

BOT-2

CAMSA

CHAT

CFA

CFI

CI

$\mathrm{cm}$

COSMIN

CRF

CS4L: PLAYfun

DC

DEXA

$\mathrm{F}$

FMS

GFI

HBSC

ICC

IFI

$\mathrm{kg}$

KMO

LSOA 20m Multistage Shuttle Run Test

Standardised regression weight

Active Healthy Kids

Assessing Levels of Physical Activity - health-related test battery

Analysis of covariance

Analysis of variance

Applied Sport Technology Exercise and Medicine

Body mass index; weight $(\mathrm{kg})$ divided by height $\left(\mathrm{m}^{2}\right)$

Measure of relative body mass adjusted for child age and sex with use of reference data

Bruininks-Oseretsky Test of Motor Proficiency, Second Edition

Canadian Agility Movement Skill Assessment

Child Health and Activity Tool

Confirmatory factor analysis

Comparative fit index

Confidence interval

Centimetre

Consensus-based Standards for the Selection of Health

Measurement

Cardiorespiratory fitness

Canadian Sport for Life: Physical Literacy Assessment for Youth

Dragon Challenge

Dual-energy x-ray absorptiometry

F-test statistic; ratio of two variances

Fundamental or simple movement skills

Goodness of fit index

Health Behaviour in School-aged Children

Intraclass correlation coefficients

Incremental fit index

Kilogram

Kaiser-Meyer-Olkin

Lower-Layer Super Output Areas 


\begin{tabular}{|c|c|}
\hline $\mathrm{m} / \mathrm{m}^{2}$ & Metre/ square metre \\
\hline $\min (s)$ & Minute(s) \\
\hline MABC-2 & Movement Assessment Battery for Children-2 \\
\hline MSRT & Multistage shuttle run test \\
\hline MVPA & Moderate to vigorous intensity physical activity \\
\hline $\mathrm{n}$ & Number \\
\hline NGO & Non-government organisation \\
\hline NICE & National Institute for Health and Care Excellence \\
\hline $\mathrm{p}$ & Level of significance \\
\hline PA & Physical activity \\
\hline PCA & Principal component analysis \\
\hline $\mathrm{PE}$ & Physical Education \\
\hline PL & Physical literacy \\
\hline $\mathrm{r}$ & Coefficient of correlation \\
\hline$r^{2}$ & $\begin{array}{l}\text { Coefficient of determination; statistical measure that } \\
\text { represents the proportion of the variance for a dependent } \\
\text { variable that is explained by an independent variable }\end{array}$ \\
\hline RMSEA & Root mean square error of approximation \\
\hline RWG & Research working group \\
\hline $\mathrm{s}$ & Second \\
\hline SD & Standard deviation \\
\hline SE & Standard error \\
\hline SEM & Structural equation modelling \\
\hline SSA & Stability Skills Assessment \\
\hline $\mathrm{t}$ & $\begin{array}{l}\text { t-Test statistic; measure of the size of the difference relative } \\
\text { to the variation in the sample data }\end{array}$ \\
\hline TGMD-2/3 & Test of Gross Motor Development-2/3 \\
\hline TLI & Tucker-Lewis index \\
\hline UK & United Kingdom \\
\hline UNCRC & United Nations Convention on the Rights of the Child \\
\hline $\mathrm{VO}_{2 \max }$ & Maximal oxygen consumption \\
\hline WIMD & Welsh index of multiple deprivation \\
\hline YPAP-M & Youth Physical Activity Promotion Model \\
\hline
\end{tabular}




\section{Introduction}

\subsection{Rationale and background}

Regular physical activity and fitness are widely accepted as effective preventative measures for a variety of health risks and non-communicable diseases across all subgroups (e.g. age, sex, ethnicity, and socio-economic status) (Blair, Cheng, \& Holder, 2001; Department of Health, 2011; Lee et al., 2012; World Health Organization, 2018a). Engaging in adequate levels of physical activity in childhood can improve childhood health status (Andersen, Riddoch, Kriemler, \& Hills, 2011; Biddle \& Asare, 2011; Boreham \& McKay, 2011; Janssen \& Leblanc, 2010), result in improved adult health status (Lee et al., 2012; Warburton, Charlesworth, Ivey, Nettlefold, \& Bredin, 2010), and enable the formation of healthy behaviours that may be carried into adulthood (Boreham \& Riddoch, 2001; Kelly et al., 2007; Telama, 2009; Telama et al., 2014). For children and young people aged 5-18 years to receive the associated benefits of physical activity, the UK physical activity guidelines (Department of Health, 2011), recommend engagement in moderate to vigorous intensity activity for at least 60 minutes and up to several hours each day, and vigorous intensity activities at least three days per week. In Wales, the problem remains that activity levels are low and sedentary (sitting) behaviours are high and, in fact, the population of Wales is the least active in the UK (Department of Health, 2011). Therefore, promoting physical activity and the benefits on the quality and quantity of life is needed and requires a concerted effort.

Physical activity is multi-dimensional, complex behaviour, characterised by its frequency, intensity, and duration (Department of Health, 2011; World Health Organization, 2018b). There are different types of activity that contribute to overall physical activity and these occur in a multitude of settings, for example; occupational (e.g. work-related, school-related/Physical Education), household (e.g. domestic cleaning, caregiving, chores), transport (e.g. cycling/walking to school or work), leisure-time activities (e.g. dancing, active play), and organised sport participation (Department of Health, 2011; World Health Organization, 2018a). Moreover, there are numerous potential influencing factors on physical activity behaviours, highlighted in the Youth Physical Activity Promotion Model (YPAP-M) (Welk, 1999), such as those that may enable (e.g., movement skills/physical competence, health-related fitness, environmental attributes and access), predispose (e.g., perceived competence and selfefficacy) or reinforce (e.g., parental physical activity and family, peer and coach influence) physical activity in children and young people, as well as, demographic differ- 
ences (e.g. age, sex, ethnicity and socio-economic status). Physical activity is therefore notably difficult to assess (Dugdill \& Stratton, 2009). Few studies have succeeded in capturing temporal data on both the quantity and quality of physical activity across the different domains of physical activity. The 2012 and 2016 Lancet series on Physical Activity (Bauman et al., 2012; Hallal et al., 2012; Kohl et al., 2012; Sallis et al., 2016), advocated that "optimal physical activity monitoring and surveillance should focus on physical activity levels and behaviours, their determinants and outcomes, and indicators of proven and promising solutions to address low physical activity in various segments of the population." Specifically, it is suggested that measuring overall physical activity is not adequate for policy and intervention development (Bauman et al., 2012; Hallal et al., 2012; Kohl et al., 2012; Sallis et al., 2016). Instead, physical activity levels and behaviours, their determinants and outcomes should be the focus, and trends in physical activity across multiple domains should be monitored at the individual, social, environmental, and societal levels (Bauman et al., 2012; Hallal et al., 2012; Kohl et al., 2012; Sallis et al., 2016). With this objective, the Active Healthy Kids Global Alliance country report card series on physical activity, uses national surveillance data under key indicators to analyse physical activity behaviours, settings, and influences in children and young people (Tremblay et al., 2014).

Given the complexity of physical activity and its influencing factors (Bauman et al., 2012; Welk, 1999) and following the global recommendations from the Lancet series on physical activity (Bauman et al., 2012; Hallal et al., 2012; Kohl et al., 2012; Sallis et al., 2016), surveillance on overall physical activity levels as well as its subcomponents and determinants (e.g., active play/active leisure, organized sport, active transportation, sedentary time, physical fitness, physical literacy, physical competence), across subgroups (e.g. age, sex, ethnicity, and socio-economic status) is needed. Furthermore, a thorough and critical evaluation is needed for all sectors and settings that have a known impact on physical activity levels, including school, community and policy. Additionally, trends in physical activity behaviours, their determinants and outcomes across multiple domains should be examined. While the Active Healthy Kids Wales 2014 report card (Stratton et al., 2014), the inaugural report card to assess the 'State of the Nation' in relation to the levels of physical activity behaviours, settings, and influences in children and young people, began to address these aims, the continuation of the national report card and the monitoring of physical activity behaviours and influences on physical activity is needed.

Despite the importance of monitoring physical activity behaviours and influences in 
children and young people, it is postulated that assessing products or outcomes of physical activity could also give an indication of the quantity and quality of physical activity (Guinhouya, 2012), and could potentially present a more efficient marker of physical activity levels. Physical activity and physical fitness are closely associated, with physical fitness mainly, but not completely, determined by physical activity levels (Blair et al., 2001). Physical fitness includes a range components that are associated with health outcomes, such as cardiorespiratory fitness, muscular strength and body composition (Ruiz et al., 2011). In children, many studies have reported the strong associations between low cardiorespiratory fitness (McMurray \& Andersen, 2010; Ortega, Ruiz, Castillo, \& Sjöström, 2008; Ruiz, Castro-Piñero, et al., 2009), low muscular strength/ fitness (Ortega et al., 2008; Ruiz, Castro-Piñero, et al., 2009; Volaklis, Halle, \& Meisinger, 2015) and high body mass index (Griffiths, Parsons, \& Hill, 2010; L'Allemand-Jander, 2010; Ruiz, Castro-Piñero, et al., 2009), with metabolic and cardiovascular disease risk, mental health problems, reduced quality of life and all-cause mortality (Högström, Nordström, \& Nordström, 2015; Ruiz, Castro-Piñero, et al., 2009; Volaklis et al., 2015). Therefore, physical fitness is considered one of the most powerful indicators of health, both, in childhood and in adulthood (Ortega et al., 2008; Ruiz, Castro-Piñero, et al., 2009; Ruiz et al., 2011; Tomkinson, Carver, et al., 2017), independent of physical activity (Blaes, Baquet, Fabre, Van Praagh, \& Berthoin, 2011; Ekelund et al., 2007). In fact, physical fitness has been shown to be a stronger predictor of health than physical activity (Andersen et al., 2011). In addition to the associations with health, cardiorespiratory fitness, muscular strength and high body mass index are also associated with the acquisition of skills essential to physical activity and sporting success (Armstrong, Tomkinson, \& Ekelund, 2011; Ceschia et al., 2016). Given that measuring health-related fitness levels (i.e., components such as cardiorespiratory fitness, muscular strength and body mass index) not only provides a reflection of physical activity levels, but also supplies a predictor of current and future health, as well as, highlights the physical development of children and young people, there is an abundance of evidence highlighting the need to include health-related fitness testing as part of existing health and fitness screening and surveillance systems (Ortega et al., 2008; Ruiz, Castro-Piñero, et al., 2009; Ruiz et al., 2011; Tomkinson, Carver, et al., 2017). Nonetheless, there is currently no trend data on selected healthrelated fitness measures of school children in Wales, as well as, a scarcity of data on the levels of health-related fitness across sex and socio-economic status subgroups. Identifying changes and differences in health-related fitness levels among school children could indicate the need for services and interventions to improve the health and fitness inequalities in school-aged children. 
Apart from the physical fitness as an outcome measure of children and young people's physical activity levels, the development of motor skills and physical competence should be viewed as an additional and independent physiological factor related to physical activity that requires specific analyses (Guinhouya, 2012). Physical competence can be defined as "the sufficiency in movement vocabulary, movement capacities and developed movement patterns plus the deployment of these in a range of movement forms" (Whitehead, 2010). Overall, there is a vast array of evidence identifying physical competence as an important component of physical literacy, and, as such, a critical precursor for increasing positive health trajectories, particularly physical activity, across the lifespan (Barnett, Lai, et al., 2016; Edwards, Bryant, Keegan, Morgan, \& Jones, 2016; Hulteen, Morgan, Barnett, Stodden, \& Lubans, 2018; Robinson et al., 2015; Stodden et al., 2008; Whitehead, 2010). Specifically, systematic reviews and longitudinal studies have reported strong evidence for positive associations between physical competence and physical activity levels (Barnett, van Beurden, Morgan, Brooks, \& Beard, 2009; Holfelder \& Schott, 2014; Lopes, Rodrigues, Maia, \& Malina, 2011; Lubans, Morgan, Cliff, Barnett, \& Okely, 2010), health-related fitness (Barnett, van Beurden, Morgan, Brooks, \& Beard, 2008; Cattuzzo et al., 2016; Robinson et al., 2015) and perceived competence (Lubans et al., 2010; Robinson et al., 2015), as well as an inverse association with weight status (D'Hondt et al., 2014; Lubans et al., 2010; Robinson et al., 2015), in paediatric populations. Furthermore, studies have shown that enhanced physical competence during childhood tracks across the lifespan by leading to higher levels of physical activity and health-related fitness during adolescence (Barnett et al., 2009; Barnett, van Beurden, et al., 2008; Lopes et al., 2011; Robinson et al., 2015), and by supporting functional independence, general health and quality of life in later life, as well as reducing the risk of all-cause mortality (Hulteen et al., 2018; Paterson \& Warburton, 2010; Sigmundsson, Lorâs, \& Haga, 2016). Thus, enhanced physical competence in children and young people is foundational for physical activity promotion and associated health benefits, with transferable value throughout the life course.

There is a need to develop valid tools that effectively and efficiently assess physical competence in order to evaluate whether programmes are successful (Corbin, 2016). Physical competence includes not only the acquisition of health- and skill-related components of fitness (e.g. cardiorespiratory fitness, strength, flexibility, agility, balance, coordination, power, reactions and speed), but also movement skills, and plays an important role in the growth and development of children (Barnett, Lai, et al., 2016; 
Clark \& Metcalfe, 2002; Gallahue, Ozmun, \& Goodway, 2012; Hulteen et al., 2018; Robinson et al., 2015; Stodden et al., 2008; Whitehead, 2010). Movement skills consist of three interrelated constructs: fundamental movement skills (FMS; balance, core stability, coordination, speed variation, flexibility, control, proprioception, and power), combined movement (poise, fluency, precision, dexterity, and equilibrium), and complex movement (bilateral coordination, inter-limb coordination, handeye coordination, turning, twisting and rhythmic movements, and control of acceleration/deceleration) (Clark \& Metcalfe, 2002; Gallahue et al., 2012; Whitehead, 2010). The development of combined and complex movement skills is speculated to be imperative to increasing levels of physical competence in children over 8 years old (Clark \& Metcalfe, 2002; Gallahue et al., 2012; Tyler, Foweather, Mackintosh, \& Stratton, 2018; Whitehead, 2010), however most existing land-based movement skill assessments involve the performance of discrete skills in isolation (e.g., the Test of Gross Motor Development (TGMD-2/3) (Ulrich, 2000), the Bruininks-Oseretsky Test of Motor Proficiency, Second Edition (BOT-2) (Bruininks \& Bruininks, 2005), the Movement Assessment Battery for Children-2 (MABC-2) (Henderson, Sugden, \& Barnett, 2007), CS4L: Physical Literacy Assessment for Youth Fun (PLAYfun) (Cairney et al., 2018), Passport for Life: Movement Skills Assessment (Physical and Health Education Canada (PHE Canada), 2014)). Assessing discrete movement skills in isolation fails to account for the utilisation of combined and complex movement skills observed during physical activity and play and needed to demonstrate physical competence (Whitehead, 2010). In order to better measure elements of physical competence, there is a need to develop a dynamic assessment that requires the demonstration of fundamental (e.g., balance), combined (e.g., poise) and complex (e.g., rhythmic movements) movement skills through refined (complex) and specific movement patterns (e.g., hopping, turning, jumping patterns), and measured by both product/outcome- and process/technique-based evaluations. The assessment should also be feasible for national surveillance and could be used as both a formative and summative assessment in the educational context.

Ecological models provide a framework of potential influencing factors on healthrelated behaviours and are useful in emphasising social and psychological influences and environmental contexts (Barnett, Lai, et al., 2016; Bauman et al., 2012; Welk, 1999). As previously alluded to, research has investigated the mediating variable framework of the YPAP-M (Chen, Welk, \& Joens-Matre, 2014), however the examination of the influencing factors on physical competence, guided by this model, remains to be explored. Further, few studies have investigated both psychological 
influences and environmental factors on physical competence (Barnett, Lai, et al., 2016). Therefore, there is a need to quantify the direct and indirect relationships between modifiable factors on physical competence and to investigate potential sex, weight status, and school level differences. The development of an ecological model with physical competence as the endogenous variable would afford new insight and an in-depth understanding of the multiple influences on physical competence. Such a targeted approach could inform intervention development with the objective to promote physical competence in children, as well as explain effects or lack of effects in current intervention strategies.

\subsection{Problem statement}

Promoting physical activity, fitness and physical literacy are effective preventative measures for a variety of health risks and non-communicable diseases. Moreover, identifying levels of physical activity, health-related fitness and motor competence among school children is needed to inform effective services and interventions. The current challenge is to identify the interrelationships between these factors and to develop new insight into how these factors can be assessed and integrated into physical activity frameworks and models.

\subsection{Thesis aims}

The overarching aim of the present thesis was to investigate the health, fitness, lifestyle and physical competency of school children in Wales. This $\mathrm{PhD}$ Thesis is divided into five study chapters:

Study 1: The purpose of Study 1 was to consolidate and translate research related to physical activity among children and youth in Wales and to synthesise national surveillance data from several surveys in order to assign grades to physical activity/inactivity behaviours and influences indicators, as well as to examine trends or inequalities in the data.

Study 2: The aim of Study 2 was to highlight the secular changes in selected health and fitness measures (body mass index, prevalence of overweight and obesity, and grip strength) in 10-11-year-old school children, using data obtained in 2003 and 2013. 
Study 3: The aim of Study 3 was to investigate socio-economic and sex differences in selected health-related fitness measures in 9-12-year-old children.

Study 4: The first aim of Study 4 was to develop a land-based dynamic measure of physical competence that requires a participant to demonstrate fundamental, combined and complex movement skills, movement capacities/skills and movement patterns, and assessors to score both processes and products (Dragon Challenge; DC). The second aim was to assess the psychometric properties of the DC in 10-14-yearold children.

Study 5: The aim of Study 5 was first to quantify the direct and indirect relationships between enabling, predisposing and reinforcing socio-ecological factors on physical competence, and second to investigate potential sex, weight status, and school level differences. 


\section{Literature Review}

Physical activity enhances health benefits in children (Biddle \& Asare, 2011; Boreham \& McKay, 2011; Janssen \& Leblanc, 2010), while preventing emerging health risk factors (Andersen et al., 2011; Janssen \& Leblanc, 2010). There are strong associations between low physical fitness (McMurray \& Andersen, 2010; Ortega et al., 2008; Ruiz, Castro-Piñero, et al., 2009; Volaklis et al., 2015) and high body mass index (Griffiths et al., 2010; L'Allemand-Jander, 2010; Ruiz, Castro-Piñero, et al., 2009), as well as with disease risk, mental health problems, reduced quality of life and all-cause mortality later in life (Högström et al., 2015; Ruiz, Castro-Piñero, et al., 2009; Volaklis et al., 2015). Physical competence is a critical precursor for increasing positive health trajectories, particularly physical activity and physical fitness, across the lifespan (Barnett, Lai, et al., 2016; Edwards et al., 2016; Hulteen et al., 2018; Robinson et al., 2015; Stodden et al., 2008; Whitehead, 2010). Adequate levels of physical activity, physical fitness and physical competence in childhood can improve childhood health status, result in improved adult health status (Högström et al., 2015; Lee et al., 2012; Robinson et al., 2015; Ruiz, Castro-Piñero, et al., 2009; Stodden et al., 2008; Volaklis et al., 2015; Warburton et al., 2010; Whitehead, 2010), and enable the formation of healthy behaviours that may be carried into adulthood (Boreham \& Riddoch, 2001; Kelly et al., 2007; Telama, 2009; Telama et al., 2014). With this in mind, this literature review aims to delve deeper into the evidence base surrounding physical activity, physical fitness and physical competence, and the monitoring and surveillance of such variables.

\subsection{Physical Activity}

Physical activity is defined as "any bodily movement produced by skeletal muscles that require energy expenditure" (World Health Organization, 2018b). Physical activity includes all forms of activity, such as active travel (e.g., walking and cycling), organised and competitive sport, active recreation (e.g., dance, yoga), playing active games, as well as work-related activity (Department of Health, 2011; World Health Organization, 2018a). Physical activity a key determinant of energy expenditure, and is fundamental to energy management (Besson et al., 2009; Chaput et al., 2011; Hills, Andersen, \& Byrne, 2011; Monasta et al., 2010; Warburton et al., 2010). Engaging in sufficient physical activity is widely accepted as an effective preventative measure for a variety of health risks across all subgroups (e.g. age, sex, ethnic, and socioeconomic) (Department of Health, 2011; World Health Organization, 2018a). Physical activity decreases the risk of many non-communicable diseases (Besson et al., 
2009; Chaput et al., 2011; Dziura, Kasl, \& Di Pietro, 2004; Hills et al., 2011; Lee et al., 2012; Li \& Siegrist, 2012; Miller \& Dunstan, 2004; Monasta et al., 2010; Paterson \& Warburton, 2010; Sattelmair et al., 2011; Stuck et al., 1999; Taylor et al., 2004; Warburton et al., 2010; Wolin, Yan, \& Colditz, 2011). In particular, regular and sufficient levels of physical activity in adults reduces the risk of hypertension (Warburton et al., 2010), coronary heart disease (Lee et al., 2012; Sattelmair et al., 2011; Warburton et al., 2010), cardiovascular disease (Li \& Siegrist, 2012; Warburton et al., 2010), stroke (Warburton et al., 2010), diabetes (Dziura et al., 2004; Lee et al., 2012; Miller \& Dunstan, 2004), and breast, colon and endometrial cancer (Warburton et al., 2010; Wolin et al., 2011). Physical activity also reduces depression and promotes well-being (Das \& Horton, 2012; Mammen \& Faulkner, 2013; Schuch et al., 2016), reduces the risk of falls, and improves bone and functional health (Paterson \& Warburton, 2010; Stuck et al., 1999; Taylor et al., 2004). These reductions in risk range from $20 \%$ to 83\% (Besson et al., 2009; Chaput et al., 2011; Dziura et al., 2004; Hills et al., 2011; Lee et al., 2012; Li \& Siegrist, 2012; Miller \& Dunstan, 2004; Monasta et al., 2010; Paterson \& Warburton, 2010; Sattelmair et al., 2011; Stuck et al., 1999; Taylor et al., 2004; Warburton et al., 2010; Wolin et al., 2011). Moreover, sufficient levels of physical activity can lower the risk of early death by $30 \%$, and therefore extends life expectancy (Lee et al., 2012). Physical inactivity (lack of sufficient physical activity) has been identified as the fourth leading risk factor for global mortality, and is estimated to cause $16.9 \%$ of all deaths in the UK (Lee et al., 2012). Furthermore, the cost of physical inactivity has been estimated as $1.5-3.0 \%$ of total direct health care costs in the UK, equivalent to $£ 0.9$ billion (Oldridge, 2008). Reducing physical inactivity by just one percent a year over a five-year period would save the UK economy just under $£ 1.2$ billion (UKActive, 2014). Despite these health and economic benefits, in Wales, the problem remains that activity levels are low and sedentary (sitting) behaviours are high and, in fact, the population of Wales is the least active in the UK (Department of Health, 2011). Therefore, promoting the benefits of physical activity on the quality and quantity of life of people represents an excellent, cost effective investment (UKActive, 2014) and requires a concerted effort.

\subsection{UK Physical Activity Guidelines}

The UK physical activity guidelines 'Start Active Stay Active' (Department of Health, 2011), were published on behalf of the Chief Medial Officers of the four home countries in July 2011. These guidelines recommend levels of physical activity for health in children under 5 years (early years), children and young people aged 5 to 18 years and adults and seniors (older adults). 


\subsection{Physical Activity and Sedentary Behaviours in Children and Young People}

Adequate levels of physical activity in childhood can improve childhood health status, result in improved adult health status, and enable the formation of healthy behaviours that may be carried into adulthood (Boreham \& Riddoch, 2001; Kelly et al., 2007; Telama, 2009; Telama et al., 2014). There is a strong body of evidence, consisting of both observational and experimental research, that indicates that regular physical activity provides short and long-term health benefits (Department of Health, 2011). Specifically, physical activity is associated with numerous physiological, psychosocial and cognitive benefits (Biddle \& Asare, 2011; Boreham \& McKay, 2011; Janssen \& Leblanc, 2010), and prevents emerging health risk factors in both healthy and high risk children and young people (Andersen et al., 2011; Janssen \& Leblanc, 2010). Moreover, physical activity has wider social benefits and promotes well-being and quality of life across the life course. The literature base continues to grow, with large-scale studies providing evidence of the strong associations that clarify the dose-response relationships between physical activity and health outcomes in children and young people (Andersen et al., 2006; Artero, Ruiz, et al., 2011; Department of Health, 2011; Dumith, Gigante, Domingues, \& Kohl, 2011; Ekelund et al., 2007, 2006; Janssen \& Leblanc, 2010). Alternatively, sedentary behaviours (any behaviours that occur whilst sitting or lying down while awake and typically require very low energy expenditure (Pate, O’Neill, \& Lobelo, 2008) are related to poor health outcomes, such as cardio-metabolic diseases, poorer mental health, and all-cause mortality, regardless of an individual's physical activity levels (Biddle \& Asare, 2011; Chinapaw, Proper, Brug, van Mechelen, \& Singh, 2011; Marshall, Biddle, Gorely, Cameron, \& Murdey, 2004; Tremblay, Colley, Saunders, Healy, \& Owen, 2010; Tremblay et al., 2011). Therefore, it is important to identify childhood physical activity levels and sedentary behaviours to indicate current, and future, health.

For children and young people aged 5-18 years to receive the associated benefits of physical activity, the UK physical activity guidelines (Department of Health, 2011), recommend engagement in moderate to vigorous intensity activity for at least 60 minutes and up to several hours each day, and vigorous intensity activities at least three days per week. Further, all children and young people should minimise the amount of time in sedentary (sitting) behaviours for long periods of time. The British Heart Foundation summarised international recommendations from North America, Australia and the United Kingdom (British Heart Foundation National Centre (BHFNC) for Physical Activity and Health Loughborough University, 2012), and provided the 
recommendation that children and young people should spend less than 2 hours per day in sedentary behaviours (British Heart Foundation National Centre (BHFNC) for Physical Activity and Health Loughborough University, 2012). More recently, a special supplement of Applied Physiology, Nutrition, and Metabolism ("CSEP Special Supplement - Canadian 24-Hour Movement Guidelines for Children and Youth: An Integration of Physical Activity, Sedentary Behaviour, and Sleep," 2016), included a collection of the background research, consultations, processes, and outcomes that led to the development of the Canadian 24-Hour Movement Guidelines for Children and Youth (aged 5-17 years), the first 24 hour guidelines that integrated physical activity, sedentary behaviour, and sleep (Tremblay, Carson, \& Chaput, 2016; Tremblay, Carson, Chaput, et al., 2016). The supplement also contained four systematic reviews, which assessed findings from nearly 600 published articles, and provided evidence for the development of the guidelines (Tremblay, Carson, \& Chaput, 2016; Tremblay, Carson, Chaput, et al., 2016). These reviews examined the associations of physical activity (total physical activity, MVPA, and light physical activity) (Poitras et al., 2016), sedentary behaviours (Carson et al., 2016), sleep duration (Chaput et al., 2016), and combinations of objectively measured physical activity and subjectively measured sedentary behaviour and sleep (Saunders et al., 2016), with health outcomes in school-aged children and youth. Taken together these reviews suggest that for children and youth (aged 5-17 years) to obtain optimal health benefits, they should achieve high levels of physical activity, low levels of sedentary behaviour, and sufficient sleep (Carson et al., 2016; Chaput et al., 2016; Poitras et al., 2016; Saunders et al., 2016; Tremblay, Carson, Chaput, et al., 2016). Specifically, to achieve a healthy 24 hours, children and youth should; i) achieve 9 to 11 hours of uninterrupted sleep per night for 5-13 year olds and 8 to 10 hours for 14-17 year olds, ii) accumulate at least 60 minutes of moderate to vigorous physical activity per day, involving a variety of aerobic activities, and vigorous physical activity and muscle and bone strengthening activities on at least 3 days per week, iii) achieve several hours of a variety of structures and unstructured light physical activity, iv) spend no more than 2 hours per day in recreational screen time, and limit sitting for extended periods (Carson et al., 2016; Chaput et al., 2016; Poitras et al., 2016; Saunders et al., 2016; Tremblay, Carson, Chaput, et al., 2016)

\subsection{Ecological Models}

To develop successful targeted interventions, an appropriate conceptual promotion model should be utilised to prioritise the key characteristics of the target group and to produce a comprehensive and structured assessment of needs and barriers to a healthy 
behaviour or outcome through a range of social, behavioural, physical and environmental factors (Gielen, McDonald, Gary, \& Bone, 2008; Green, Kreuter, Deeds, \& Partridge, 1980; Welk, 1999). The PRECEDE-PROCEED model (Green et al., 1980) is a health promotion planning model, that provides a structured organisational framework or road map for determining health education programmes for a variation of behaviours or outcomes (Gielen et al., 2008; Green et al., 1980; Welk, 1999). The PRECEDE-PROCEED model outlines a sequence of stages that should be conducted. The first stage is a behaviour and environmental assessment to identify the primary determinants of a given behaviour or outcome (Gielen et al., 2008; Green et al., 1980; Welk, 1999). The second stage is an educational and organisational assessment for categorising the determinants into factors that enable, predispose, or reinforce the given behaviour or outcome, and then weighting these on their potential influence (Gielen et al., 2008; Green et al., 1980; Welk, 1999). The final stage is administrative and policy assessment (Gielen et al., 2008; Green et al., 1980; Welk, 1999), including the assessment of resources, budgets, organisations, etc., that should be conducted to refine interventions or promotional strategies that are likely to be effective based on the ecological model produced in the other stages. Focused interventions should then be followed by process, impact and outcome evaluations to measure the effectiveness of the intervention (Gielen et al., 2008; Green et al., 1980; Welk, 1999).

The Youth Physical Activity Promotion Model (YPAP-M; Figure 1) (Welk, 1999), utilises aspects of the PRECEDE-PROCEED model, to offer an ecological conceptual model framing factors that may predispose, reinforce or enable physical activity in children, as well as, personal demographic factors (Welk, 1999). 


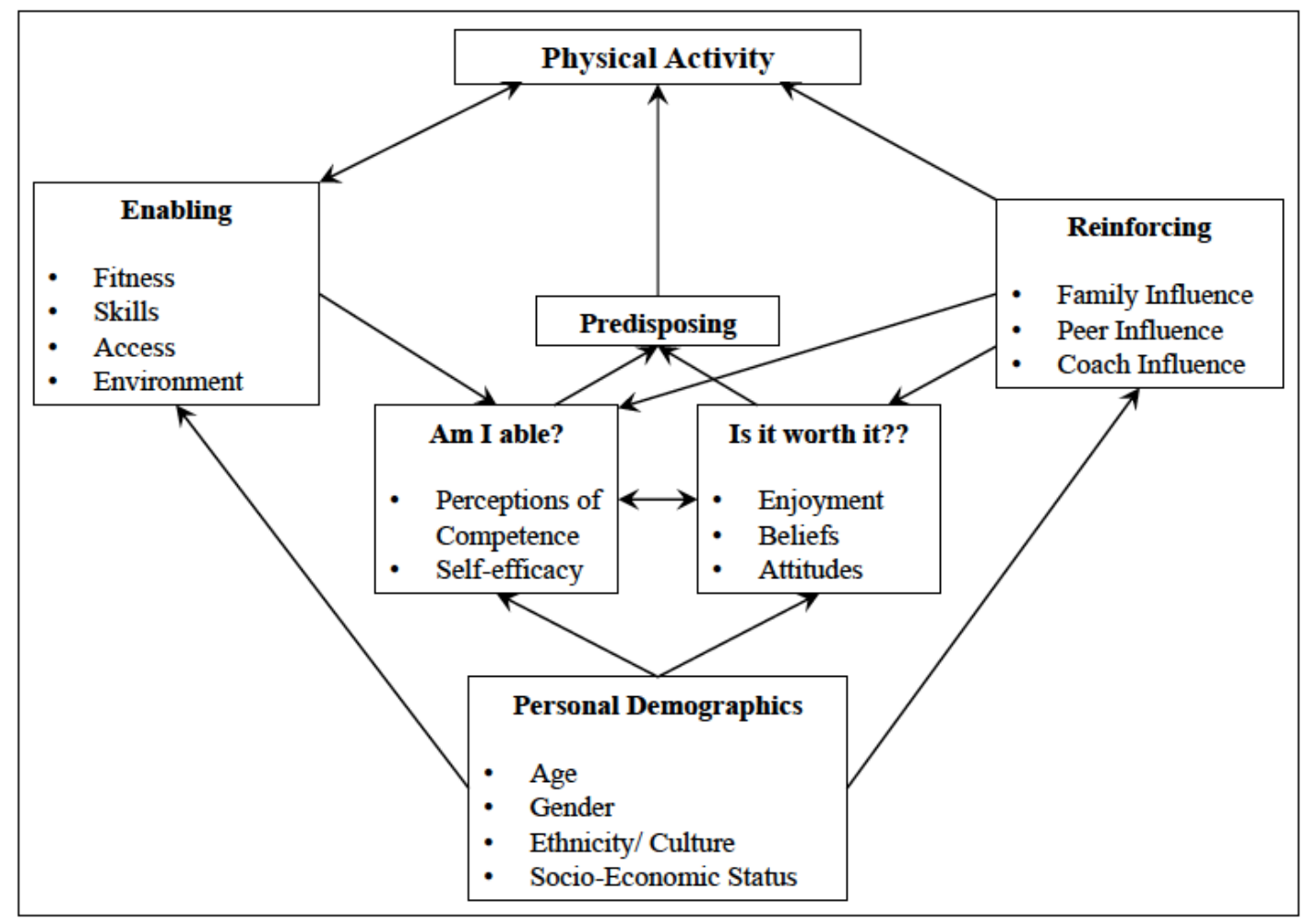

Figure 1. Conceptual schematic of the Youth Physical Activity Model (YPAP-M) (Welk, 1999)

Predisposing factors include variables that collectively increase the likelihood that a child will be physically active on a regular basis (Welk, 1999). These factors are based on self-efficacy (Bandura, 1986), perceived competence (Deci \& Ryan, 1985; Harter, 1978) perceived behavioural control (Ajzen, 1985) and facilitating conditions (Triandis, 1977), and are reduced to two fundamental questions: Is it worth it? And Am I able to? (Welk, 1999). The first question refers to benefits and costs of physical activity and includes cognitive and affective components, whilst the second refers to perceived competence (Welk, 1999). Reinforcing factors include variables that reinforce children's physical activity behaviours, and include parents/guardians facilitating and encouraging their child to be physically active (Welk, 1999). Enabling factors include variables that allow children to be physically active, and consist of biological/physiological factors such as movement skills/physical competence, health-related fitness, as well as, environmental attributes and access factors (Welk, 1999). Demographic factors are the individual differences that have been shown to have an influence on physical activity (e.g. age, sex, ethnicity and socio-economic status) (Welk, 1999). Overall, the YPAP-M provides an integrated framework for understanding the factors that influence physical activity. 
Research has shown that there are numerous applications of ecological models (Glanz, Rimer, \& Viswanath, 2008). These models provide a valid theoretical or causal framework of potential influencing factors on health-related behaviours or outcomes and are useful in emphasising social and psychological influences and environmental contexts (Glanz et al., 2008). As such, the PRECEDE-PROCEED and the YPAP-M approaches can be used as a guide to characterise various influences on any behaviour or outcome related to children and young peoples' health, growth and development. The construction of an ecological model/framework using these approaches can then lead to future interventions and programmes targeted to the specific behaviour or outcome.

Aspects of the PRECEDE-PROCESS model and YPAP-M are utilised in Study 1 to produce a comprehensive and structured assessment of needs and barriers to a healthy behaviour (physical activity behaviours vs. sedentary behaviours) or outcomes (physical fitness, physical competence) through a range of social, behavioural, physical and environmental factors. The YPAP-M was also considered and altered in Study 5 to produce a framework to improve the physical competency levels in children, and to quantify the relationships between socio-ecological factors and physical competence to inform future intervention strategies.

\subsection{Monitoring Physical Activity}

Physical activity is multi-dimensional, complex behaviour, characterised by its frequency, intensity, and duration (Department of Health, 2011; World Health Organization, 2018b). There are different types of activity that contribute to overall physical activity and these occur in a multitude of settings, for example; occupational (e.g. work-related, school-related/PE), household (e.g. domestic cleaning, caregiving, chores), transport (e.g. cycling/walking to school or work), leisure-time activities (e.g. dancing, active play), and organised sport participation (Department of Health, 2011; World Health Organization, 2018a). Physical activity (and its alternate sedentary behaviour) is therefore notably difficult to assess (Dugdill \& Stratton, 2009). Most research has attempted to quantify physical activity, although there is no single method that captures the complete range of physical activity characteristics. Few studies have succeeded in capturing temporal data on both the quantity and quality of physical activity.

The gold standard measures of physical activity are those that acquire objective data (Hallal et al., 2012; Sherar et al., 2011). Objective data can be obtained by use of accelerometers, pedometers, heart-rate telemeters or electronic motion sensors (dou- 
bly labelled water, actiheart) (Hallal et al., 2012; Lubans, Smith, Harries, Barnett, \& Faigenbaum, 2014; Sherar et al., 2011). Although objective measures are the desired measures of physical activity, achieving a population sample with any of these measures can be extremely costly and very time consuming for both participants and staff. Also, most objective measures do not distinguish between different domains of physical activity (e.g., active transport, sport participation). Therefore, the 2012 and 2016 Lancet series on Physical Activity (Hallal et al., 2012; Kohl et al., 2012; Sallis et al., 2016), advocated that "optimal physical activity monitoring and surveillance should focus on physical activity levels and behaviours, their determinants and outcomes, and indicators of proven and promising solutions to address low physical activity in various segments of the population". Specifically, it is suggested that measuring overall physical activity is not adequate for policy and intervention development (Hallal et al., 2012; Kohl et al., 2012; Sallis et al., 2016). Instead, trends in physical activity across multiple domains should be monitored at the individual, social, environmental, and societal levels (Hallal et al., 2012; Kohl et al., 2012; Sallis et al., 2016). The monitoring of levels and surveillance of trends in physical activity behaviours and influences on physical activity were incorporated in Study 1, which sought to consolidate and translate research related to physical activity among children and young people in Wales.

\subsection{Product/Outcome Measure of Physical Activity - Physical Fit- ness}

Physical fitness is "the capacity to perform physical activity or physical exercise, and refers to a full range of physiological and psychological qualities" (Janssen \& Leblanc, 2010; Ortega et al., 2008; Ruiz, Castro-Piñero, et al., 2009; Ruiz et al., 2011). Physical activity and physical fitness are closely associated, with physical fitness mainly, but not completely, determined by physical activity levels (Blair et al., 2001). Genetic contributions to physical fitness are important, but account for less of the variation observed in physical fitness than is due to environmental factors, predominantly physical activity (Blair et al., 2001; Bouchard \& Pérusse, 1994). Therefore, physical activity is one of the main antecedents of physical fitness, and recent reviews have shown that regular moderate and vigorous levels of physical activity increased levels of physical fitness (Janssen \& Leblanc, 2010; Poitras et al., 2016).

Physical fitness is multidimensional and refers to two goals: performance, which consists of six skill-related fitness components, and health that includes five health-related 
fitness components, each of which contributes to total quality of life (Bouchard \& Shephard, 1994; Corbin, Welk, Corbin, \& Welk, 2006). It is understood that physical fitness is an integrated measure of all body functions and structures, including, skeletomuscular, cardiorespiratory, haematocirculatory, psychoneurological and endocrine-metabolic, that are involved in the performance of daily physical activity and/or physical exercise (Ortega et al., 2008; Ruiz, Castro-Piñero, et al., 2009; Ruiz et al., 2011). Therefore, when physical fitness is assessed, the functional status of all these systems are also being tested (Ortega et al., 2008). For this reason, physical fitness is now considered one of the most powerful markers of health, both, in childhood and in adulthood (Ortega et al., 2008; Ruiz, Castro-Piñero, et al., 2009; Ruiz et al., 2011; Tomkinson, Carver, et al., 2017), independent of physical activity (Blaes et al., 2011; Ekelund et al., 2007). In fact, physical fitness has been shown to be a stronger marker/predictor of health than physical activity (Andersen et al., 2011). Physical fitness tracks moderately well from childhood through to adulthood (Andersen, Hasselstrøm, Grønfeldt, Hansen, \& Karsten, 2004; Cleland, Ball, Magnussen, Dwyer, \& Venn, 2009; Kristensen et al., 2006; Ortega et al., 2008; Twisk, Kemper, \& Van Mechelen, 2009). Low levels of physical fitness in children and young people are significantly associated with increased risk of cardio-metabolic diseases (Dwyer et al., 2009; Ortega et al., 2008; Ortega, Silventoinen, Tynelius, \& Rasmussen, 2012) and obesity (Ortega et al., 2011), reduced quality of life (Morales et al., 2013) and poorer skeletal (Moliner-Urdiales et al., 2010) and mental health (Ruiz, Castro-Piñero, et al., 2009), later in life. In addition to health associations, these fitness measures are also associated with the acquisition of skills essential to physical activity and sporting success (Armstrong et al., 2011; Ceschia et al., 2016). Therefore, identifying differences in fitness levels among school children is important in the surveillance of health, as well as, for monitoring the growth and development of children.

Health-related fitness are components of physical fitness that are specifically associated with health outcomes, e.g. cardiorespiratory fitness, muscular fitness/strength, and body composition (Bouchard \& Shephard, 1994; Ruiz et al., 2011). These healthrelated components of fitness can be objectively and accurately measured using laboratory methods, although the application of these laboratory measures in a schoolbased setting is limited due to the need for sophisticated equipment, highly trained technicians and time constraints (Artero, Espaa-Romero, et al., 2011; Castro-Piñero et al., 2010; Ruiz, Castro-Piñero, et al., 2009). Field-based measures provide a reasonable alternative, as they are time- and cost-efficient, require minimal equipment and can be easily administered to many participants simultaneously, in a school based 
setting, and for population-based studies (Artero, Espaa-Romero, et al., 2011; CastroPiñero et al., 2010; Ruiz, Castro-Piñero, et al., 2009). Over the past two decades, there are numerous field-based test batteries that have been developed to assess healthrelated fitness in children and young people populations (Castro-Piñero et al., 2010). Three of the most well-known tests used in children and young people are: i) the FITNESSGRAM Test Battery in the USA (Plowman \& Meredith, 2013), ii) the EUROFIT Test Battery (Council of Europe, 1993), and iii) the Assessing Levels of Physical Activity (ALPHA) Health-related Fitness Test Battery in Europe (Ruiz et al., 2011), all of which provide a set of valid, reliable, feasible and safe field-based tests for the assessment of health-related fitness in children and young people (Castro-Piñero et al., 2010).

\subsubsection{Cardiorespiratory Fitness}

The most frequently evaluated health-related fitness component is cardiorespiratory fitness (CRF) (Tomkinson, Lang, Tremblay, et al., 2017). Cardiorespiratory fitness, also called cardiovascular fitness, cardiorespiratory endurance or maximal aerobic power, is the overall capacity of the cardiovascular and respiratory systems to transport oxygen to the skeletal muscles and to utilise it to generate energy to support muscle activity during prolonged strenuous dynamic exercise (Armstrong et al., 2011; Artero, Ruiz, et al., 2011; Castro-Piñero et al., 2010; Ruiz, Castro-Piñero, et al., 2009; Tomkinson, Lang, Tremblay, et al., 2017). In children and young people, CRF is a predictor of cardiovascular disease risk factors such as, abnormal blood lipids, high blood pressure and overall and central adiposity, as well as, metabolic syndrome and arterial stiffness, cancer and mental health, later in life (Ortega et al., 2008; Ruiz, Castro-Piñero, et al., 2009). Furthermore, there is evidence of an interaction between CRF and adiposity, suggesting that high levels of CRF may reduce the effects of being overweight or obese in children and young people (Eisenmann et al., 2005; Jakicic \& Davis, 2011; McAuley \& Blair, 2011). Cardiorespiratory fitness is a product of vigorous physical activity (Ortega et al., 2008), therefore measuring CRF provides insight into the levels of children's physical activity, as well as the capabilities of several bodily systems that are involved in the performance of physical activity and exercise (Ortega et al., 2008; Tomkinson, Lang, Tremblay, et al., 2017). In addition, CRF tracks from childhood through to adulthood moderately well (Malina, 2001; Ortega et al., 2013), showing that the measurement of CRF in children provides information into current and future health.

Maximal oxygen consumption $\left(\mathrm{VO}_{2 \max }\right)$ obtained through a graded maximal exercise 
to voluntary exhaustion test, is considered as the single best indicator of CRF (Shephard et al., 1968). The most common worldwide field-based assessment and estimate of CRF in children and young people is the $20 \mathrm{~m}$ multistage shuttle run test $(20 \mathrm{~m}$ MSRT) (Olds, Tomkinson, Léger, \& Cazorla, 2006; Tomkinson, Lang, Tremblay, et al., 2017; Tomkinson, Léger, Olds, \& Cazorla, 2003; Tomkinson \& Olds, 2007, 2008). This test has been shown to be an excellent population-based surveillance and monitoring tool as it is time-, cost- and labour-efficient through its ability to test multiple individuals simultaneously with minimal equipment and across different testing locations (indoors, outdoors, smaller spaces) (Melo et al., 2011; Ruiz, Silva, et al., 2009; Tomkinson \& Olds, 2008). Further, the 20m MSRT has been shown to display good validity and reliability (Tomkinson \& Olds, 2008), and $\mathrm{VO}_{2 \max }$ can be estimated from the score obtained in the 20m MSRT with use of certain equations (Léger, Mercier, Gadoury, \& Lambert, 1988).

Levels of cardiorespiratory fitness in children and young people, have declined globally as well as in the UK (England) in recent years (Boddy, Fairclough, Atkinson, \& Stratton, 2012; Sandercock, Voss, McConnell, \& Rayner, 2010; Stratton et al., 2007; Tomkinson, Lang, \& Tremblay, 2017; Tomkinson et al., 2003; Tomkinson \& Olds, 2007). Boddy et al. (2012), investigated changes in CRF from 1998 to 2010 using SportsLinx data on 9-11 year old children from England. Findings from the study identified a significant decline in CRF over time from 1998 to 2010, with all boys in cohorts 2000-2010 and girls in cohorts 2003-2010 having significantly lower CRF than baseline (1998), regardless of weight status (Boddy et al., 2012). The study also reported that the observed mean declines in CRF of $1.34 \%$ for boys and $2.29 \%$ in girls were greater than those reported globally (Boddy et al., 2012; Tomkinson et al., 2003). Tomkinson et al. (2017), examined global trends in CRF in children and young people aged 9-17 years from 19 high-income and upper middle-income countries between 1981 and 2014. The analysis indicated that since 1981 there was a considerable international decline of $-7.3 \%$ in CRF, but only negligible changes were apparent since 2000 (Tomkinson, Lang, \& Tremblay, 2017). This study also identified that although most countries displayed a decline in CRF (-3.6\% pre-2000, $-0.9 \%$ post-2000), the rate and pattern of change varied across countries, however the declines in CRF were consistently larger for boys than girls (boys: $-4.2 \%$ pre-2000, $-1.4 \%$ post-2000; girls: $-3.0 \%$ pre-2000; $-0.6 \%$, post-2000) (Tomkinson, Lang, \& Tremblay, 2017). As low CRF is significantly associated with key determinants of health (Ortega et al., 2008; Ruiz, Castro-Piñero, et al., 2009), these reported declines are suggestive of declines in population health. Therefore, continued monitoring and insight is needed to inform 
interventions, programmes and policies is encouraged.

\subsubsection{Muscular Strength}

Muscular strength refers to the ability of a specific muscle or muscle group to generate maximum force against a resistance in a single contraction (Bouchard \& Shephard, 1994; Corbin et al., 2006; Ortega et al., 2008; Ruiz et al., 2006). Muscular strength is an important aspect of fitness and health status, as well as, provides a marker of vigorous intensity physical activities, including those that strengthen muscle and bone. A decrease of muscular strength may cause significant functional limitations (Wind, Takken, Helders, \& Engelbert, 2010). In children and young people, increased muscular strength has been associated with reductions in total and central adiposity and cardiovascular risk factors, and increases in bone health, self-esteem and perceived competence (Smith et al., 2014). In adults, emerging evidence suggests that handgrip strength is a strong predictor of all-cause mortality, morbidity, and the expectancy of being able to live independently (García-Hermoso et al., 2018; Metter, Talbot, Schrager, \& Conwit, 2002). Consequently, more attention is now being given to assessing muscular strength in children, as a predictor of health outcomes and all-cause mortality (Cohen et al., 2011; De Miguel-Etayo et al., 2014; Moliner-Urdiales et al., 2010; Ruiz, Castro-Piñero, et al., 2009; Silverman, 2011; Tremblay, Shields, et al., 2010).

As the generation of maximum force is determined by several factors (e.g. the number and size of muscles, the proportion of muscles fibres, the coordination of the muscle groups), there is no single test for measuring overall muscle strength (Ortega et al., 2008). However, the handgrip strength test is one of the most used tests for assessing muscular strength in experimental and epidemiological studies (Cohen et al., 2011; De Miguel-Etayo et al., 2014; Moliner-Urdiales et al., 2010; Ruiz, Castro-Piñero, et al., 2009; Silverman, 2011; Tremblay, Shields, et al., 2010). Handgrip strength refers to the maximal isometric force that can be mainly generated by the hand and forehand muscles involved (Ruiz et al., 2006). The handgrip strength test is a simple and economical test that gives practical information on muscle, nerve, bone or joint disorders (Ortega et al., 2008; Ruiz et al., 2011, 2006). Moreover, the handgrip strength test is correlated with many other measures of strength and is therefore a good indicator of total muscular strength (Wind et al., 2010). Studies on grip strength as a measure of muscular strength have revealed its direct associations with chronic diseases, multi-morbidity and premature mortality (Cheung, Nguyen, Au, Tan, \& Kung, 2013; Metter et al., 2002; Ortega et al., 2008; Ruiz, Castro-Piñero, et al., 2009; Volaklis et 
al., 2015).

Akin to levels of CRF, levels of muscular strength in children and young people, have declined globally as well as in the UK (England) in recent years (Cohen et al., 2011; Moliner-Urdiales et al., 2010; Silverman, 2011; Tremblay, Shields, et al., 2010). A study on Canadian children (Tremblay, Shields, et al., 2010) comparing data on 8-10 year olds between 1981 and 2007-2009, and a study on Spanish adolescents (MolinerUrdiales et al., 2010) between 2001/02 and 2006/07, both showed declines in grip strength. Further, a study in England, on 10-year old English children, also found a decline in grip strength over a 10-year secular trend from 1998 to 2008 (Cohen et al., 2011). These results may reflect that activities that produce high physical stresses on the bones to improve bone health and muscle strength are not being incorporated on at least three days per week, as per the vigorous intensity physical activities guidelines for children (Department of Health, 2011). As low muscular strength is significantly associated with key determinants of health (Cheung et al., 2013; Metter et al., 2002; Ortega et al., 2008; Ruiz, Castro-Piñero, et al., 2009; Volaklis et al., 2015), these reported declines are indicative of declines in children's health.

\subsubsection{Body Mass Index}

Reviews of existing field-based tests reported that most physical fitness test batteries for children and young people incorporated body composition, specifically body mass index (BMI), as a component of health-related fitness (Artero, Espaa-Romero, et al., 2011; Castro-Piñero et al., 2010). Body mass index, based on height and weight, is used in many studies that assess paediatric overweight and obesity, as a proxy measure of body size and body fatness, and represents a practical method of assessment in large-scale studies (Artero, Espaa-Romero, et al., 2011; Boddy, Hackett, \& Stratton, 2009, 2010; Castro-Piñero et al., 2010; Olds et al., 2011; Stratton et al., 2007; van Jaarsveld \& Gulliford, 2015). BMI assessment in children is more complex than in adults as a child's BMI changes with age, and growth patterns differ between sexes (Dinsdale, Ridler, \& Ells, 2011). As such, BMI thresholds are often defined using Z-scores which indicate how many units of the standard deviation the BMI is above or below the average BMI for that age group and sex (Dinsdale et al., 2011). Children with high BMI z-score and classified as overweight or obese (Cole, Bellizzi, Flegal, \& Dietz, 2000), are at risk of developing a number of obesity-related conditions (Reilly et al., 2003), including type 2 diabetes (Haines, Wan, Lynn, Barret, \& Shield, 2007), metabolic and cardiovascular complications (Cote, Harris, Panagiotopoulos, Sandor, \& Devlin, 2013; L'Allemand-Jander, 2010) and mental health disorders (Griffiths et 
al., 2010). It is well documented that childhood obesity continues into adolescence and adulthood (Park, Falconer, Viner, \& Kinra, 2012), and having a high BMI in childhood and adolescence may increase the risk of death later in life (Ruiz, Castro-Piñero, et al., 2009). An increased BMI is also associated with lower performance abilities limiting development, which directly affects the capacity of children to acquire skills associated with physical activities (Ceschia et al., 2016). High BMI, and overweight and obesity, ascends from a mismatch between energy intake and energy expenditure, resulting in an accumulation of energy stores, mainly as fat, in the body (Ceschia et al., 2016). Overweight and obesity is associated with a reduction in physical activity levels (Tremblay et al., 2011), and changes in food habits towards high-energy food consumption (Flatt, 2001), as well as, an increase in overall energy intake (Livingstone, 2000). There is compelling evidence that increased physical activity in children is associated with a reduced risk of being overweight or obese (Janz et al., 2009; Moore et al., 2003; Ortega et al., 2011).

Globally the prevalence of overweight and obesity has more than doubled between 1980 and 2014 (World Health Organization, 2018b). However, the prevalence of overweight and obesity varies depending on age, ethnicity, and geographic region. The number of children in Wales classified as overweight and obese represent serious public health problems, with Wales being reported to have the highest rates of childhood obesity in the UK (National Obesity Observatory, 2015). A review presenting a vast amount of data from nine countries (Australia, China, England, France, Netherlands, New Zealand, Sweden, Switzerland and USA) suggested that the rise in the prevalence of overweight and obesity in children aged 2-19 years old has slowed appreciably, or even plateaued, and in some countries slightly decreased (Olds et al., 2011). Specifically, in England, results displayed a plateau in prevalence of overweight or obesity since 2005 (Olds et al., 2011). Further to this, studies in England on 9-11 year old school children (Boddy et al., 2009; Boddy, Hackett, et al., 2010; Stratton et al., 2007), using the SportsLinx project's data archives, have described an initial increase in BMI and prevalence of overweight and obesity from 1998 to 2003/4 (Stratton et al., 2007), then a slowing in the year-on-year increase between 2004 to 2006 (Boddy et al., 2009), and confirmed the levelling off in BMI and prevalence between 2005-2008 (Boddy, Hackett, et al., 2010). In fact, the latest data showed a slight decrease in overweight and obesity prevalence in girls. Regardless of these findings (Boddy et al., 2009; Boddy, Hackett, et al., 2010; Olds et al., 2011; Stratton et al., 2007; van Jaarsveld \& Gulliford, 2015), the presented levels of overweight and obesity are still high. Therefore, continued investment to promote and monitor health- 
related fitness, including body mass index, in children and young people is needed.

Given that investigation into the levels and analysis of trends and inequalities in health-related fitness was warranted, Study 2 aimed to highlight the secular changes in selected health and fitness measures in school children in Wales. In addition, while Study 2 displayed new findings in terms of secular changes in health-related fitness, analyses of inequalities in health-related fitness to predict inequalities in health outcomes were also needed. The aim of Study 3, therefore, was to investigate socioeconomic and sex differences in selected health-related fitness measures in school children in Wales.

\subsubsection{Biological Maturation}

Biological maturation refers to the progression towards the mature (adult) state and varies in timing and tempo (Malina, Bouchard, \& Bar-Or, 2004). It is a process that occurs in all bodily tissues, organs and systems. Significant inter-individual differences exist for the timing, status/level, and tempo of biological maturation (Beunen, Rogol, \& Malina, 2006; Malina et al., 2004; Malina, Rogol, Cumming, Silva, \& Figueiredo, 2015). The timing of biological maturation is defined as the onset of change, or the time when a given maturational event occurs (e.g., growth spurt period, age at menarche); the status/level refers to the magnitude of change or level of maturation at a given chronological age; and tempo denotes the rate of change (Beunen et al., 2006; Malina et al., 2004). These variables are measured and/or observed to provide an indication of a child's progress towards maturity (mature/adult state). Children can be classified as biologically ahead of their chronological age (early-maturing), biologically in line with their chronological age (on-time), or biologically behind their chronological age (late-maturing) (Beunen et al., 2006; Lloyd, Oliver, Faigenbaum, \& Myer, 2014; Malina et al., 2004). This inter-individual variation in biological maturation has an influence on growth, motor performance, and physical fitness (Beunen et al., 2006; Malina et al., 2004), and is an important covariate of the relationships between physical activity, physical fitness, unhealthy/healthy behaviours, body composition, and cardiometabolic risk (Andersen et al., 2006; Katzmarzyk et al., 2012; Malina et al., 2004; Malina \& Kozieł, 2014; Sherar, Cumming, Eisenmann, \& Malina, 2010). Therefore, it is necessary for research studies in children to attempt to control/adjust for biological maturation when feasible.

In children, the status/level and timing of biological maturation are assessed using common methods such as pubertal staging (e.g. Tanner), skeletal age, age at menar- 
che, percentage of adult height, and age at peak height velocity (Beunen et al., 2006; Malina et al., 2004; Malina, Silva, Figueiredo, Carling, \& Beunen, 2012). Due to concerns regarding the invasiveness and the logistical/feasibility challenges of methods (Beunen et al., 2006; Malina et al., 2004), simple non-invasive methods to measure biological maturation have been recommended (Mirwald, Baxter-Jones, Bailey, $\&$ Beunen, 2002). Further, longitudinal data obtained across a period from childhood through to adolescence is required to estimate actual age at peak height velocity. However, this serial longitudinal data is often unavailable. Therefore, the prediction of age at peak height velocity using sex-specific regression equations provides a noninvasive method of assessing biological maturation with the use of one-time anthropometric measures (Mirwald et al., 2002). These equations provide a prediction of years from age at peak height velocity (maturity offset) and are calculated on the known differential timings of growth in height, sitting height and leg length (Mirwald et al., 2002). The maturity offset equations have been widely utilised to define maturity groups and used as a covariate or predictor of physical activity, physical fitness, motor performance, body composition (Andersen et al., 2006; Katzmarzyk et al., 2012; Malina et al., 2004, 2012, 2015; Malina \& Kozieł, 2014; Sherar et al., 2010). Thus, the application of these prediction equations are included wherever possible in the analyses of this thesis.

\subsection{Product/Outcome Measure of Physical Activity - Physical Lit- eracy/ Physical Competence}

The International Physical Literacy Association defines physical literacy (PL) as the motivation, confidence, physical competence, knowledge and understanding to value and take responsibility for engagement in physical activities for life (International Physical Literacy Association, 2016). Such a definition describes the multidimensional and complex nature of PL, highlighting the purported importance of PL as a precursor to physical activity, as well as, being developed through physical activity itself (Edwards et al., 2016). Therefore, given that physical activity has been shown to result in numerous health benefits (Jurbala, 2015), it could be postulated that the promotion of PL is fundamental for physical activity associated health benefits. One of the key elements of PL is physical competence. Much research has looked at physical competence as being the key aspect of physical literacy that is the driving force behind the other aspects of physical literacy and for increasing physical activity participation (Barnett, Lai, et al., 2016; Edwards et al., 2016; Hulteen et al., 2018; Robinson et al., 2015; Stodden et al., 2008; Whitehead, 2010). Therefore, apart from the physical fit- 
ness of children and young people, the development of their motor skills and physical competence should be viewed as an additional and independent physiological factor related to physical activity that requires specific analyses (Guinhouya, 2012).

Physical competence, as a global term relating to the development and performance of human movement, represents an individual's ability to perform skilfully on a wide range of motor tasks, as well as, the movement quality, coordination, and control, underlying a motor outcome (Haywood \& Getchell, 2014; Robinson et al., 2015; Stodden et al., 2008). Physical competence includes not only the acquisition of health- and skill-related components of fitness (e.g. cardiorespiratory fitness, strength, flexibility, agility, balance, coordination, power, reactions and speed), but also movement skills, and plays an important role in the growth and development of children (Barnett, Lai, et al., 2016; Clark \& Metcalfe, 2002; Gallahue et al., 2012; Hulteen et al., 2018; Robinson et al., 2015; Stodden et al., 2008; Whitehead, 2010). In addition, Whitehead (p204; 2010), describes physical competence as "the sufficiency in movement vocabulary, movement capacities and developed movement patterns plus the deployment of these in a range of movement forms." Therefore, even within itself, physical competence is a multidimensional concept. Specifically, movement vocabulary refers to the repertoire of movements that can be performed, and can be expanded through experience and progressive challenge in the deployment of a wide range of movement capacities/skills and movement patterns (Whitehead, 2010). Movement capacities are the integral abilities that make it possible to improve and develop physical competence (Whitehead, 2010). These capacities or skills consist of three interrelated constructs: fundamental or simple movement skills (FMS) (balance, core stability, coordination, speed variation, flexibility, control, proprioception, and power), combined movement (poise, fluency, precision, dexterity, and equilibrium), and complex movement (bilateral coordination, inter-limb coordination, hand-eye coordination, turning, twisting and rhythmic movements, and control of acceleration/deceleration) (Clark \& Metcalfe, 2002; Gallahue et al., 2012; Giblin, Collins, \& Button, 2014; Whitehead, 2010).

The first of these three constructs, fundamental movement skills (FMS), comprise locomotor skills (moving the body in any direction from one point to another), stability skills (balancing the body in one place or while in motion), and object control/manipulative skills (handling or controlling objects with the hand, foot, or an implement) (Gallahue et al., 2012; Giblin et al., 2014; Whitehead, 2010). Although many studies show a weak association between FMS competence and physical activity in young children, this relationship has been shown to strengthen with age (Lubans 
et al., 2010; Robinson et al., 2015; Stodden et al., 2008). Moreover, younger children are more likely to continue to be physically active despite their FMS competence levels, however, older children lacking FMS competence are less likely to demonstrate an active lifestyle (Lloyd, Saunders, Bremer, \& Tremblay, 2014; Lubans et al., 2010; Stodden et al., 2008). Higher levels of FMS competence have also been shown to have a positive relationship with cardiorespiratory fitness (Vlahov, Baghurst, \& Mwavita, 2014), improved academic performance (Jaakkola, Hillman, Kalaja, \& Liukkonen, 2015), and perceived competence and confidence levels (Stodden et al., 2008), whilst having a negative relationship with unhealthy weight status (Rodrigues, Stodden, \& Lopes, 2015). Therefore, many studies indicate that the development and mastery of these foundation skills during childhood, forms a platform for physical competence, and causes a positive trajectory toward physical and psychological health, and sustained physical activity/sporting behaviours, in both the short and long term (Cattuzzo et al., 2016; Lloyd et al., 2014; Lubans et al., 2010; Robinson et al., 2015; Stodden et al., 2008; Van der Fels et al., 2015). The general consensus is that children have the potential to master FMS by 7-8 years of age, with a window of opportunity and FMS developing rapidly between 3 and 8 years of age (Clark \& Metcalfe, 2002; Gallahue et al., 2012). However, development of FMS competence is only the foundation for the acquisition of combined and complex movement competence. While the importance of the acquisition of FMS in relation to an active lifestyle is well-documented, the development of combined and complex movement skills is needed to take part in more advanced physical and sporting activities (Corbin, 2016; Giblin et al., 2014).

The procurement of movement skills and the ability to utilise them to produce movement patterns is essential for the development of physical competence within physical literacy capability (Whitehead, 2010). Movement patterns, described as general (e.g., sending, striking, receiving, running, jumping, rotating), refined (e.g., throwing, dribbling, catching, sprinting, hopping, turning) and specific (i.e. sport-specific movement patterns), are amalgamations of movement that stem from the selection and application of movement skills (Clark \& Metcalfe, 2002; Gallahue et al., 2012; Whitehead, 2010). More refined and specific movement patterns are achieved when FMS, combined and complex movement skills are utilised simultaneously (Clark \& Metcalfe, 2002; Corbin, 2016; Gallahue et al., 2012; Giblin et al., 2014; Whitehead, 2010). There is therefore much need to develop combined and complex movement skills, to take part in more advanced physical activities in a variety of settings (i.e., land, water, air, ice) (Jurbala, 2015; Whitehead, 2010) and movement forms (i.e., adventure, aesthetic, athletic, competitive, fitness and health, interactional/relational) (Whitehead, 
2010), and thus this development is speculated to be imperative to increasing levels of physical competence and physical literacy in maturing children (Clark \& Metcalfe, 2002; Corbin, 2016; Gallahue et al., 2012; Giblin et al., 2014; Tyler et al., 2018; Whitehead, 2010).

Overall, there is a vast array of evidence identifying physical competence as an important component of physical literacy, and, as such, a critical precursor for increasing positive health trajectories, particularly physical activity, across the lifespan (Barnett, Lai, et al., 2016; Edwards et al., 2016; Hulteen et al., 2018; Robinson et al., 2015; Stodden et al., 2008; Whitehead, 2010). Specifically, systematic reviews and longitudinal studies have reported strong evidence for positive associations between physical competence and physical activity levels (Barnett et al., 2009; Holfelder \& Schott, 2014; Lopes et al., 2011; Lubans et al., 2010), health-related fitness (Barnett, van Beurden, et al., 2008; Cattuzzo et al., 2016; Robinson et al., 2015) and perceived competence (Lubans et al., 2010; Robinson et al., 2015), as well as an inverse association with weight status (D'Hondt et al., 2014; Lubans et al., 2010; Robinson et al., 2015), in paediatric populations. Furthermore, studies have shown that enhanced physical competence during childhood tracks across the lifespan by leading to higher levels of physical activity and health-related fitness during adolescence (Barnett et al., 2009; Barnett, van Beurden, et al., 2008; Lopes et al., 2011; Robinson et al., 2015), and by supporting functional independence, general health and quality of life in later life, as well as reducing the risk of all-cause mortality (Hulteen et al., 2018; Paterson \& Warburton, 2010; Sigmundsson et al., 2016). Thus, enhanced physical competence in children and young people is foundational for physical activity promotion and associated health benefits, with transferable value throughout the life course.

As physical literacy has been highlighted as an important precursor of physical activity, with physical competence being a critical factor in enhancing physical literacy, continued investment into developing physical competence in children is required. As such, Study 5 aimed to quantify the relationships between socio-ecological factors and physical competence to inform future intervention strategies that aim to promote physical competence in children and young people.

\subsubsection{Measurement of Physical Competence}

The distinct discipline of motor development (physical competence) gained widespread attention in the 1970s, with an emphasis being placed on the development of various assessments in the decades to follow (Cattuzzo et al., 2016). Nonetheless, developing 
an accurate assessment is still one of the primary themes related to physical competence found in the literature, evidencing the complexity of this task and showing that significant gaps exist in established assessments (Lundvall, 2015). There is a need to develop valid tools that effectively and efficiently assess physical competence in order to evaluate whether programmes are successful (Corbin, 2016). Physical competence can be evaluated by process- or product-based assessments (Bruininks \& Bruininks, 2005; Cairney et al., 2018; Henderson et al., 2007; Physical and Health Education Canada (PHE Canada), 2014; Ulrich, 2000). Primarily process-based assessments (e.g., the Test of Gross Motor Development (TGMD-2), CS4L: Physical Literacy Assessment for Youth Fun (PLAYfun), Passport for Life: Movement Skills Assessment) measure how children move and provide qualitative information on the technique of the movement patterns (Hardy, King, Farrell, Macniven, \& Howlett, 2010). This type of assessment can be sensitive to assessor experience and subjectivity (Schoemaker, Niemeijer, Flapper, \& Smits-Engelsman, 2012). On the other hand, assessments that are primarily product-based (e.g., the Movement Assessment Battery for Children-2 (MABC-2), the Bruininks-Oseretsky Test of Motor Proficiency, Second Edition (BOT-2)) are usually quantitative and focus on the outcome of the movement (Logan, Barnett, Goodway, \& Stodden, 2017), but potentially lack the sensitivity needed to identify individual differences in movement abilities (Giblin et al., 2014). The equivocal relationship between process- and product-based assessments of physical competence has resulted in the use of combined assessments for measuring physical competence (Logan et al., 2017; Robinson et al., 2015; Rudd et al., 2016). The assessment of physical competence can be formative or summative. Specifically, formative assessments measure current levels of performance to identify a baseline and the individual needs of children, enabling the development of an educational programme catered to those children, whereas summative assessments are used to measure progress of a child at the end of a period of education (Gallahue $\&$ Donnelly, 2007).

Most existing land-based movement skill assessments involve the performance of discrete skills in isolation (e.g., TGMD-2/3 (Ulrich, 2000), BOT-2 (Bruininks \& Bruininks, 2005), MABC-2 (Henderson et al., 2007), CS4L: PLAYfun (Cairney et al., 2018), Passport for Life: Movement Skills Assessment (Physical and Health Education Canada (PHE Canada), 2014)). Balancing, running, hopping, jumping, throwing, dribbling, catching and sprinting are common skills that are assessed within these existing movement skill assessment tools (Bruininks \& Bruininks, 2005; Gallahue et al., 2012; Henderson et al., 2007; NSW Department of Education and Training, 2000; 
Ulrich, 2000). This static testing environment limits transferability and applicability to multi-skill and sport environments and does not assess combined and complex movement skills (Giblin et al., 2014). It has been suggested that considering skills in isolation ignores a constraints-based approach (Newell, 1986), in which environmental constraints are taken into account, and by doing so this approach is not 'authentic'. An authentic environment is one that is developmentally-appropriate and considers the interaction of the individual and the environment, as well as the specified movement skill (Barnett, Stodden, et al., 2016; Newell, 1986). Performance of movement skills in isolation does not incorporate the measurement of an individuals' ability to alter and combine movement skills according to the task at hand and the environment, both of which are important traits to advance physical competence and progress one's physical literacy (Whitehead, 2010). Finally, assessments that measure skills in isolation have also been criticised for being time- and resource-intense (Giblin et al., 2014; Wiart \& Darrah, 2001).

Recently, the Canadian Agility Movement Skill Assessment (CAMSA) was developed and validated to assess physical competence in 8-12-year-old children (Longmuir et al., 2017). This assessment requires a series of seven movement tasks (twofooted jump, side slide, catch, throw, skip, hop, and kick) to be completed in a continuous dynamic obstacle course to create a more authentic environment and to assess combined and complex movement skills. Performances are assessed using the time taken to complete an obstacle course consisting of 14 process/technique- and product/outcome-based criteria (Longmuir et al., 2017). This assessment has shaped the way towards assessing movement skills in a dynamic fashion. However, there are noteworthy design limitations of the CAMSA, and as such, an assessment targeting older aged children and adolescents (10-14 years), with a more balanced design, is warranted. Therefore, Study 4 aimed to develop and assess the validity and reliability of a more balanced dynamic assessment tool to measure physical competence in children and young people. 


\section{General methodology}

The methods described in this chapter are the field-based methods of data collection common to the studies within this thesis. Detailed methods, sample sizes and statistical analyses specific to each study are presented in the relevant chapters and appendices.

\subsection{Ethical Approval}

Ethical approval for Studies 2-5 was granted with the guidelines and policies of the Swansea University College of Engineering Research Ethics and Governance Committee (REC numbers: PG14/2014/007; PG/2014/020; PG/2014/37; PG/2014/39). Information sheets were developed for children, parents/ guardians and school staff/ head teachers, and prior to study involvement, written informed parent/ guardian and school/ head teacher consent and child written assent was obtained (see Appendix XIII).

\subsection{Instruments and Procedures}

\subsubsection{Swan-Linx Fitness Fun Day: Field-Based Fitness Measures}

Fitness tests were taken from the EUROFIT fitness test battery (Council of Europe, 1993), and completed at a Fitness Fun Day using standard techniques (Taylor, Hackett, Stratton, \& Lamb, 2004). The fitness tests reported in this chapter are those used in the thesis, but do not cover the full Fitness Fun Day measures. Further information on the full Fitness Fun Day measures, and the Standard Operating Procedures for administering the fitness measures are provided in Appendix II.

Appropriately trained and experienced Swansea City Council Active Young People Officers and Sports Science postgraduates led the Fitness Fun Day testing, with the help of undergraduate Sport and Exercise Science students and Sport Wales Young Ambassadors. Guides on each of the testing procedures were positioned at each testing station for reference. Prior to completing the tests, participants completed a warm up of 8-10 minutes and then assigned into groups. Groups then rotated around the testing stations. Due to the size of the testing facilities and variations in the number of children from each school (20-100 children), it was not possible to have all children complete the fitness tests in the same order. 
Body Mass: Each participant's body mass was measured to the nearest $0.1 \mathrm{~kg}$ using portable weighing scales [Seca 876, Hamburg, Germany]. Participants wore light, athletic clothing and removed their shoes, pull-overs (if wearing one) and anything in their pockets.

Stature: Participants removed footwear, looked straight ahead and kept their head level, while standing upright against a stadiometer [Seca 213 portable stadiometer, Hamburg, Germany]. The vertical distance between the floor and top of the skull was measured to the nearest $0.1 \mathrm{~cm}$.

Sitting Stature: Participants removed their footwear and sat on a sitting stature stadiometer [Harpenden Sitting Height Table, Holtain Ltd, Pembrokeshire, UK]. Participants' feet were positioned on a rung that supported their legs and allowed a right angle at the knee joint. Participants placed their hands on their legs, kept their head level and sat as straight and tall as possible. The vertical distance between the top of the skull and the base of the sitting surface was measured while the participants took a deep breath in.

Body Mass Index and Classification of Weight Status: Body Mass Index (BMI) was calculated $\left(\mathrm{BMI}=\right.$ body mass $(\mathrm{kg}) /$ stature $\left.^{2}\left(\mathrm{~m}^{2}\right)\right)$ and BMI z-scores were derived using the UK 1990 growth reference curves (Cole, Freeman, \& Preece, 1995). Classification and prevalence of participants' being underweight, healthy weight, overweight or obese, were calculated using age and sex specific BMI cut-off points (Cole et al., 2000). Sex-specific maturation offset regression equations using interactions between body mass, stature, sitting stature, lower limb length and decimal age, were used to predict participants' age from peak height velocity (Mirwald et al., 2002). This calculation was used as a proxy measure of biological maturation.

$20 m$ Multistage Shuttle Run Test: The 20m Multistage Shuttle Run Test (20m MSRT) involved continuous running between two lines $20 \mathrm{~m}$ apart, in time to recorded beeps from a CD. In line with a standardised lap scoring protocol of the 20m MSRT (Riddoch, 1990), the test score achieved by the participant was the number of shuttles completed before the subject either withdrew voluntarily from the test, or failed to reach the end line on two consecutive beeps. To ensure a consistent pace and to encourage participants to run to exhaustion, a researcher completed the test with the participants. 
Handgrip Strength: Using hand dynamometers [Takei Corp Ltd., Tokyo, Japan], participants' handgrip strength was measured in kilograms. Participants held the dynamometer with their arm fully extended above their head and squeezed the dynamometer with maximum isometric effort for $5 \mathrm{~s}$, whilst bringing it down slowly towards their side.

\subsubsection{Child Health and Activity Tool: Online Questionnaire}

The Child Health and Activity Tool (CHAT) questionnaire, akin to the SportsLinx Lifestyle Survey (Taylor et al., 2004), was developed at Swansea University. The CHAT is an interactive, online questionnaire and is available in English and Welsh. The first section of the CHAT consists of questions that require children to recall what they did the previous day. The second section of the CHAT consists of asking how many times a week a child engaged in certain behaviours (e.g., how many times a week they were sedentary for $>2$ hours). The third section includes general lifestyle questions. Participants completed the CHAT in a classroom or computer suite at school, supervised by teachers and 3-4 postgraduates or Active Young People Officers. A full paper copy of the questionnaire is shown in Appendix III and the Standard Operating Procedure for administering it is shown in Appendix IV.

\subsubsection{The Dragon Challenge: Physical Competence Measure}

The Dragon Challenge (DC) consists of nine tasks (Balance Bench, Core Agility, Wobble Spot, Overarm Throw, Basketball Dribble, Catch, Jumping Patterns, T-Agility, and Sprint), which require the application of a different combination of fundamental, combined and complex movement skills, to form refined and specific movement patterns. The order of the tasks is standardised, but the challenge is performed under the illusion that the order is random, except for the final task, which is always the sprint.

Participants observed a demonstration of each DC task and then the full DC. Participants were given two practice attempts at each challenge task, but they did not practise the challenge in full. One trained administrator then led the participants in the DC, while at least one trained assessor scored the participants in accordance with the instructions specified within the DC manual (see Appendix V). In particular, one point was given for each of the technical/process criterion and two points for each outcome/product criterion successfully demonstrated by participants. Time taken to complete the DC was also converted to a score (higher scores for faster time). Each of these constructs (technique, outcome, and time) was scored out of 18 in order to be equally weighted, and then summed to give a total score. For further information 
on the DC assessment including equipment list, layout schematic, descriptions of the assessment and video resources, see Appendices V and VI.

\subsection{Conclusion}

This general methodology has outlined the methods shared by many of the studies in this thesis. Specific methods for each individual study will be discussed within the relevant chapter. 


\section{Thesis Map}

Study

Outcomes

\begin{tabular}{|c|c|c|c|}
\hline 1 & $\begin{array}{l}\text { Results from the } \\
\text { Active Healthy Kids } \\
\text { Wales } 2016 \text { Report } \\
\text { Card on Physical } \\
\text { Activity for Children } \\
\text { and Youth }\end{array}$ & Aim & $\begin{array}{l}\text { To consolidate and translate } \\
\text { research related to physical } \\
\text { activity among children and } \\
\text { youth in Wales and to } \\
\text { synthesise national } \\
\text { surveillance data from } \\
\text { several surveys in order to } \\
\text { assign grades to physical } \\
\text { activity/inactivity behaviours } \\
\text { and influences indicators, as } \\
\text { well as to examine trends or } \\
\text { inequalities in the data. }\end{array}$ \\
\hline & & Key Findings & \\
\hline 2 & $\begin{array}{l}\text { Ten-year Secular Changes in } \\
\text { Selected Health and Fitness } \\
\text { Parameters of 10-11 Years Old } \\
\text { Swansea School Children - 2003- } \\
2013\end{array}$ & Key Findings & \\
\hline 3 & $\begin{array}{l}\text { A Cross-Sectional Study on the } \\
\text { Socio-economic and Sex } \\
\text { Differences in Health-Related } \\
\text { Fitness Measures in School } \\
\text { Children }\end{array}$ & Key Findings & \\
\hline 4 & $\begin{array}{l}\text { A Dynamic Assessment of } \\
\text { Children's Physical Competence: } \\
\text { The Dragon Challenge }\end{array}$ & $\begin{array}{c}\text { Aim } \\
\text { Key Findings }\end{array}$ & \\
\hline 5 & $\begin{array}{l}\text { Youth Physical Competence } \\
\text { Promotion Model: A Quantitative } \\
\text { Investigation into Modifiable } \\
\text { Factors }\end{array}$ & Key Findings & \\
\hline
\end{tabular}




\section{Study 1}

\subsection{Results from the Active Healthy Kids Wales 2016 Report Card on Physical Activity for Children and Youth: "Is Wales Turn- ing the Tide on Children's Inactivity?"}

* This chapter is a published manuscript:

Tyler, R., Mannello, M., Mattingley, R., Roberts, C., Sage, R., Taylor, S. R., .. . Stratton, G. (2016). Results From Wales' 2016 Report Card on Physical Activity for Children and Youth: Is Wales Turning the Tide on Children's Inactivity? Journal of Physical Activity and Health, 13(11 Suppl 2), S330-S336. https://doi.org/10.1123/ jpah.2016-0309

\subsection{Background}

Wales has a population of approximately 3 million people of which $\sim 702,600$ are children and young people (0-19 years of age) (Statistics Wales, n.d.). The majority of children in Wales are insufficiently active leaving Wales in the bottom quartile globally for physical activity levels in school-aged children (Inchley et al., 2016). Inequalities in physical activity also exist: twice as many boys are sufficiently active as girls, physical activity levels decline with age, differences exist between ethnic groups and there is a social gradient where activity follows socio-economic status (Inchley et al., 2016). In addition, cardiorespiratory fitness levels of children in Wales are amongst the lowest in Europe (Tomkinson \& Olds, 2007). Further, recent data found that less than half of 3000 9-11 year old children assessed achieved a healthy level of overall health-related fitness (Tyler, Mackintosh, Brophy, et al., 2015). In addition, obesity levels of children in Wales are the highest in the UK (Bailey, 2015) and amongst the highest globally (Inchley et al., 2016).

Conversely, Wales has a good record of supporting physical activity through policies and strategies. For example, Sport Wales have a mission to get "every child hooked on sport for life" (Sport Wales, n.d.-d), and support this with an emphasis on physical literacy through physical education and sport programming (Sport Wales, n.d.-a). In 2001 Wales was the first country to make statute article 31 of the United Nations Convention on the Rights of the Child (UNCRC) recognising the rights of children to play ("United Nations Convention on the Rights of Children - Rights - Children in Wales," n.d.), and also the first country to have an Active Travel Act ("Active Travel (Wales) Act 2013,” 2013). 
This is the second Active Healthy Kids (AHK) Wales Report Card following the inaugural 2014 version http://www.swansea.ac.uk/activehealthykidswales/. Swansea University coordinated the work, supported by a national network of academics and professionals from sport, play, transport, public health, sport and exercise science, and education sectors. These constituents formed the research working group (RWG).

The purpose of this manuscript is to summarise the findings of the AHK Wales 2016 Report Card. Data from a number of surveys collected from 2013 to 2015 were synthesised to assign grades to each quality indicator. Where available, data as far back as 2002 were used for the analysis of trends.

\subsection{Methods}

The RWG consisted of 11 members. The lead academic directed the development of the Report Card contents and RWG membership. The RWG comprised of the lead academic (Gareth Stratton), post-graduate researchers, academics, and professionals with expertise in physical activity and access to national data sources. The academic lead secured funding and outlined the programme of work. The lead post-graduate researcher (Richard Tyler) was responsible for data sourcing, synthesis and subsequent presentation to the RWG for interpretation, discussion and grading. The lead researcher was also responsible with the academic lead to produce the Report Card and web site materials. All members of the RWG collectively allocated grades to indicators. The methodology used was in accordance with the AHK Global Matrix (Tremblay et al., 2014) and was used in RWG meetings to evaluate the synthesised summary statistics, make informed decisions and assign grades.

Ten indicators were assessed, an additional two (school and physical literacy) to the eight included in the AHK Wales 2014 Report Card. School was added to be consistent with other countries and physical literacy, 'the motivation, confidence, physical competence, knowledge and understanding to value and take responsibility for engagement in physical activities for life' (Weinberg, Talbot, \& Whitehead, 2013), because of the emphasis placed on it by Sport Wales and the Education department of Welsh Government. The indicators assessed were: 1. overall physical activity levels; 2. organised sport participation; 3 . active and outdoor play; 4. active transportation; 5. sedentary behaviours; 6 . physical literacy; 7. family and peer influence; 8. school; 9. community and the built environment; 10 . national government policy, strategies and investments. A number of surveys were selected for sources of data in the current 
Report Card; these are presented in Table 1. The data sources used were the most recent (2013-2015) at the time of the grading process. In the first instance, surveys that were representative of children in Wales were selected. Where nationally representative data were not available the best available data were used. For the national government policy, strategy and investment indicator, a number of policy documents, strategy documents and other guidance that were publicly available were considered to inform the RWG during the grading process ("Physical Activity and Nutrition Network - Fitness and Health Info Resources," n.d.). 
Table 1. Data sources used to inform each grade allocated to the 10 indicators of the Active Healthy Kids Wales Report Card

\begin{tabular}{llll}
\hline Data Source & Sample Size & Age Range & Indicator* \\
\hline $\begin{array}{l}\text { Sport Wales: SSS (2015) } \\
\text { (Sport Wales, 2015c) }\end{array}$ & 115,398 children & $7-16$ & $2,3,4,7,8$
\end{tabular}

Sport Wales: FESS (2015)

(Sport Wales, 2015b)

4,568 young people

$16-20+\quad 2$

HBSC Wales (2013/14)

(Welsh Government, 2015a)

9,055 children

$11-16$

$1,4,5$

WHS (2014)

(Welsh Government, 2015c)

2,800 children; 14,170 adults $\quad 4-15 ; 16+\quad 1,7$

NSW (2014/15)

(Welsh Government, 2015b)

14,285 parents of children $\quad 1-15 \quad 4,9$

Sport Wales: AAS (2014)

(Sport Wales, 2015a)

13,145 adults

$15+$

7

Sustrans Wales: HUS (2014/15)

("Sustrans Wales, Sustrans Cymru," n.d.)

4356 children $\quad 7-16 \quad 4$

\section{LVSO (2015)}

("Little Voices Shouting Out

Report," 2015)

724 children

$7-11$

3

Beth Nesa? | What Next? (2015)

(Beth Nesa? What Next? 2015, 2015)

5961 children \& young people $3-18+\quad 9$

Sport Wales: DC Report (2015)

Unpublished

1600 children

$11-12$

6

Note. The grade for indicator 10 was allocated using documents and guidance not in this table. Abbreviations: SSS, School Sport Survey; FESS, Further Education Sport Survey; HBSC, Health Behaviour in School-aged Children; WHS, Welsh Healthy Survey; NSW, National Survey for Wales; AAS, Active Adult Survey; HUS, Hands Up Survey; LVSO, Little Voices Shouting Out; DC, Dragon Challenge.

* Number relates with the order in which the indicators were listed in the Methods. 
The RWG met once a month to discuss, evaluate and critique data for each of the 10 indicators. During the meetings, data were reviewed in terms of; representativeness of the survey, methodology of data collection, age range of participants, sample size, and the reporting of subgroups such as sex, age, disability and socio-economic status. Commensurate with the AHK Global Matrix (Tremblay et al., 2014), specific benchmarks for each indicator and the grading framework of: A, we are succeeding with a large majority of children and youth (81-100\%); B, we are succeeding with well over half of children and youth $(61-80 \%)$; $\mathrm{C}$, we are succeeding with about half of children and youth (41-60\%); D, we are succeeding with less than half, but some, children and youth $(21-40 \%)$; F, we are succeeding with very few children and youth $(0-20 \%)$, were used in assigning grades to each indicator. Grades were assigned a ' + ' or '-' to indicate a trend or inequality in the data. A consensus approach was used to reach a decision and assign a grade to each indicator.

\subsection{Results}

Grades assigned to each indicator by the RWG are presented in Table 2, and the front cover is illustrated in Figure 2. Results are discussed herein on an indicator basis.

Table 2. Grades according to physical activity indicator in the Active Healthy Kids Wales 2016 Report Card on physical activity for children and youth

\begin{tabular}{lc}
\hline Indicator & Grades \\
\hline Overall Physical Activity Levels & D- \\
Organised Sport Participation & $\mathrm{C}$ \\
Active and Outdoor Play & $\mathrm{C}$ \\
Active Transportation & $\mathrm{C}$ \\
Sedentary Behaviours & D- \\
Physical Literacy (Physical Competence) & $\mathrm{INC}$ \\
Family and Peer Influence & D+ \\
School & B \\
Community and the Built Environment & $\mathrm{C}$ \\
National Government Policy, Strategies and Investments & B- \\
\hline
\end{tabular}

Note. The grade for each indicator is based on the percentage of children and youth meeting a defined benchmark: $A$ is $81 \%$ to $100 \%$; $B$ is $61 \%$ to $80 \%$; $C$ is $41 \%$ to $60 \%, D$ is $21 \%$ to $40 \%$; $F$ is $0 \%$ to $20 \%$; INC is Inconclusive data. 


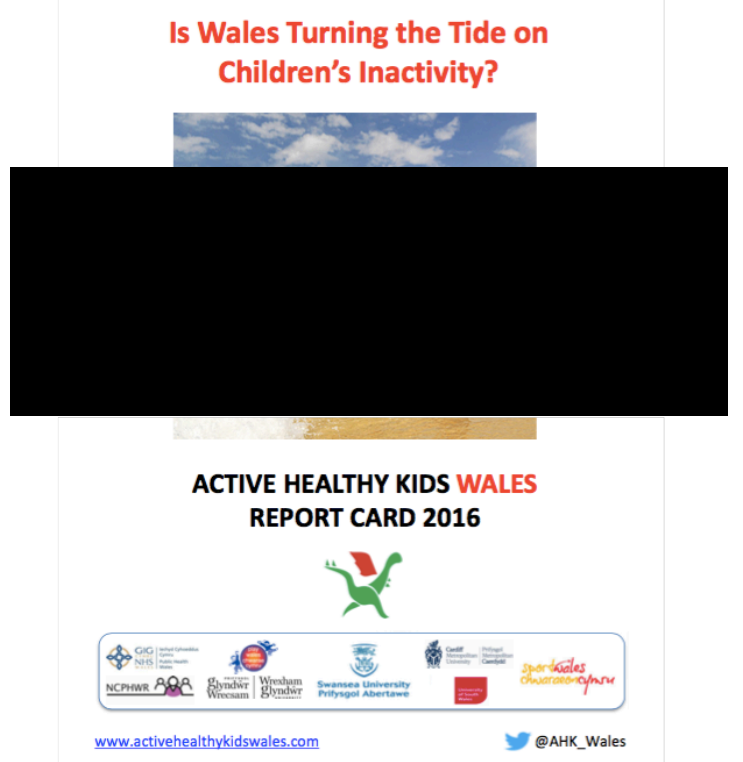

Figure 2. Front cover of the Active Healthy Kids Wales 2016 Physical Activity Report Card

The cover story for the report card is: "Is Wales Turning the Tide on Children's Inactivity?" With over 100 beaches and over 750 miles of coastline in Wales, only $42 \%$ of children used the beach or sea at least once in the past year for exercise (Sport Wales, 2015c). Furthermore 90\% of the population live within 30 minutes of the coast. Subsequently "turning the tide" captures meaning in the Welsh context and the aim of this Report Card is to "turn the tide on children's inactivity" and advocate national efforts to increase children's physical activity and reduce their sedentary time. Turning the tide on children's inactivity in Wales should be used to influence and inform policies and strategies, support structures, investments and opportunities that help promote sound health, growth and development in the nation's children and young people.

\subsubsection{Overall Physical Activity Levels}

Grade: D-. Two nationally representative data sources were used to assign an overall physical activity grade. Self-report data showed that $15 \%$ of children and young people aged 11-15 years old met the recommendation of at least 60 minutes of moderate to vigorous intensity physical activity (MVPA) every day of the week (Welsh Government, 2015a). Further analysis revealed that boys (20\%) were more active than girls $(11 \%)$ across all age groups and socio-economic status. Overall, there was little difference in MVPA by socio-economic status (15\% low, 13\% medium, 17\% high) or geographic regions (17\% North, 16\% West, 17\% East, 15\% Southeast, $15 \%$ Central south). In terms of changes over time there was a small downward trend in 
MVPA between 2006 and 2014 (21\% in 2006, 19\% in 2010, 15\% in 2014). Proxy report (parent) data further showed that $35 \%$ of children aged $4-15$ years participated in MVPA for at least 1 hour every day and, although sex differences persisted (40\% of boys, 29\% of girls), there was no significant change between 2007 and 2014 (Welsh Government, 2015c). D- was assigned to physical activity as the proportion of children sufficiently active was between 20 and $39 \%$ and inequalities existed between sub groups. There was no change in grade from the AHK Wales 2014 Report Card.

\subsubsection{Organised Sport Participation}

Grade: C. In line with Sport Wales' Vision, the proportion of children 'hooked on sport' (children who take part in sport on three or more occasions a week in an extracurricular (school-based) or a community club setting) captured in the School Sport Survey (Sport Wales, 2015c) was used as the benchmark for grading. Of children aged 7-16 years old, $48 \%$ took part in sport on three or more occasions a week (Sport Wales, 2015c). These data showed an upward trend from 27, 40, and 48 percent in 2011, 2013 and 2015, respectively. Moreover, similar proportions of primary (49\%) and secondary (48\%) age school children took part in sport on three or more occasions a week although more boys (52\%) met the criteria than girls (44\%). Even though there were differences by age (52\% of 10-12 year olds, $45 \%$ 14-16 year olds), ethnicity (White 49\%, Mixed Race 52\%, Black 52\%, Asian 36\%, Other 39\%), disability (40\% with a disability, $49 \%$ without) and socio-economic status (43\% low, $54 \%$ high), there was an overall increase in the proportion of children who took part in sport on three or more occasions a week between 2013 and 2015 for boys and girls (Sport Wales, 2015c). The Further Education Sport Survey of students aged 16 and over also showed that $49 \%$ took part in sport three or more times per week, in any setting (Sport Wales, 2015b). Of these further education students 52\% of $16-19$ and $37 \%$ of 20+ year olds met the criteria for taking part in sport. The RWG concluded that the increase in the proportion of children who took part in sport three or more times per week, and the finding that at least $40 \%$ of children in the vast majority of subgroups (sex, ethnicity, socio-economic status and disability) met this criterion, was sufficient to warrant an increase in the grade from a C- in 2014 to a C in the 2016 Report Card.

\subsubsection{Outdoor and Active play}

Grade: C. The grade for this indicator has not changed since 2014 although more data were available to support the 2016 grade. In the previous Report Card, 'use of the park' was used solely to establish the grade for this indicator. In the 2016 Report Card, the RWG agreed that the proportion of children using a variety of places 
for play should be considered. Data on the proportion of children and youth using 'streets near home or school' (43\%), 'park' (61\%), 'playground not at school' (38\%), 'playing fields at school' (48\%), 'playing fields not at school' (50\%) and 'beach/sea' (42\%) for places of physical activity/play in the previous year were synthesised to give an overall proportion of $47 \%$ of children and youth using at least one of these places (Sport Wales, 2015c). The Little Voices Shouting Out survey of children aged 7-11 years from across Wales reported that 18\% spent 3 hours, 15\% 4 hours and $27 \%$ more than 4 hours playing outside on a normal day ("Little Voices Shouting Out Report," 2015). The RWG concluded that $60 \%$ of children reporting spending $\geq 3$ hours outside playing was commensurate with a $\mathrm{C}$ grade.

\subsubsection{Active Transportation}

Grade: C. The largest available survey of active transportation in 7-16 year old children showed that $40 \%$ reported they either walked, cycled, scooted or skated to school (Sport Wales, 2015c). In a survey of parents of primary and secondary school children (Welsh Government, 2015b), 49\% of primary school children and 35\% of secondary school pupils walked to school regardless of distance. Data on children and young people aged 11-16 years showed that $32 \%$ (34\% boys, $31 \%$ girls) completed the main part of their journey to school by walking or cycling (Welsh Government, 2015a). This data indicates that little has changed in the proportion of children and young people who actively travelled to school between the years 2006 to 2014 (Welsh Government, 2015a). Data provided by Sustrans Wales ("Sustrans Wales, Sustrans Cymru," n.d.), showed that 53.7\% of children aged 7-16 years travel to school either by walking, cycling, or scooting/skating, and levels of children travelling to school by walking, cycling, or scooting/skating were consistent for the last 4 years of data collection. There was reassuring consistency across data sources for active transportation and this indicator was assigned a $\mathrm{C}$ grade.

\subsubsection{Sedentary Behaviours}

Grade: D-. On a weekday, 53\% of children and young people spent $2+$ hours a day playing games on an electronic device, $64 \%$ spent $2+$ hours a day using an electronic device for purposes other than gaming, and $68 \%$ spent $2+$ hours a day watching entertainment on a screen (Welsh Government, 2015a). On the weekend, the proportion of children and young people who spent $2+$ hours a day playing games on an electronic device, using an electronic device for purposes other than gaming, and watching entertainment on a screen increased to $65 \%, 71 \%$, and $80 \%$, respectively (Welsh Government, 2015a). During weekdays, a higher proportion of boys spent 2 or more hours 
a day playing games on an electronic device and watching entertainment on a screen compared to girls. On the other hand, a higher proportion of girls spent more than 2 hours a day using an electronic device for purposes other than gaming. During the weekend, the sex differences in the proportion playing games on an electronic device and using an electronic device for purposes other than gaming remained the same as on weekdays, but there were similar proportions of both boys and girls spending more than 2 hours a day watching entertainment on a screen. Findings for both weekdays and weekends were consistent across all socio-economic status, and findings for playing games on an electronic device were similar across age groups. Use of electronic devices for purposes other than gaming and watching entertainment increased with age for boys and girls. The RWG concluded that the increase in sedentary behaviour on the weekend compared to weekdays and differences according to sex and age, justified the decrease from a grade D in 2014 to a D-.

\subsubsection{Physical Literacy}

Grade: Inconclusive. Wales has recently designed a national measure, the Dragon Challenge, for the physical competency domain of physical literacy for children aged 8-14 years. The Dragon Challenge has produced pilot data on children across Wales and initial results suggest that continued investment in developing skilled movements in children is required ("Dragon Challenge Circuit Video," 2015). The RWG decided to grade physical literacy as inconclusive based on the very recent development of the measurement tool for physical competency and the lack of available data on the other domains of physical literacy.

\subsubsection{Family and Peer Influence}

Grade: D+. Results from the Sport Wales Active Adult Survey (2014) found that $41 \%$ $(46.3 \% \mathrm{M}, 35.1 \% \mathrm{~F})$ of the adult population took part in sport on 3 or more occasions per week, with the equivalent figure being 39\% in 2012 (Sport Wales, 2015a). Findings from the Welsh Health Survey (2014), revealed that 3 out of 10 adults (38\% M, $23 \% \mathrm{~F}$ ) reported being physically active for $>30$ minutes on 5 or more days a week and there has been no increase in physical activity levels in adults since 2003 (Welsh Government, 2015c). Analysis of the School Sport Survey data (Sport Wales, 2015c) found there is an increased probability of children taking part in sport on three or more occasions a week if their parents or friends are also involved in sport, with a $32 \%$ increased chance if their dad, $27 \%$ increased chance if their mum and $23 \%$ increased chance if their friends were also involved in sport (Sport Wales, n.d.-c). The same survey found that $27 \%$ of mothers, $36 \%$ of fathers, $39 \%$ of brothers, $31 \%$ of sisters, 
$35 \%$ of other family members and $64 \%$ of children's friends (peers) took part in sport (Sport Wales, 2015c). Further, 18\% and 26\% of these children took part in sport with their mother and father, respectively. In addition, $25 \%$ of children took part with their brother, $19 \%$ with their sister, $20 \%$ with other family members, and $68 \%$ with their peers (Sport Wales, 2015c). Although there was little change in the participation in sport and physical activity levels of adults, the additional evidence demonstrating a parental/peer influence on children's participation in sport, which was absent in the 2014 Report Card, prompted the RWG to assign a grade of D+ compared to D in the 2014 Report Card.

\subsubsection{School}

Grade: B. To grade this indicator, school Physical Education Coordinators and children's responses in the School Sport Survey (2015) were synthesised (Sport Wales, 2015c). The survey provided data on: percentage of schools that offer physical activity opportunities (excluding PE) to the majority of their pupils, percentage of schools with sufficient facilities/equipment to support pupils' physical activity and sport, percentage of schools where teachers were confident in delivering quality PE lessons, and percentage of children that believe PE lessons and school sport help them to have a healthy lifestyle (Sport Wales, 2015c). In total, 60\% of primary schools and $80 \%$ of secondary schools provide a wide variety of extracurricular sport and dance opportunities to all pupils. This provision includes health, fitness and wellbeing activities, competitive activities, creative activities, and adventure activities. On average, primary schools provide 11 different extracurricular activities for pupils and secondary schools provide 16 . Physical education staff reported that $70 \%$ of primary and $62 \%$ of secondary schools agreed that the school had sufficient access to facilities to provide sport, and that $54 \%$ of primary and $50 \%$ of secondary schools owned sports facilities that were of high quality. Further, $73 \%$ of primary and $71 \%$ of secondary schools agreed that they had access to sufficient equipment to provide quality physical education and sport, and $77 \%$ of primary and $85 \%$ of secondary schools reported teachers were confident in delivering quality physical education lessons. From the children's perspective, $62 \%$ of children reported that physical education lessons and school sport had really helped them to have a healthy lifestyle. In summary, schools were well resourced and the RWG assigned B to this indicator.

\subsubsection{Community and the Built Environment}

Grade: C. Data from the National Survey for Wales found 54\% and 38\% of parents with a child aged 1-10 and 11-15 years, respectively, were satisfied with the play 
facilities in their local area (Welsh Government, 2015b). Of parents with a child aged 1-10 years who were dissatisfied, $83 \%$ reported a lack of suitable outdoor public places for their child to play, whereas $64 \%$ reported a lack of suitable indoor spaces. Additionally, of the parents with a child aged 11-15 years who were dissatisfied, $77 \%$ cited a lack of suitable outdoor public places, $79 \%$ believed there was a lack of indoor public places, and $64 \%$ believed there were too few clubs or organised activities. Consultations with children, young people (aged 3-18+ years) and their parents showed that 'more places to play' was the highest priority (49\%) for children aged 3-7 (Beth Nesa? What Next? 2015, 2015). Other common priorities for this age group were tackling poverty (29\%), improving child safety (28\%) and tackling bullying (28\%). For children aged 7-11 years, stopping bullying was the highest priority (55\%) (Beth Nesa? What Next? 2015, 2015). The next two highest priorities for this age group were helping children and families in poverty (47\%) and making local areas safer for children $(41 \%)$. The most common priority (53\%) for young people aged 11-18 was tackling bullying (Beth Nesa? What Next? 2015, 2015). More than $40 \%$ of young people reported protecting children from violence and abuse at home, more facilities in the local area, and help for children and families in poverty as their highest priorities. Across all data reported, priorities did not vary substantially by age, sex or region for all age groups. The grade for community and the built environment has decreased from a B in the 2014 Report Card, to a C in 2016. This is due to the proportion of parents being dissatisfied with the play facilities in their local area.

\subsubsection{National Government Policy, Strategies and Investments}

Grade: B-. After extensive discussions, the RWG concluded that the grade would remain the same as in 2014. The grade was based on the findings that: a) at Governmental and quasi non-governmental levels there is still evidence of leadership and commitment to provide physical activity opportunities for all children and youth; $b$ ) there are allocated funds and resources for the implementation of strategies to promote physical activity for all children and youth; c) the Government has demonstrated progress through the key stages of public policy making; d) Wales still has a number of key national and organisational policies that include physical activity ("Physical Activity and Nutrition Network - Fitness and Health Info Resources," n.d.) and are making cross-government efforts to integrate them. With the exception of the proportion of children and young people who took part in sport on three or more occasions a week, the RWG also recognised that policies and strategies had not yet increased physical activity and that inequalities in physical activity remain. 


\subsection{Discussion}

The results of the AHK Wales 2016 Report Card demonstrate that significant efforts need to be made to change children's inactive lifestyles and related choices. Further, using recommendations from the National Institute for Health and Care Excellence ("NICE - The National Institute for Health and Care Excellence," n.d.), the best available evidence was synthesised and showed that the majority of children in Wales were insufficiently active (did not meet recommendation of at least 60 minutes of MVPA every day of the week), and spent excess time ( $>2$ hours) in sedentary behaviour (Sport Wales, 2015c; Tyler, Mackintosh, Brophy, et al., 2015; Welsh Government, 2015a, 2015c). The most recent HBSC international report (2016) included summary statistics on physical activity and TV watching from 42 countries worldwide (Inchley et al., 2016). These statistics demonstrate that Wales has one of the largest differences in number of boys who achieved 60 minutes of MVPA between high and low affluence groups. The differences for girls were not as marked and were more representative of other countries. A similar picture emerged for TV watching but there were negligible sex differences. Furthermore, Wales was placed in the bottom quartile of the 42 countries and had the lowest levels of physical activity in 11-15 year olds in the UK (Inchley et al., 2016). International comparisons for TV watching ranked Wales in the top 5 for the proportion of children who watched 2 or more hours of TV on weekdays (Inchley et al., 2016).

Over the past 10 years, a significant effort has been made in Wales to influence children's physical activity and sport participation through programmes such as "Dragon Sport" for primary and " $5 \times 60$ " programmes for secondary age children. These programmes have been developed, implemented and evaluated and found evidence that children's extracurricular participation had increased and the range of activities that schools offered had broadened (Sport Wales, n.d.-b, 2011). Moreover, Sport Wales in collaboration with Public Health Wales and the education sector have urged Government to give physical literacy the same emphasis in the school curriculum as literacy and numeracy. To a degree, these have had an effect where the number of children hooked on sport has increased steadily since 2011 although inequalities still exist.

For outdoor and active play, Wales has an influential national government organisation that advocates for play ("Play Wales - Chwarae Cymru," n.d.). Wales was one of the first countries to integrate article 31 of the UNCRC of the child's right to play into policy ("United Nations Convention on the Rights of Children - Rights Children in Wales," n.d.), through its Children and Families (Wales) Measure 2010 
(Welsh Government, 2012). Moreover Play Wales has been referred to as "a highly focused, policy-oriented NGO” (Wales UNCRC Monitoring Group, 2015). Further, the grading for outdoor and active play was encouraging compared to other countries (Tremblay et al., 2014).

Walking and cycling have also received a policy boost in Wales. In 2013 the Welsh Government successfully introduced the 'Active Travel Act' onto its statute ("Active Travel (Wales) Act 2013," 2013). The increasing problem of children's sedentary time is worrying and we have recognised this in the shift of D to D-. Community and the built environment also decreased from a B in the 2014 Report Card to a C, although this was influenced by higher quality data that provided better insight into this indicator. Similar reasons explain the increase in grade given to family and peer influence that achieved a D+ compared to D in 2014. Wales has a vibrant physical education programme that is well resourced and links to community sport that are also well established, and also has a successful Healthy Schools Network that includes physical activity in its delivery portfolio (Public Health Wales, n.d.). Similar to a number of developed countries (Tremblay et al., 2014), school achieved the highest grade of all the indicators (B).

\subsubsection{Research Gaps}

The AHK Wales Report Card is produced every 2 years. There were significant gaps unveiled during the production of the AHK Wales 2014 Report Card and whilst many of these still remain, the AHK Wales 2016 process was built on a more complete data platform and a more robust approach by a more experienced RWG. The School Sport Survey (Sport Wales, 2015c) captured data on over 115,000 children in $61 \%$ of primary and $87 \%$ of secondary schools in Wales, building on data from 110,000 children 2 years earlier. The HBSC international report was published in 2016 (Inchley et al., 2016) and allowed detailed analysis of the 2013/14 data (Welsh Government, 2015a) permitting trends to be analysed and also data from Wales to be compared with other countries that used the same methodology (Inchley et al., 2016). On the other hand, there are currently no large-scale studies where physical activity or sedentary time have been measured objectively and inconsistency also exists across tools used to generate data on the 10 quality indicators. These issues are being partly addressed through the development of national indicators for sedentary time, physical activity and physical literacy by a Wales physical activity measurement and indicators group. The RWG also assigned a grade of 'inconclusive' to the physical literacy indicator. Sport Wales and Swansea University have developed an objective measure of phys- 
ical competency (Dragon Challenge) that will provide nationally representative data for use in future Report Cards.

One of the main challenges for the RWG was that most of the data available on children's physical activity and related behaviours were not aligned to quality indicators included in the Report Card. Further, the more interpretive indicators such as School, Family and Peer Influence, and National Government Policy, Strategies and Investments, and Community and the Built Environment required more synthesis of different levels and quality of data and expert opinion.

More recently, the University of South Australia used a Delphi process with two independent panels of international physical activity experts to produce 29 research priorities for the next decade (Gillis et al., 2013). The 3 top research priorities were (i) effective and sustainable interventions to affect long-term increases in children's physical activity behaviour; (ii) the influence of policy and environmental changes on physical activity and sedentary behaviour; and (iii) prospective, longitudinal studies of the independent effects of physical activity and sedentary behaviour on health (Gillis et al., 2013). Wales should seek to address these research priorities. Future Report Cards will note whether these issues have been addressed. In the meantime this Report Card will serve as an advocacy tool to promote physical activity research in Wales and to generate a national evidence base to underpin future AHK Wales Report Cards.

\subsubsection{Recommendations to improve the grades}

Policy makers, practitioners and educators should use the results of the AHK Wales 2016 Report Card to inform their decision processes. This is the second AHK Wales Report Card and, unlike more established cards, Wales is less familiar with using the results to influence factors affecting children's activity. Physical activity promotion in Wales has received criticism for lacking leadership and joined up approaches across Government portfolios (Wales Audit Office, 2007). This has resulted in fractures in strategy and delivery from national to local levels. Moreover, data provides the evidence to support the lack of impact on physical activity in Wales over the past decade (Welsh Government, 2015a, 2015c). Wales requires a clearly articulated ambition, and this ambition requires an unbroken chain of policy-strategy-delivery-review to translate these factors into positive physical activity outcomes. Nonetheless the RWG and stakeholders fully expect to build momentum behind children's physical activity research and promotion as the Report Card becomes more established and informa- 
tive. The RWG is following the lead given by Canada to provide evidence-guided recommendations to identify where investments of effort and resources can best influence the physical activity levels of children and youth (see for example, the dissemination, implementation, evaluation procedures for the Canadian 24- hour Movement Guidelines) (Tremblay, Carson, Chaput, et al., 2016). We are concerned that we have some of the lowest levels of physical activity and highest levels of sedentary behaviour globally (Inchley et al., 2016; Welsh Government, 2015a), and we will use these data to communicate and advocate approaches to improve this situation. Our overarching aim this year is to "turn the tide on physical inactivity" and make daily physical activity the social norm.

\subsubsection{Future Directions}

The Welsh Government has appointed a National Programme Director for Physical Activity to work across cabinet portfolios and we hope that this will have an effect on physical activity at all levels including children. The "Getting Wales Moving" strategy will be launched in the Summer of 2016 and prompt Welsh Government to develop a coordinated action plan based on the recommendations to resource an implementation and evaluation plan. The 'Transforming Public Health Strategy,' 'Communities First' and 'Wellbeing of Future Generations' (Wales) Acts have the potential to correct some of the fragmented approaches to promoting uptake of physical activity in the past.

The 2016 Global Summit on the Physical Activity of Children and the second meeting of the Active Healthy Kids Global Alliance provide valuable opportunities to learn from other countries that have a more established Report Card process or are in the early years of their respective Report Cards. In the future, AHK Wales will consult with researchers, knowledge users, and key stakeholders to re-examine the Report Card framework, indicators, and processes. This consultation will start with the AHK Wales 2016 Report Card launch and dissemination event on 25th July 2016 supported by an informative independent web site (www.activehealthykidswales.co.uk). Our future plans are to improve the current state of physical activity research and knowledge needs in order to enhance policies, programmes, practices, products, communications and investments to increase physical activity of children and youth in Wales.

\subsection{Conclusion}

The AHK Wales 2016 Report Card results demonstrate a need for focussed action and investment in promoting children's physical activity and reducing inactivity, seden- 
tary time and their related behaviours. There has been significant investment at policy structure and delivery levels since 2007 yet these have had little effect on children's physical activity levels which remain one of the lowest out of all countries surveyed in the HBSC report 2016 (Inchley et al., 2016). On the other hand Wales has placed active transport on its statute, has a strong track record in children's right to play and is changing the school curriculum to include a significant pillar on well-being. Furthermore inter-sectoral collaboration has helped promote children's physical literacy and activity and include them as indicators in future national surveys. This Report Card urges greater investment in children's physical activity so that future generations receive the health and developmental benefits of physical activity and reduce the spiralling social and economic burden of inactivity.

\subsection{Summary: Study 1}

This is the second AHK Wales Report Card. The 2016 version consolidates and translates research related to physical activity among children and youth in Wales and aims to raise the awareness of children's engagement in physical activity and sedentary behaviours.

Ten physical activity indicators were graded using the Active Healthy Kids - Canada Report Card methodology involving a synthesis and expert consensus of the best available evidence. Grades were assigned as follows; overall physical activity levels, D+; organized sport participation, C; active and outdoor play, C; active transportation, C; sedentary behaviours, D-; physical literacy, INC; family and peer influences, D+; school, B; community and the built environment, C; and national government policy, strategies and investments, B-.

Despite the existence of sound policies, programmes, and infrastructure, physical activity levels of children and youth in Wales are one of the lowest and sedentary behaviour highest globally. From the 2014 Report Card, family and peer influences grade has improved from $\mathrm{D}$ to $\mathrm{D}+$, whereas community and the built environment have dropped from $\mathrm{B}$ to $\mathrm{C}$. These results indicate that a concerted effort is required to increase physical activity and decrease sedentary time in children and young people in Wales. 


\section{Thesis Map}

Study

Outcomes

\begin{tabular}{|c|c|c|c|}
\hline \multirow[t]{2}{*}{1} & \multirow[t]{2}{*}{$\begin{array}{l}\text { Results from the Active Healthy } \\
\text { Kids Wales } 2016 \text { Report Card on } \\
\text { Physical Activity for Children and } \\
\text { Youth }\end{array}$} & Aim & $\begin{array}{l}\text { To consolidate and translate research related } \\
\text { to physical activity among children and } \\
\text { youth in Wales and to synthesise national } \\
\text { surveillance data from several surveys in } \\
\text { order to assign grades to physical } \\
\text { activity/inactivity behaviours and influences } \\
\text { indicators, as well as to examine trends or } \\
\text { inequalities in the data. }\end{array}$ \\
\hline & & $\begin{array}{c}\text { Key } \\
\text { Findings }\end{array}$ & $\begin{array}{l}\text { Grades were assigned as } \\
\text { follows; overall physical } \\
\text { activity levels, D+; organized } \\
\text { sport participation, C; active and } \\
\text { outdoor play, C; active } \\
\text { transportation, C; sedentary } \\
\text { behaviours, D-; physical } \\
\text { literacy, INC; family and peer } \\
\text { influences, D+; school, B; } \\
\text { community and the built } \\
\text { environment, C; and national } \\
\text { government policy, strategies } \\
\text { and investments, B-. }\end{array}$ \\
\hline \multirow[t]{2}{*}{2} & $\begin{array}{l}\text { Ten-year Secular } \\
\text { Changes in Selected } \\
\text { Health and Fitness } \\
\text { Parameters of 10-11 } \\
\text { Years Old Swansea } \\
\text { School Children - 2003- } \\
2013\end{array}$ & $\operatorname{Aim}$ & $\begin{array}{l}\text { To highlight the secular changes } \\
\text { in selected health and fitness } \\
\text { measures (body mass index, } \\
\text { prevalence of overweight and } \\
\text { obesity, and grip strength) in } \\
10-11 \text { year old Swansea school } \\
\text { children, using data obtained in } \\
2003 \text { and } 2013 \text {. }\end{array}$ \\
\hline & & Key Findings & \\
\hline 3 & $\begin{array}{l}\text { A Cross-Sectional Study on the } \\
\text { Socio-economic and Sex } \\
\text { Differences in Health-Related } \\
\text { Fitness Measures in School } \\
\text { Children }\end{array}$ & $\begin{array}{c}\text { Aim } \\
\text { Key Findings }\end{array}$ & \\
\hline 4 & $\begin{array}{l}\text { A Dynamic Assessment of } \\
\text { Children's Physical Competence: } \\
\text { The Dragon Challenge }\end{array}$ & $\begin{array}{c}\text { Aim } \\
\text { Key Findings }\end{array}$ & \\
\hline 5 & $\begin{array}{l}\text { Youth Physical Competence } \\
\text { Promotion Model: A Quantitative } \\
\text { Investigation into Modifiable } \\
\text { Factors }\end{array}$ & $\begin{array}{c}\text { Aim } \\
\text { Key Findings }\end{array}$ & \\
\hline
\end{tabular}




\section{Study 2}

\subsection{Ten-year Secular Changes in Selected Health and Fitness Pa- rameters of 10-11 Years Old Swansea School Children - 2003 $-2013$}

* This chapter is a published manuscript:

Tyler, R., Mackintosh, K., Palmer, A., Jones, A., \& Stratton, G. (2015). Ten-Year Secular Changes in Selected Health and Fitness Parameters of 10-11 Years Old Swansea School Children - 2003-2013. Advances in Obesity, Weight Management \& Control, 3(5), 8-13. https://doi.org/10.15406/aowmc.2015.03.00072

\subsection{Introduction}

In school-aged children, physical activity is associated with numerous physiological and psychosocial health benefits (Department of Health, 2011). In order to achieve these associated health benefits, current government recommends that children engage in moderate to vigorous intensity physical activity for at least 60 minutes every day (Department of Health, 2011). When these levels are attained, physical activity contributes to the prevention of childhood obesity (Guinhouya, 2012) and low physical fitness levels (Janssen \& Leblanc, 2010).

Children classified as overweight or obese are at risk of developing a number of obesity-related conditions (Reilly et al., 2003), including type 2 diabetes (Haines et al., 2007), metabolic and cardiovascular complications (Cote et al., 2013; L'AllemandJander, 2010), and mental health problems (Griffiths et al., 2010). Globally, the prevalence of overweight and obesity has more than doubled between 1980 and 2014 (World Health Organization, 2015). The number of children in Wales classified as overweight and obese represents a serious public health problem, with Wales being reported to have the highest rates of childhood obesity in the United Kingdom (National Obesity Observatory, 2015). However, recent reports on the prevalence of overweight and obesity in children in European countries, including England, indicate that the rise in the prevalence has plateaued or even, in some countries, has slightly decreased (Boddy et al., 2009; Boddy, Hackett, et al., 2010; Olds et al., 2011; Stratton et al., 2007; van Jaarsveld \& Gulliford, 2015). It is well documented that childhood obesity continues into adolescence and adulthood (Park et al., 2012); therefore it is important to identify the prevalence of childhood obesity to indicate not only current but also future health. 
Low levels of physical fitness in children are also linked with similar health-related outcomes as childhood obesity, including obesity itself (McMurray \& Andersen, 2010; Parfitt, Pavey, \& Rowlands, 2009; Ruiz, Castro-Piñero, et al., 2009). Levels of physical fitness in children have declined globally, as well as in the United Kingdom, in recent years (Boddy et al., 2012; Tomkinson \& Olds, 2007; Tremblay, Shields, et al., 2010). The majority of studies relating to physical fitness report on cardiorespiratory fitness (Boddy et al., 2012; Ortega et al., 2008; Tomkinson \& Olds, 2007; Tremblay, Shields, et al., 2010), however research has highlighted the need to assess the muscular strength component of physical fitness given its relationship with health outcomes and all-cause mortality (Cohen et al., 2011; De Miguel-Etayo et al., 2014; MolinerUrdiales et al., 2010; Ruiz, Castro-Piñero, et al., 2009; Tremblay, Shields, et al., 2010). Whilst there are numerous measures of strength, grip strength has been shown to be a good indicator for total muscular strength (Wind et al., 2010). Specifically, studies on grip strength have revealed its direct associations with chronic diseases, multi-morbidity and premature mortality (Griffiths et al., 2010; Metter et al., 2002; Ruiz, Castro-Piñero, et al., 2009; Volaklis et al., 2015). It is therefore postulated that grip strength should be measured in children in order to predict changes in overall strength, future health and mortality.

Although there is large evidence base that physical activity levels among children in Wales are low (Welsh Government, 2015c), there is a paucity of research monitoring the trends relating to obesity and physical fitness of children in Wales. Identifying changes in health and fitness levels among school children could indicate the need for focused services and interventions. Therefore, the aim of this study was to highlight the secular changes in selected health and fitness measures (body mass index, prevalence of overweight and obesity, and grip strength) in 10-11 year old Swansea school children, using data obtained in 2003 and 2013.

\subsection{Methods}

The current study uses data measured in 2003 during a Crucial Crew Day, and in 2013 during the Swan-Linx programme, both of which took place in Swansea, Wales. All testing procedures followed those highlighted in the EuroFit Battery (Council of Europe, 1993), and SportsLinx project (Taylor et al., 2004). The Swan-Linx programme has ongoing University ethical approval for its procedures and measures. The programme is a continuation of the SportsLinx project which has acceptable test/retest reliability for its field-based testing (Boddy, Stratton, \& Hackett, 2010). 


\subsubsection{Participants and Settings}

The current study uses data on children that were invited to take part in this study in both 2003 and 2013 from schools situated in the same electoral wards (areas) of Swansea. The children were all 10-11 years old (Year 6) at the time of testing, with a similar distribution of boys and girls at both testing periods. The schools were matched in terms of demographic status.

Data collected in 2003 were measured by staff and trained researchers from the Sport and Exercise Science department at Gower College. Participants were 10-11 year old children (Year 6) from primary schools situated across Swansea. The primary schools were invited to attend a Swansea Primary School Crucial Crew day, during which, the school children participated in health and fitness measures. Similarly, the 2013 data was obtained during the Swan-Linx programme's fitness fun days. Primary school children aged 9-11 year old (Year 5 and 6) from across Swansea were invited to take part in the programme, which was delivered by University staff and trained Active Young People officers from City and County of Swansea. The present study only uses data from the participants aged 10-11 years old (Year 6).

Out of all of the schools that took part in the events, data from thirteen schools in 2003 and thirteen schools in 2013 are used in this study. Eight schools from each of the 2003 and 2013 data were selected as these schools participated in both years of testing. From these eight schools, 258 children were measured in 2003 and 214 children were measured in 2013. An additional five schools from the 2003 data and five schools from the 2013 data were matched based on their demography using the Welsh Index of Multiple Deprivation (WIMD) scores. From these schools, 254 children were measured in 2003 and 200 children were measured in 2013. In total, 512 participants ( $n=230$ boys, $n=282$ girls) in 2003 and 414 participants ( $n=198$ boys, $\mathrm{n}=216$ girls) in 2013 were measured.

\subsubsection{Instruments and Procedures}

Stature was measured to the nearest $0.001 \mathrm{~m}$ and body mass was measured to the nearest $0.1 \mathrm{~kg}$ using a portable height stadiometer (Seca 213 portable stadiometer, Hamburg, Germany) and portable weighing scales (Seca 876, Hamburg, Germany), respectively. Techniques adhered to the Kinanthropometry and Exercise Physiology Manual (Eston \& Reilly, 2009). Body Mass Index (BMI) was calculated using these measures $\left(\mathrm{BMI}=\right.$ body mass $(\mathrm{kg}) /$ stature $\left.^{2}\left(\mathrm{~m}^{2}\right)\right)$. Hand-grip strength was measured 
as an indicator of overall muscular strength, using hand dynamometers (Takei Corp Ltd., Tokyo, Japan), giving a measure of the participant's strength in kilograms. Dominant hand readings were used in this study, as this reading was available in both 2003 and 2013 data.

\subsubsection{Design and Analysis}

Body Mass Index was used to calculate body mass index standard deviation scores (BMI z-scores), standardised for age and sex, using the 1990 British Growth BMI reference standard (Cole et al., 1995). Decimal age of participants was not available in the 2003 data, so estimated average age was calculated (average age of school children starting Year $6+$ date of testing) and used to standardise BMI to a point. The prevalence of unhealthy weight in terms of overweight and obese, were calculated using age and sex specific BMI cut-off points (Cole et al., 2000). Descriptive statistical analysis, mean and standard deviation (mean $\pm \mathrm{SD}$ ), were initially performed on all measures. A two-way Analysis of Variance (ANOVA) was conducted to examine if there was a statistically significant difference in BMI z-score by sex and year of testing. A two-way Analysis of Covariance (ANCOVA) was used to investigate if there were differences on grip strength by sex and year of testing, while controlling for BMI $\mathrm{z}$-score. BMI z-score was chosen as a covariate due to the known positive correlation of BMI and grip strength in both boys and girls during childhood (Milliken, Faigenbaum, Loud, \& Westcott, 2008). Statistical analysis was completed using SPSS, version 22 (IBM SPSS Statistics Inc., Chicago, IL, USA), with a significance level of 0.05 . If an interaction effect was present, a Bonferroni adjustment was applied for simple main effect analysis of year on each level of sex.

\subsection{Results}

Mean age for participants were 11.33 years and 10.99 years \pm 0.45 in 2003 and 2013, respectively. Mean stature, body mass and BMI z-scores for boys and girls in 2003 and 2013 are presented in Table 3. 
Table 3. Anthropometric measures (mean \pm SD) for boys and girls in 2003 and 2013

\begin{tabular}{|c|c|c|c|c|c|c|}
\hline \multirow{3}{*}{$\begin{array}{l}\text { Anthropometric } \\
\underline{\text { Measures }}\end{array}$} & \multicolumn{3}{|l|}{2003} & \multicolumn{3}{|l|}{2013} \\
\hline & Boys & Girls & All & Boys & Girls & All \\
\hline & \multicolumn{3}{|c|}{ Mean $\pm S D$} & \multicolumn{3}{|c|}{ Mean $\pm S D$} \\
\hline \multirow{2}{*}{ Stature $(\mathrm{m})$} & 1.42 & 1.44 & 1.43 & 1.43 & 1.45 & 1.44 \\
\hline & \pm 0.07 & \pm 0.07 & \pm 0.07 & \pm 0.07 & \pm 0.08 & \pm 0.07 \\
\hline \multirow{2}{*}{ Body Mass (kg) } & 40.2 & 41.3 & 40.8 & 38.2 & 40.8 & 39.5 \\
\hline & \pm 9.9 & \pm 9.1 & \pm 9.5 & \pm 9.1 & \pm 10.4 & \pm 9.9 \\
\hline \multirow{2}{*}{ BMI z-score } & 0.80 & 0.58 & 0.69 & 0.40 & 0.41 & 0.41 \\
\hline & \pm 1.24 & \pm 1.16 & \pm 1.20 & \pm 1.32 & \pm 1.23 & \\
\hline
\end{tabular}

Table Abbreviations: BMI z-score - Body Mass Index Standard Deviation Score

\subsubsection{BMI z-scores}

Mean BMI z-scores for boys and girls in 2003 and 2013 are presented in Figure 3. The interaction effect between sex and year on BMI z-scores was not statistically significant $\left(\mathrm{F}_{(1,890)}=1.790, \mathrm{p}=0.18\right)$. Thus, main effects analysis was conducted and displayed a significant main effect of year on BMI z-scores $\left(\mathrm{F}_{(1,890)}=11.490, \mathrm{p}=\right.$ $0.001)$ and a non-significant main effect of sex on BMI z-scores $\left(\mathrm{F}_{(1,890)}=1.476, \mathrm{p}=\right.$ $0.225)$, demonstrating that mean BMI z-scores decreased significantly for both boys and girls between 2003 and 2013. 


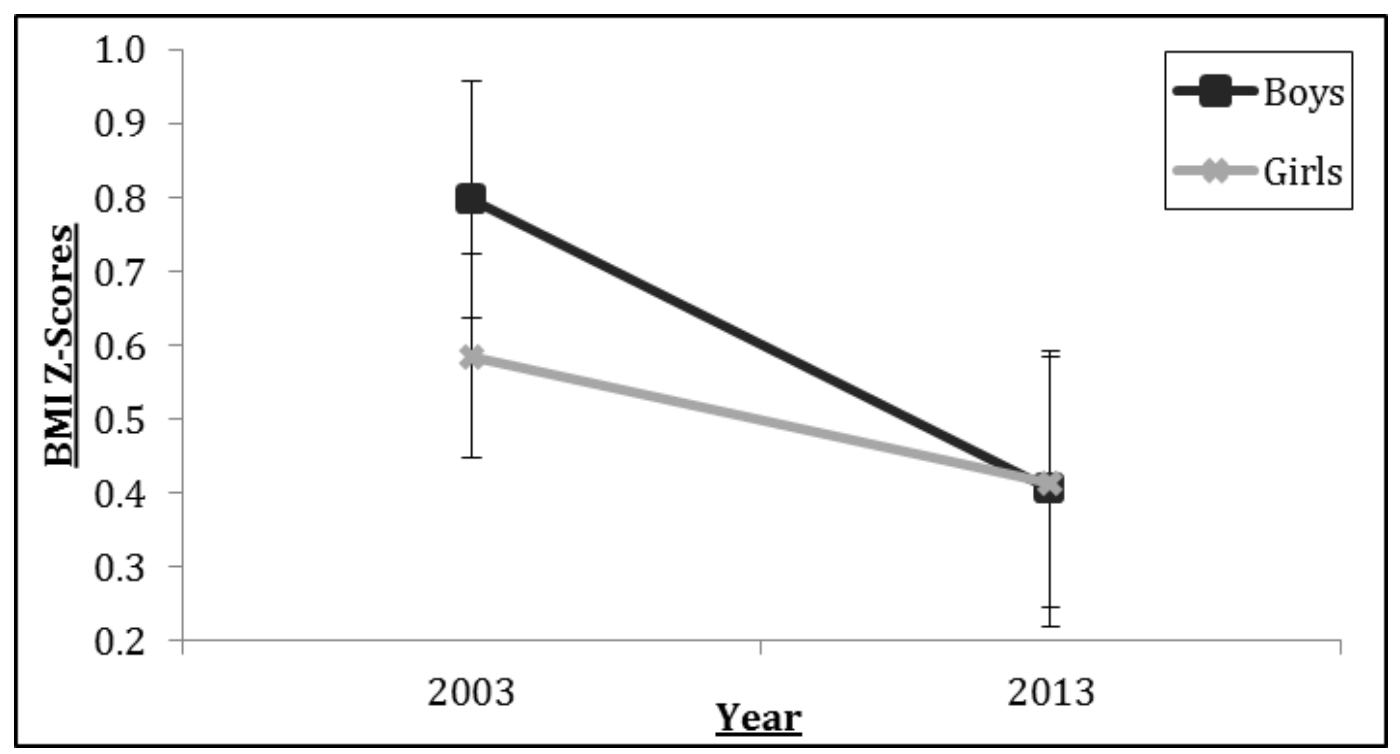

Figure 3. Mean BMI z-scores for boys and girls in 2003 and 2013, with 95\% confidence intervals.

\subsubsection{Prevalence of overweight and obesity}

The proportion of participants classified as overweight or obese in 2003 and 2013, using age and sex specific cut-off points for BMI z-scores (Cole et al., 2000), are presented in Table 4. Prevalence of overweight and obesity in participants shows a decrease from 2003 to 2013 for both boys and girls.

Table 4. Proportion of boys and girls in 2003 and 2013 classed as unhealthy weight and percentage difference between 2003 and 2013

\begin{tabular}{|l|l|l|l|l|l|l|l|l|l|}
\hline \multirow{2}{*}{$\begin{array}{l}\text { Prevalence of } \\
\text { unhealthy weight }\end{array}$} & \multicolumn{2}{|l|}{2003} & \multicolumn{2}{l|}{2013} & \multicolumn{3}{l|}{ Percentage Difference } \\
\cline { 2 - 11 } & Boys & Girls & All & Boys & Girls & All & Boys & Girls & All \\
\hline Overweight (\%) & 24.8 & 28.3 & 26.7 & 19.2 & 23.8 & 21.5 & -5.6 & -4.5 & -5.2 \\
\hline Obese (\%) & 7.0 & 5.6 & 6.2 & 4.7 & 5.9 & 5.3 & -2.3 & 0.3 & -0.9 \\
\hline Overweight or Obese (\%) & 31.7 & 33.8 & 32.9 & 23.8 & 29.7 & 26.8 & -7.9 & -4.1 & -6.1 \\
\hline
\end{tabular}

\subsubsection{Grip strength}

Mean grip strength for boys and girls in 2003 and 2013 are presented in Figure 4. Grip Strength was log transformed for statistical analysis, and then back transformed for presentation purposes. There was homogeneity of regression slopes, as the interaction term between Grip Strength (independent variable) and BMI z-scores (covariate) was not significant $(\mathrm{p}=0.126)$. Therefore, BMI z-scores were included as a covariate. 
Controlling for BMI z-scores, there was a statistically significant two-way interaction between sex and year $\left(\mathrm{F}_{(1,886)}=8.22, \mathrm{p}=0.004\right)$. Simple main effects analysis showed that boys in 2003 had significantly higher grip strength than boys in $2013\left(\mathrm{~F}_{(1,886)}\right.$ $=12.74, \mathrm{p}<0.001)$. However, there was no significant difference in grip strength between girls in 2003 and $2013\left(\mathrm{~F}_{(1,886)}=0.16, \mathrm{p}=0.69\right)$.

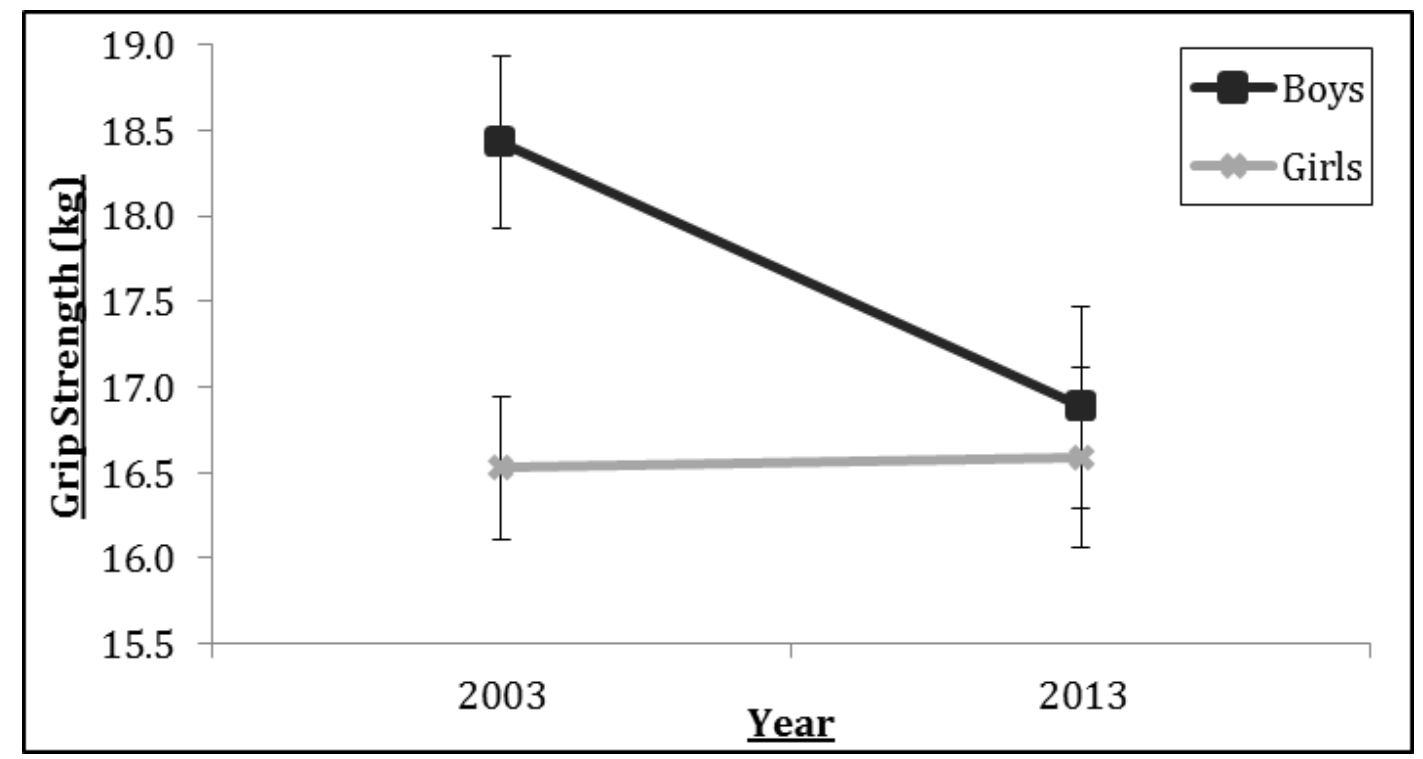

Figure 4. Mean grip strength for boys and girls in 2003 and 2013, with 95\% confidence intervals.

\subsection{Discussion}

The purpose of this study was to report secular changes in selected health and fitness measures, in 10-11 year old children between 2003 and 2013. Our main findings show that BMI z-scores decreased significantly in all children and grip strength significantly decreased in boys. To our knowledge these are the first 10-year secular changes in health and fitness measures reported on school-aged children in Wales.

\subsubsection{BMI and prevalence of overweight and obesity}

Body Mass Index has been shown to represent a practical method of assessing overweight and obesity in many large-scale paediatric studies (Boddy et al., 2009; Boddy, Hackett, et al., 2010; Olds et al., 2011; Stratton et al., 2007; van Jaarsveld \& Gulliford, 2015). In contrast with some reports that BMI and prevalence of overweight and obesity is still slowly rising or reaching a plateau (Boddy et al., 2009; Stratton et al., 2007; van Jaarsveld \& Gulliford, 2015), the current study shows a significant mean decline in BMI z-scores from 2003 to 2013 for both boys and girls, as well as a decline 
in prevalence of overweight and obesity in boys and girls, from $31.7 \%$ to $23.8 \%$ and $33.8 \%$ to $29.7 \%$, respectively. These findings are congruent with the slight decrease in prevalence shown in girls of similar age in a study conducted in England (Boddy, Hackett, et al., 2010), and also the decreases in BMI and prevalence in both boys and girls seen in other European countries (Olds et al., 2011). These results show promise for targeting childhood obesity in Wales and may therefore show some optimism for the future. It should be noted, however, that the prevalence of overweight and obesity recorded in 2013 is still high, with an average of one in four boys and girls being overweight or obese. Therefore, a substantial number of Swansea school children are still at risk for obesity-related health outcomes, and so further decreases in the prevalence should continue to be an aim.

\subsubsection{Muscular strength}

In the current study, mean grip strength significantly decreased for boys between 2003 and 2013 (18.43kg to $16.88 \mathrm{~kg}$ ), but there was no significant change for girls $(16.53 \mathrm{~kg}$ to $16.59 \mathrm{~kg}$ ). These findings, which cannot be explained by differences in BMI zscore, are likely related to changes in patterns of physical activity, which has been shown to be associated with muscular strength (Paalanne et al., 2009).

We note that in many past studies, boys and girls both display the same trends in grip strength changes over time. A study on Canadian children (Tremblay, Shields, et al., 2010), comparing data on 8-10 year olds between 1981 and 2007-2009, and a study on Spanish adolescents (Moliner-Urdiales et al., 2010), between 2001/02 and 2006/07, both showed declines in grip strength for both boys and girls. Further, a study in England, on 10-year old English children, also found a decline in grip strength for boys and girls over a 10-year secular trend from 1998 to 2008 (Cohen et al., 2011). The findings of the current study support these trends in terms of boys showing a decrease in grip strength, but, in contrast with the previous studies, grip strength for girls in the current study did not significantly change. The decrease in participation in extracurricular sports by year 3-6 school children in Mid \& West Wales (which includes Swansea), from $85.3 \%$ in 2002 (85.0\% in 2004) to $81.5 \%$ in 2013, reported in the School Sport Survey (Sport Wales, 2015c), may provide a possible explanation behind this inconsistency in the changes in grip strength between boys and girls. Ara et al. (2007), found that grip strength was significantly greater in boys who took part in extracurricular sporting activities than in boys who did not take part in these activities; however, there was no difference in grip strength between girls who did and did not take part in extracurricular sporting activities. Thus, the decrease in extracurricular 
activity participation described by the School Sports Survey may have resulted in a greater effect on grip strength performance in boys than it did in girls. Nonetheless, the decrease highlighted in grip strength in boys in the current study may mean that the boys may be at greater risk of future health-related illnesses (McMurray \& Andersen, 2010; Parfitt et al., 2009; Ruiz, Castro-Piñero, et al., 2009).

\subsubsection{Muscular strength and BMI}

Previous studies have shown a relationship between fat-free mass (muscle mass) and grip strength in adults (Lebrun, van der Schouw, de Jong, Grobbee, \& Lamberts, 2006; Yoon et al., 2009). Therefore, the decrease in grip strength in boys found in our study may be a result of a decrease in fat-free mass. We note that this may also provide a reason for the decline in BMI for boys, rather than the alternative notion of a decrease in excess adiposity (fat mass) (Field et al., 2003). Moreover, since there was no change in grip strength but there was a decrease in BMI for the girls in this study, then, using the same reasoning, this may indicate that there has been a decrease in excess adiposity in girls in this study. However, these conjectures cannot be concluded, and further investigation using more direct measures of adiposity and fat-free mass are required.

\subsubsection{Limitations}

There were some limitations to this study. Due to not having specific ages for the children in the 2003 data, BMI was corrected to a single age (the average age of children in Year 6 at the time of testing), which limits the adjustment for age to a single point, and may result in a slight over or under prediction of the average BMI z-score. Further, it is difficult to distinguish whether changes in BMI are a result of changes in levels of fat or fat-free mass have changed over the 10-year period. Future studies should aim to include a range of anthropometric assessments, including skinfolds and circumferences (Eston \& Reilly, 2009), or dual-energy X-ray absorptiometry (DEXA) (Lohman \& Going, 2006), to assess fat and fat-free mass and percentage body fat.

It should also be mentioned that deprivation, an important factor towards children's health and fitness, was not controlled for in the analysis of data, due to only having school postcodes rather than home postcodes available for the 2003 data, and using school postcodes has been shown to be a less effective way of controlling for deprivation as children may travel across postcodes to school (Dummer, Gibbon, Hackett, Stratton, \& Taylor, 2005). However, this should not be considered as a limitation to the study as all children lived in the same electoral wards and little has changed in the 
demographics of these wards between 2003 and 2013.

Finally, when interpreting the findings from the present study, it is important to note that only two datasets (2003 and 2013) were used to describe the changes over a tenyear period. This limits the ability to analyse year-on-year variations in the trend. There is a great need to continue to monitor trends to assess these year-on-year variations, as well as the effectiveness of any interventions working towards improving children's health and fitness. Moreover, continued monitoring could be useful in identifying whether these trends continue into older children once they have more control over their exercise and eating habits.

\subsection{Conclusion}

The current study is the first to report 10-year secular changes in health and fitness measures of schoolchildren in Wales. The study shows promising results in terms of BMI and prevalence of overweight and obesity. However, the study also shows that muscular strength, in boys in particular, should be a focus for future interventions regarding physical fitness, in addition to an area for further investigation. It is unclear whether the trends observed in the current study will continue or if the reductions seen in BMI and prevalence of overweight and obesity, as well as in grip strength in boys, are part of a year-on-year variation, with the children in 2013 being an abnormally low fluctuation in a year-on-year trend. Thus, there is a need for longitudinal data to show year-on-year trends in order to assess annual fluctuations.

\subsection{Summary: Study 2}

The aim of this chapter was to highlight the secular changes in selected health and fitness measures (body mass index, prevalence of overweight and obesity, and grip strength) in 10-11 year old Swansea school children, using data obtained in 2003 and 2013.

Accordingly, BMI z-scores decreased significantly $(\mathrm{p}=0.001)$ between 2003 and 2013 for both boys ( 0.80 to 0.40$)$ and girls $(0.58$ to 0.41$)$. Prevalence of overweight and obesity decreased for both boys and girls from $31.7 \%$ to $23.8 \%$ and $33.8 \%$ to $29.7 \%$, respectively. After BMI z-scores adjustment, grip strength decreased significantly for boys $(18.43 \mathrm{~kg}$ to $16.88 \mathrm{~kg}, \mathrm{p}<0.001)$, but not for girls $(16.53 \mathrm{~kg}$ to $16.59 \mathrm{~kg})$.

While this study shows encouraging results in terms of BMI z-scores and prevalence 
of overweight and obesity, further decreases in prevalence should continue to be an aim as the prevalence of overweight and obesity recorded in 2013 is still high. The study also shows that muscular strength, in boys in particular, should be a focus for future interventions regarding physical fitness. 


\section{Thesis Map}

\begin{tabular}{|c|c|c|c|}
\hline & Study & & Outcomes \\
\hline 1 & $\begin{array}{l}\text { Results from the Active Healthy } \\
\text { Kids Wales } 2016 \text { Report Card on } \\
\text { Physical Activity for Children and } \\
\text { Youth }\end{array}$ & Key Findings & $\begin{array}{l}\text { To consolidate and translate research related } \\
\text { to physical activity among children and } \\
\text { youth in Wales and to synthesise national } \\
\text { surveillance data from several surveys in } \\
\text { order to assign grades to physical } \\
\text { activity/inactivity behaviours and influences } \\
\text { indicators, as well as to examine trends or } \\
\text { inequalities in the data. } \\
\text { Grades were assigned as follows; overall } \\
\text { physical activity levels, D+; organized sport } \\
\text { participation, C; active and outdoor play, C; } \\
\text { active transportation, C; sedentary } \\
\text { behaviours, D-; physical literacy, INC; } \\
\text { family and peer influences, D+; school, B; } \\
\text { community and the built environment, C; } \\
\text { and national government policy, strategies } \\
\text { and investments, B-. }\end{array}$ \\
\hline \multirow[t]{2}{*}{2} & \multirow[t]{2}{*}{$\begin{array}{l}\text { Ten-year Secular Changes in } \\
\text { Selected Health and Fitness } \\
\text { Parameters of 10-11 Years Old } \\
\text { Swansea School Children - 2003- } \\
2013\end{array}$} & Aim & $\begin{array}{l}\text { To highlight the secular changes in selected } \\
\text { health and fitness measures (body mass } \\
\text { index, prevalence of overweight and obesity, } \\
\text { and grip strength) in 10-11 year old Swansea } \\
\text { school children, using data obtained in } 2003 \\
\text { and } 2013 \text {. }\end{array}$ \\
\hline & & $\begin{array}{c}\text { Key } \\
\text { Findings }\end{array}$ & $\begin{array}{l}\text { BMI z-scores, as well as } \\
\text { prevalence of overweight and } \\
\text { obesity, decreased significantly } \\
\text { between } 2003 \text { and } 2013 \text { for both } \\
\text { boys and girls. After BMI z- } \\
\text { scores adjustment, grip strength } \\
\text { decreased significantly for boys, } \\
\text { but not for girls. }\end{array}$ \\
\hline \multirow[t]{2}{*}{3} & $\begin{array}{l}\text { A Cross-Sectional Study } \\
\text { on the Socio-economic } \\
\text { and Sex Differences in } \\
\text { Health-Related Fitness } \\
\text { Measures in School } \\
\text { Children }\end{array}$ & Aim & $\begin{array}{l}\text { To investigate socio-economic } \\
\text { and sex differences in selected } \\
\text { health-related fitness measures } \\
\text { (body mass index, MSRT, grip } \\
\text { strength) in 9-12-year-old } \\
\text { children. }\end{array}$ \\
\hline & & Key Findings & \\
\hline 4 & $\begin{array}{l}\text { A Dynamic Assessment of } \\
\text { Children's Physical Competence: } \\
\text { The Dragon Challenge }\end{array}$ & $\begin{array}{c}\text { Aim } \\
\text { Key Findings }\end{array}$ & \\
\hline 5 & $\begin{array}{l}\text { Youth Physical Competence } \\
\text { Promotion Model: A Quantitative } \\
\text { Investigation into Modifiable } \\
\text { Factors }\end{array}$ & $\begin{array}{c}\text { Aim } \\
\text { Key Findings }\end{array}$ & \\
\hline
\end{tabular}




\section{Study 3}

\subsection{A Cross-Sectional Study on the Socio-economic and Sex Dif- ferences in Health-Related Fitness Measures in School Chil- dren}

* This chapter is under review for publication:

Tyler, R., Mackintosh, K. A., Spacey, H. L. \& Stratton, G. (2018). A Cross-Sectional Study on the Socio-economic and Sex Differences in Health-Related Fitness Measures in School Children. Journal of Sports Sciences (under review).

\subsection{Introduction}

The World Health Organization defines health inequalities as "the differences in health status or in the distribution of health determinants between different population groups" (World Health Organization, n.d.). Health is not equally spread across UK society, with children from deprived areas at higher risk of poor health (Public Health Wales Observatory, 2013; Wickham, Anwar, Barr, Law, \& Taylor-Robinson, 2016). Physical activity has been identified as key to enhancing numerous physiological, psychosocial and cognitive health benefits in children (Biddle \& Asare, 2011; Boreham \& McKay, 2011; Janssen \& Leblanc, 2010), while preventing emerging health risk factors (Andersen et al., 2011; Janssen \& Leblanc, 2010). For children and young people aged 5-18 years to receive the associated benefits of physical activity, the UK physical activity guidelines (Department of Health, 2011), recommend engagement in moderate to vigorous intensity activity for at least 60 minutes and up to several hours each day. In particular, vigorous intensity activities, including those that strengthen muscle and bone, should be incorporated at least three days per week (Department of Health, 2011). The physical activity levels of children and young people in Wales are low and lie in the bottom quartile of very high human development index countries (Aubert et al., 2018; Tremblay, Gonzalez, et al., 2016; Tyler et al., 2016). Moreover, in accord with health inequality research, physical activity levels have been reported to be higher in boys than girls (Tyler et al., 2016; Welsh Government, 2015a, 2017). However, whilst there is a range of physical activity levels, results display little difference in average levels between deprivation groups (Tyler et al., 2016; Welsh Government, 2015a, 2017).

Whilst physical activity levels and inequalities are well established, physical fitness has been shown to be a stronger indicator of health than physical activity (Andersen 
et al., 2011). Physical fitness includes a range components that are associated with health outcomes, such as cardiorespiratory fitness, muscular strength/fitness and body composition (Ruiz et al., 2011). In children, many studies have reported the strong associations between low cardiorespiratory fitness (McMurray \& Andersen, 2010; Ortega et al., 2008; Ruiz, Castro-Piero, et al., 2009), low muscular strength/fitness (Ortega et al., 2008; Ruiz, Castro-Piñero, et al., 2009; Volaklis et al., 2015) and high body mass index (Griffiths et al., 2010; L'Allemand-Jander, 2010; Ruiz, Castro-Piñero, et al., 2009), with metabolic and cardiovascular disease risk, mental health problems, reduced quality of life and all-cause mortality (Högström et al., 2015; Ruiz, CastroPiñero, et al., 2009; Volaklis et al., 2015). Therefore, identifying differences in fitness levels among school children could indicate the need for focused services and interventions to improve health inequalities.

Although some recent UK studies have considered health-related fitness measures and socio-economic status in statistical analyses (Brophy, Rees, Knox, Baker, \& Thomas, 2012; Charlton et al., 2014; Nevill, Duncan, Lahart, \& Sandercock, 2017; Noonan, Boddy, Knowles, \& Fairclough, 2016, 2017), there were inherent limitations. Specifically, the predominant focus was not comparing multiple fitness measures across socio-economic status, and the variation in health-related fitness variables included in these studies limits inter-study comparisons (Nevill et al., 2017; Noonan et al., 2016, 2017). Additionally, these studies neglected to include any measure of the muscular strength component of physical fitness (Brophy et al., 2012; Charlton et al., 2014; Nevill et al., 2017; Noonan et al., 2016, 2017). Research has purported the need to assess this fitness component given its individual relationship with health outcomes (Ortega et al., 2008; Ruiz, Castro-Piñero, et al., 2009; Volaklis et al., 2015), with grip strength, specifically, having direct associations with cardio-metabolic disease risk and early mortality (Ortega et al., 2008; Volaklis et al., 2015). Moreover, Brophy et al. (2012), and Charlton et al. (2014), used the number of children receiving free school meals and school's postcode as a proxy measure of socio-economic deprivation. However, using a solely income-based proxy for deprivation (i.e. free school meals) overlooks the multiple dimensions/domains of deprivation that effect children (Noble, Wright, Smith, \& Dibben, 2006), while using school postcode has been shown to lack effectiveness as children may travel across postcodes to school (Boddy, Hackett, et al., 2010). It is therefore postulated that levels of health-related fitness, particularly grip strength, should be measured across socio-economic status groups with use of the index of multiple deprivation to predict health inequalities in children. 
The aim of this study was to investigate socio-economic and sex differences in selected health-related fitness measures in 9-12-year-old children.

\subsection{Methods}

\subsubsection{Participants and Settings}

A sample of 3,407 children ( $49.3 \%$ boys; aged $10.5 \pm 0.6$ years) participated in the study. Data were collected on children as part of the Swan-Linx health, fitness and lifestyle programme (Sheldrick, Tyler, Mackintosh, \& Stratton, 2018; Tyler, Mackintosh, Brophy, et al., 2015; Tyler, Mackintosh, Palmer, Jones, \& Stratton, 2015), which includes selected measures from the SportsLinx project (Stratton et al., 2007; Taylor et al., 2004). Data were captured during Fitness Fun Days between 2013-2017, across 29 socio-demographically representative schools, in South Wales, UK. All measures were conducted in school sport halls or at Swansea University's Indoor Training Centre, by trained researchers, local community coaches and Active Young People officers. Written informed head teacher and parental consent, as well as child assent, were obtained prior to data collection. The Swan-Linx programme has institutional Research Ethics Committee approval (PG/2014/007; PG/2014/37) for its procedures and measures.

\subsubsection{Measures and Procedures}

\section{Health-related Fitness:}

Three components of health-related fitness were measured, namely cardiorespiratory fitness, muscular strength and body mass index, using standardised methods (Council of Europe, 1993; Sheldrick et al., 2018; Taylor et al., 2004; Tyler, Mackintosh, Palmer, et al., 2015).

20m Multistage Shuttle Run Test (20m MSRT): The 20m MRST, shown to be valid and reliable in similarly-aged children, assessed cardiorespiratory fitness (MayorgaVega, Aguilar-Soto, \& Viciana, 2015; Riddoch, 1990). To ensure a consistent pace and to give encouragement, a researcher completed the test with the participants. The total number of completed shuttles was recorded.

Handgrip Strength Test: The Handgrip Strength test was used to measure upper body muscular strength and provide an indication of overall muscular strength (Ortega et al., 2008; Wind et al., 2010). Using hand dynamometers [Takei Corp Ltd., Tokyo, 
Japan], each participant's grip strength was measured in kilograms.

Anthropometric Measures: All anthropometric measures were taken by the same trained researcher using standard techniques (Lohman, Roche, \& Martorell, 1988). Each participant's stature, sitting stature, and body mass were measured to the nearest $0.1 \mathrm{~cm}$ and $0.1 \mathrm{~kg}$, using a portable stadiometer [Seca 213 portable stadiometer, Seca Ltd, Birmingham, UK], a sitting stature stadiometer [Harpenden Sitting Height Table, Holtain Ltd, Pembrokeshire, UK], and electronic weighing scales [Seca 876, Seca Ltd, Birmingham, UK]. Body Mass Index (BMI) was calculated (BMI = body mass $(\mathrm{kg}) /$ stature $\left.^{2}\left(\mathrm{~m}^{2}\right)\right)$ as a proxy measure of body composition, and BMI z-scores were derived using the UK 1990 growth reference curves (Cole et al., 1995). Sex-specific maturation offset regression equations using interactions between body mass, stature, sitting stature, lower limb length (difference between stature and sitting stature) and decimal age, were used to predict each participant's age from peak height velocity (Mirwald et al., 2002). This calculation was used as a proxy measure of biological maturation.

\section{Deprivation:}

Welsh Index of Multiple Deprivation (WIMD): The Welsh Index of Multiple Deprivation (WIMD) is used as the official measure of deprivation in Wales (Welsh Government, n.d.), in which eight domains of deprivation (employment; health; income; housing; community safety; access to services; education and the environment) are considered to provide an area-based socio-economic measurement (Noble et al., 2006). WIMD ranks have been calculated for each of Wales' 1,909 Lower-Layer Super Output Areas (LSOA), with the most deprived LSOA ranking 1, and the least deprived ranking 1,909. Participants' home postcodes were used to calculate WIMD scores, which were then split into WIMD quintile one (WIMD 23-226), two (WIMD 227-710), three (WIMD 711-982), four (WIMD 983-1604) and five (WIMD 16051908), with $20 \%$ of participants in each group.

\subsubsection{Statistical Analyses}

Descriptive statistical analysis, mean and standard deviation (mean \pm SD), were initially performed on all measures. Statistical analysis was completed using SPSS, version 25 (IBM SPSS Statistics Inc., Chicago, IL, USA), with a significance level of 0.05. Participant results were included in each respective analysis if they had sufficient data for the variable concerned. Due to the nested nature of the data (i.e. participants nested within schools), intraclass correlation coefficients (ICC) were calculated prior 
to further analyses to determine the proportion of the total variability in BMI z-score, grip strength, and 20m MSRT that could be attributed to school. Subsequently, a twoway Analysis of Variance (ANOVA) was conducted to examine whether there was a statistically significant difference in BMI z-score by sex and WIMD quintiles. A two-way Analysis of Covariance (ANCOVA) was used to investigate if there were differences in grip strength by sex and WIMD quintiles, while adjusting for BMI z-score. BMI z-score was chosen as a covariate due to the known positive correlation of BMI and grip strength during childhood, irrespective of sex (Milliken et al., 2008). A second two-way ANCOVA examined the effect of sex and WIMD quintiles on shuttles achieved in the $20 \mathrm{~m}$ MSRT, after adjusting for maturation, given its potential confounding effect. The suitability of the covariates in each ANCOVA model was investigated by ensuring the homogeneity of regression slopes assumption was met, as determined by a comparison between the two-way ANCOVA model with and without interaction terms. If an interaction effect was present in the ANCOVA model, analysis of simple main effects of both WIMD quintiles and sex was performed, with statistical significance receiving a Bonferroni adjustment $(\mathrm{p}<0.05 \div$ number of simple main effects made), to correct for the testing of multiple simple main effects. In the case of a non-significant interaction effect, analyses of main effects were performed. If a significant simple main effect or significant main effect was present, pairwise comparisons were run with $\mathrm{p}$-values Bonferroni-adjusted ( $\mathrm{p}<0.05 \div$ number of comparisons). Hedges' $g$ was used as a measure of effect size for pairwise comparisons.

\subsection{Results}

The descriptive characteristics of the study participants are presented in Table 5. Intraclass correlation coefficients for BMI z-score $(\mathrm{ICC}=0.014)$, grip strength $(\mathrm{ICC}=$ 0.083 ), and 20m MSRT ( ICC $=0.032$ ) demonstrated little statistical dependency in the data, suggesting that the largest proportion of variance lies among participants. 
Table 5. Descriptive statistics (mean \pm SD) for Study 3 participants

\begin{tabular}{lccc}
\hline \hline Variables & Boys & Girls & All \\
\hline Age (years) & $10.6 \pm 0.6$ & $10.5 \pm 0.6$ & $10.5 \pm 0.6$ \\
Stature (cm) & $142.3 \pm 7.3$ & $142.8 \pm 7.9$ & $142.6 \pm 7.6$ \\
Sitting Stature (cm) & $74.4 \pm 4.1$ & $74.9 \pm 4.5$ & $74.6 \pm 4.4$ \\
Body Mass (kg) & $38.4 \pm 10.1$ & $39.7 \pm 10.3$ & $39.0 \pm 10.2$ \\
Lower Limb Length (cm) & $67.9 \pm 5.0$ & $68.0 \pm 5.1$ & $67.9 \pm 5.1$ \\
BMI (kg.m-2) & $18.7 \pm 3.7$ & $19.3 \pm 3.8$ & $19.0 \pm 3.8$ \\
BMI z-score & $0.6 \pm 1.3$ & $0.6 \pm 1.3$ & $0.6 \pm 1.3$ \\
Maturity Offset (years) & $-2.8 \pm 0.6$ & $-1.2 \pm 0.7$ & $-2.0 \pm 1.0$ \\
Deprivation Score (WIMD) & $858.7 \pm 598.6$ & $869.6 \pm 588.9$ & $864.2 \pm 593.7$ \\
20m MSFT Score (shuttles) & $35.0 \pm 18.3$ & $24.7 \pm 12.9$ & $29.8 \pm 16.6$ \\
Grip Strength (kg) & $16.9 \pm 4.1$ & $16.0 \pm 3.9$ & $16.4 \pm 4.0$ \\
\hline
\end{tabular}

The interaction effect between WIMD quintiles and sex on BMI z-scores was not statistically significant $\left(F_{(4,3318)}=1.240, \mathrm{p}=0.292\right)$. Means \pm SD for BMI z-scores, for boys and girls in WIMD quintile one to five are shown in Table 6. Main effects analysis displayed a significant main effect of WIMD quintiles on BMI z-scores $\left(F_{(4,3318)}=\right.$ $3.488, \mathrm{p}=0.008)$. Pairwise comparisons revealed a statistically significant difference of 0.215 (95\% CI, 0.12 to 0.419; $\mathrm{p}=0.029, g=0.170$ ) between mean BMI z-score in WIMD quintile 3 and WIMD quintile 5, but there were no other significant mean differences in BMI z-score between WIMD quintile groups. There was no significant main effect of sex on BMI z-scores $\left(F_{(4,3318)}=0.811, \mathrm{p}=0.368\right)$. 
Table 6. Mean \pm SD BMI z-scores by WIMD quintile (deprivation) groups

BMI z-scores

\begin{tabular}{|c|c|c|c|}
\hline \multirow[b]{2}{*}{ Quintiles } & \\
\hline & Boys & Girls & All \\
\hline $\begin{array}{l}\text { WIMD quintile } 1^{\diamond} \\
(\mathrm{n}=699,51.9 \% \text { boys })\end{array}$ & $0.60 \pm 1.34$ & $0.66 \pm 1.32$ & $0.63 \pm 1.33$ \\
\hline $\begin{array}{l}\text { WIMD quintile } 2 \\
\text { ( } \mathrm{n}=676,49.1 \% \text { boys) }\end{array}$ & $0.61 \pm 1.32$ & $0.66 \pm 1.36$ & $0.63 \pm 1.34$ \\
\hline $\begin{array}{l}\text { WIMD quintile } 3 \\
(\mathrm{n}=620,48.7 \% \text { boys })\end{array}$ & $0.78 \pm 1.37$ & $0.56 \pm 1.26$ & $0.67 \pm 1.32$ \\
\hline $\begin{array}{l}\text { WIMD quintile } 4 \\
(\mathrm{n}=671,48.0 \% \text { boys })\end{array}$ & $0.53 \pm 1.27$ & $0.47 \pm 1.30$ & $0.50 \pm 1.29$ \\
\hline $\begin{array}{l}\text { WIMD quintile } 5^{\dagger} \\
(\mathrm{n}=662,50.8 \% \text { boys })\end{array}$ & $0.48 \pm 1.21$ & $0.44 \pm 1.18$ & $0.46 \pm 1.19$ \\
\hline
\end{tabular}

Note: WIMD quintile $1^{\diamond}=$ most deprived, WIMD quintile $5^{\dagger}=$ least deprived

Given that there was homogeneity of regression slopes $\left(F_{(9,3281)}=1.066, \mathrm{p}=0.385\right)$, BMI z-scores were included as a covariate in the ANCOVA model for grip strength. Adjusted means \pm SE (by BMI z-score) for grip strength are shown in Table 7. There was no statistically significant two-way interaction between WIMD quintiles and sex on grip strength, while adjusting for BMI z-scores $\left(F_{(4,3290)}=0.696, \mathrm{p}=0.595\right)$. The main effect of sex was statistically significant $\left(F_{(4,3290)}=39.336, \mathrm{p}<0.001, \mathrm{~g}\right.$ $=0.205)$, with boys adjusted mean grip strength 0.823 (95\% CI, 0.565 to 1.080$) \mathrm{kg}$ higher than girls. There was also a statistically significant main effect of WIMD quintile on grip strength $\left(F_{(4,3290)}=3.919, \mathrm{p}=0.004\right)$. Pairwise comparisons revealed a statistically significant difference of 0.718 (95\% CI, 0.143 to $1.294 ; \mathrm{p}=0.005, \mathrm{~g}=$ $0.174) \mathrm{kg}$ between adjusted mean grip strength in WIMD quintile 1 and WIMD quintile 5, but no other significant differences in grip strength between WIMD quintiles were found. 
Table 7. Adjusted mean \pm SE grip strength (adjusted by BMI z-scores) by WIMD quintile (deprivation) groups

\begin{tabular}{|c|c|c|c|}
\hline \multirow[b]{2}{*}{ Quintiles } & \multicolumn{3}{|c|}{ Adjusted Mean Grip Strength (kg) } \\
\hline & Boys & Girls & All \\
\hline $\begin{array}{l}\text { WIMD quintile } 1^{\diamond} \\
\text { ( } \mathrm{n}=695,51.8 \% \text { boys) }\end{array}$ & $16.4 \pm 0.2$ & $16.0 \pm 0.2$ & $16.2 \pm 0.1$ \\
\hline $\begin{array}{l}\text { WIMD quintile } 2 \\
(\mathrm{n}=672,49.0 \% \text { boys })\end{array}$ & $16.7 \pm 0.2$ & $16.0 \pm 0.2$ & $16.4 \pm 0.2$ \\
\hline $\begin{array}{l}\text { WIMD quintile } 3 \\
\text { (n=616, } 48.5 \% \text { boys })\end{array}$ & $16.7 \pm 0.2$ & $15.7 \pm 0.2$ & $16.2 \pm 0.2$ \\
\hline $\begin{array}{l}\text { WIMD quintile } 4 \\
(\mathrm{n}=660,47.7 \% \text { boys })\end{array}$ & $17.0 \pm 0.2$ & $16.0 \pm 0.2$ & $16.5 \pm 0.2$ \\
\hline $\begin{array}{l}\text { WIMD quintile } 5^{\dagger} \\
\text { (n=658,51.1\% boys) }\end{array}$ & $17.4 \pm 0.2$ & $16.4 \pm 0.2$ & $16.9 \pm 0.2$ \\
\hline
\end{tabular}

Given that the interaction term between 20m MSRT and maturation was not significant $(\mathrm{p}>0.05)$, there was homogeneity of regression slopes and so maturation was included as a covariate in the ANCOVA model for 20m MSRT. Adjusted means $\pm \mathrm{SE}$ (by maturation) for 20m MSRT score are shown in Table 8 . There was a statistically significant interaction between WIMD quintiles and sex on 20m MSRT, while controlling for maturation $\left(F_{(4,3182)}=2.659, \mathrm{p}=0.031\right)$. Simple main effects revealed that sex was statistically significant in WIMD quintile group $1\left(F_{(1,3182)}=11.437, \mathrm{p}=\right.$ $0.001, g=0.319)$, group $2\left(F_{(1,3182)}=35.100, \mathrm{p}<0.001, g=0.534\right)$, group $3\left(F_{(1,3182)}\right.$ $=11.560, \mathrm{p}=0.001, g=0.297)$, group $4\left(F_{(1,3182)}=32.391, \mathrm{p}<0.001, g=0.475\right)$, and group $5\left(F_{(1,3182)}=42.138, \mathrm{p}<0.001, g=0.566\right)$, with boys achieving a significantly greater 20m MSRT score than girls in each WIMD quintile group. Moreover, simple main effects showed that WIMD quintile group was statistically significant in girls $\left(F_{(4,3182)}=13.793, \mathrm{p}<0.001\right)$, and boys $\left(F_{(4,3182)}=3.481, \mathrm{p}=0.008\right)$. For girls, WIMD quintile 1 had a significantly lower adjusted mean 20m MSRT score than WIMD quintile 5 (3.694 shuttles, $95 \% \mathrm{CI}, 0.230$ to $7.157 ; \mathrm{p}=0.028, g=0.292$ ), but there were no statistically significant differences between adjusted mean 20m MSRT scores when comparing all other WIMD quintiles. For boys, WIMD quintile 1 had a significantly lower adjusted mean 20m MSRT score than WIMD quintile 2 (4.380 shuttles, $95 \% \mathrm{CI}, 0.969$ to $7.792 ; \mathrm{p}=0.003, g=0.251)$, WIMD quintile 4 (6.544 
shuttles, 95\% CI, 3.121 to 9.967; $\mathrm{p}<0.001, g=0.379$ ), and WIMD quintile 5 (7.921 shuttles, 95\% CI, 4.529 to 11.313; p $<0.001, g=0.469$ ). Further, boys' adjusted mean 20m MSRT score in WIMD quintile 2 was significantly lower than WIMD quintile 5 (3.541 shuttles, 95\% CI, 0.066 to 7.016; $\mathrm{p}=0.042, g=0.200$ ). Finally, boys' WIMD quintile 3 had a significantly lower adjusted mean 20m MSRT score than both WIMD quintile 4 (4.307 shuttles, 95\% CI, 0.747 to 7.867; $\mathrm{p}=0.007, g=0.229$ ) and WIMD quintile 5 (5.684 shuttles, 95\% CI, 0.969 to $7.792 ; \mathrm{p}=0.003, g=0.310$ ). All other pairwise comparisons between quintiles for boys' adjusted mean 20m MSRT score were non-significant.

Table 8. Adjusted mean \pm SE 20m MSRT (adjusted for maturation) by WIMD quintile (deprivation) groups

Adjusted Mean 20m MSRT (shuttles)

\begin{tabular}{|c|c|c|c|}
\hline Quintiles & Boys & Girls & All \\
\hline $\begin{array}{l}\text { WIMD quintile } 1^{\diamond} \\
(\mathrm{n}=670,51.6 \% \text { boys })\end{array}$ & $29.0 \pm 0.9$ & $24.4 \pm 0.9$ & $26.7 \pm 0.6$ \\
\hline $\begin{array}{l}\text { WIMD quintile } 2 \\
(\mathrm{n}=635,49.4 \% \text { boys })\end{array}$ & $33.4 \pm 0.9$ & $25.1 \pm 0.9$ & $29.3 \pm 0.6$ \\
\hline $\begin{array}{l}\text { WIMD quintile } 3 \\
\text { ( } \mathrm{n}=605,48.8 \% \text { boys })\end{array}$ & $31.3 \pm 1.0$ & $26.5 \pm 0.9$ & $28.9 \pm 0.6$ \\
\hline $\begin{array}{l}\text { WIMD quintile } 4 \\
\text { ( } \mathrm{n}=646,48.0 \% \text { boys) }\end{array}$ & $35.6 \pm 0.9$ & $27.8 \pm 0.9$ & $31.7 \pm 0.6$ \\
\hline $\begin{array}{l}\text { WIMD quintile } 5^{\dagger} \\
(\mathrm{n}=637,50.4 \% \text { boys })\end{array}$ & $37.0 \pm 0.9$ & $28.1 \pm 0.9$ & $32.5 \pm 0.6$ \\
\hline
\end{tabular}

\subsection{Discussion}

The aim of this study was to investigate socio-economic and sex differences in selected health-related fitness measures in 9-12-year-old children. In this study, there was a large variance in WIMD scores ( $\mathrm{SD}=598.6$ ) to classify socio-economic quintiles, with children living in one of the most deprived (WIMD $=23$ ), to the most affluent areas $($ WIMD $=1908)$ in Wales.

The current results showed that, regardless of sex, children in the middle quintile 
(WIMD quintile 3) had a significantly higher mean BMI z-score than their least deprived counterparts (WIMD quintile 5), in contrast to studies that have found no association between school deprivation (school postcode) or individual deprivation levels (free school meals) and weight status in children (Brophy et al., 2012; Dummer et al., 2005). Nonetheless, the current results are in line with a recent study on school children in England, as well as a systematic review, that show an inverse association between socio-economic status and BMI/weight status (Nevill et al., 2017; Shrewsbury \& Wardle, 2008). It is important to note, however, that there were no other significant mean differences in BMI z-score between WIMD quintile groups. In fact, there was little mean difference between WIMD quintiles 1, 2 and 3, as well as between WIMD quintiles 4 and 5. This highlights that there appears to be a split in BMI z-scores, with children in the bottom 3 quintiles (middle to high deprivation), having a higher mean BMI z-score than the top 2 quintiles (least deprived). A similar split between high to middle deprivation and middle to low deprivation was also apparent in a study that showed a significant difference between socio-economic quartile 1 and 2 (least to middle affluent) and quartile 3 and 4 (middle to most affluent) (Fairclough, Boddy, Hackett, \& Stratton, 2009). Since national surveillance evidence shows little difference in physical activity levels across socio-economic status in Wales (Tyler et al., 2016; Welsh Government, 2015a, 2017), it can be speculated that the socioeconomic divide seen in the present study may be related to other factors. One such factor may be dietary intake, as a systematic review provided evidence that low socioeconomic status was associated with poorer diets (Hanson \& Chen, 2007). Further research investigating the concurrent effect of dietary consumption and possible other influencing factors on weight status and fitness by socio-economic status is warranted.

Congruent with previous studies conducted measuring muscular strength and cardiorespiratory fitness in children (Cohen et al., 2010; De Miguel-Etayo et al., 2014; Noonan et al., 2017), the current results showed that boys had a significantly higher grip strength and achieved a significantly greater 20m MSRT score than girls. Moreover, research has shown that boys have higher physical activity levels compared to girls (Tyler et al., 2016; Welsh Government, 2015a, 2017). Given that physical activity has a reciprocal relationship with physical fitness levels (Janssen \& Leblanc, 2010; Ortega et al., 2008), these reported differences may be a contributing factor to the differences in fitness levels between girls and boys seen in the current study.

While muscular strength has been shown to be positively associated with socio-economic status in children (Freitas et al., 2007; Jiménez-Pavón et al., 2010), our results 
were converse in that there was only a significant difference in grip strength between least deprived (WIMD quintile 5) and most deprived (WIMD quintile 1). Moreover, both girls and boys had a significantly higher 20m MSRT score in the least deprived group (WIMD quintile 5) compared to most deprived group (WIMD quintile 1), which agrees with other work (Mutunga et al., 2006). It is noteworthy that significant differences in 20m MSRT score were more apparent with increases in socio-economic status in boys. Specifically, most deprived boys (WIMD quintile 1) had a significantly lower 20m MSRT score than all other groups of boys except for boys in the middle quintile (WIMD quintile 3), while boys in the middle quintile (WIMD quintile 3) had a significantly lower 20m MSRT score than their less deprived counterparts (WIMD quintile 4 and 5). While there is little difference in physical activity levels across socio-economic status in Wales, a difference in sport participation has been reported, with around a ten percent difference in the number of children who are hooked on sport (take part in sport on 3 or more occasions a week in an extracurricular (schoolbased) or a community club setting) in the least deprived compared to most deprived areas in Wales (Sport Wales, 2015c; Tyler et al., 2016). Given that a recent study has shown that, regardless of socio-economic status, participation in sport is associated with better physical fitness when compared to non-participation (Golle, Granacher, Hoffmann, Wick, \& Muehlbauer, 2014), the differences in fitness levels found between the least and most deprived children may be, at least in part, due to differences in sport participation.

Although not the primary aim, the present study enabled comparisons of healthrelated fitness data obtained to international normative values. In comparison to European sex- and age-specific normative values on over 200,000 grip strength test performances of children and young people aged 9-17 years across 30 countries (Tomkinson, Carver, et al., 2017), girls' and boys' mean grip strength score in the present study were in the $50^{\text {th }}$ and $40^{\text {th }}$ centile, respectively. Further, in comparison to international sex- and age-specific normative values on over 1 million 20m MSRT performances of 9-17 year olds from 50 countries (Tomkinson, Lang, Tremblay, et al., 2017), girls and boys achieved a level commensurate with the $40^{\text {th }}$ and $50^{\text {th }}$ centile, respectively. Therefore, cardiorespiratory fitness and muscular strength levels of girls and boys in the current study were low to moderate in comparison to international norms.

The current study showed that inequalities exist in body mass index, muscular strength, and cardiorespiratory fitness across socio-economic status and sex in children. Moreover, overall health-related fitness levels of children involved in the study were low to 
moderate in comparison to international normative values (Tomkinson, Carver, et al., 2017; Tomkinson, Lang, Tremblay, et al., 2017). Given the negative implications of low physical fitness levels on health, focused services and interventions should work to improve physical fitness levels, and specifically aim to close the gap in inequalities, particularly in areas of high deprivation and in girls. These results also provide evidence for the importance spending time in vigorous intensity activities, specifically those that strengthen muscle in children, and indicate that this should be a goal in current and future health promotion policies.

\subsubsection{Limitations}

It is important to note that the current study is not without limitations. The crosssectional study design of this study does not allow for causality to be determined, and therefore future investigations into the causes of (i) the potential socio-economic divide in BMI z-scores, (ii) the more pronounced differences in 20m MSRT by socioeconomic status in boys compared to girls and (iii) the lack of significant difference in 20m MSRT between boys in most deprived and middle quintiles, perhaps considering any differences in physical activity, sport participation and exercise levels across specific groups. Moreover, although the measures used in this study are valid and used in many large-scale studies, future research should aim to include more objective measures of physical fitness (e.g. using treadmill based $\mathrm{VO}_{2}$ peak assessments) and body composition (e.g. skinfolds and circumferences, or dual-energy X-ray absorptiometry (DEXA)), to assess fat and fat-free mass and percentage body fat. However, capturing this information on such large-scale data would be cost and time intensive.

\subsection{Conclusion}

The present study was one of the first large-scale studies to investigate differences in body mass index, muscular strength and cardiorespiratory fitness by sex and different levels of socio-economic status. In accord with previous research, boys had significantly higher muscular strength and cardiorespiratory fitness than girls. Children in the middle quintile (WIMD quintile 3) had a significantly higher mean BMI z-score than those in the least deprived quintile (WIMD quintile 5). Most deprived children (WIMD quintile 1) had significantly lower muscular strength and cardiorespiratory fitness than their least deprived counterparts (WIMD quintile 5). Overall, these results show that inequalities exist between socio-economic status and sex in selected health-related fitness measures and should be used to inform focused services and interventions to improve current health inequalities and future health implications. 


\subsection{Summary: Study 3}

This study aimed to investigate socio-economic and sex differences in selected healthrelated fitness measures in 9-12-year-old children.

Data were captured on 3,407 children (49.3\% boys; aged $10.5 \pm 0.6$ years) as part of the serial Swan-Linx programme between 2013-2017. Three components of healthrelated fitness were measured, namely cardiorespiratory fitness $(20 \mathrm{~m}$ multistage shuttle run test; 20m MSRT), muscular strength (handgrip strength) and body mass index (stature and body mass). Participants' home postcodes were used to calculate Welsh Index of Multiple Deprivation (WIMD) scores, and to split participants into quintile groups. A two-way Analysis of Variance examined differences in BMI z-score by sex and WIMD quintiles. Two-way Analysis of Covariances (ANCOVAs) investigated the effect of sex and WIMD quintiles on grip strength, adjusting for BMI z-score, and on shuttles achieved in the 20m MSRT, adjusting for maturation.

Regardless of sex, children in the middle quintile had a significantly higher mean BMI $\mathrm{z}$-score $(\mathrm{p}=0.029)$ than their least deprived counterparts. There was a significant increase in grip strength $(\mathrm{p}=0.005)$ and 20m MSRT (boys $\mathrm{p}<0.001$; girls $\mathrm{p}=0.028$ ) between most and least deprived quintiles. Significant differences in 20m MSRT score were more apparent with increases in socio-economic status in boys. Overall, results showed that inequalities exist and should be used to inform focused services and interventions to improve current and future health inequalities. 


\section{Thesis Map}

Study

\section{Outcomes}

\begin{tabular}{|c|c|c|c|}
\hline 1 & $\begin{array}{l}\text { Results from the Active Healthy } \\
\text { Kids Wales } 2016 \text { Report Card on } \\
\text { Physical Activity for Children and } \\
\text { Youth }\end{array}$ & Key Findings & $\begin{array}{l}\text { To consolidate and translate research related } \\
\text { to physical activity among children and } \\
\text { youth in Wales and to synthesise national } \\
\text { surveillance data from several surveys in } \\
\text { order to assign grades to physical } \\
\text { activity/inactivity behaviours and influences } \\
\text { indicators, as well as to examine trends or } \\
\text { inequalities in the data. } \\
\text { Grades were assigned as follows; overall } \\
\text { physical activity levels, D+; organized sport } \\
\text { participation, C; active and outdoor play, C; } \\
\text { active transportation, C; sedentary } \\
\text { behaviours, D-; physical literacy, INC; } \\
\text { family and peer influences, D+; school, B; } \\
\text { community and the built environment, C; } \\
\text { and national government policy, strategies } \\
\text { and investments, B-. }\end{array}$ \\
\hline 2 & $\begin{array}{l}\text { Ten-year Secular Changes in } \\
\text { Selected Health and Fitness } \\
\text { Parameters of 10-11 Years Old } \\
\text { Swansea School Children - 2003- } \\
2013\end{array}$ & Key Findings & $\begin{array}{l}\text { To highlight the secular changes in selected } \\
\text { health and fitness measures (body mass } \\
\text { index, prevalence of overweight and obesity, } \\
\text { and grip strength) in } 10-11 \text { year old Swansea } \\
\text { school children, using data obtained in } 2003 \\
\text { and } 2013 \text {. } \\
\text { BMI z-scores, as well as prevalence of } \\
\text { overweight and obesity, decreased } \\
\text { significantly between } 2003 \text { and } 2013 \text { for } \\
\text { both boys and girls. After BMI z-scores } \\
\text { adjustment, grip strength decreased } \\
\text { significantly for boys, but not for girls. }\end{array}$ \\
\hline \multirow[t]{2}{*}{3} & $\begin{array}{l}\text { A Cross-Sectional Study on the } \\
\text { Socio-economic and Sex } \\
\text { Differences in Health-Related } \\
\text { Fitness Measures in School } \\
\text { Children }\end{array}$ & Aim & $\begin{array}{l}\text { To investigate socio-economic and sex } \\
\text { differences in selected health-related fitness } \\
\text { measures (body mass index, MSRT, grip } \\
\text { strength) in 9-12-year-old children. }\end{array}$ \\
\hline & & $\begin{array}{c}\text { Key } \\
\text { Findings }\end{array}$ & $\begin{array}{l}\text { Regardless of sex, children in } \\
\text { the middle quintile had a } \\
\text { significantly higher mean BMI } \\
\text { z-score than their least deprived } \\
\text { counterparts. There was a } \\
\text { significant increase in grip } \\
\text { strength and } 20 \mathrm{~m} \text { MSRT } \\
\text { between most and least deprived } \\
\text { quintiles. Significant differences } \\
\text { in } 20 \mathrm{~m} \text { MSRT score were more } \\
\text { apparent with increases in } \\
\text { socio-economic status in boys. }\end{array}$ \\
\hline
\end{tabular}




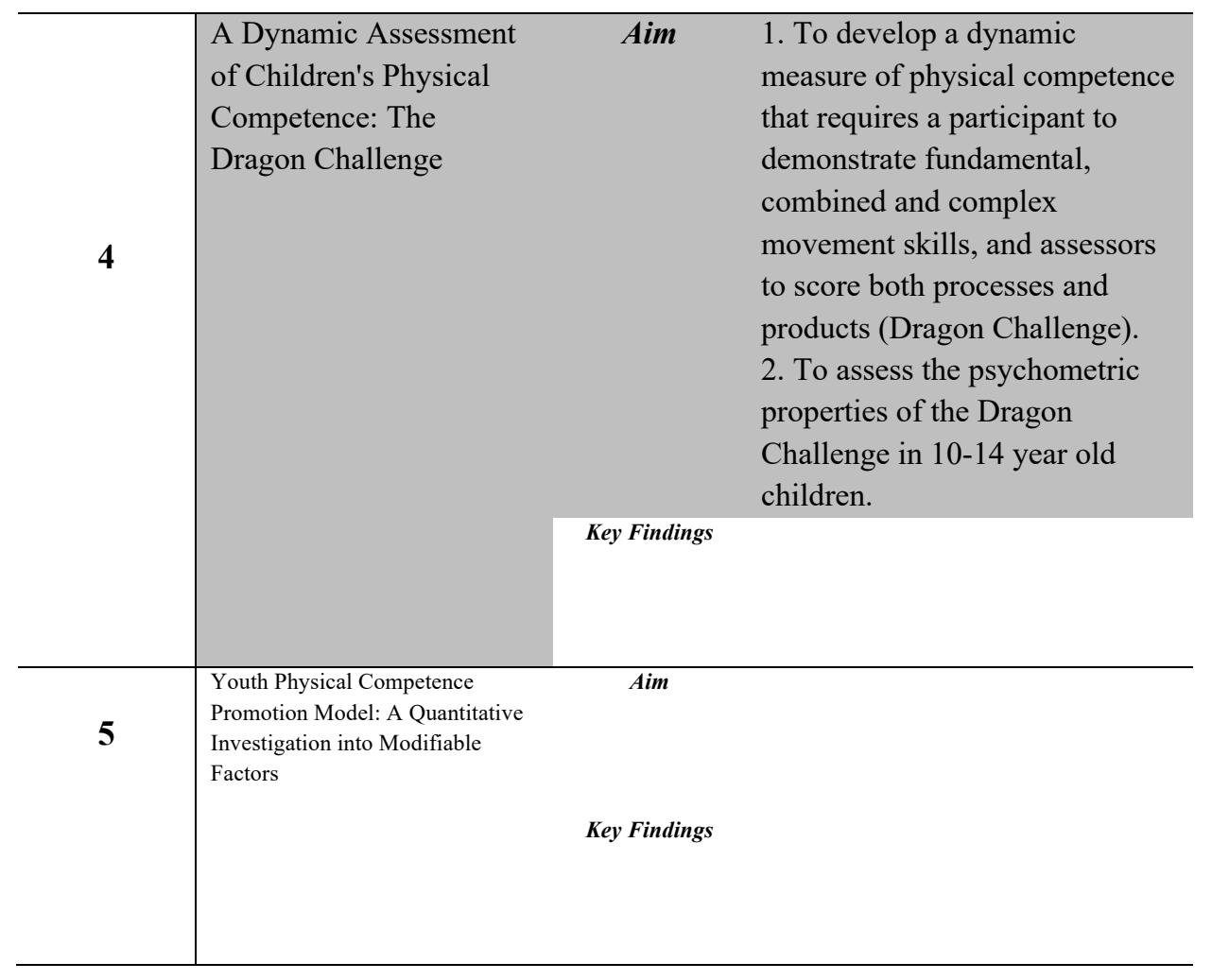




\section{Study 4}

In the previous years, two task and finish groups (in 2001 and 2013) recommended to Welsh Government that 'physical literacy' should be the highest priority in education, sport and health policy. Consequently, Sport Wales acted as the lead organisation in promoting physical literacy within Wales. The organisation received significant funding from the Welsh Government to deliver long term improvements in physical literacy and to increase physical activity among children in schools in Wales, helping younger generations to be hooked on sport for life and to adopt healthy lifestyles.

In 2013, Sport Wales convened a Physical Literacy Expert group comprising of academics and professionals from schools, community, sport, and health, who created a physical literacy framework to drive the work across Wales forward. Two main outcomes from this group were; 1) that physical literacy was a lifelong concept and needed to be considered using a 'cradle to grave' approach, and 2) that a stronger evidence base was required on physical literacy, particularly on school children. As such, there was a growing interest from parents, teachers, sports coaches, health professionals and researchers regarding children's levels of physical literacy and in particular physical competence. In the Sport Wales School Sport Survey, additional questions related to physical literacy such as confidence in trying new activities (confidence) and whether PE and school sport helped them to follow a healthy lifestyle (knowledge and understanding), were included and completed by children and young people. While these questions were informative on assessing parts of the physical literacy spectrum (confidence, knowledge and understanding), there was no measure of physical competence. As a result, academics from Swansea, Liverpool John Moores and Glyndwr Universities worked with practitioners and professionals from schools and community sport to develop a robust measure of physical competency for 10-14 year old children - the Dragon Challenge. Study 4 discusses the development, validity and reliability of this tool.

\subsection{A dynamic assessment of children's physical competence: The Dragon Challenge}

* This chapter is a published manuscript:

Tyler, R., Foweather, L., Mackintosh, K. A., \& Stratton, G. (2018). A Dynamic Assessment of Children's Physical Competence. Medicine \& Science in Sports \& Exercise, 50(12), 2474-2487. https://doi.org/10.1249/MSS.0000000000001739 


\subsection{Introduction}

The International Physical Literacy Association defines physical literacy as the motivation, confidence, physical competence, knowledge and understanding to value and take responsibility for engagement in physical activities for life (International Physical Literacy Association, 2016). Such a definition describes the multidimensional and complex nature of physical literacy, highlighting the purported importance of physical literacy as a precursor to physical activity (Edwards et al., 2016). Therefore, given that physical activity has been shown to result in numerous health benefits (Jurbala, 2015), the promotion of physical literacy is fundamental for physical activity-associated health benefits. According to Lundvall (2015), accurate assessment of physical literacy is essential, and there is a need to develop valid tools that effectively and efficiently assess each of the affective, cognitive, and psychomotor domains in order to evaluate whether programmes are successful (Corbin, 2016).

One of the key elements of physical literacy is physical competence, which, even within itself, is a multidimensional concept. Whitehead (p204; 2010), describes physical competence as "the sufficiency in movement vocabulary, movement capacities and developed movement patterns plus the deployment of these in a range of movement forms." Specifically, movement vocabulary refers to the repertoire of movements that one can perform, and can be expanded through experience and progressive challenge in the deployment of a wide range of movement capacities/skills and movement patterns (Whitehead, 2010).

Movement capacities are the integral abilities that make it possible to improve and develop physical competence (Whitehead, 2010). These capacities or skills consist of three interrelated constructs: fundamental or simple movement skills (FMS) (balance, core stability, coordination, speed variation, flexibility, control, proprioception, and power), combined movement (poise, fluency, precision, dexterity, and equilibrium), and complex movement (bilateral coordination, inter-limb coordination, handeye coordination, turning, twisting and rhythmic movements, and control of acceleration/deceleration) (Giblin et al., 2014; Whitehead, 2010). FMS comprise locomotor skills (moving the body in any direction from one point to another), stability skills (balancing the body in one place or while in motion), and object control/manipulative skills (handling or controlling objects with the hand, foot, or an implement) (Gallahue et al., 2012; Giblin et al., 2014; Whitehead, 2010). Children have the potential to master FMS by 7-8 years of age, with FMS developing rapidly between 3 and 8 years (Gallahue et al., 2012). 
The procurement of movement capacities/skills and the ability to utilise them to produce movement patterns are essential for the development of physical competence within physical literacy capability (Whitehead, 2010). Movement patterns, described as general (e.g., sending, striking, receiving, running, jumping, rotating), refined (e.g., throwing, dribbling, catching, sprinting, hopping, turning) and specific (i.e. sportspecific movement patterns), are amalgamations of movement that stem from the selection and application of movement skills (Whitehead, 2010). More refined and specific movement patterns are achieved when fundamental, combined and complex movement skills are utilised (Corbin, 2016; Giblin et al., 2014; Whitehead, 2010). There is therefore much need to develop combined and complex movement skills, to take part in more advanced physical activities in a variety of settings (i.e., land, water, air, ice) (Jurbala, 2015; Whitehead, 2010) and movement forms (i.e., adventure, aesthetic, athletic, competitive, fitness and health, interactional/relational) (Whitehead, 2010), and thus this development is posited to be a foundation stone in developing physical literacy in maturing children (Corbin, 2016; Giblin et al., 2014).

Whilst many existing land-based movement skill assessments measure physical competence (Giblin et al., 2014; Robinson \& Randall, 2017), the majority involve the performance of discrete skills in isolation (e.g., the Test of Gross Motor Development (TGMD-2/3) (Ulrich, 2000), the Bruininks-Oseretsky Test of Motor Proficiency, Second Edition (BOT-2) (Bruininks \& Bruininks, 2005), the Movement Assessment Battery for Children-2 (MABC-2) (Henderson et al., 2007), CS4L: Physical Literacy Assessment for Youth Fun (PLAYfun) (Cairney et al., 2018), Passport for Life: Movement Skills Assessment (Physical and Health Education Canada (PHE Canada), 2014)). This static testing environment limits transferability and applicability to multiskill and sport environments and does not assess combined and complex movement skills (Giblin et al., 2014). Moreover, it has been suggested that considering skills in isolation ignores a constraints-based approach (Newell, 1986), in which environmental constraints are taken into account, and by doing so this approach is not 'authentic'. An authentic environment is one that is developmentally-appropriate and considers the interaction of the individual and the environment, as well as the specified movement skill (Barnett, Stodden, et al., 2016; Newell, 1986). Performance of movement skills in isolation does not incorporate the measurement of an individuals' ability to alter and combine movement skills according to the task at hand and the environment, both of which are important traits to advance physical competence and progress one's physical literacy (Whitehead, 2010). Finally, assessments that measure skills in iso- 
lation have also been criticised for being time- and resource-intense (Giblin et al., 2014; Wiart \& Darrah, 2001). Thus, tools that measure physical competence in children aged over 8 years should assess fundamental, combined and complex movement skills in a dynamic and more authentic environment, in an efficient manner. The assessment of refined and specific movement patterns in a variety of novel combinations and complexities will more accurately reflect physical competence.

Physical competence can be evaluated by process- or product-based assessments (Bruininks \& Bruininks, 2005; Cairney et al., 2018; Henderson et al., 2007; Physical and Health Education Canada (PHE Canada), 2014; Ulrich, 2000). Primarily processbased assessments (e.g., TGMD-2, CS4L: PLAYfun, Passport for Life: Movement Skills Assessment) measure how children move and provide qualitative information on the technique of the movement patterns (Hardy et al., 2010). This type of assessment can be sensitive to assessor experience and subjectivity (Schoemaker et al., 2012). On the other hand, assessments that are primarily product-based (e.g., MABC2, BOT-2) are usually quantitative and focus on the outcome of the movement (Logan et al., 2017), but potentially lack the sensitivity needed to identify individual differences in movement abilities (Giblin et al., 2014). The equivocal relationship between process- and product-based assessments of physical competence has resulted in the use of combined assessments for measuring physical competence (Logan et al., 2017; Robinson et al., 2015; Rudd et al., 2016). Therefore, a single assessment that aims to equally assess both the process/technique and the product/outcome aspects of physical competence is warranted.

The assessment of physical competence can be formative or summative. Specifically, formative assessments measure current levels of performance to identify a baseline and the individual needs of children, enabling the development of an educational programme catered to those children, whereas summative assessments are used to measure progress of a child at the end of a period of education (Gallahue \& Donnelly, 2007). Therefore, a physical competence assessment tool developed within the context of education, should aim to be both formative and summative, so that it can be used as a self-referenced assessment, which is able to compare a child's pre- and posteducational programme performance.

Recently, the Canadian Agility Movement Skill Assessment (CAMSA) was developed and validated to assess physical competence in 8-12-year-old children for surveillance, as well as examining movement skills over time (Longmuir et al., 2017). This 
assessment requires a series of seven movement tasks (two-footed jump, side slide, catch, throw, skip, hop, and kick) to be completed in a continuous dynamic obstacle course to create a more authentic environment and to assess combined and complex movement skills. Performances are assessed using the time taken to complete an obstacle course consisting of 14 process/technique- and product/outcome-based criteria (Longmuir et al., 2017). Whilst this assessment has shaped the way towards assessing movement skills in a dynamic fashion, there are noteworthy design limitations of the CAMSA. For example, the course does not include any specific stability movement skill tasks and there are a greater number of locomotor movement skill tasks than object control movement skill tasks. In addition, the scoring is unbalanced between locomotor and object control criterion, as well as between product- and process-based criterion. As such, an assessment targeting older aged children and adolescents (1014 years), with a more balanced design, is warranted.

Therefore, the first aim of this study was to develop a dynamic assessment to measure elements of physical competence (Dragon Challenge; DC), that requires the demonstration of fundamental (e.g., balance), combined (e.g., poise) and complex (e.g., rhythmic movements) movement skills through refined (complex) and specific movement patterns (e.g., hopping, turning, jumping patterns), measured by both product/ outcome- and process/technique-based evaluations. The study sought to produce an assessment that would be feasible for national surveillance and could be used as both a formative and summative assessment in the educational context. The second aim of the study was to assess the psychometric properties of the DC in measuring physical competence in children, including construct and concurrent validity and test-re-test and inter- and intra-rater reliability, as per American Educational Research Association, American Psychological Association, and National Council on Measurement in Education guidelines.

\subsection{Methods}

This study involved three phases. Phase one included the development of the DC, including the review process to establish face and content validity. Phase two included gathering surveillance data and establishing construct validity and phase three involved investigating concurrent validity, test-retest, inter-rater and intra-rater reliability. The COSMIN (Consensus-based Standards for the Selection of Health Measurement Instruments) framework was used to guide the design and evaluate the methodological quality (Mokkink et al., 2010). This study would achieve a quality level of good to excellent on the COSMIN rating system. The protocol, validation and relia- 
bility study of the DC were approved by the institutional Research Ethics Committee (PG/2014/37 \& PG/2014/39). Informed parental consent and participant assent were obtained prior to participation.

\subsubsection{Phase 1. Development of the DC}

Programme of Research to Develop the DC: Paediatric exercise science academics, practitioners, and professionals from schools and community sport $(n>30)$ co-designed a land-based measure of elements of physical competence in children (10-14 years of age) that was aligned to physical education and sport coaching school and community programmes that aimed to promote physical literacy. The circuit of tasks were collectively named the 'Dragon Challenge' to align with the Sport Wales' Dragon multiskills and sport initiative (http://sport.wales/community-sport/education/ dragon-multiskills-sport.aspx). The DC assessment tool underwent several stages of development. The first stage involved desk research, where an initial review was conducted on existing movement skill assessment tools that inform physical competence (Bruininks \& Bruininks, 2005; Gallahue et al., 2012; Henderson et al., 2007; NSW Department of Education and Training, 2000; Ulrich, 2000). From this, each of the 10 tasks/skills in the first protocol of the DC were examined for initial content validity. Subsequently, the second stage involved an iterative process of designing and testing the DC, whereby each task and its subsequent process- and product-based criteria were defined, with significant input from expert practitioners in physical education and community sport from across Wales $(n>30)$. This stage included six iterations of protocol development, with the overall aim being to refine and assess the suitability of tasks, and to establish whether each individual task, and the overall assessment tool, could be used as an appropriate measure of children's physical competence. The initial tasks selected were therefore modified to incorporate refined and specific movement patterns that would adequately challenge children's fundamental, combined and complex movement skills, developed during physical education curriculum and the Dragon Sport multi-skill and sport initiative. The protocol development process was completed over a 12-month period (July 2013 to 2014). Two hundred and eighty-eight children aged 10-12 years took part in the DC pilot testing days. The final DC protocol included nine tasks ordered to create continuity of movement and allow assessors to accurately observe children's performances (see Appendix VI). Process/technique and product/outcome indicators for the assessment criteria were continuously developed and refined by discussion and consensus until the DC was finalised.

Establishing Face and Content validity: Face and content validity refers to how well a 
specific assessment measures what it intends to measure. The group of University paediatric exercise specialists, with expertise in physical education, physical competence and physical literacy research were involved in reviewing the DC. Face and content validity was qualitatively reviewed by a trained researcher (Lawrence Foweather) with over 10 years' experience of physical competence and movement skill assessment. In addition, internationally recognised experts $(n=5)$ in childhood movement skill, fitness, and physical literacy assessment within the personal networks of this researcher, advised Lawrence Foweather and provided comments (in confidence) to inform the review process.

The review process comprised of in situ observations of children's performances, and a subjective analysis of the assessment protocol. Checks were made for the inclusion of critical movement tasks in accordance with a developmentally-appropriate assessment of physical competence through comparisons with existing assessment tools (Bruininks \& Bruininks, 2005; Gallahue et al., 2012; Henderson et al., 2007; NSW Department of Education and Training, 2000; Ulrich, 2000). Further checks were made to ensure that the DC circuit of tasks were in line with physical education curriculum content for children in this age range (10-14 years old), in that it required the utilisation of fundamental, combined, and complex movement capacities/skills to perform refined and specific movement patterns. Finally, clarity in behavioural definition (descriptions of the movement characteristics associated with the performance of each task) used in the assessment criteria was ensured.

Face validity: Children complete the nine DC tasks in a set sequence; Table 9 shows the primary and secondary skill types necessary for each component. Several tasks (five out of nine) require children to perform a combination of skills and movement patterns, to demonstrate competence. Components of motor fitness such as agility, balance, coordination, strength, power, speed and reaction time are all widely utilised within the DC. The DC challenges children to demonstrate movement skills and motor fitness in combinations of different movement patterns and in continuous fashion as opposed to discrete skills in assessments such as the TGMD-2 or MABC-2. Further, children are required to demonstrate movement concepts and attributes expected of a physically competent person, (i.e., "movement with poise, economy and confidence in a wide variety of challenging situations" and "sensitive perception in 'reading' all aspects of the physical environment, anticipating movement needs or possibilities and responding appropriately to these, with intelligence and imagination") (Whitehead, 2010). Thus, the DC tasks were representative of multiple elements of physical com- 
petence.

Table 9. Description of Dragon Challenge protocol and tasks and types of skills utilised during each task

\begin{tabular}{|c|c|c|c|c|}
\hline DC Task & Description & Stability & Locomotor & Object Control \\
\hline $\begin{array}{l}\text { 1. Balance } \\
\text { Bench }\end{array}$ & $\begin{array}{l}\text { Runs to bench. Walks length of narrow side of bench } \\
\text { beam, completing a } 360^{\circ} \text { turn at mark before } \\
\text { dismounting at the end of the bench and returning to } \\
\text { iPad. If the child falls off then task is ended and the } \\
\text { child returns to iPad immediately. }\end{array}$ & - & 0 & \\
\hline $\begin{array}{ll}\text { 2. } & \text { Core } \\
& \text { Agility }\end{array}$ & $\begin{array}{l}\text { Runs to gym mat. Completes } 4 \text { positions (dish on back - } \\
\text { arch on front - dish on back - arch on front), rotating } \\
\text { both ways. Returns to iPad. }\end{array}$ & $\bullet$ & & \\
\hline $\begin{array}{ll}\text { 3. Wobble } \\
\text { Spot }\end{array}$ & $\begin{array}{l}\text { Runs to wobble spot and picks up bean bag on floor. } \\
\text { Completes } 5 \text { bean bag passes around body whilst } \\
\text { balancing on wobble spot on one leg. Returns to iPad. If } \\
\text { child falls off after starting, the task is ended and the } \\
\text { child returns to the iPad immediately. }\end{array}$ & • & & 0 \\
\hline $\begin{array}{l}\text { 4. Overarm } \\
\text { Throw }\end{array}$ & $\begin{array}{l}\text { Collects tennis ball from hoop. Overarm throw at target } \\
\text { from badminton court service box line approx. 10m } \\
\text { from target. The child does not collect ball and returns } \\
\text { to iPad. }\end{array}$ & & & $\bullet$ \\
\hline $\begin{array}{l}\text { 5. Basketball } \\
\text { Dribble }\end{array}$ & $\begin{array}{l}\text { Collects basketball from hoop. Dribbles around } \\
\text { coloured spots on floor in } z \text { formation (body and ball } \\
\text { move around outside of spots) with either hand. After } \\
\text { dribbling around last spot, finishes with a dribble down } \\
\text { the middle, returning ball to hoop/iPad. }\end{array}$ & & 0 & $\bullet$ \\
\hline 6. Catch & $\begin{array}{l}\text { Runs forward and collects tennis ball from floor. } \\
\text { Underarm throws ball against rebound net to catch from } \\
\text { any distance without a bounce. Does not collect ball if } \\
\text { dropped. Returns to iPad. }\end{array}$ & & 0 & $\bullet$ \\
\hline 7. T-Agility & $\begin{array}{l}\text { Completes t-agility run, facing forwards throughout. } \\
\text { Returns to iPad. }\end{array}$ & 0 & $\bullet$ & \\
\hline $\begin{array}{ll}\text { 8. Jumping } \\
\text { Patterns }\end{array}$ & $\begin{array}{l}\text { Runs to coloured foot markers and hurdles. Follows } \\
\text { jumping pattern sequence to finish (2-footed jump over } \\
\text { hurdle } \rightarrow 2 \text {-footed landing } \rightarrow 2 \text { left hops } \rightarrow 2 \text { right } \\
\text { hops } \rightarrow 2 \text {-footed jump over hurdle } \rightarrow 2 \text {-footed landing. } \\
\text { Returns to iPad. }\end{array}$ & 0 & • & \\
\hline 9. Sprint & $\begin{array}{l}\text { Runs through start gate and then } 10 \mathrm{~m} \text { sprint } \\
\text { acceleration to finish line. }\end{array}$ & & • & \\
\hline
\end{tabular}

Content Validity: Internationally recognised experts $(n=5)$ in childhood movement skill, fitness, and physical literacy assessment, confirmed that the DC was a valid and practical measure of physical competence, and that each task was challenging, achievable, and age-appropriate. Further, the tool was praised for its feasibility and efficiency. 
DC task design: Balancing, running, hopping, jumping, throwing, dribbling, catching and sprinting are common skills that are assessed in isolation within existing movement skill assessment tools (Bruininks \& Bruininks, 2005; Gallahue et al., 2012; Henderson et al., 2007; NSW Department of Education and Training, 2000; Ulrich, 2000). Whilst the DC incorporates these skills and others, it is conducted in a continuous fashion within a timed trial, thus tasks are dynamic, sequential and include additional layers of complexity. The order of the tasks is standardised (as displayed in Table 9) but children perform the challenge under the illusion that the order is random, except for the final task, which is always the sprint (note, the full demonstration is in a different order to the standardised protocol). Each subsequent task is displayed on an iPad/tablet. Thus, the DC also explores perception-action coupling, as participants must coordinate recognising environmental information and the associated movement responses to such information, in order to complete the goal of each task.

Children observed a demonstration of each DC task and then the full DC. An introduction and demonstration video (see Appendix VI) of the DC was produced to ensure consistent administration and adequate demonstrations of the tasks were provided to the children in line with those outlined in the DC manual. In addition, the full video of the completion of the DC (see Appendix VI) could be shown. Children were given two practice attempts at each challenge task but they did not practise the challenge in full.

Children typically took between 90 and 240 seconds to complete the DC. An assessor used a stopwatch to record completion time (to nearest $0.1 \mathrm{~s}$ ). Each assessment required at least one trained assessor and one administrator. An additional assistant was required to supervise the non-participating children. The space requirement was designed to fit within the dimensions of a full-sized badminton court (13.4m x $6.1 \mathrm{~m})$, which most school gymnasiums and community sports centres are likely to have. Taken together, including set-up (15 minutes), the viewing of the videos and questions (26 minutes for a full group), and practice and completion of DC (approximately 10 to 12 children in 60 minutes), the total assessment time per child was approximately 10 minutes. For further information on the DC assessment including equipment list and descriptions of the assessment, see Appendix V.

DC assessment criteria: The DC indicators included both technical (process) and outcome (product) characteristics of movement performance (Table 10). Due to the 
challenges of real-time observation, the number of criterion to be assessed was limited to three per task (i.e., two technical/process criteria and one outcome/product criteria). Given that there were several technical characteristics that could be examined for each task, it was important that assessment criteria represented critical features of movement. Existing assessment tools and reference to developmental sequences were used to inform these decisions (Bruininks \& Bruininks, 2005; Gallahue et al., 2012; Henderson et al., 2007; NSW Department of Education and Training, 2000; Ulrich, 2000). A global review of the criteria (Table 10) suggested that the majority assess important characteristics of each task.

The DC was scored in three ways in accordance with the instructions specified within the DC manual (see Appendix V): (1) TECHNIQUE - 1 point was given for each of the technical/process criterion $(n=18)$ successfully demonstrated by the child (2) OUTCOME - 2 points were awarded for each outcome/product criterion $(n=9)$ successfully demonstrated by the child, and (3) TIME - time taken to complete the DC was recorded and converted to a score (higher scores for faster time). Each of these constructs (technique, outcome, and time) was scored out of 18 in order to be equally weighted, and then summed to give a total score (DC total score $=54$ ). Cut-points were also produced for the DC total score using the $33^{\text {rd }}, 66^{\text {th }}$ and $95^{\text {th }}$ percentiles based on pilot data collected across Wales in 2015. These percentile thresholds were selected to categorise typically-developing 10-12 year old children into Bronze, Silver, Gold and Platinum bands, thus making results easier to interpret by children, coaches, teachers, and parents. 
Table 10. Dragon Challenge assessment criteria

\begin{tabular}{|c|c|c|c|}
\hline DC Task & Technique Criterion & Technique Criterion & Outcome Criterion \\
\hline 1. Balance Bench & $\begin{array}{l}\text { 1.1 Moves without hesitation } \\
\text { up to turn }\end{array}$ & $\begin{array}{l}\text { 1.2 Body posture stable } \\
\text { (head \& trunk stable, minimal } \\
\text { arm flailing) }\end{array}$ & $\begin{array}{l}\text { 1.3 Walks length of beam, } \\
\text { completes full turn at } 3 / 4 \text { mark } \\
\text { without falling off, dismounts at } \\
\text { end zone }\end{array}$ \\
\hline 2. Core Agility & $\begin{array}{l}2.1 \text { Hands \& legs extended \& } \\
\text { held with tension, with } \\
\text { shoulders \& feet off the floor }\end{array}$ & $\begin{array}{l}\text { 2.2 Controlled \& fluent } \\
\text { transition through shapes }\end{array}$ & $\begin{array}{l}2.3 \text { Completes } 4 \text { positions in } \\
\text { correct order } \\
\text { (dish on back - arch on front - } \\
\text { dish on back - arch on front), } \\
\text { rotating both ways }\end{array}$ \\
\hline 3. Wobble Spot & $\begin{array}{l}\text { 3.1 Non-support foot does not } \\
\text { touch support leg/foot/wobble } \\
\text { spot/floor }\end{array}$ & $\begin{array}{l}\text { 3.2 Body \& head are } \\
\text { stable/still }\end{array}$ & $\begin{array}{l}\text { 3.3 Completes } 5 \text { bean bag passes } \\
\text { around body whilst balancing on } \\
\text { wobble spot on one leg } \\
\text { \# 'correct' passes } \begin{array}{lllllll}0 & 1 & 2 & 3 & 4 \\
5\end{array}\end{array}$ \\
\hline 4. Overarm Throw & $\begin{array}{l}\text { 4.1 Throwing arm moves in a } \\
\text { backward arc to initiate throw } \\
\text { (shoulder rotates) }\end{array}$ & $\begin{array}{l}\text { 4.2 Steps with the foot } \\
\text { opposite throwing hand } \\
\text { towards target }\end{array}$ & $\begin{array}{l}4.3 \text { Overarm throw directly hits } \\
\text { target (ball should not bounce } \\
\text { prior to hitting target) }\end{array}$ \\
\hline 5. Basketball Dribble & $\begin{array}{l}\text { 5.1 Pushes ball with fingertips } \\
\text { (not slapping at the ball) }\end{array}$ & $\begin{array}{l}\text { 5.2 Controlled directional } \\
\text { dribbling }\end{array}$ & $\begin{array}{l}\text { 5.3 Dribbles around all spots } \\
\text { using either hand. (body \& ball } \\
\text { must move around outside of } \\
\text { spots). Cannot catch ball/use two } \\
\text { hands simultaneously }\end{array}$ \\
\hline 6. Catch & $\begin{array}{l}6.1 \text { Feet move in line with } \\
\text { rebound }\end{array}$ & $\begin{array}{l}6.2 \text { Catches ball with hands } \\
\text { only (must be caught without a } \\
\text { bounce) }\end{array}$ & $\begin{array}{l}6.3 \text { Successful catch off rebound } \\
\text { net } \\
\text { (must be caught without a } \\
\text { bounce) }\end{array}$ \\
\hline 7. T-Agility & $\begin{array}{l}\text { 7.1 Plants \& drives off outside } \\
\text { foot } \\
\text { (right to left \& left to right) }\end{array}$ & $\begin{array}{l}\text { 7.2 Side-stepping on balls of } \\
\text { feet (right to left \& left to } \\
\text { right; feet don't cross) }\end{array}$ & $\begin{array}{l}7.3 \text { Moves through all points of } \\
\text { 'T' facing forwards (must enter } \\
\text { both right \& left court tramlines) }\end{array}$ \\
\hline 8. Jumping Patterns & $\begin{array}{l}\text { 8.1 Arms drive over first } \\
\text { hurdle (elbows bent \& arms } \\
\text { swing to produce force) }\end{array}$ & $\begin{array}{l}8.2 \text { Rhythmical pattern } \\
\text { throughout }\end{array}$ & $\begin{array}{l}8.3 \text { Completes jumping pattern } \\
\text { sequence correctly. No contact } \\
\text { with hurdles }\end{array}$ \\
\hline 9. Sprint & $\begin{array}{l}\text { 9.1 Drives off balls of feet, } \\
\text { leaning forwards }\end{array}$ & $\begin{array}{l}\text { 9.2 Arms bent, driving } \\
\text { forward \& backwards (arms } \\
\text { bent at approx. right angles) }\end{array}$ & $\begin{array}{l}9.3 \text { Runs through start gate \& } \\
\text { then through to finish (must be } \\
\text { running not walking) }\end{array}$ \\
\hline
\end{tabular}




\subsubsection{Phase 2. Surveillance Data and Construct Validity}

Participants and Procedures: During the development process, a workforce of physical educators, coaches and other professionals in related areas, were trained to implement the DC assessments across four regions of Wales: South East, Mid \& West, Central, and North. At least two assessors from each region received $>20$ hours of training led by Lawrence Foweather, and were only permitted to do assessments once reaching an $85 \%$ level of agreement (3 errors per child) with Lawrence Foweather. This workforce acted as 'gold standard assessors' within their respective region, and rolled out training to their constituents, with use of a gold standard training package for other professionals to be assessed against. In total, circa 200 assessors were trained across the four regions. Trained regional teams then conducted DC assessments in schools between January 2015 and November 2016.

The DC was scored in accordance with the instructions specified within the DC manual. For comparison purposes, technique and outcome scores were also summed to give sub-category scores for tasks primarily utilising stability (sum of technique and outcome criteria in tasks 1-3), object control (sum of technique and outcome criteria in tasks 4-6), and locomotor skills (sum of technique and outcome criteria in tasks 7-9; Table 9). Overall, data were successfully collected for analysis on 4,355 participants from 66 schools, aged 10-12 years from Central South Wales $(n=875)$, South East Wales $(n=1,238)$, Mid and West Wales $(n=1,336)$ and North Wales $(n=906)$. Within this overall sample, $49.9 \%$ of participants were boys, $7.2 \%$ were black and minority ethnic, $20.7 \%$ classified as special educational needs/additional learning needs status and $13.2 \%$ received free school meals (a proxy measure used in Wales for social economic status).

Construct Validity: To ascertain whether the DC behaves according to motor development theory (Gallahue et al., 2012), total, technique/process, outcome/product, and time scores, as well as successful demonstration of each criterion, were examined by sex (boys expected to have higher scores than girls) and age/school level differences (older children expected to achieve higher scores than younger children). The factor structure of the DC was also examined. As each of the nine DC tasks required combinations of movement skills (Table 10), it was hypothesised that the outcome may not produce a 3-factor structure (namely, stability, object control and locomotor), but instead produce a structure with a greater number of factors, each representing a distinct combination of skills. It was also hypothesised that these factors would load on to a higher order factor, namely physical competence. 


\subsubsection{Phase 3. Concurrent Validity and Reliability}

Participants and Procedures: A convenience sample of 50 participants (52\% boys) aged $12.66 \pm 1.51$ years from two schools performed the DC twice with a one-week interval between the two DC data collection days. Participants were from school year $5(\mathrm{n}=8 ; 10.32 \pm 0.31$ years $)$, year $6(\mathrm{n}=8 ; 11.28 \pm 0.32$ years $)$, year $7(\mathrm{n}=10 ; 12.42$ \pm 0.23 years $)$, year $8(n=12 ; 13.48 \pm 0.25$ years $)$, and year $9(n=12 ; 14.51 \pm 0.26$ years) and had a mixture of abilities according to their physical education teacher. Each attempt at the DC was video recorded using two tripod-mounted video cameras [Sony Handycam, Model HDR-PJ410, Sony Corporation, Tokyo, Japan]. Scoring was completed by an expert assessor ( $>50$ hours of DC training and in situ experience), trained assessor (20 hours of DC training and in situ experience), and/or newly trained assessor (5 hours of DC training), in accordance with the instructions specified within the DC manual. For comparison purposes, sub-category scores were also calculated for tasks primarily utilising stability skills, object control skills, and locomotor skills.

On a separate day, participants performed two trials of the Test of Gross Motor Development-2 (TGMD-2) (Ulrich, 2000) and the Stability Skills Assessment (SSA) (Rudd et al., 2015), previously validated movement skills assessments, which required the completion of six locomotor (run, gallop, hop, leap, horizontal jump, and slide) and six object control (striking a stationary ball, stationary dribble, catch, kick, overhand throw, and underhand roll) subtest skills, and three gymnastics training stability skills (rock, log- roll, and back support), respectively. Participants were video recorded using two tripod-mounted video cameras [Sony Handycam, Model HDR-PJ410, Sony Corporation, Tokyo, Japan]. A trained assessor scored the video footage based on the presence $(=1)$ or absence $(=0)$ of three to five component (process) criteria for each of the skills in both trials of the TGMD-2 and SSA (Rudd et al., 2015; Ulrich, 2000). 'Overall skill scores', the cumulative criteria scores for each skill across both trials, were calculated for each of the TGMD-2 and SSA tasks. 'Overall skill scores' for each of the TGMD-2 (=0-96) and SSA (=0-24) tasks were summed to give a 'combined TGMD-2 and SSA overall skill score' (=0-120). Lastly, subcategory skill scores were also calculated for stability, object control and locomotor skill tasks (e.g., 'overall skill scores' for each of the stability tasks were summed to give a stability skill score).

Concurrent validity: Concurrent validity refers to the extent to which the DC relates to a previously validated movement skills assessment. This was first investigated at an overall level by examining the extent to which the week 1 DC scores related to the 
TGMD-2 and SSA scores. Further, the relationship between week 1 DC score and TGMD-2 skill score was investigated.

The TGMD-2 and SSA were used as the comparison measures for concurrent validity for the following reasons: (i) the validity and reliability for both assessments have been established (Rudd et al., 2015; Ulrich, 2000); (ii) the TGMD-2 has been extensively used as an assessment for movement skill performance; (iii) the SSA provides additional stability tasks that are missing in the TGMD-2, and tasks have been validated to add to the measurement model (Rudd et al., 2015); (iv) the TGMD-2 and SSA have been used in movement skill research in school settings; (v) the TGMD-2 has been validated for children/adolescents of similar age (Issartel et al., 2017); (vi) although the skills in both the TGMD-2 and SSA are completed in isolation by children, the skills assessed within these batteries more closely align with those included in the DC than those used in other movement skill assessments available at the time of study development (no comparative dynamic movement assessments were available); (vii) while TGMD-2 and SSA are considered primarily process-based assessments, there are a selection of product-based criteria (e.g., hop three consecutive times, dribble ball for four consecutive bounces (Ulrich, 2000), log roll for four complete rotations, and back support held for 30 seconds (Rudd et al., 2015)), thus aligning scoring more closely with the DC.

Reliability: Test-re-test reliability was examined by the stability of participants' DC results over the repeated rounds of assessment. The same expert assessor scored each participant on both time-points, and the level of agreement was evaluated.

Inter-rater reliability was explored by investigating how consistent two or more assessors' scores were when observing the same performance. Inter-rater reliability was first assessed at an overall level using the scores given by 3 separate expert assessors on video footage from 12 participants of mixed ability completing the DC. In order to investigate whether amount of training and experience received by assessors influenced reliability, additional analyses examined consistency between expert and newly trained assessor and between expert and trained assessor when scoring DC for 12 and 15 participants, respectively.

Intra-rater reliability was examined by investigating the consistency between scores, when the same trial was scored by the same rater on two separate occasions. Three expert assessors each scored video footage of 12 participants of mixed ability com- 
pleting the DC on two occasions, with a one-week interval between viewings, and levels of agreement between the scores for each assessor was evaluated.

\subsubsection{Statistical Analyses}

Descriptive statistics are presented as mean $\pm \mathrm{SD}$. All statistical tests, with the exception of the confirmatory factor analysis (CFA), were completed using SPSS, version 24 [IBM SPSS Statistics Inc., Chicago, IL, USA]. The CFA was completed using lavaan version 0.6-1 (Rosseel, 2012), in R version 3.5.0 [R Core Team, Vienna, Austria]. In all analyses, data were assessed for violation of the assumptions of normality and statistical significance was set at $\mathrm{p}<0.05$. Participant results were included in each respective analysis if they had sufficient data for the variable concerned.

Surveillance Data and Construct Validity: The proportion of participants successfully demonstrating each DC criterion for the surveillance data was calculated. Two-way ANOVA tests and Chi-squared tests were used to explore the effects of sex and school level on DC scores and on each individual DC task assessment criterion, respectively.

A Principal Component Analysis (PCA) was performed on all DC binary criteria scores. The suitability of each PCA was assessed prior to analysis by inspection of the correlation matrix (each variable required to have at least one correlation with another variable above $r=0.3$ ), further the Kaiser-Meyer-Olkin (KMO) measure needed to be at least 0.6, for sampling adequacy (Kaiser, 1974). In addition, Bartlett's test of sphericity had to achieve statistical significance ( $\mathrm{p}<0.05$ ). To establish DC components, the eigenvalue-one criterion was used (Kaiser, 1960), as well as visual inspection of the scree plot. A Varimax orthogonal rotation was used to aid interpretation, where applicable. A loading of .40 or greater was used to align items onto factors.

Based on the results of the PCA, a CFA was performed to cross-validate the factor structure of the DC. As binary criteria scores were used as indicator variables, weighted least square mean and variance adjusted estimator was used to fit the model. By default, the factor loading of the first indicator of a latent factor was fixed to 1 , thereby fixing the scale of the latent factor. Error terms from the indicator variables were allowed to co-vary within the same factor. Comparative fit index (CFI), TuckerLewis Index (TLI), and root mean square error of approximation (RMSEA) were used to assess model fit, with CFI and TLI of $>0.95$ and RMSEA of $<0.05$, indicating a good fit (Hu \& Bentler, 1999). 
Concurrent Validity: A Pearson's product-moment correlation was used to investigate the strength of relationships between DC, and TGMD-2 and SSA scores and sub-category scores. An $r$ value of, $0-0.19,0.2-0.39,0.4-0.59,0.6-0.79,>0.8$ were interpreted to demonstrate no, low, moderate, moderately-high and high correlation coefficients, respectively (Cohen, 1992).

Reliability: To ascertain evidence for test-retest, inter-rater and intra-rater reliability, intraclass correlation coefficients (ICC), two-way random single measures for absolute agreement (ICC 2,1), with $95 \%$ confidence intervals $(95 \% \mathrm{CI})$, were used to evaluate the level of agreement of week 1 and week 2 scores and of rater scores. A reflect and square root transformation was used where data was non-parametric. For presentation purposes, these variables were transformed for analysis and back transformed. Intraclass correlation coefficients below .50 indicate poor reliability, those between .50 and .75 indicate moderate reliability, and those above .75 indicate good reliability (Portney \& Watkins, 1993). ICC results that indicated moderate reliability $(<.75)$ were further examined using a t-test to investigate if there was a statistically significant mean difference between scores.

\subsection{Results}

Table 11 provides age and sex characteristics of participants that took part in the DC for phase 2 and 3 of the study. On the basis of missing demographic characteristics, 95 participants from the surveillance data were excluded from all construct validity analyses $(n=4260)$, except for the PCA and CFA $(n=4,355)$. 
Table 11. Age (mean $\pm S D)$ and sex (\%) of participants who took part in the Dragon Challenge in study phase 2 and 3

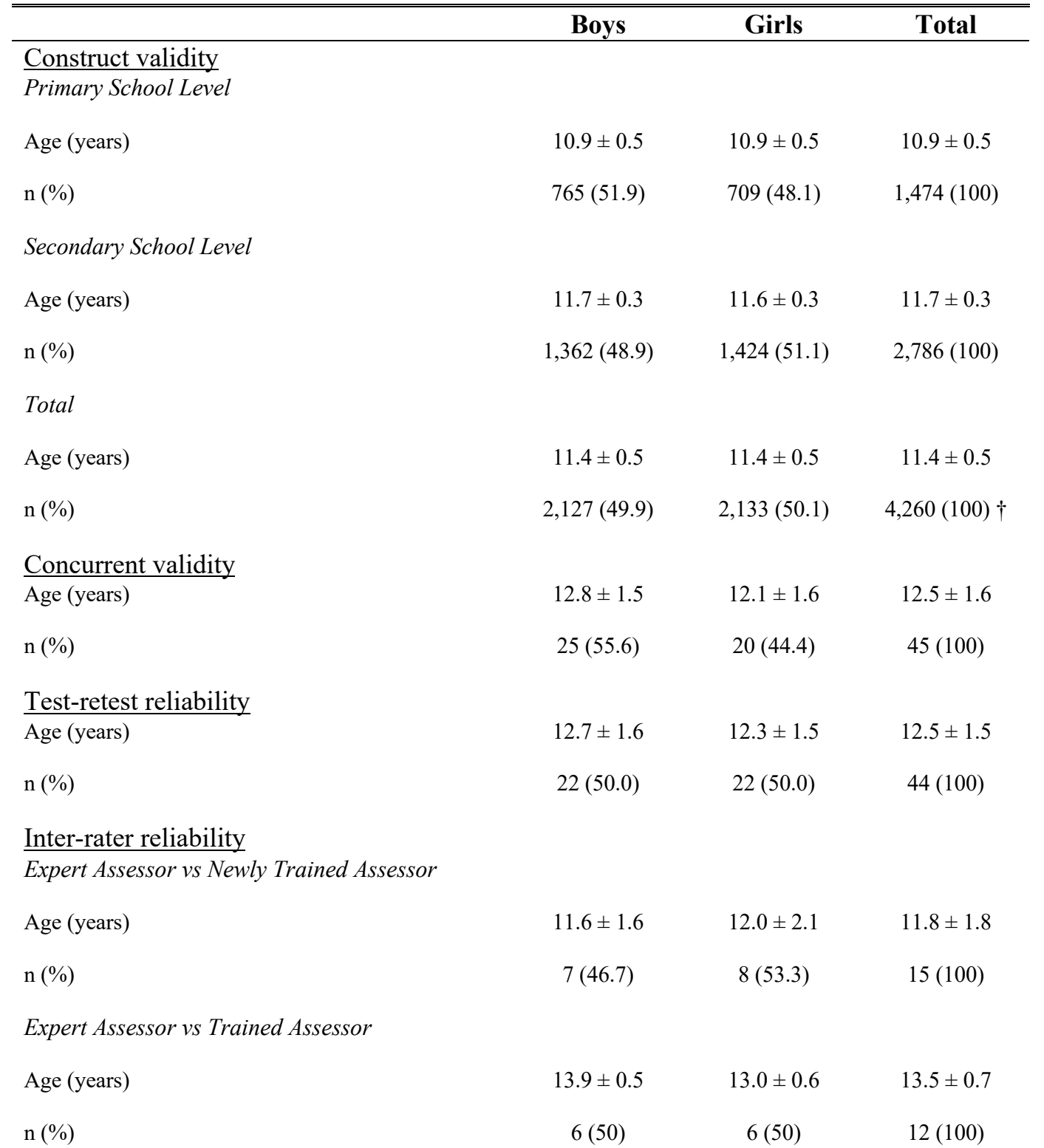

Inter-rater reliability and Intra-rater reliability (video analysis)

$3 x$ Expert Assessors
Age (years)
$11.3 \pm 1.0$
$11.4 \pm 1.1$
$11.3 \pm 1.0$
$\mathrm{n}(\%)$
$6(50)$
$6(50)$
$12(100)$

Note: $\uparrow$ On the basis of missing sex, 95 participants from the surveillance data were excluded from all construct validity analyses, except for the PCA and CFA. For these analyses n=4,355.

Expert assessors: $>50$ hours of DC training and in situ experience; Trained assessor: 20 hours of DC training and in situ experience; Newly trained assessor: 5 hours of DC training. 


\subsubsection{Construct Validity}

Mean scores and standard deviations for DC surveillance data, broken down by sex and school level, are presented in Table 12. There were no statistically significant interactions between sex and school level on DC scores. Therefore, analyses of main effects for each variable were performed. Boys scored higher than girls for all score categories, except stability skills, and secondary school level children scored higher than primary school level children on all score categories apart from time score. The proportion of children who successfully demonstrated each DC criterion, as well as statistically significant sex and school level differences, highlighted by the Chisquared test, are shown in Table 13.

Table 12. Descriptive statistics (mean $\pm S D$ ) for Dragon Challenge (surveillance data) score categories

\begin{tabular}{|c|c|c|c|c|c|c|}
\hline Score Category & $\begin{array}{l}\text { Score } \\
\text { Range }\end{array}$ & $\begin{array}{c}\text { Boys } \\
(n=2,127)\end{array}$ & $\begin{array}{c}\text { Girls } \\
(n=2,133)\end{array}$ & $\begin{array}{l}\text { Primary } \\
(n=1,474)\end{array}$ & $\begin{array}{l}\text { Secondary } \\
(n=2,786)\end{array}$ & $\begin{array}{c}\text { Total } \\
(n=4,260)\end{array}$ \\
\hline DC Total Score & $0-54$ & $33.8 \pm 8.6^{* *}$ & $31.1 \pm 8.3$ & $31.7 \pm 8.3$ & $32.8 \pm 8.6^{* *}$ & $32.4 \pm 8.5$ \\
\hline Technique Score & $0-18$ & $10.9 \pm 3.7^{* *}$ & $9.6 \pm 3.8$ & $9.9 \pm 3.9$ & $10.4 \pm 3.8^{* *}$ & $10.2 \pm 3.8$ \\
\hline Outcome Score & $0-18$ & $11.0 \pm 3.8^{* *}$ & $10.5 \pm 3.6$ & $10.3 \pm 3.6$ & $11.0 \pm 3.7^{* *}$ & $10.8 \pm 3.7$ \\
\hline Time Score & $0-18$ & $11.9 \pm 2.5^{* *}$ & $11.0 \pm 2.6$ & $11.5 \pm 2.4$ & $11.4 \pm 2.7$ & $11.4 \pm 2.6$ \\
\hline Stability Score & $0-12$ & $6.2 \pm 3.3$ & $6.6 \pm 3.3^{* *}$ & $6.1 \pm 3.3$ & $6.6 \pm 3.3^{* *}$ & $6.4 \pm 3.3$ \\
\hline Object Control Score & $0-12$ & $7.6 \pm 3.2^{* *}$ & $5.5 \pm 3.1$ & $6.3 \pm 3.3$ & $6.7 \pm 3.3^{* *}$ & $6.5 \pm 3.3$ \\
\hline Locomotor Score & $0-12$ & $8.1 \pm 2.9$ & $8.0 \pm 2.8$ & $7.8 \pm 2.9$ & $8.2 \pm 2.8^{* *}$ & $8.1 \pm 2.9$ \\
\hline \multicolumn{7}{|c|}{$\begin{array}{l}\text { Note: } \text { Stability skills = sum of technique and outcome criteria } \\
\text { outcome criteria in tasks } 4-6 ; \text { Locomotion skills }=\text { sum of tec } \\
\text { examined using two-way analysis of variance }(\text { ANOVA) test } \\
{ }^{*}=\text { significant sex/school level difference }(\mathrm{p}<0.05) \\
{ }^{* *}=\text { significant sex/school level difference }(\mathrm{p}<0.001) \\
\text { Primary = Primary school-aged children } \\
\text { Secondary = Secondary school-aged children/young people }\end{array}$} \\
\hline
\end{tabular}


Table 13. Proportion (\%) of children successfully demonstrating each Dragon Challenge criterion (surveillance/normative data)

\begin{tabular}{|c|c|c|c|c|c|c|}
\hline \multicolumn{2}{|c|}{ DC Task } & $\begin{array}{c}\text { All } \\
(n=4,260)\end{array}$ & $\begin{array}{c}\text { Boys } \\
(n=2,127) \\
\end{array}$ & $\begin{array}{c}\text { Girls } \\
(n=2,133)\end{array}$ & $\begin{array}{c}\text { Primary } \\
(n=1,474)\end{array}$ & $\begin{array}{c}\text { Secondary } \\
(n=2,786) \\
\end{array}$ \\
\hline \multicolumn{7}{|c|}{ 1.Balance bench } \\
\hline $1.1^{\circ}$ & Moves without hesitation up to turn & 85.5 & 83.8 & $87.1^{*}$ & 84.5 & 86.0 \\
\hline $1.2^{*}$ & Body posture stable & 39.0 & 37.0 & $40.9 *$ & 39.5 & 38.7 \\
\hline $1.3^{\diamond}$ & $\begin{array}{l}\text { Walks length of beam, completes full } \\
\text { turn at } 3 / 4 \text { mark without falling off, } \\
\text { dismounts at end zone }\end{array}$ & 42.7 & 41.7 & 43.6 & 42.7 & 42.6 \\
\hline \multicolumn{7}{|c|}{ 2. Core Agility } \\
\hline $2.1^{\circ}$ & $\begin{array}{l}\text { Hands \& legs extended \& held with } \\
\text { tension, with shoulders \& feet off the } \\
\text { floor }\end{array}$ & 37.3 & 31.4 & $43.2^{* *}$ & 33.0 & $39.6^{* *}$ \\
\hline $2.2^{*}$ & $\begin{array}{l}\text { Controlled \& fluent transition through } \\
\text { shapes }\end{array}$ & 41.1 & 37.9 & $44.3^{* *}$ & 41.0 & 41.1 \\
\hline $2.3^{\diamond}$ & $\begin{array}{l}\text { Completes } 4 \text { positions in correct order, } \\
\text { rotating both ways }\end{array}$ & 75.4 & 71.8 & $78.9^{* *}$ & 70.5 & $77.9^{* *}$ \\
\hline \multicolumn{7}{|c|}{ 3. Wobble Spot } \\
\hline $3.1^{\circ}$ & $\begin{array}{l}\text { Non-support foot does not touch support } \\
\text { leg/foot/wobble spot/floor }\end{array}$ & 50.5 & 50.3 & 50.8 & 46.0 & $52.9^{* *}$ \\
\hline $3.2^{*}$ & Body \& head are stable/still & 48.9 & 48.3 & 49.5 & 44.5 & $51.3^{* *}$ \\
\hline $3.3^{\diamond}$ & $\begin{array}{l}\text { Completes } 5 \text { bean bag passes around } \\
\text { body whilst balancing on wobble spot on } \\
\text { one leg }\end{array}$ & 50.8 & 50.5 & 51.1 & 46.3 & $53.2 * *$ \\
\hline \multicolumn{7}{|c|}{ 4. Overarm throw } \\
\hline $4.1^{\circ}$ & $\begin{array}{l}\text { Throwing arm moves in a backward arc } \\
\text { to initiate throw }\end{array}$ & 57.6 & $73.2 * *$ & 42.0 & 56.2 & 58.3 \\
\hline $4.2^{*}$ & $\begin{array}{l}\text { Steps with the foot opposite throwing } \\
\text { hand towards target }\end{array}$ & 73.1 & $86.6^{* *}$ & 59.7 & 71.7 & 73.9 \\
\hline $4.3^{\diamond}$ & Overarm throw directly hits target & 47.9 & $53.5^{* *}$ & 42.3 & 44.2 & $49.8^{*}$ \\
\hline \multicolumn{7}{|c|}{ 5. Basketball Dribble } \\
\hline $5.1^{\circ}$ & Pushes ball with fingertips & 61.4 & $75.7^{* *}$ & 47.1 & 57.7 & $63.3 * *$ \\
\hline $5.2^{\circ}$ & Controlled directional dribbling & 71.1 & $77.2^{* *}$ & 65.0 & 67.1 & $73.2^{* *}$ \\
\hline $5.3^{\diamond}$ & $\begin{array}{l}\text { Dribbles around all spots using either } \\
\text { hand. Cannot catch ball/use two hands } \\
\text { simultaneously }\end{array}$ & 64.2 & $69.9^{* *}$ & 58.5 & 62.4 & 65.1 \\
\hline \multicolumn{7}{|c|}{ 6. Catch } \\
\hline $6.1^{\circ}$ & Feet move in line with rebound & 62.9 & $73.4 * *$ & 52.5 & 60.8 & $64.1^{*}$ \\
\hline $6.2^{*}$ & Catches ball with hands only & 32.3 & $40.7^{* *}$ & 24.0 & 31.0 & 33.0 \\
\hline $6.3^{\diamond}$ & Successful catch off rebound net & 35.6 & $44.4^{* *}$ & 26.9 & 34.8 & 36.0 \\
\hline \multicolumn{7}{|c|}{ 7. T-Agility } \\
\hline $7.1^{\circ}$ & Plants \& drives off outside foot & 29.6 & $33.3^{* *}$ & 25.9 & 27.8 & 30.5 \\
\hline $7.2^{\circ}$ & Side-stepping on balls of feet & 50.0 & 51.2 & 48.9 & 45.8 & $52.3 * *$ \\
\hline $7.3^{\diamond}$ & $\begin{array}{l}\text { Moves through all points of ' } \mathrm{T} \text { ' facing } \\
\text { forwards }\end{array}$ & 58.6 & 59.5 & 57.8 & 52.9 & $61.7^{* *}$ \\
\hline \multicolumn{7}{|c|}{ 8. Jumping Patterns } \\
\hline $8.1^{\circ}$ & Arms drive over first hurdle & 72.2 & 71.9 & 72.5 & 72.9 & 71.9 \\
\hline $8.2^{\circ}$ & Rhythmical pattern throughout & 64.2 & 62.2 & $66.1^{*}$ & 60.0 & $66.4^{* *}$ \\
\hline $8.3^{\diamond}$ & $\begin{array}{l}\text { Completes jumping pattern sequence } \\
\text { correctly. No contact with hurdles }\end{array}$ & 65.5 & 62.4 & $68.6^{* *}$ & 63.7 & 66.5 \\
\hline \multicolumn{7}{|c|}{ 9. Sprint } \\
\hline $9.1^{\circ}$ & Drives off balls of feet, leaning forwards & 70.2 & $74.2^{* *}$ & 66.3 & 69.8 & 70.5 \\
\hline $9.2^{*}$ & Arms bent, driving forward \& backwards & 76.6 & $79.1^{* *}$ & 74.1 & 78.3 & 75.7 \\
\hline $9.3^{\diamond}$ & $\begin{array}{l}\text { Runs through start gate \& then through to } \\
\text { finish }\end{array}$ & 97.0 & 97.4 & 96.6 & 97.0 & 97.0 \\
\hline $\begin{array}{l}\text { Note. } \\
\diamond=\mathrm{pr} \\
:=\mathrm{pr} \\
*=\mathrm{sig} \\
* *=\mathrm{s} \\
\text { Prima } \\
\text { Secon }\end{array}$ & $\begin{array}{l}\text { duct/outcome characteristic/quality indicator } \\
\text { cess/technique characteristic/quality indicato } \\
\text { ificant sex/school level difference }(\mathrm{p}<0.05) \\
\text { snificant sex/school level difference }(\mathrm{p}<0.0 \\
=\text { Primary school-aged children } \\
\text { ary = Secondary school-aged children/young }\end{array}$ & $\begin{array}{l}\text { outcome of mo } \\
\text { (technique or } n \\
\text { 1) } \\
\text { people }\end{array}$ & $\begin{array}{l}\text { nent) } \\
\text { ement form) }\end{array}$ & & & \\
\hline
\end{tabular}


PCA on DC criteria scores: PCA was found to be suitable according to the correlation matrix, overall KMO (.76) and Bartlett's test of sphericity $(\mathrm{p}<0.001)$. The PCA revealed nine components that had eigenvalues greater than one, 5.11, 2.53, $2.01,1.83,1.54,1.42,1.37,1.19,1.15$, and which explained $18.94 \%, 9.39 \%, 7.46 \%$, $6.76 \%, 5.71 \%, 5.24 \%, 5.09 \%, 4.40 \%, 4.26 \%$, respectively. Visual inspection of the scree plot also indicated that nine factors should be retained. This nine-component solution explained $67.24 \%$ of the total variance and the rotated solution exhibited a simple structure. The interpretation of the data was consistent with the skill combinations the DC was designed to measure, with strong loadings of balance bench criteria scores on component one, core agility criteria scores on component two, wobble spot criteria scores on component three, overarm throw criteria scores on component four, basketball dribble criteria scores on component five, catch criteria scores on component six, t-agility criteria scores on component seven, jumping patterns criteria scores on component eight, sprint criteria scores on component nine. Component loadings of the rotated solution (Table 14) were all $>0.4$. 
Table 14. Rotated Component Matrix from Principal Component Analysis on Dragon Challenge criteria score

\begin{tabular}{|c|c|c|c|c|c|c|c|c|c|}
\hline \multirow{2}{*}{ DC Task Criteria } & \multicolumn{9}{|c|}{ Component } \\
\hline & 1 & 2 & 3 & 4 & 5 & 6 & 7 & 8 & 9 \\
\hline Balance Bench Technique 1 & .473 & .018 & .103 & .010 & .094 & .009 & .022 & .178 & .136 \\
\hline Balance Bench Technique 2 & .951 & .075 & .057 & .043 & .008 & .031 & .043 & .003 & .021 \\
\hline Balance Bench Outcome & .952 & .061 & .065 & .040 & .025 & .042 & .041 & .016 & .020 \\
\hline Core Agility Technique 1 & .088 & .744 & .079 & .028 & .022 & .002 & .084 & .107 & .092 \\
\hline Core Agility Technique 2 & .062 & .798 & .055 & .042 & .075 & .037 & .022 & .096 & .052 \\
\hline Core Agility Outcome & -.006 & .661 & .035 & -.007 & .082 & .055 & .089 & .057 & .044 \\
\hline Wobble Spot Technique 1 & .088 & .070 & .974 & .050 & .056 & .053 & .048 & .094 & .062 \\
\hline Wobble Spot Technique 2 & .087 & .075 & .963 & .048 & .056 & .045 & .043 & .101 & .066 \\
\hline Wobble Spot Outcome & .087 & .068 & .977 & .051 & .051 & .049 & .046 & .095 & .063 \\
\hline Overarm Throw Technique 1 & -.023 & -.015 & .044 & .755 & .152 & .076 & .134 & .044 & .140 \\
\hline Overarm Throw Technique 2 & .003 & .019 & .026 & .764 & .084 & .101 & .003 & .009 & .024 \\
\hline Overarm Throw Outcome & .074 & .039 & .038 & .544 & .058 & .039 & .012 & .072 & -.004 \\
\hline Basketball Dribble Technique 1 & .021 & .026 & .032 & .243 & .674 & .164 & .058 & .039 & .102 \\
\hline Basketball Dribble Technique 2 & .070 & .113 & .045 & .102 & .857 & .109 & .079 & .085 & .039 \\
\hline Basketball Dribble Outcome & .050 & .074 & .068 & .029 & .800 & .067 & .076 & .087 & .083 \\
\hline Catch Technique 1 & .006 & .074 & .055 & .142 & .182 & .676 & .126 & .058 & .033 \\
\hline Catch Technique 2 & .043 & .018 & .039 & .060 & .077 & .931 & .037 & .051 & .044 \\
\hline Catch Outcome & .035 & .022 & .040 & .059 & .075 & .945 & .030 & .035 & .044 \\
\hline T-Agility Technique 1 & .030 & .147 & .044 & .190 & .075 & .117 & .608 & .151 & .160 \\
\hline T-Agility Technique 2 & .046 & .060 & .042 & .012 & .065 & .034 & .819 & .045 & .060 \\
\hline T-Agility Outcome & .028 & .036 & .034 & -.011 & .066 & .046 & .822 & .061 & -.011 \\
\hline Jumping Patterns Technique 1 & .078 & .102 & .059 & .102 & .081 & .030 & .125 & .430 & .350 \\
\hline Jumping Patterns Technique 2 & .076 & .127 & .132 & .068 & .097 & .075 & .093 & .851 & .059 \\
\hline Jumping Patterns Outcome & .082 & .116 & .098 & .038 & .065 & .052 & .084 & .871 & .052 \\
\hline Sprint Technique 1 & .045 & .049 & .073 & .175 & .106 & .061 & .116 & .201 & .689 \\
\hline Sprint Technique 2 & .050 & .053 & .048 & .103 & .064 & .032 & .049 & .122 & .768 \\
\hline Sprint Outcome & .053 & .062 & .028 & -.091 & .026 & .017 & -.001 & -.073 & .604 \\
\hline
\end{tabular}

Note. Extraction Method: Principal Component Analysis. Rotation Method: Varimax with Kaiser

Normalization. Rotation converged in 6 iterations.

CFA on the DC criteria scores: Based on the PCA results, CFA was conducted to confirm the nine-factor structure, as well as to examine whether the nine latent factors loaded onto a higher order factor (physical competence). Following the addition of three correlations between error terms within the same factor, the fit for the hypothesised model (Figure 5), was good (CFI, 1.00; TLI, 1.00; RMSEA, 0.038; $90 \%$ 
confidence interval 0.037 - 0.040). Factor loadings ranged from $0.45-0.99$, showing that the factor validity was acceptable to excellent.

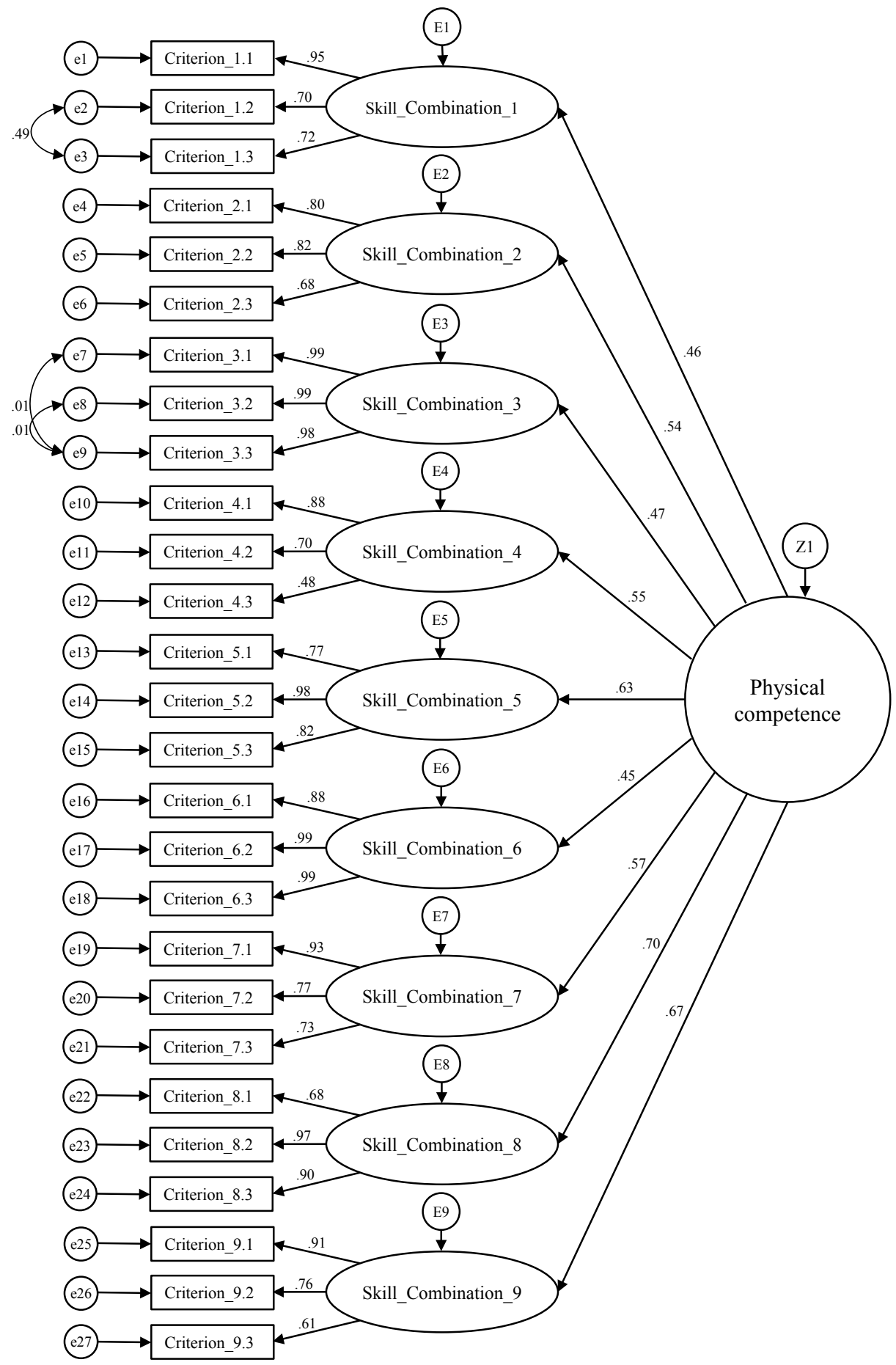

Figure 5. Factor structure of the Dragon Challenge 


\subsubsection{Concurrent Validity}

Results for concurrent validity show that there was a significant high positive correlation between DC total score ( $35.9 \pm 8.5)$ and "combined TGMD-2 and SSA overall skill score' $\left(72.5 \pm 10.9 ; r(43)=.86, r^{2}=.74, \mathrm{p}<0.001\right)$. Relationships for subcategory scores between DC and TGMD-2 and SSA skills scores, across stability tasks $(7.2 \pm 3.2,7.8 \pm 3.7 ; r(43)=.46, \mathrm{p}=0.001)$, object control tasks $(8.0 \pm 3.4$, $32.5 \pm 6.9 ; r(43)=.83, \mathrm{p}<0.001)$ and locomotor tasks $(8.5 \pm 2.5,32.2 \pm 3.4 ; r(43)$ $=.60, \mathrm{p}<0.001)$, showed significant moderate to high positive correlations. Finally, there was a significant high positive correlation between DC score $(35.93 \pm 8.54)$ and TGMD-2 'overall skill score' $\left(64.71 \pm 8.66 ; r(43)=.81, r^{2}=.66, \mathrm{p}<0.001\right)$.

\subsubsection{Reliability}

Test-Retest Reliability: The DC total score showed good test-retest reliability across the one-week interval (ICC $=0.80 ; 95 \%$ CI: 0.63, 0.90; $p<0.001$ ). Evidence for test-retest reliability was good for technique scores (ICC $=0.77$; 95\%CI: $0.58,0.88$; $\mathrm{p}<0.001$ ), and high-moderate for time scores (ICC $=0.74 ; 95 \%$ CI: $0.57,0.85 ; \mathrm{p}<$ 0.001 ) and for outcome scores (ICC $=0.71 ; 95 \% \mathrm{CI}$ : 0.52, 0.83; $\mathrm{p}<0.001$ ). Follow up t-tests revealed no significant mean difference in time score between test $(12.18$ points) and retest (12.93 points) scores $(t=.837, \mathrm{p}=0.41)$ and no statistically significant mean difference in outcome score between the test (11.95 points) and retest (12.00 points) scores $(t=.103, \mathrm{p}=0.92)$.

Further, test-retest reliability for skill sub-categories was good for object control skills score (ICC $=0.80 ; 95 \%$ CI: $0.67,0.89 ; \mathrm{p}<0.001$ ), high-moderate for locomotor skills score (ICC $=0.68 ; 95 \%$ CI: $0.49,0.81 ; \mathrm{p}<0.001)$, and moderate for stability skills score $(\mathrm{ICC}=0.60 ; 95 \% \mathrm{CI}: 0.38,0.76 ; \mathrm{p}<0.001)$. No significant mean difference was found in locomotor skills score between test ( 8.43 points) and retest ( 8.59 points) scores $(t=.525, \mathrm{p}=0.60)$, nor in stability skills score between test (7.14 points) and retest (6.61 points) scores $(t=-1.25, \mathrm{p}=0.22)$.

Inter-Rater and Intra-Rater Reliability: Inter-rater and intra-rater reliability on all comparison levels (Table 15) showed significant relationships and were classed as good (all ICCs $>.85$ ). 
Table 15. Inter- and intra-rater reliability results for Dragon Challenge scores and sub-category scores

\begin{tabular}{|c|c|c|c|c|}
\hline \multirow[b]{2}{*}{ Reliability Test } & \multirow{2}{*}{$\begin{array}{c}\begin{array}{c}\text { Intraclass } \\
\text { Correlation }\end{array} \\
\text { ICC }\end{array}$} & \multicolumn{2}{|c|}{$95 \% \mathrm{CI}$} & \multirow[t]{2}{*}{ Strength of ICC } \\
\hline & & Lower & Upper & \\
\hline \multicolumn{5}{|l|}{ Inter-rater Reliability } \\
\hline \multicolumn{5}{|c|}{ Expert Assessor vs. Newly Trained Assessor } \\
\hline Dragon Challenge Total Score & $.950 * *$ & .859 & .983 & Good \\
\hline Technique Score & $.839 * *$ & .592 & .942 & Good \\
\hline Outcome Score & $.916 * *$ & .742 & .972 & Good \\
\hline Time Score & - & - & - & - \\
\hline Stability Skills Score & $.787 * *$ & .479 & .923 & Good \\
\hline Object Control Skills Score & $.945 * *$ & .847 & .981 & Good \\
\hline Locomotor Skills Score & $.903 * *$ & .742 & .966 & Good \\
\hline \multicolumn{5}{|c|}{ Expert Assessor vs. Trained Assessor } \\
\hline Dragon Challenge Total Score & $.986 * *$ & .951 & .996 & Good \\
\hline Technique Score & $.987 * *$ & .957 & .996 & Good \\
\hline Outcome Score & $.920 * *$ & .647 & .979 & Good \\
\hline Time Score & - & - & - & - \\
\hline Stability Skills Score & $.941 * *$ & .792 & .983 & Good \\
\hline Object Control Skills Score & $.972 * *$ & .907 & .992 & Good \\
\hline Locomotor Skills Score & $.964 * *$ & .882 & .990 & Good \\
\hline \multicolumn{5}{|c|}{3 x Expert Assessors } \\
\hline Dragon Challenge Total Score & $.942 * *$ & .837 & .982 & Good \\
\hline Technique Score & $.889 * *$ & .718 & .964 & Good \\
\hline Outcome Score & $.899 * *$ & .758 & 967 & Good \\
\hline Time Score & $.990 * *$ & .973 & .997 & Good \\
\hline Stability Skills Score & $.850 * *$ & .666 & .949 & Good \\
\hline Object Control Skills Score & $.918 * *$ & .803 & .973 & Good \\
\hline Locomotor Skills Score & $.904 * *$ & .753 & .969 & Good \\
\hline \multicolumn{5}{|l|}{ Intra-rater Reliability } \\
\hline \multicolumn{5}{|c|}{ Dragon Challenge Total Score } \\
\hline Expert Assessor 1 & 1.000 & - & _- & Good \\
\hline Expert Assessor 2 & $.990 * *$ & .967 & .997 & Good \\
\hline Expert Assessor 3 & $.999 * *$ & 997 & 1.00 & Good \\
\hline \multicolumn{5}{|c|}{ Technique Score } \\
\hline Expert Assessor 1 & 1.000 & - & - & Good \\
\hline Expert Assessor 2 & $.977 * *$ & .904 & .994 & Good \\
\hline Expert Assessor 3 & $.995 * *$ & 984 & .999 & Good \\
\hline \multicolumn{5}{|c|}{ Outcome Score } \\
\hline Expert Assessor 1 & 1.000 & - & - & Good \\
\hline Expert Assessor 2 & $.947 * *$ & .830 & .984 & Good \\
\hline Expert Assessor 3 & $.989 * *$ & .965 & .997 & Good \\
\hline \multicolumn{5}{|c|}{ Time Score } \\
\hline Expert Assessor 1 & 1.000 & - & - & Good \\
\hline Expert Assessor 2 & 1.000 & - & - & Good \\
\hline Expert Assessor 3 & $.991 * *$ & .968 & .997 & Good \\
\hline \multicolumn{5}{|c|}{ Stability Skills Score } \\
\hline Expert Assessor 1 & 1.000 & - & - & Good \\
\hline Expert Assessor 2 & $.963 * *$ & .878 & .989 & Good \\
\hline Expert Assessor 3 & $.997 * *$ & .991 & .999 & Good \\
\hline \multicolumn{5}{|c|}{ Object Control Skills Score } \\
\hline Expert Assessor 1 & 1.000 & - & - & Good \\
\hline Expert Assessor 2 & $.987 * *$ & .955 & .996 & Good \\
\hline Expert Assessor 3 & $.991 * *$ & .969 & .997 & Good \\
\hline \multicolumn{5}{|c|}{ Locomotor Skills Score } \\
\hline Expert Assessor 1 & 1.000 & - & - & Good \\
\hline Expert Assessor 2 & $.962 * *$ & .867 & .989 & Good \\
\hline Expert Assessor 3 & $.975 * *$ & .916 & .993 & Good \\
\hline
\end{tabular}




\subsection{Discussion}

Many current measures that inform physical competency as part of physical literacy assessments (Giblin et al., 2014; Robinson \& Randall, 2017), in children and adolescents (Bruininks \& Bruininks, 2005; Cairney et al., 2018; Henderson et al., 2007; Physical and Health Education Canada (PHE Canada), 2014; Ulrich, 2000), use isolated movement skills. Assessing discrete movement skills in isolation fails to account for the utilisation of combined and complex movement skills observed during physical activity and play, and needed to demonstrate physical competence and physical literacy (Whitehead, 2010). This study therefore aimed to develop the DC, a land-based dynamic measure of movement capacities/skills and movement patterns to assess elements of physical competence for 10-14 year olds.

The DC consists of nine tasks completed in a timed circuit, incorporating the utilisation of fundamental, combined and complex movement skills/capacities, to produce refined/complex and specific movement patterns. The DC can be used for assessment for learning (summative and/or formative), and as a national surveillance tool, that can be aligned to physical literacy programmes and physical education curriculum. The assessment criteria for the DC includes both technique (process) and outcome (product) indicators of movement performance, to provide a more complete picture of physical competence levels than currently used assessments that include primarily product- or process-based criteria (Bruininks \& Bruininks, 2005; Cairney et al., 2018; Henderson et al., 2007; Physical and Health Education Canada (PHE Canada), 2014; Ulrich, 2000). Given that the DC is completed in a continuous circuit, tasks are dynamic, sequential and include additional layers of complexity in a more open 'authentic' environment than many existing measures that assess skills in isolation (Bruininks \& Bruininks, 2005; Cairney et al., 2018; Henderson et al., 2007; Physical and Health Education Canada (PHE Canada), 2014; Ulrich, 2000). The DC is internally paced by the participants, whom are required to perform the tasks competently as fast as they can, thereby requiring a speed-accuracy trade-off. Although not directly measured, children also need to apply awareness of space, effort, and relationships to objects, goals, and boundaries to complete the challenge. Thus, within the DC, children are required to demonstrate movement concepts and attributes expected to be displayed by a physically competent child, for example, "movement with poise, economy and confidence in a wide variety of challenging situations" and "sensitive perception in 'reading' all aspects of the physical environment, anticipating movement needs or possibilities and responding appropriately to these, with intelligence and imagination" (Whitehead, 2010). Therefore, given the paucity of dynamic mea- 
sures of movement skills/capacities and varying complexities of movement patterns to inform physical competence in children aged 10-14 years, this study fills a critical gap in the current literature in this field.

\subsubsection{Construct Validity}

Boys obtained significantly higher DC total, time, technique and outcome scores (Table 12). When broken down into sub-categories for comparison purposes, boys scored significantly higher than girls for tasks primarily utilising object control skills, with more detailed analysis (Table 13) showing that significantly more boys demonstrated proficiency at each of the assessment criteria for the overarm throw, basketball dribble and catch. These sex differences seem to be in line with numerous studies that have shown that boys outperform girls at object control skills (Barnett, van Beurden, Morgan, Brooks, \& Beard, 2010; Cairney et al., 2018; Hume et al., 2008). On the other hand, girls scored significantly higher than boys for tasks primarily utilising stability skills, with significantly more girls demonstrating proficiency at each of the assessment criteria for core agility, as well as two of the assessment criteria for balance bench (criterion 1.1, 1.2; Table 13). While literature regarding sex differences in stability skills is less prevalent, young girls have been shown to display greater aptitude in process-oriented balancing skills (Pienaar, van Reenen, \& Weber, 2017). In line with many studies that report no sex difference in locomotor skills (Barnett et al., 2010; Cairney et al., 2018; Hume et al., 2008), no significant difference was found in score between boys and girls for the locomotor skills sub-category. Moreover, girls typically excel at hopping and skipping in comparison to boys (Duger, Bumin, Uyanik, Aki, \& Kayihan, 1999), supporting our findings that significantly more girls were proficient in two of the jumping patterns criteria (criteria 8.2 and 8.3). Considering these findings within the context of sex differences, the DC data are aligned to current literature on physical competence and movement skill competence.

Not only did secondary school level children obtain significantly higher DC total, technique and outcome scores compared to primary school level children, but they also scored significantly higher for object control skills, locomotor skills, and stability skills. Given that gross motor skill is developmental by age and stage, these results are standard within the literature (Gallahue et al., 2012). It is worth noting, however, that there was no significant difference in time score between primary and secondary school children. This was unexpected as previous studies have shown that running speed increases with age in children (Duger et al., 1999), although this discrepancy may be explained by the speed-accuracy trade-off made by children when completing 
the DC. Thus, the higher accuracy of the secondary school level children at the DC tasks would have resulted in them taking longer to complete the tasks than the less accurate primary school level children. In summary, the findings in relation to sex and age differences are consistent with the literature.

Since the factor structure showed good model fit (Figure 5), it is reasonable to conclude that, unlike existing measures of physical competence (NSW Department of Education and Training, 2000; Rudd et al., 2015; Ulrich, 2000), the DC does not measure movement skills in isolated skill categories (i.e., stability, object control, locomotor skills), but rather requires the application of different combinations of movement skills for each task. Thus, the good fit of the model adds support to the design of the DC, as each task was selected to include the utilisation of skills from multiple movement categories to produce a series of movement patterns, and to the contention that the DC includes combined and complex movement skills. Additionally, the adequate factor loadings of each criterion scores onto its respective latent factor suggests that each criterion score is a good indicator, giving strength to the choice of criteria in the DC scoring system. Finally, as each of the nine first order latent factors (skill combinations) loaded onto a higher order latent factor (physical competence), it suggests that the combination of skills required by each DC task is needed for children to be physically competent. It must be noted, however, that physical competence is a multidimensional concept, therefore there are additional aspects of physical competence that are not represented in this model, for example, combinations of movement skills in different settings (water, air, ice), or movement forms (Jurbala, 2015; Whitehead, 2010).

\subsubsection{Concurrent Validity}

The DC total score showed a significant high positive relationship with the "combined TGMD-2 and SSA overall skill score' and with 'TGMD-2 overall skill score', demonstrating strong concurrent validity between the assessments. When broken down across sub-categories, there was a significant high relationship between object control task scores in the DC and TGMD-2, whereas the DC stability and locomotor task scores showed only significant moderate relationships with those included in the TGMD-2 and SSA. While the stability and locomotor skills in the two assessments were matched for comparison purposes, the tasks required by the TGMD-2 and SSA compared to the DC were not identical. Moreover, as evidenced by the CFA on DC criteria scores, each of the DC tasks require a unique combination of movement skills/capacities to perform the refined/complex and specific movement patterns. 
Therefore, the differences in stability and locomotor tasks in the TGMD-2 and SSA compared to the DC probably contributed to lowering the correlation of these subcategories. Nevertheless, all relationships, both in total scores and in subcategory scores, between the tools were significant moderate to strong, indicating that the DC ranks children in similar order to previously-validated tools.

\subsubsection{Reliability}

Test-retest reliability for both DC total and technique scores, was good across a oneweek interval. However, time and outcome scores only showed high-moderate and moderate test-retest reliability, respectively. This may also be reflective of the speedaccuracy trade-off associated with the DC assessment tasks, with children perhaps making different decisions as to which to prioritise when performing the DC on multiple occasions. Upon further investigation, there was no significant difference in mean outcome or time scores between the test and retest, providing support that no learning effect was present. Since DC total score showed good test-retest reliability over a one-week interval, and all other scores showed moderate-to-good test-retest reliability, with statistics at least as strong as those for other measurement tools (Bruininks \& Bruininks, 2005; Henderson et al., 2007; Longmuir et al., 2017; Ulrich, 2000; Wiart $\&$ Darrah, 2001), then the tenet that the DC is a stable measure is supported.

Inter-rater reliability was good for each of the DC total, time, technique, outcome, and sub-category scores when comparing three separate expert standard assessors' score. These levels of inter-rater reliability are similar to those of the TGMD-2, BOT-2 and MABC-2, but stronger than those of the CAMSA measurement tool (Bruininks \& Bruininks, 2005; Henderson et al., 2007; Longmuir et al., 2017; Ulrich, 2000; Wiart \& Darrah, 2001). Inter-rater reliability was also good for the DC total, technique, outcome, and subcategory scores, both when comparing the level of agreement of expert assessor's scores and newly-trained assessor's scores and when comparing the level of agreement of expert assessor's scores and trained assessor's scores. There was stronger reliability between the expert assessor and trained assessor than between the expert assessor and newly-trained assessor in all scores. This suggests that the additional field time that the trained assessor undertook compared to the newly-trained assessor may have resulted in more reliable assessments. Taken together, the interrater reliability results may imply that only one skilled assessor is needed to achieve a reliable assessment of participants taking part in the DC. Moreover, each of the three expert assessors' DC total, time, technique, outcome, and sub-category scores showed good intra-rater reliability, consistent with the levels of intra-rater reliability 
of other measurement tools (Bruininks \& Bruininks, 2005; Henderson et al., 2007; Longmuir et al., 2017; Ulrich, 2000; Wiart \& Darrah, 2001), suggesting that the current DC assessment criteria are sufficiently clear to allow an accurate assessment of a participant in one viewing.

\subsubsection{Feasibility}

There are currently no guidelines for determining the optimal duration of an assessment tool, therefore the purpose, information yielded, and time for completion should all be considered when examining assessment feasibility. Assessing a child in the DC required at least one assessor and administrator, with an additional assistant to supervise the non-participating children, in line with most other assessments (Bruininks \& Bruininks, 2005; Henderson et al., 2007; Longmuir et al., 2017; Ulrich, 2000). While this may seem burdensome, the balance between developing sufficient data for surveillance and adequate detailed insight to provide feedback and promote learning was achieved using this approach.

Children typically only took between 90 and 240 seconds to complete the DC, and an overall estimated assessment time of 10 minutes per child. Large sports halls can facilitate multiple concurrent DC circuits, thus decreasing time to assess larger numbers of children. However, the trade-off is that more assessors and administrators are required with multiple set-ups. In many previously-validated movement skill assessments (Bruininks \& Bruininks, 2005; Cairney et al., 2018; Henderson et al., 2007; Physical and Health Education Canada (PHE Canada), 2014; Ulrich, 2000; Wiart \& Darrah, 2001), an average of 15-60 minute assessment time per child was required. Although some of these assessments were initially created for differing circumstances (e.g., developmental coordination disorder), they have all been used to assess the physical aspects of physical literacy, in an educational setting (Giblin et al., 2014; Robinson \& Randall, 2017). In comparison to these assessments, the DC assessment time per child is considerably less, providing evidence that the DC is a time-efficient measure. Conversely, the CAMSA (Longmuir et al., 2017), requires less time to complete (set up time $=5-7$ mins; assessment time $=25$ min for 20 children) than the DC. This is due, at least in part, to the incorporation of more tasks and indeed performance criteria in the DC. It is therefore postulated that longer assessment times to yield more information are reasonable.

The DC produced important information on a child's movement skills/capacities and varying complexities of movement patterns to inform physical competence and phys- 
ical literacy, and so, as in other assessments within schools (English, Maths and Science exams), time and effort needs to be applied for progressive learning. The decreased assessment time associated with the DC compared to the many previouslyvalidated assessments (Bruininks \& Bruininks, 2005; Cairney et al., 2018; Henderson et al., 2007; Physical and Health Education Canada (PHE Canada), 2014; Ulrich, 2000; Wiart \& Darrah, 2001), increases its feasibility as a population-level surveillance tool. Furthermore, in this study we have demonstrated that we can collect data on a national sample of children $(n=4,355)$, supporting our premise that DC can be used as an assessment for learning and a national surveillance tool.

\subsubsection{Limitations and Future Directions}

It is important to note that whilst, in comparison to many other existing assessments, the DC is more inclusive of the constructs of Whitehead's interpretations of physical competence (Whitehead, 2010), it does not provide a complete assessment of physical competence. Specifically, the DC does not reflect physical competence in terms of different varieties of contexts and durations of activities, activity settings (i.e., water, air, ice) (Jurbala, 2015; Whitehead, 2010), or different movement forms (i.e., adventure, aesthetic, athletic, competitive, fitness and health, interactional/relational) (Whitehead, 2010). However, many land-based measures assume the transferability of movement capacities/skills and movement patterns assessed in the measures, to other contexts (Giblin et al., 2014; Robinson \& Randall, 2017). This may also be the case for the DC, but future studies may wish to investigate the use of the DC to predict the participation in differing movement forms and activity settings. The authors of this study also acknowledge that although the DC generally showed good concurrent validity with the TGMD-2 and SSA, a gold standard measure that is more dynamic and includes more aspects of combined and complex movement skills, rather than individual skills in isolation, may be more appropriate for comparisons. However, at the time of study design there was no gold standard assessment that assessed such movement skills. Furthermore, as a compromise for being able to use the DC at a population-level, some criterion that were typically considered critical movement features (e.g., hip then shoulder rotation for the overarm throw), were not incorporated into the assessment criteria due to the difficulty of observation in real-time during protocol development.

Although discriminant and clinical use of the DC was not a planned outcome in the current study, further analysis of the surveillance data $(n=4,355)$, reported in a separate DC surveillance report, found that the DC was able to significantly differentiate 
between children with and without an additional or special learning needs, across all DC scores (Stratton, Foweather, \& Hughes, 2017). However, additional investigations are required to develop the DC so that is fully inclusive, irrespective of disability. Moreover, the high percentage of success for both boys and girls on criterion 9.3 (Table 13), suggests that a ceiling effect may be present for this product criterion. Therefore, an adjustment of this criterion, perhaps with the use of Rasch analysis (Zhu $\&$ Cole, 1996), may be warranted. Finally, since the tasks included in the DC were selected to be a developmentally-appropriate assessment of physical competence for children in developed countries with similar physical education curricular and sport programmes, future studies should examine cultural differences to evaluate whether the tasks chosen are also valid in jurisdictions with different physical education and sport programmes.

\subsection{Conclusion}

The DC was designed as a tool to measure elements of physical competence, representing a more ecological measurement of fundamental, combined, and complex movement skills in one assessment. These skills are combined in the DC to form complex movement patterns in a more authentic environment and can be measured in a time-efficient manner. The DC is novel in that it offers a dynamic land-based measure to inform physical competence for formative and summative assessment purposes, as well as for national surveillance, with accurate data collected from a national sample of over 4,300 children in Wales. Our results demonstrate that the DC is a valid and reliable measure in children aged 10-14 years. Further investigation into the potential of the DC to reflect physical competence in terms of different contexts, durations, and activity settings, as well as the development of measures of the remaining physical literacy domains, should be of focus to construct a full physical literacy measurement model.

\subsection{Summary: Study 4}

The first aim of this chapter was to develop a dynamic measure of physical competence that requires a participant to demonstrate fundamental, combined and complex movement skills, and assessors to score both processes and products (Dragon Challenge; DC). The second aim was to assess the psychometric properties of the DC in 10-14 year old children.

In support of construct validity, boys $(\mathrm{p}<0.001)$ and secondary school children $(\mathrm{p}<$ 
0.001) obtained higher DC total scores than girls and primary school children, respectively. A principal component analysis revealed a nine-component solution, with the three criteria scores for each individual DC task loading onto their own distinct component. This nine-factor structure was confirmed using a confirmatory factor analysis. Results for concurrent validity showed that there was a high positive correlation between DC total score and TGMD-2 and SSA overall score $(r(43)=.86, \mathrm{p}<0.001)$. DC total score showed good test-retest reliability $(\mathrm{ICC}=0.80 ; 95 \% \mathrm{CI}: 0.63,0.90 ; \mathrm{p}$ $<0.001$ ). Inter- and intra-rater reliability on all comparison levels was good (all ICCs $>.85)$.

Overall, this study showed that the DC is a valid and reliable tool to measure elements of physical competence in children aged 10-14 years. 


\section{Thesis Map}

\begin{tabular}{|c|c|c|c|}
\hline 1 & $\begin{array}{l}\text { Results from the Active Healthy Kids } \\
\text { Wales } 2016 \text { Report Card on Physical } \\
\text { Activity for Children and Youth }\end{array}$ & Key Findings & $\begin{array}{l}\text { To consolidate and translate research related to } \\
\text { physical activity among children and youth in } \\
\text { Wales and to synthesise national surveillance } \\
\text { data from several surveys in order to assign } \\
\text { grades to physical activity/inactivity behaviours } \\
\text { and influences indicators, as well as to examine } \\
\text { trends or inequalities in the data. } \\
\text { Grades were assigned as follows; overall } \\
\text { physical activity levels, D+; organized sport } \\
\text { participation, C; active and outdoor play, C; } \\
\text { active transportation, C; sedentary behaviours, } \\
\text { D-; physical literacy, INC; family and peer } \\
\text { influences, D+; school, B; community and the } \\
\text { built environment, C; and national government } \\
\text { policy, strategies and investments, B-. }\end{array}$ \\
\hline \multirow[t]{2}{*}{2} & $\begin{array}{l}\text { Ten-year Secular Changes in } \\
\text { Selected Health and Fitness } \\
\text { Parameters of 10-11 Years Old } \\
\text { Swansea School Children - 2003- } \\
2013\end{array}$ & Aim & $\begin{array}{l}\text { To highlight the secular changes in selected } \\
\text { health and fitness measures (body mass index, } \\
\text { prevalence of overweight and obesity, and grip } \\
\text { strength) in 10-11 year old Swansea school } \\
\text { children, using data obtained in } 2003 \text { and } 2013 .\end{array}$ \\
\hline & & Key Findings & $\begin{array}{l}\text { BMI z-scores, as well as prevalence of } \\
\text { overweight and obesity, decreased significantly } \\
\text { between } 2003 \text { and } 2013 \text { for both boys and girls. } \\
\text { After BMI z-scores adjustment, grip strength } \\
\text { decreased significantly for boys, but not for } \\
\text { girls. }\end{array}$ \\
\hline \multirow[t]{3}{*}{3} & $\begin{array}{l}\text { A Cross-Sectional Study on the } \\
\text { Socio-economic and Sex Differences } \\
\text { in Health-Related Fitness Measures } \\
\text { in School Children }\end{array}$ & $\operatorname{Aim}$ & $\begin{array}{l}\text { To investigate socio-economic and sex } \\
\text { differences in selected health-related fitness } \\
\text { measures (body mass index, MSRT, grip } \\
\text { strength) in 9-12-year-old children. }\end{array}$ \\
\hline & & Key Findings & $\begin{array}{l}\text { Regardless of sex, children in the middle } \\
\text { quintile had a significantly higher mean BMI z- } \\
\text { score than their least deprived counterparts. }\end{array}$ \\
\hline & & & $\begin{array}{l}\text { There was a significant increase in grip } \\
\text { strength and } 20 \mathrm{~m} \text { MSRT between most and } \\
\text { least deprived quintiles. Significant differences } \\
\text { in } 20 \mathrm{~m} \text { MSRT score were more apparent with } \\
\text { increases in socio-economic status in boys. }\end{array}$ \\
\hline \multirow[t]{2}{*}{4} & $\begin{array}{l}\text { A Dynamic Assessment of Children's } \\
\text { Physical Competence: The Dragon } \\
\text { Challenge }\end{array}$ & Aim & $\begin{array}{l}\text { 1. To develop a dynamic measure of physical } \\
\text { competence that requires a participant to } \\
\text { demonstrate fundamental, combined and } \\
\text { complex movement skills, and assessors to } \\
\text { score both processes and products (Dragon } \\
\text { Challenge). } \\
\text { 2. To assess the psychometric properties of the } \\
\text { Dragon Challenge in 10-14 year old children. }\end{array}$ \\
\hline & & $\begin{array}{l}\text { Key } \\
\text { Findings }\end{array}$ & $\begin{array}{l}\text { The DC is a valid and } \\
\text { reliable tool to measure } \\
\text { elements of physical } \\
\text { competence in children aged } \\
10-14 \text { years. }\end{array}$ \\
\hline
\end{tabular}




\begin{tabular}{|c|c|c|c|}
\hline 5 & $\begin{array}{l}\text { Youth Physical } \\
\text { Competence } \\
\text { Promotion Model: A } \\
\text { Quantitative } \\
\text { Investigation into } \\
\text { Modifiable Factors }\end{array}$ & Aim & $\begin{array}{l}\text { To quantify the direct and } \\
\text { indirect relationships } \\
\text { between enabling, } \\
\text { predisposing and reinforcing } \\
\text { factors on physical } \\
\text { competence and to } \\
\text { investigate potential sex, } \\
\text { weight status, and school } \\
\text { level differences. }\end{array}$ \\
\hline & & Key Findings & \\
\hline
\end{tabular}




\section{Study 5}

\subsection{Youth Physical Competence Promotion Model: A Quantita- tive Investigation into Modifiable Factors}

* This chapter is under review for publication:

Tyler, R., Mackintosh, K. A., Foweather, L., Edwards, L. \& Stratton, G. (2018). Youth Physical Competence Promotion Model: A Quantitative Investigation into Modifiable Factors. Scandinavian Journal of Medicine \& Science in Sports (under review).

\subsection{Introduction}

Physical competence, defined as "the sufficiency in movement vocabulary, movement capacities and developed movement patterns plus the deployment of these in a range of movement forms" (p204, Whitehead, 2010), plays an important role in the growth and development of children (Clark \& Metcalfe, 2002; Gallahue et al., 2012; Stodden et al., 2008; Whitehead, 2010). Movement capacities, or movement skills, are imperative to develop, and indeed enhance, physical competence (Whitehead, 2010). Moreover, movement skills consist of three interrelated constructs: fundamental movement skills (FMS; balance, core stability, coordination, speed variation, flexibility, control, proprioception, and power), combined movement (poise, fluency, precision, dexterity, and equilibrium), and complex movement (bilateral coordination, inter-limb coordination, hand-eye coordination, turning, twisting and rhythmic movements, and control of acceleration/deceleration) (Clark \& Metcalfe, 2002; Gallahue et al., 2012; Whitehead, 2010). Whilst FMS develops rapidly from the age of 3 years, children have the potential for FMS mastery by 7-8 years (Clark \& Metcalfe, 2002; Gallahue et al., 2012). Movement patterns, described as general (e.g., sending, striking, receiving, running, jumping, rotating), refined (e.g., throwing, dribbling, catching, sprinting, hopping, turning) and specific (i.e. sport-specific movement patterns), are amalgamations of movements that stem from the selection and application of movement skills (Clark \& Metcalfe, 2002; Gallahue et al., 2012; Whitehead, 2010). More refined and specific movement patterns are achieved when FMS, combined movement skills and complex movement skills are utilised simultaneously (Clark \& Metcalfe, 2002; Gallahue et al., 2012; Whitehead, 2010). Therefore, the development of combined and complex movement skills is speculated to be imperative to increasing levels of physical competence in children over 8 years old (Clark \& Metcalfe, 2002; Gallahue et al., 2012; Tyler et al., 2018; Whitehead, 2010). 
There is a vast array of evidence identifying physical competence as an important component of physical literacy, and, as such, a critical precursor for increasing positive health trajectories, particularly physical activity, across the lifespan (Barnett, Lai, et al., 2016; Edwards et al., 2016; Hulteen et al., 2018; Robinson et al., 2015; Stodden et al., 2008; Whitehead, 2010). Specifically, systematic reviews and longitudinal studies have reported strong evidence for positive associations between physical competence and physical activity levels (Barnett et al., 2009; Holfelder \& Schott, 2014; Lopes et al., 2011; Lubans et al., 2010), health-related fitness (Barnett, van Beurden, et al., 2008; Cattuzzo et al., 2016; Robinson et al., 2015) and perceived competence (Lubans et al., 2010; Robinson et al., 2015), as well as an inverse association with weight status (D'Hondt et al., 2014; Lubans et al., 2010; Robinson et al., 2015), in paediatric populations. Furthermore, studies have shown that enhanced physical competence during childhood tracks across the lifespan by leading to higher levels of physical activity and health-related fitness during adolescence (Barnett et al., 2009; Barnett, van Beurden, et al., 2008; Lopes et al., 2011; Robinson et al., 2015), and by supporting functional independence, general health and quality of life in later life, as well as reducing the risk of all-cause mortality (Hulteen et al., 2018; Paterson \& Warburton, 2010; Sigmundsson et al., 2016). Thus, enhanced physical competence in children and young people is foundational for physical activity promotion and associated health benefits, with transferable value throughout the life course.

Ecological models provide a framework of potential influencing factors on healthrelated behaviours and are useful in emphasising social and psychological influences and environmental contexts (Barnett, Lai, et al., 2016). The Youth Physical Activity Promotion Model (YPAP-M) (Welk, 1999) offers an ecological conceptual model framing factors that may enable (e.g., movement skills/physical competence, healthrelated fitness, environmental attributes, and access), predispose (e.g., perceived competence and self-efficacy) or reinforce (e.g., parental physical activity and family, peer and coach influence) physical activity in children. Although research has investigated the mediating variable framework of the YPAP-M (Chen et al., 2014), the examination of the influencing factors on physical competence, guided by the model, remains to be explored. Further, few studies have investigated both psychological influences and environmental factors on physical competence (Barnett, Lai, et al., 2016). Therefore, the development of an ecological model with physical competence as the endogenous variable would afford new insight and an in-depth understanding of the multiple influences on physical competence. Although the association between phys- 
ical competence and other factors such as physical activity, health-related fitness, and perceived competence, are expected to be reciprocal (Robinson et al., 2015; Stodden et al., 2008), such a model would enable the investigation of factors that could be specifically modified to increase physical competence. Such a targeted approach could therefore inform intervention development with the objective to promote physical competence in children, as well as explain effects or lack of effects in current intervention strategies.

The aim of the current cross-sectional study was to quantify the direct and indirect relationships between enabling, predisposing and reinforcing ecological factors on physical competence and to investigate potential sex, weight status, and school level differences in the model.

\subsection{Participants and Methods}

Following written informed head teacher and parent consent and participant assent, 429 children ( $52 \%$ boys; aged $11.1 \pm 0.6$ years; 87\% white British) from 11 sociodemographically representative primary and secondary schools (Welsh index of multiple deprivation score 23-1898) (Noble et al., 2006) in South Wales, UK, participated in the study between 2015-2018. As part of the serial Swan-Linx programme (Sheldrick et al., 2018; Taylor et al., 2004; Tyler, Mackintosh, Palmer, et al., 2015), participants completed a selection of anthropometric and health-related fitness measures, three questionnaires, and the Dragon Challenge, a previously validated measure of physical competence in 10-14 year old children (Tyler et al., 2018). Ethical approval was obtained from the Institutional Research Ethics Committee [PG/2014/007; PG/2014/037; PG/2016/003].

\subsubsection{Anthropometrics and Health-Related Fitness Measures}

Using standard anthropometric techniques (Lohman et al., 1988), stature and body mass were measured to the nearest $0.001 \mathrm{~m}$ and $0.1 \mathrm{~kg}$, using a portable stadiometer [Seca 213, Seca Ltd, Birmingham, UK] and electronic weighing scales [Seca 876, Seca Ltd, Birmingham, UK], respectively. Body Mass Index (BMI) was calculated and age- and sex-specific BMI cut-points were used to classify overweight and obese participants (Cole et al., 2000). Participants completed two functional health-related fitness tests from the EUROFIT Test Battery (Council of Europe, 1993), the 20m Multistage Shuttle Run Test (20m MSRT), as measure of cardiorespiratory fitness, and the Handgrip Strength Test, as a measure of muscular strength. 


\subsubsection{Questionnaires}

Children completed a 29-item health and lifestyle online questionnaire (Child Health and Activity Tool; CHAT) (see Appendix III), akin to the paper-based SportsLinx Lifestyle Survey (Taylor et al., 2004). Children reported the number of days they had engaged in moderate-to-vigorous physical activity (MVPA), described as "any activity or sport where your heart beats faster, you breathed faster and you felt warmer", for $\geq 60 \mathrm{~min} \cdot \mathrm{day}^{-1}$ in the last week. Children also detailed the number of organised sports clubs they participated in outside of school. Surrounding physical activity facilities were reported by children as the number of areas close to their home that they could play or take part in physical activity in, such as a garden, grassy area/playing field, playground, park, street, leisure/sport centre or school. Children further reported the number of times a week their parent/s or guardian/s engaged in physical activity ( 0 days $=0,1-2$ days $=1,3-4$ days $=2,5$ or more days $=3$ ). Children chose to report both parents/guardians or a single parent/guardian that they live with. Where participants reported two parents/guardians, the parent/guardian with the highest number of physically active times per week was retained. Additionally, participants reported the persons they most prominently participated in physical activity and sport with during and outside of school time (i.e., on their own $(=0)$ or with parents/guardians, siblings, friends, coaches/teachers/other $(=1))$.

Benefits (desired outcomes from taking part) and barriers (perceived blocks or hindrances to taking part) to exercise were measured using a nine-item benefits and tenitem barriers subscale from the Children's Perceived Benefits/Barriers to Exercise Questionnaire (Garcia et al., 1995) (see Appendix VII), using a five-point Likert-type scale, with responses ranging from 1 (disagree a lot) to 5 (agree a lot). A benefits/barriers differential score was calculated by subtracting the mean barriers' score from mean benefits' score, with higher scores indicating greater perceived benefits compared to perceived barriers to exercise.

Perceived adequacy, the perception of capability to achieve some acceptable standard of success, and perceived predilection, the likelihood that one would select a physical activity when given the choice, were measured using a seven-item adequacy and nineitem predilection subscale from the Children's Self-perceptions of Adequacy in and Predilection for Physical Activity Questionnaire (Hay, 1992) (see Appendix VIII). Each item consisted of two mutually exclusive descriptions and, akin to a four-point Likert scale, children decided which of the two descriptions were most like them and whether the selected description was "sort of" or "really" true for them. The 
most inactive or inadequate response was scored as one and the most active/adequate response a four (Hay, 1992). A cumulative score for both adequacy and predilection was calculated from the responses.

\subsubsection{Dragon Challenge}

Details of the Dragon Challenge have been reported elsewhere (Tyler et al., 2018). Briefly, the Dragon Challenge consists of nine tasks (Balance Bench, Core Agility, Wobble Spot, Overarm Throw, Basketball Dribble, Catch, Jumping Patterns, T-Agility, and Sprint) which require the application of a different combination of fundamental, combined and complex movement skills, to form refined and specific movement patterns (Tyler et al., 2018). The Dragon Challenge was administered and assessed using the established methodology (Tyler et al., 2018). A cumulative score (=0-4) for each task was calculated by summing the technique scores and twice the outcome score, with four showing high physical competence at that task (Tyler et al., 2018).

\subsubsection{Statistical Analyses}

Descriptive statistics are presented as mean $\pm \mathrm{SD}$. All statistical tests were completed using SPSS and SPSS AMOS, version 25 [IBM SPSS Statistics Inc., Chicago, IL, USA], with statistical significance set at $\mathrm{p}<0.05$. Missing data $(\mathrm{n}=684,6.9 \%)$ were imputed using an expectation-maximisation algorithm (Nelwamondo, Mohamed, \& Marwala, 2007). A Confirmatory Factor Analysis (CFA) was performed to assess the fit of the measured variables into four hypothesised latent factors (i.e., enabling, reinforcing, predisposing and physical competence). Specifically, the 20m MSRT and the handgrip strength test, as well as responses to questions from the CHAT on MVPA, sport participation and available surrounding physical activity facilities were included as indicators of the enabling factor; the benefits/barriers differential score, the adequacy score, and the predilection score were included as indicators of the predisposing factor; responses to questions on parental/guardian physical activity levels and persons whom participate in physical activity and sport with the participant were included as indicators of the reinforcing factor; and cumulative scores for each Dragon Challenge task were included as indicators of the physical competence factor (Tyler et al., 2018). By default, the factor loading of the first indicator of a latent factor was fixed to 1 . Error terms from the indicator variables were allowed to co-vary within the same factor. Comparative fit index (CFI), Goodness of fit index (GFI), Incremental fit index (IFI), Root mean square error of approximation (RMSEA) and Standardized root mean square residual (SRMR) were used to assess model fit, with CFI, GFI, and IFI of $>0.90$, RMSEA of $<0.05$ and SRMR of $<0.09$ indicating a good fit (Hu \& 
Bentler, 1999; Wang, Fan, \& Wilson, 1996). SEM was then used to evaluate the relationships between enabling, reinforcing, and predisposing latent variables on the physical competence latent variable. The fit was tested at a global level using CFI, GFI, IFI, and RMSEA. Direct effects were measured using direct path coefficients between latent variables. In the case of a mediating latent factor, the indirect effect was measured by taking the product of the two direct effects between the three latent factors. Multi-group comparisons were made using Chi-squared difference tests to determine whether path relationships differed based on the value of a moderator: sex (boys vs. girls), weight status (healthy vs. overweight/obese), and school level (primary vs. secondary). Changes in the CFIs $(\Delta \mathrm{CFI})$ were also calculated for multigroup comparisons, with a value of $\Delta C F I$ less than or equal to -0.01 indicating measurement invariance (Cheung \& Rensvold, 2002). Paths that were non-significant at an overall level, as well as for all values of the moderators, were removed from the final SEM.

\subsection{Results}

Mean and standard deviations of the measured variables are presented in Table 16. 
Table 16. Descriptive statistics (mean $\pm S D$ ) of Study 5 measured variables

\begin{tabular}{|c|c|c|c|}
\hline Variables & Boys & Girls & All \\
\hline MVPA (0-7 days) & $2.4 \pm 1.2$ & $2.2 \pm 1.1$ & $2.3 \pm 1.2$ \\
\hline Sport Participation (number of sports) & $2.7 \pm 2.7$ & $2.3 \pm 2.1$ & $2.5 \pm 2.4$ \\
\hline Surrounding PA Facilities ( $0-8$ facilities) & $3.0 \pm 2.0$ & $3.2 \pm 2.0$ & $3.1 \pm 2.0$ \\
\hline Grip Strength (kg) & $17.7 \pm 3.9$ & $17.1 \pm 3.8$ & $17.4 \pm 3.8$ \\
\hline 20m MSRT (shuttles) & $31.9 \pm 18.1$ & $22.5 \pm 11.9$ & $27.4 \pm 16.1$ \\
\hline Out of School PA/Sport with (0-1) & $0.9 \pm 0.3$ & $0.8 \pm 0.4$ & $0.9 \pm 0.3$ \\
\hline School PA/Sport with (0-1) & $0.9 \pm 0.2$ & $0.9 \pm 0.2$ & $0.9 \pm 0.2$ \\
\hline Parents PA Levels (0-3) & $1.5 \pm 0.9$ & $1.7 \pm 0.9$ & $1.6 \pm 0.9$ \\
\hline Predilection (9-36) & $28.3 \pm 4.5$ & $28.2 \pm 5.3$ & $28.2 \pm 4.9$ \\
\hline Benefits/Barriers to PA (-41-35) & $1.4 \pm 0.9$ & $1.3 \pm 0.8$ & $1.4 \pm 0.8$ \\
\hline Adequacy (7-28) & $21.1 \pm 3.6$ & $20.8 \pm 3.7$ & $20.9 \pm 3.6$ \\
\hline Balance Bench (0-4) & $1.4 \pm 1.1$ & $1.6 \pm 1.2$ & $1.5 \pm 1.1$ \\
\hline Core Agility (0-4) & $1.3 \pm 0.9$ & $1.5 \pm 1.0$ & $1.4 \pm 1.0$ \\
\hline Wobble Spot (0-4) & $1.3 \pm 1.5$ & $1.4 \pm 1.5$ & $1.4 \pm 1.5$ \\
\hline Overarm Throw (0-4) & $2.1 \pm 0.9$ & $1.3 \pm 1.0$ & $1.7 \pm 1.0$ \\
\hline Basketball Dribble (0-4) & $2.3 \pm 1.0$ & $1.7 \pm 1.2$ & $2.0 \pm 1.1$ \\
\hline Catch $(0-4)$ & $1.5 \pm 1.3$ & $0.9 \pm 1.1$ & $1.2 \pm 1.3$ \\
\hline T-Agility (0-4) & $1.3 \pm 1.1$ & $1.2 \pm 1.0$ & $1.2 \pm 1.1$ \\
\hline Jumping Patterns (0-4) & $2.0 \pm 1.0$ & $2.0 \pm 1.0$ & $2.0 \pm 1.0$ \\
\hline Sprint $(0-4)$ & $2.5 \pm 0.8$ & $2.3 \pm 0.9$ & $2.4 \pm 0.8$ \\
\hline
\end{tabular}

Note. MVPA = Moderate-to-vigorous physical activity; PA = Physical activity; 20m MSRT $=20 \mathrm{~m}$ Multistage Shuttle Run Test

Confirmatory Factor Analysis: The fit for the hypothesised CFA (Figure 6) was good (CFI, 0.927; GFI, 0.945; IFI, 0.928; RMSEA, 0.035 90\% CI 0.026 - 0.044; SRMR, 0.050), after the addition of three correlations between error terms within the same factor. 


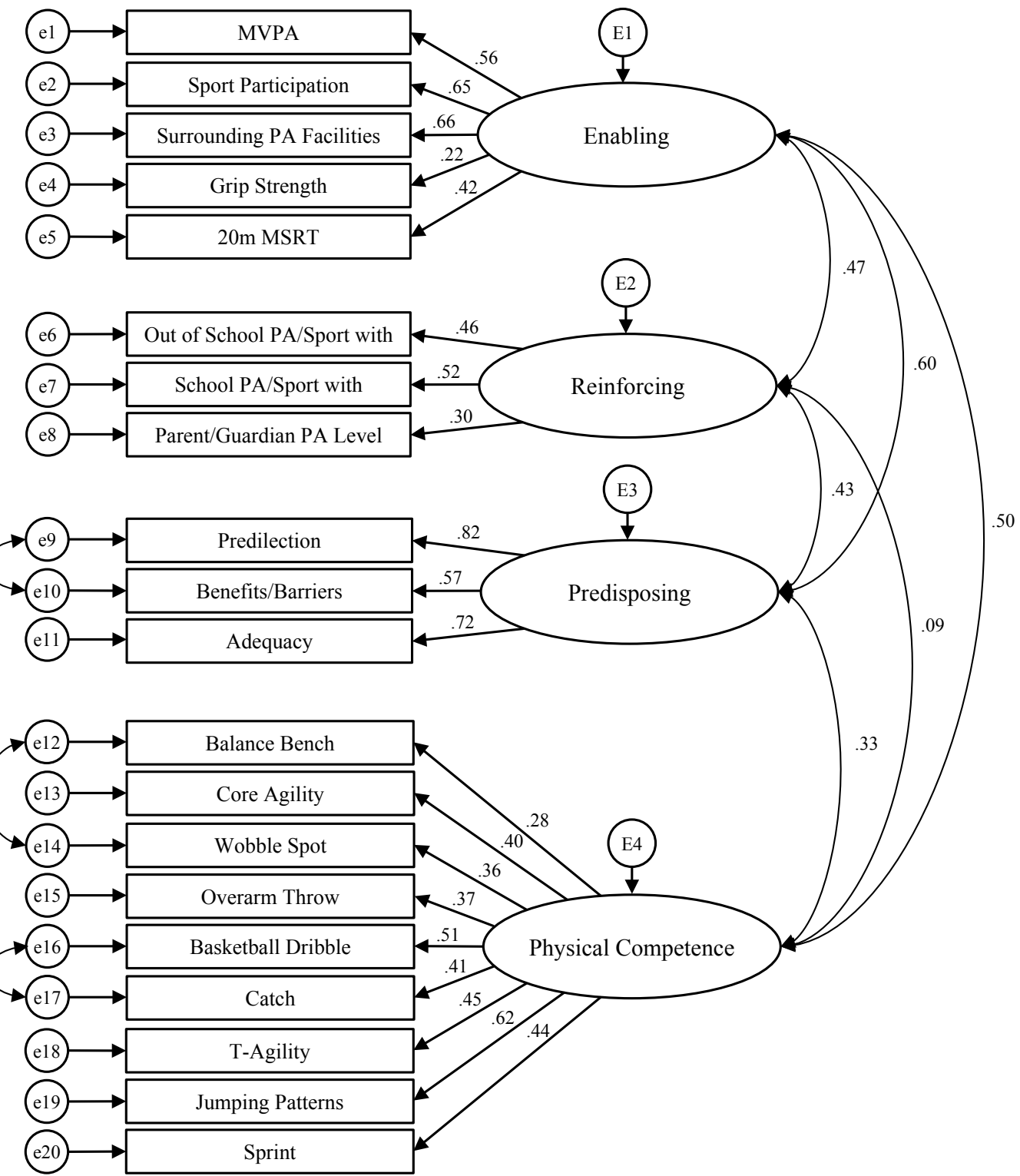

Figure 6. Confirmatory Factor Analysis of the measured variables into four hypothesised latent factors

Structural Equation Model: The hypothesised SEM is shown in Figure 7. The paths from (i) the reinforcing factor to the physical competence factor and (ii) the predisposing factor to the physical competence factor were not significant $(\mathrm{p}>0.05)$. Moreover, these relationships did not differ significantly based on the value of any of the moderators (i.e., boys vs. girls, healthy vs. overweight/obese, primary vs. secondary school level), and so both paths were removed in the final model. Post-hoc power analysis identified sufficiency to detect significant effects (statistical power $>0.8$ ). 


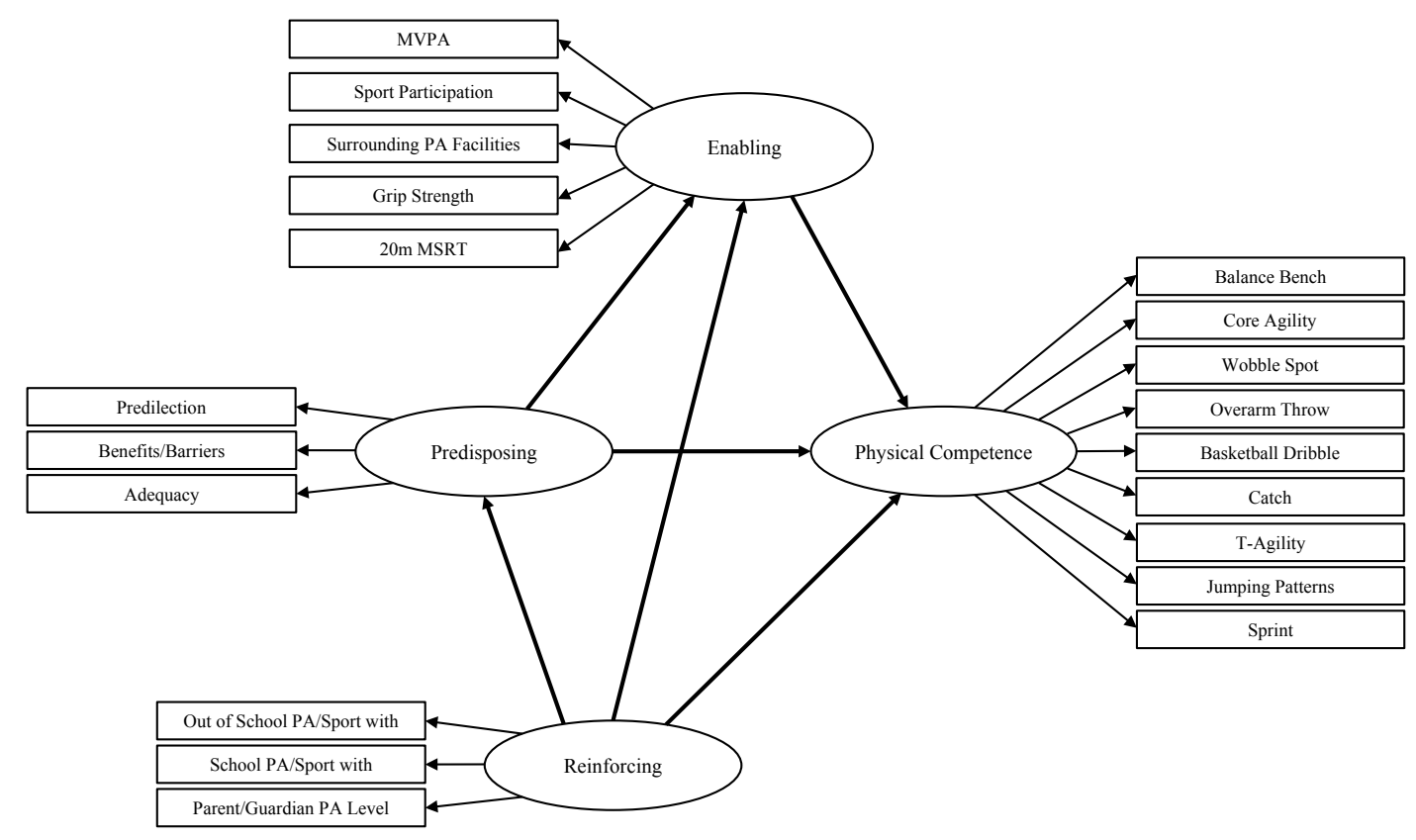

Figure 7. Hypothesised Structural Equation Model

The final SEM (Figure 8) demonstrated a good fit on a global level (CFI, 0.925; GFI, 0.944; IFI, 0.927; RMSEA, 0.035 90\% CI 0.026 - 0.044; SRMR, 0.050). The model revealed that the reinforcing factor was directly related to the predisposing ( $\beta=0.42$, $\mathrm{p}<0.001)$ and enabling factors $(\beta=0.22, \mathrm{p}=0.031)$. An indirect relationship was found between the reinforcing and physical competence factors, mediated by the enabling factor $(\beta=0.11, \mathrm{p}=0.025)$. The predisposing factor was found to have a direct effect on the enabling factor $(\beta=0.51, \mathrm{p}<0.001)$, and an indirect effect on physical competence mediated by the enabling factor $(\beta=0.26, \mathrm{p}<0.001)$. The enabling factor had a direct effect on the physical competence factor $(\beta=0.50, \mathrm{p}<0.001)$. Multi-group comparisons showed that each of these direct effects did not differ $(\mathrm{p}$ $>0.05$ ) by sex $(\Delta \mathrm{CFI},-0.021)$, weight status $(\Delta \mathrm{CFI},-0.014)$, or school level $(\Delta \mathrm{CFI}$, $-0.017)$. 


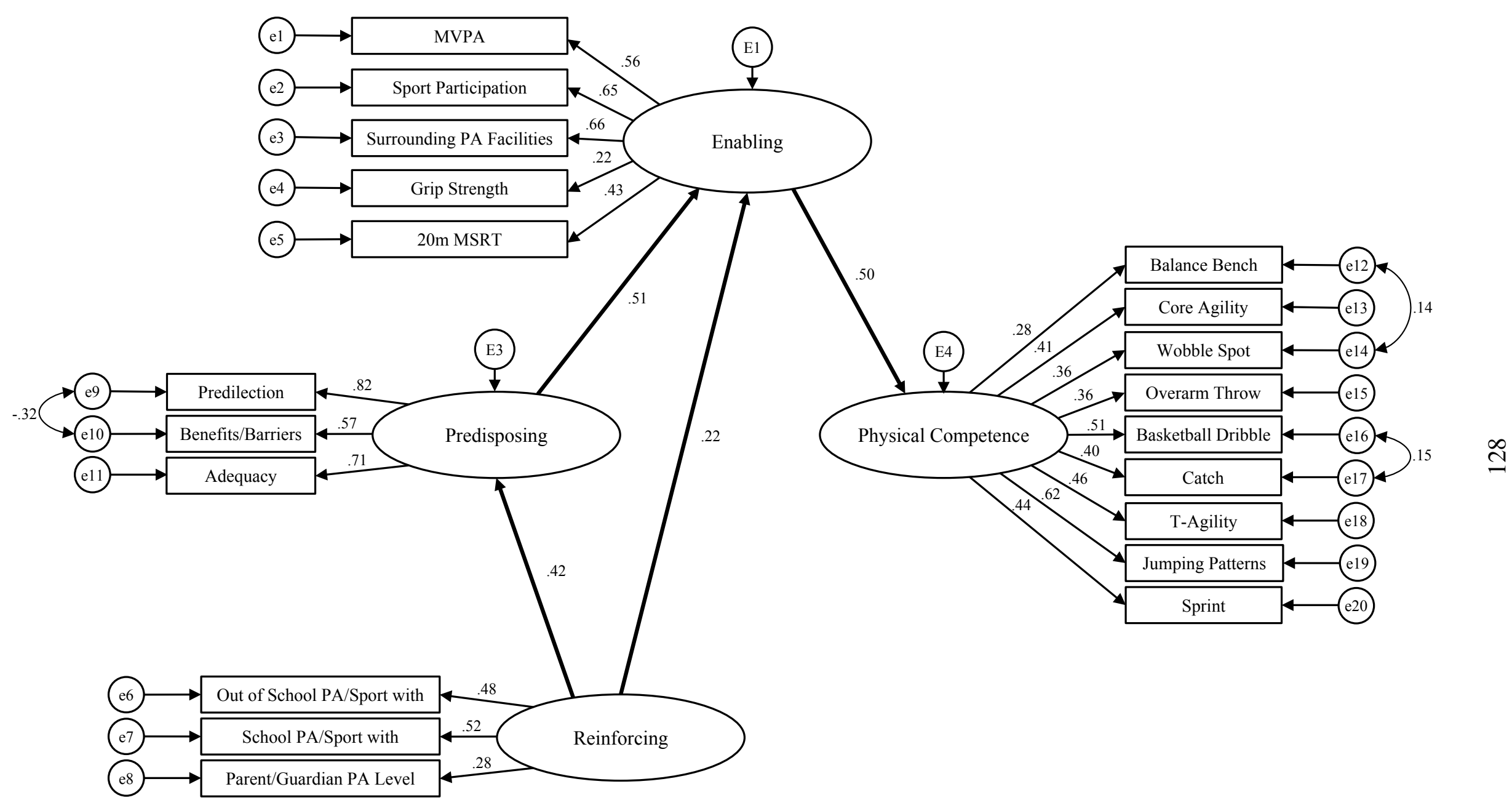

Figure 8. Final Structural Equation Model evaluating the relationships between enabling, reinforcing, predisposing and physical competence latent variables 


\subsection{Discussion}

This is the first study to report the direct and indirect relationships between enabling, predisposing, and reinforcing factors on physical competence. This study presents an ecological model with physical competence as the endogenous variable to provide understanding of the multiple influences on such an outcome (Barnett, Lai, et al., 2016). Results from the CFA showed that the fit of the measured variables into the four hypothesised latent factors based on the YPAP-M (Welk, 1999) was good, confirming that the selected measures were associated with the appropriate latent factor.

The finding that the enabling factor had a direct effect on the physical competence factor purports that an increase in the enabling factor resulted in an increase in physical competence, and thus an improvement in competence in movement skills and advanced movement patterns. In accord with systematic reviews, there was a positive association between physical competence and MVPA (Barnett, Lai, et al., 2016; Barnett et al., 2009; Holfelder \& Schott, 2014; Lopes et al., 2011; Lubans et al., 2010; Robinson et al., 2015), sport participation (Barnett, Lai, et al., 2016; Henrique et al., 2016; Holfelder \& Schott, 2014; Robinson et al., 2015) and aspects of health-related fitness (Barnett, van Beurden, et al., 2008; Cattuzzo et al., 2016; Robinson et al., 2015; Stodden, Gao, Goodway, \& Langendorfer, 2014). Further, research suggests that a positive feedback loop exists, in which children with greater levels of physical activity and sport participation develop better physical competence and fitness, consequently further increasing engagement (Robinson et al., 2015; Stodden et al., 2008). Whilst environmental and access factors have been previously reported to support physical activity (Bauman et al., 2012; Welk, 1999), little evidence has shown the impact on physical competence (Barnett, Lai, et al., 2016). It is therefore noteworthy that available surrounding physical activity facilities loaded onto the enabling factor, which was positively associated with physical competence. Overall, the finding that the enabling factor had a direct effect on physical competence supports previous literature (Barnett, Lai, et al., 2016; Barnett et al., 2009; Barnett, van Beurden, et al., 2008; Cattuzzo et al., 2016; Henrique et al., 2016; Holfelder \& Schott, 2014; Lopes et al., 2011; Lubans et al., 2010; Robinson et al., 2015; Stodden et al., 2014), as well as provides further evidence of an association between physical activity, sport participation, fitness, and surrounding facilities and physical competence.

In line with previous research that has displayed positive associations between parental influence and family support (reinforcing variables), and physical activity levels and fitness (enabling variables), in children and adolescents (Bauman et al., 2012; Welk, 
1999), the direct relationship between the reinforcing and enabling factor further supports the importance of parental/guardian modelling and friends and family encouragement. Conversely, few studies have demonstrated that reinforcing variables can simultaneously influence predisposing variables (Chen et al., 2014). Therefore, the proposed model is of importance since it shows that an increase in the reinforcing factor resulted in an increase in perceived benefits to, adequacy in, and predilection to physical activity (predisposing factor). Given that previous literature has shown a parental influence on movement skills competence (Cools, De Martelaer, Samaey, \& Andries, 2011), it was hypothesised that the reinforcing factor would also have a direct relationship on the physical competence factor, though this direct relationship was not apparent in the current findings. Rather, results showed an indirect relationship between the reinforcing factor and physical competence factor, mediated by the enabling factor. Consequently, increasing the reinforcing factor (i.e., social support and modelling) may result in improvements in enabling measured variables as well as physical competence. Overall, the findings in the current study regarding the reinforcing factor provide evidence for the impact of psychosocial variables on biological, environmental, behavioural, and psychological variables, as well as indirectly on physical competence levels.

Congruent with previous research, whereby higher levels of self-efficacy, perceived competence, and overall physical competence were related to higher levels of physical activity (Babic et al., 2014; Bauman et al., 2012), the SEM showed that the predisposing factor had a direct effect on the enabling factor. While it was hypothesised that the predisposing factor may have a direct relationship on the physical competence factor, an indirect effect, mediated by the enabling factor, was found. Indeed, previous research has shown that perceived competence has a mediating effect on the association between physical competence and physical activity in children and adolescents (Barnett, Morgan, van Beurden, Ball, \& Lubans, 2011; Barnett, Morgan, van Beurden, $\&$ Beard, 2008). This study therefore provides further support to the contention that an increase in the predisposing factor will result in an increase in physical activity, sport participation, and health-related fitness, and subsequently an increase in levels of physical competence.

Overall, previous research supports the synergistic relationships of biological, environmental, psychosocial and behavioural factors on the evolution and continued development of physical competence across the lifespan (Barnett, Lai, et al., 2016; Hulteen et al., 2018; Robinson et al., 2015; Stodden et al., 2008). The current study 
further supports the strength of these relationships, particularly in terms of promoting physical competence in an ecological model that can be used to inform interventions. One such intervention strategy would be to promote physical activity, sport participation, health-related fitness, and available surrounding physical activity facilities, given the direct effect of the enabling factor on physical competence in the current results. Moreover, whilst both the reinforcing and predisposing factor only had a direct effect on the enabling factor, the indirect effect of these factors on physical competence, indicates that an increase in either reinforcing or predisposing factor was indirectly associated with an increase in physical competence. Thus, interventions to promote physical competence could also focus on enhancing social support mechanisms such as parental/guardian physical activity levels, the number of persons whom take part in physical activity and sport with children, and children's perceived benefits to, adequacy in, and predilection to physical activity, while decreasing children's perceived barriers to physical activity. Contrary to previous findings that show increasing age, healthy weight status and being male are correlates for certain aspects of physical competence (Barnett, Lai, et al., 2016), multi-group comparisons did not display these differences. Consequently, the SEM revealed an ecological model that can be used to inform interventions for the improvement of physical competence in children via multiple pathways regardless of age, weight status, and sex.

\subsubsection{Strengths and Limitations}

The use of SEM in the current study provides a novel approach to identifying modifiable factors that can increase physical competence in children, allowing the investigation of the concurrent influences of multiple variables. Indeed, SEM explicitly models measurement error, thereby providing more accurate relationships among latent factors, a frequently cited limitation of many studies (Beran \& Violato, 2010). Furthermore, the assessment of fundamental, combined and complex movement skills and varying complexities of movement patterns provides a more inclusive measure to inform physical competence (Tyler et al., 2018; Whitehead, 2010).

Whilst there are numerous strengths, the current study is not without limitations. Specifically, the measures chosen to best predict each latent variable in the model were selected from the measures involved in the Swan-Linx programme, and therefore other quantitative measures (e.g., accelerometer data rather than self-report physical activity) may have increased the strength of the model. Future research could also expand the measures used to assess enabling and reinforcing factors (e.g., reinforcing factors could include encouragement for physical competence from peers and parents 
or other aspects of social support). Further, an expectation-maximisation algorithm was used to impute missing data and, although this imputation method has previously been validated (Nelwamondo et al., 2007), the lack of a complete data set may have influenced study findings. Finally, although no differences were found between primary and secondary school level children, it is possible that age differences may be apparent with a larger age-range, or that biological age may account for greater variation.

\subsection{Conclusion}

In conclusion, the present study found that the enabling factor had a direct effect, whilst the reinforcing and predisposing factors had an indirect effect, on physical competence. Each direct and indirect effect did not differ by school level (primary school vs. secondary school), weight status (healthy vs. overweight and obese) or sex (boys vs. girls), supporting the contention that the model is applicable across many groups of primary and secondary level school children. These findings are the first to be set in this framework and reveal that there are multiple potential pathways that could inform future interventions that aim to promote physical competence.

\subsection{Summary: Study 5}

The aims of this study were to quantify the relationships between enabling, predisposing and reinforcing ecological factors on physical competence and investigate potential sex, weight status, and school level differences.

Cardiorespiratory fitness (20m Multistage Shuttle Run), muscular strength (Handgrip Strength) and online questionnaire (Child Health and Activity Tool; CHAT) data on moderate-to-vigorous physical activity, sport participation and available surrounding physical activity facilities were included as enabling variables. Three predisposing variables were determined from self-report data on benefits/barriers to exercise, adequacy, and predilection. Parental/guardian physical activity levels and persons whom participate in physical activity and sport with the participant (CHAT) were selected as reinforcing variables. Physical competence was determined from cumulative scores for Dragon Challenge tasks.

Physical competence was directly affected by the enabling factor $(\beta=0.50, \mathrm{p}<0.001)$ but indirectly affected by reinforcing and predisposing factors, mediated by the enabling factor $(\beta=0.11, \mathrm{p}=0.025 ; \beta=0.26, \mathrm{p}<0.001)$. Multi-group comparisons showed 
that each of these effects did not differ by sex, weight status or school level ( $p>0.05)$.

Physical competence is an important component of physical literacy in children and this study demonstrated that enabling factors (e.g., fitness, physical activity) are crucial for its development. Reinforcing and predisposing factors are themselves indirectly associated with physical competence, highlighting alternate pathways for improvements. This is the first study to quantify an ecological model with physical competence as the endogenous variable and is key to interventions that aim to promote physical competence. 


\section{Thesis Map}

Study

Outcomes

\begin{tabular}{|c|c|c|c|}
\hline 1 & $\begin{array}{l}\text { Results from the Active } \\
\text { Healthy Kids Wales } \\
\text { 2016 Report Card on } \\
\text { Physical Activity for } \\
\text { Children and Youth }\end{array}$ & $\begin{array}{c}\text { Key } \\
\text { Findings }\end{array}$ & $\begin{array}{l}\text { To consolidate and translate } \\
\text { research related to physical } \\
\text { activity among children and } \\
\text { youth in Wales and to } \\
\text { synthesise national surveillance } \\
\text { data from several surveys in } \\
\text { order to assign grades to } \\
\text { physical activity/inactivity } \\
\text { behaviours and influences } \\
\text { indicators, as well as to examine } \\
\text { trends or inequalities in the data. } \\
\text { Grades were assigned as } \\
\text { follows; overall physical } \\
\text { activity levels, D+; organized } \\
\text { sport participation, C; active and } \\
\text { outdoor play, C; active } \\
\text { transportation, C; sedentary } \\
\text { behaviours, D-; physical } \\
\text { literacy, INC; family and peer } \\
\text { influences, D+; school, B; } \\
\text { community and the built } \\
\text { environment, C; and national } \\
\text { government policy, strategies } \\
\text { and investments, B-. }\end{array}$ \\
\hline 2 & $\begin{array}{l}\text { Ten-year Secular } \\
\text { Changes in Selected } \\
\text { Health and Fitness } \\
\text { Parameters of 10-11 } \\
\text { Years Old Swansea } \\
\text { School Children - 2003- } \\
2013\end{array}$ & $\begin{array}{c}\text { Aim } \\
\\
\\
\\
\text { Key } \\
\text { Findings }\end{array}$ & $\begin{array}{l}\text { To highlight the secular changes } \\
\text { in selected health and fitness } \\
\text { measures (body mass index, } \\
\text { prevalence of overweight and } \\
\text { obesity, and grip strength) in } \\
10-11 \text { year old Swansea school } \\
\text { children, using data obtained in } \\
2003 \text { and } 2013 \text {. } \\
\text { BMI z-scores, as well as } \\
\text { prevalence of overweight and } \\
\text { obesity, decreased significantly } \\
\text { between } 2003 \text { and } 2013 \text { for both } \\
\text { boys and girls. After BMI z- } \\
\text { scores adjustment, grip strength } \\
\text { decreased significantly for boys, } \\
\text { but not for girls. }\end{array}$ \\
\hline
\end{tabular}




\begin{tabular}{|c|c|c|c|}
\hline \multirow[t]{2}{*}{3} & $\begin{array}{l}\text { A Cross-Sectional } \\
\text { Study on the Socio- } \\
\text { economic and Sex } \\
\text { Differences in Health- } \\
\text { Related Fitness } \\
\text { Measures in School } \\
\text { Children }\end{array}$ & Aim & $\begin{array}{l}\text { To investigate socio- } \\
\text { economic and sex differences } \\
\text { in selected health-related } \\
\text { fitness measures (body mass } \\
\text { index, MSRT, grip strength) } \\
\text { in 9-12-year-old children. }\end{array}$ \\
\hline & & $\begin{array}{c}\text { Key } \\
\text { Findings }\end{array}$ & $\begin{array}{l}\text { Regardless of sex, children in } \\
\text { the middle quintile had a } \\
\text { significantly higher mean } \\
\text { BMI z-score than their least } \\
\text { deprived counterparts. There } \\
\text { was a significant increase in } \\
\text { grip strength and } 20 \mathrm{~m} \text { MSRT } \\
\text { between most and least } \\
\text { deprived quintiles. } \\
\text { Significant differences in } \\
20 \mathrm{~m} \text { MSRT score were more } \\
\text { apparent with increases in } \\
\text { socio-economic status in } \\
\text { boys. }\end{array}$ \\
\hline \multirow[t]{2}{*}{4} & $\begin{array}{l}\text { A Dynamic } \\
\text { Assessment of } \\
\text { Children's Physical } \\
\text { Competence: The } \\
\text { Dragon Challenge }\end{array}$ & Aim & $\begin{array}{l}\text { 1. To develop a dynamic } \\
\text { measure of physical } \\
\text { competence that requires a } \\
\text { participant to demonstrate } \\
\text { fundamental, combined and } \\
\text { complex movement skills, } \\
\text { and assessors to score both } \\
\text { processes and products } \\
\text { (Dragon Challenge). } \\
2 . \text { To assess the psychometric } \\
\text { properties of the Dragon } \\
\text { Challenge in 10-14 year old } \\
\text { children. }\end{array}$ \\
\hline & & $\begin{array}{c}\text { Key } \\
\text { Findings }\end{array}$ & $\begin{array}{l}\text { The DC is a valid and } \\
\text { reliable tool to measure } \\
\text { elements of physical } \\
\text { competence in children aged } \\
10-14 \text { years. }\end{array}$ \\
\hline
\end{tabular}




\begin{tabular}{|c|c|c|c|}
\hline 5 & $\begin{array}{l}\text { Youth Physical } \\
\text { Competence } \\
\text { Promotion Model: A } \\
\text { Quantitative } \\
\text { Investigation into } \\
\text { Modifiable Factors }\end{array}$ & Aim & $\begin{array}{l}\text { To quantify the direct and } \\
\text { indirect relationships } \\
\text { between enabling, } \\
\text { predisposing and reinforcing } \\
\text { factors on physical } \\
\text { competence and to } \\
\text { investigate potential sex, } \\
\text { weight status, and school } \\
\text { level differences. }\end{array}$ \\
\hline & & $\begin{array}{c}\text { Key } \\
\text { Findings }\end{array}$ & $\begin{array}{l}\text { The final SEM (Figure 8) } \\
\text { demonstrated a good fit. The } \\
\text { enabling factor had a direct } \\
\text { effect, whilst the reinforcing } \\
\text { and predisposing factors had } \\
\text { an indirect effect, on physical } \\
\text { competence. Each direct and } \\
\text { indirect effect did not differ } \\
\text { by school level, weight status } \\
\text { or sex. Thus, the ecological } \\
\text { model revealed multiple } \\
\text { potential pathways that could } \\
\text { inform future interventions } \\
\text { that aim to promote physical } \\
\text { competence. }\end{array}$ \\
\hline
\end{tabular}




\section{Thesis Synthesis}

The overall aim of the thesis was to investigate the health, fitness, lifestyle and physical competency levels and inequalities of school children in Wales. This thesis is a result of an iterative process, with each study addressing a gap/rationale or future direction brought forward by the previous studies. The novelty of this thesis stands in the five studies conducted within. The monitoring of health-related fitness measures and physical competence, as well as the surveillance of physical activity behaviours and influences affords new findings to the current evidence base on children's present and future health and provides insight for policies and interventions across many sectors (e.g., health, sport and recreation, education, transportation and land-use planning). In addition, the development of a new dynamic land-based measure of physical competence, which is valid and reliable in children, as well as the quantification of an ecological model with physical competence as the endogenous variable, provides novel means of physical competence assessment and promotion.

\subsection{Physical activity behaviours and influences and health-related fitness}

Physical activity is associated with numerous positive physiological, psychosocial and cognitive health benefits in children (Biddle \& Asare, 2011; Boreham \& McKay, 2011; Janssen \& Leblanc, 2010), including the prevention of emerging health risk factors (Andersen et al., 2011; Janssen \& Leblanc, 2010). It has been suggested that physical activity levels and behaviours should be a focus for surveillance, and trends in physical activity across multiple domains should be monitored at the individual, social, environmental, and societal levels for policy and intervention development (Bauman et al., 2012; Hallal et al., 2012; Kohl et al., 2012; Sallis et al., 2016). Furthermore, it has been postulated that assessing products or outcomes of physical activity, such as physical fitness, can provide an indication of the quantity and quality of physical activity (Guinhouya, 2012). Studies 1, 2 and 3 were developed following these recommendations.

Collectively, Studies 1, 2 and 3 demonstrated that despite the existence of theoretically grounded policies, programmes, and infrastructure, only 20 to $39 \%$ of children and young people in Wales met the government recommendation of at least 60 minutes of moderate-to-vigorous intensity physical activity (MVPA) every day of the week. Moreover, sedentary behaviour was high ( $>65 \%$ of children and young people spent $2+$ hours a day in sedentary behaviours) and physical activity behaviours and influ- 
ences, including play, sport participation, active transportation and family and peer influences, low. In addition, sedentary behaviour and physical activity levels were one of the highest and lowest, respectively, globally (Inchley et al., 2016; Tyler et al., 2016). Cardiorespiratory fitness and muscular strength levels in children were low to moderate in comparison to international norms (Tomkinson, Carver, et al., 2017; Tomkinson, Lang, Tremblay, et al., 2017) and the prevalence of overweight and obesity high. Analysis of trends and inequalities in physical activity behaviours and influences and health-related fitness in Studies 1, 2 and 3 found no significant change MVPA from 2006 to 2014, although an upward trend in the frequency of organised sport participation between 2011, 2013 and 2015 was apparent. Further, between 2003 and 2013, body mass index (BMI) z-scores decreased significantly even though the prevalence of overweight and obesity recorded in 2013 remained high. Grip strength also decreased significantly for boys, but not for girls. The decreases in both BMI Z-score and grip strength could, in part, be explained by the decrease in participation in extracurricular sports in children between 2002 and 2013 (Sport Wales, 2015c). In terms of inequalities, boys were more active than girls across all age and socioeconomic status groups. However, there were no significant differences in MVPA by socio-economic status, unlike sport participation. Boys also had a significantly higher grip strength and achieved a significantly greater $20 \mathrm{~m}$ multistage shuttle run test score than girls. Finally, regardless of sex, children in the middle Welsh Index of Multiple Deprivation (WIMD) quintile had a significantly higher mean BMI z-score, and most deprived children had significantly lower muscular strength and cardiorespiratory fitness, than their least deprived counterparts.

Taken together, Studies 1, 2 and 3 showed that physical activity behaviours and influences and overall health-related fitness levels were low, and inequalities existed across socio-economic status and sex in children and young people in Wales. Previous literature has shown that school-based interventions play an important role in promoting and improving physical fitness (Minatto, Barbosa Filho, Berria, \& Petroski, 2016). Therefore, targeted intervention strategies aiming to improve children and young people's physical activity and fitness and greater investments are needed, particularly in areas of high deprivation and in girls, so that future generations receive the health and developmental benefits associated with physical activity and fitness.

\subsection{Physical competence monitoring and promotion}

Other than health-related fitness as an outcome measure of children and young people's physical activity levels, the development of motor skills and physical compe- 
tence should be viewed as an additional and independent psychomotor factor related to physical activity that requires specific analyses (Guinhouya, 2012). Physical competence plays an important role in the growth and development of children (Clark \& Metcalfe, 2002; Gallahue et al., 2012; Stodden et al., 2008; Whitehead, 2010). Further, there is a vast array of evidence identifying physical competence as an important component of physical literacy, and, as such, a critical precursor for increasing positive health trajectories, particularly physical activity and health-related fitness, across the lifespan (Barnett, Lai, et al., 2016; Edwards et al., 2016; Hulteen et al., 2018; Robinson et al., 2015; Stodden et al., 2008; Whitehead, 2010). To develop successful targeted interventions to improve physical competence, capturing the current levels of physical competence in children is required (Study 4). Moreover, the prioritisation of key psychosocial, behavioural, physical and environmental factors is needed to promote physical competence in children (Study 5).

Due to the emphasis placed on physical literacy by Sport Wales and the Education Department of Welsh Government, physical literacy, including physical competence, was an indicator considered in Study 1. However, this indicator received an inconclusive grade based off the on-going development of a measurement tool for physical competence in older age children. Study 4 addressed this gap by developing a dynamic measure of physical competence (Dragon Challenge). Many previous measures that inform physical competency as part of physical literacy assessments (Giblin et al., 2014; Robinson \& Randall, 2017), in children and adolescents (Bruininks \& Bruininks, 2005; Cairney et al., 2018; Henderson et al., 2007; Physical and Health Education Canada (PHE Canada), 2014; Ulrich, 2000), use isolated movement skills, which limits transferability and applicability to multi-skill and sport environments and ignores perception-action coupling. The Dragon Challenge, however, includes 9 tasks completed in a timed circuit, and incorporates fundamental, combined and complex movement skills/capacities in a series of refined/complex and specific movement patterns. The Dragon Challenge can be used for assessment for learning (summative and/or formative) by teachers, coaches and health professionals, and because of its time efficiency as a national surveillance tool, that can be aligned to physical literacy programmes and physical education curriculum. The finalised assessment criteria for the Dragon Challenge included both technique (process) and outcome (product) indicators of movement performance, to provide a more complete picture of physical competence levels than currently used assessments that include primarily product- or process-based criteria (Bruininks \& Bruininks, 2005; Cairney et al., 2018; Henderson et al., 2007; Physical and Health Education Canada (PHE Canada), 2014; Ulrich, 
2000). Favourable results in support of concurrent validity, as well as, good test-retest and inter- and intra-rater reliability were found, showing that the Dragon Challenge has sufficient psychometric properties. Therefore, given the paucity of dynamic measures of movement skills and varying complexities of movement patterns to inform physical competence in children, Study 4 filled a critical gap in the current literature in this field. The Dragon Challenge pilot data and initial results presented in Study 1, as well as the descriptive statistics of Dragon Challenge scores and successful demonstration of each Dragon Challenge criterion displayed in Study 4, suggest that continued investment in developing skilled movements in children is required.

Study 4 also confirmed that each of the nine first order latent factors (skill combinations) loaded onto a higher order latent factor (physical competence), purporting that the combination of skills required by each Dragon Challenge task is needed for children to be physically competent. Therefore, to promote physical competence in children, all types of movement skills should aim to be enhanced. To further develop successful targeted interventions, an appropriate conceptual promotion model should be utilised to prioritise the key characteristics of the target group and to produce a comprehensive and structured assessment of needs and barriers to physical competence (Green et al., 1980; Welk, 1999). The development of the Dragon Challenge provided the opportunity to investigate such a model, with physical competence as the endogenous variable. Study 5 resulted in the first reported model that quantified the direct and indirect relationships between ecological factors on physical competence. Previous research supports the synergistic relationships of biological, environmental, psychosocial and behavioural factors on the evolution and continued development of physical competence across the lifespan (Barnett, Lai, et al., 2016; Hulteen et al., 2018; Robinson et al., 2015; Stodden et al., 2008). The results from Study 5 further supported the strength of these relationships, and the promotion of physical competence in a single model.

Congruent with past studies, whereby higher levels of self-efficacy, perceived competence and overall physical competence were related to higher levels of physical activity (Babic et al., 2014; Bauman et al., 2012), the results of Study 5 suggest that augmenting predisposing variables will result in an increase in physical activity, sport participation, and health-related fitness, and subsequently an increase in levels of physical competence. Moreover, in line with previous research that has found positive associations between parental influence and family support (reinforcing variables), and physical activity levels and fitness (enabling variables), in children and 
adolescents (Bauman et al., 2012; Welk, 1999), the direct relationship between the reinforcing and enabling factor, and the indirect relationship between reinforcing and physical competence highlights the importance of parental/guardian modelling and friends and family encouragement. This finding is also consistent with the results from the first study in this thesis that reported a parental/peer influence on children's participation in sport. Finally, given that Study 5 showed a strong direct effect of the enabling factor on physical competence, and Studies 1, 2 and 3 show that levels of physical activity and health-related fitness in children in Wales are low, it is clear that a concerted effort is needed to increase cardiorespiratory fitness, muscular strength, MVPA, sport participation and access to physical activity facilities. This effort is necessary not just for the decrease in potential health risk factors, but also for healthy growth and development of children.

\subsection{Strengths and limitations}

The major strength of this thesis is the novelty of Studies $2-5$, which build on the position established in Study 1. Studies 2 and 3 were the first to highlight the secular changes in selected health-related fitness measures and the inequalities in selected health-related fitness across socio-economic and sex, in children in Wales, respectively. Study 3 was the first to compare the health-related fitness levels of children in Wales to international normative values. Study 5 was the first to quantify an ecological framework to promote physical competence. Moreover, the novelty of the thesis also stands in the systematic development of a new dynamic land-based measure of physical competence in Study 4. Through the use of an in-depth analysis of a large nationally representative dataset and robust methodology guided by the COSMIN (Consensus-based Standards for the selection of health Measurement Instruments), Study 4 produced a vigorous evaluation of the psychometric properties of the Dragon Challenge that would achieve a quality level of good to excellent on the COSMIN rating system (Mokkink et al., 2010).

Although there are numerous strengths, the empirical work in the thesis is not without limitations. Whilst the process behind Study 1 was built on a robust approach and completed results were obtained for 10 out of 11 indicators on physical activity behaviours and influences, there were significant gaps unveiled in the available data and inconsistency across tools used to generate the data to produce the Active Healthy Kids Wales Report Card. A more complete database may have produced different results. Additionally, the results of the Report Card are based off the overall consensus of an expert group, therefore introducing potential subjectivity within the grades. 
The relatively small sample size (512 participants in 2003; 414 participants in 2013) in Study 2 may have limited statistical power, and thus increased the possibility of making a type II error. Furthermore, the participants in Study 2 were from schools situated in the same electoral wards (areas), and therefore the findings may not be representative of all children in Swansea/ Wales. The nested nature of the data (i.e. participants nested within schools) was a limitation common to both Studies 2 and 3 , due to the independence assumption of ANOVAs/ANCOVAs. However, Study 3 did attempt to overcome this by computing an ICC which demonstrated little statistical dependency in the data, suggesting that the largest proportion of variance lay among participants rather than the school clusters. In Study 4, the tasks included in the Dragon Challenge were selected to be a developmentally-appropriate assessment of physical competence for children in developed countries with similar physical education curricular and sport programmes, therefore may limit the generalisability of the tool due to between country and cultural differences. Future studies may wish to evaluate whether the tasks chosen are also valid in jurisdictions with different physical education and sport programmes. In Study 5 an expectation-maximisation algorithm was used to impute missing data and, although this imputation method has previously been validated (Nelwamondo et al., 2007), the lack of a complete data set may have influenced study findings. Furthermore, the measures selected to best predict each latent variable in the model in Study 5 were selected from the measures involved in the Swan-Linx programme, and therefore other quantitative measures (e.g., accelerometer data rather than self-report physical activity) may have increased the strength of the model. Future research may aim to include more objective measures of physical fitness (e.g., $\mathrm{VO}_{2}$ peak assessments) and body composition (e.g., skinfolds and circumferences, or dual-energy X-ray absorptiometry (DEXA), to assess fat and fat-free mass and percentage body fat) and objective assessments of physical activity (e.g., accelerometers). However, capturing this information on large sample sizes would be expensive and time intensive.

\subsection{Future directions}

This thesis indicates that existing health monitoring systems in Wales ought to include health-related fitness tests and the Dragon Challenge. Surveillance and monitoring of physical activity, fitness and physical competence in children and young people, as well as investigations between subgroups (e.g., age, sex, ethnicity, and socio-economic status), should be conducted routinely to analyse trends and inequalities over time. The dissemination of these results and knowledge transfer will assist with informing delivery and assess the impact of health promotion strategies and in- 
vestments.

Overall, greater investment is needed to promote children and young people's physical activity (Study 1), physical fitness (Studies 2 and 3) and physical competence (Studies 1,4 and 5) to reduce the spiralling social and economic burden of inactivity, and so that future generations gain associated health and developmental benefits. Given that components of health-related fitness are related to health in differing ways, focused services and interventions should work to not only improve cardiorespiratory fitness levels, but also muscular strength. An important additional aim should be to close the gap in inequalities, particularly in areas of high deprivation and in girls.

Study 5 set and quantified an ecological framework with the use of the YPAP-M to promote physical competence, revealing multiple potential pathways, regardless of age, weight status and sex, that could inform future interventions. Administrative and policy diagnosis (Gielen et al., 2008; Green et al., 1980; Welk, 1999), including the assessment of resources, budgets and organisations, should be used to refine interventions or promotional strategies that are likely to be effective based on this model. Focused interventions should also integrate process, impact and outcome evaluations to measure the effectiveness of the implemented intervention (Gielen et al., 2008; Green et al., 1980; Welk, 1999).

Future research should investigate the potential of the Dragon Challenge as a measurement tool to indicate levels of physical competence in terms of different contexts, durations and activity settings. Whilst the Dragon Challenge is more inclusive of the constructs of Whitehead's interpretations of physical competence (Whitehead, 2010), it may not provide a complete assessment of physical competence. Specifically, the Dragon Challenge does not reflect physical competence in terms of different varieties of contexts and durations of activities, activity settings (i.e., water, air, ice; Jurbala, 2015; Whitehead, 2010), or different movement forms (i.e., adventure, aesthetic, athletic, competitive, fitness and health, interactional/relational; Whitehead, 2010). However, many land-based measures assume the transferability of movement skills and movement patterns assessed to other contexts (Giblin et al., 2014; Robinson \& Randall, 2017). This may also be the case for the Dragon Challenge, but future studies could investigate the ability of the Dragon Challenge to reflect competence in differing movement forms and activity settings. Finally, the development of measures of the remaining physical literacy domains (affective and cognitive) through a mixed-method approach (e.g., observations, questionnaires, interviews) should also 
be of focus to construct a full physical literacy measurement model.

\subsection{Final comments and reflection}

This thesis successfully investigated the health, fitness, lifestyle and physical competency levels and inequalities of school children in Wales. Study 1 comprised of a collaboration of a group of experts to grade physical activity indicators with the use of surveillance data to provide insight for policies and interventions across many sectors (e.g., health, sport and recreation, education, transportation and land-use planning) across Wales. Whilst an indicator for physical fitness was not included in Study 1, health-related fitness is a key determinant of physical activity and has been shown to be a stronger marker and predictor of health outcomes and future health than physical activity and will therefore be considered in forthcoming report cards. As such, Studies 2 and 3 investigated health-related fitness in children in Wales. In particular, Study 2 highlighted the secular changes in selected health and fitness measures, using data obtained in 2003 and 2013. With the use of a large dataset, Study 3 investigated socioeconomic and sex differences in selected health-related fitness measures to predict inequalities in current and future health outcomes. Given the importance of the development of physical competence in children and young people, and the emphasis placed on physical literacy by Welsh Government (Study 1), Study 4 summarised the work conducted in collaboration with paediatric exercise science academics, practitioners, and professionals from schools and community sport in co-designing a measure of physical competence in children. This process culminated in the Dragon Challenge, which was aligned to physical education and sport, coaching, school and community programmes that aimed to promote physical literacy. The Dragon Challenge offers a dynamic measure of physical competence for formative and summative assessment purposes, as well as for national surveillance, with data collected from a national sample of over 4,300 children in Wales. Moreover, the Dragon Challenge approach was used to develop an ecological model (Study 5) to provide insight for interventions to promote physical competence that can be used by schools, families, communities, practitioners and academics. Overall the results from each of the studies in this thesis can be used to inform the content and delivery of health education interventions and programmes, and to inform future research projects. This thesis advocates for a balance of physical activity (quantity and exposure) and fitness and physical competency (quality and outcome). 


\section{References}

Active Travel (Wales) Act 2013. (2013). Retrieved May 23, 2016, from http://www.legislation.gov.uk/anaw/2013/7/contents/enacted

Ajzen, I. (1985). From intentions to actions: A theory of planned behavior. In J. Kuhl \& J. Beckman (Eds.), Action-control: From cognitionto behavior (pp. 11-39). New York, NY: Springer.

Andersen, L. B., Harro, M., Sardinha, L. B., Froberg, K., Ekelund, U., Brage, S., \& Anderssen, S. A. (2006). Physical activity and clustered cardiovascular risk in children: a cross-sectional study (The European Youth Heart Study). Lancet (London, England), 368(9532), 299-304. https://doi.org/10.1016/S01406736(06)69075-2

Andersen, L. B., Hasselstrøm, H., Grønfeldt, V., Hansen, S. E., \& Karsten, F. (2004). The relationship between physical fitness and clustered risk, and tracking of clustered risk from adolescence to young adulthood: Eight years follw-up in the Danish Youth and Sport Study. International Journal of Behavioral Nutrition and Physical Activity, 1, 4-7. https://doi.org/10.1186/1479-5868-1-6

Andersen, L. B., Riddoch, C., Kriemler, S., \& Hills, A. (2011). Physical activity and cardiovascular risk factors in children. British Journal of Sports Medicine, 45(11), 871-876. https://doi.org/10.1136/bjsports-2011-090333

Ara, I., Moreno, L. A., Leiva, M. T., Gutin, B., Moreno, L. A., Leiva, M. T., ... Gutin, B. (2007). Adiposity , Physical Activity, and Physical Fitness Among Children From Aragon, Spain. Obesity, 15(8), 1918-1924.

Armstrong, N., Tomkinson, G. R., \& Ekelund, U. (2011). Aerobic fitness and its relationship to sport, exercise training and habitual physical activity during youth. British Journal of Sports Medicine, 45(11), 849-858.

https://doi.org/10.1136/bjsports-2011-090200

Artero, E. G., Espaa-Romero, V., Castro-Piñero, J., Ortega, F. B., Suni, J., CastilloGarzon, M. J., \& Ruiz, J. R. (2011). Reliability of field-based fitness tests in youth. International Journal of Sports Medicine, 32(3), 159-169. https://doi.org/10.1055/s-0030-1268488

Artero, E. G., Ruiz, J. R., Ortega, F. B., España-Romero, V., Vicente-Rodríguez, G., 
Molnar, D., ... Gutiérrez, A. (2011). Muscular and cardiorespiratory fitness are independently associated with metabolic risk in adolescents: the HELENA study. Pediatric Diabetes, 12(8), 704-712. https://doi.org/10.1111/j.13995448.2011.00769.x

Aubert, S., Barnes, J. D., Aguilar-Farias, N., Cardon, G., Chang, C.-K., Delisle Nyström, C., ... Tremblay, M. S. (2018). Report Card grades on the physical activity of children and youth comparing 30 very high Human Development Index countries. Journal of Physical Activity and Health. https://doi.org/10.1123/jpah.2018-0431

Babic, M. J., Morgan, P. J., Plotnikoff, R. C., Lonsdale, C., White, R. L., \& Lubans, D. R. (2014). Physical Activity and Physical Self-Concept in Youth:

Systematic Review and Meta-Analysis. Sports Medicine, 44(11), 1589-1601. https://doi.org/10.1007/s40279-014-0229-z

Bailey, L. (2015). Child Measurement Programme for Wales 2013/14. Public Health Wales NHS Trust. Retrieved from http://www.wales.nhs.uk/sitesplus/888/page/67795

Bandura, A. (1986). Social foundations of thought and action: A social-cognitive theory. Englewood Cliffs, NJ: Prentice Hall.

Barnett, L. M., Lai, S. K., Veldman, S. L. C., Hardy, L. L., Cliff, D. P., Morgan, P. J., ... Okely, A. D. (2016). Correlates of Gross Motor Competence in Children and Adolescents : A Systematic Review and Meta-Analysis. Sports Medicine, 46(11), 1663-1688. https://doi.org/10.1007/s40279-016-0495-Z

Barnett, L. M., Morgan, P. J., van Beurden, E., Ball, K., \& Lubans, D. R. (2011). A reverse pathway? Actual and perceived skill proficiency and physical activity. Medicine and Science in Sports and Exercise, 43(5), 898-904. https://doi.org/10.1249/MSS.0b013e3181fdfadd

Barnett, L. M., Morgan, P. J., van Beurden, E., \& Beard, J. R. (2008). Perceived sports competence mediates the relationship between childhood motor skill proficiency and adolescent physical activity and fitness: A longitudinal assessment. International Journal of Behavioral Nutrition and Physical Activity, 5, 1-12. https://doi.org/10.1186/1479-5868-5-40

Barnett, L. M., Stodden, D., Miller, A. D., Cohen, K. E., Smith, J. J., Dudley, D., ... 
Morgan, P. J. (2016). Fundamental Movement Skills : An Important Focus. Journal of Teaching in Physical Education, 35, 219-225.

Barnett, L. M., van Beurden, E., Morgan, P. J., Brooks, L. O., \& Beard, J. R. (2008). Does childhood motor skill proficiency predict adolescent fitness? Medicine and Science in Sports and Exercise, 40(12), 2137-2144. https://doi.org/10.1249/MSS.0b013e31818160d3

Barnett, L. M., van Beurden, E., Morgan, P. J., Brooks, L. O., \& Beard, J. R. (2009). Childhood Motor Skill Proficiency as a Predictor of Adolescent Physical Activity. Journal of Adolescent Health, 44(3), 252-259.

Barnett, L. M., van Beurden, E., Morgan, P. J., Brooks, L. O., \& Beard, J. R. (2010). Gender Differences in Motor Skill Proficiency From Childhood to Adolescence : A Longitudinal Study. Research Quarterly for Exercise and Sport, 81(2), 162-170.

Bauman, A. E., Reis, R. S., Sallis, J. F., Wells, J. C., Loos, R. J. F., Martin, B. W., ... Sarmiento, O. L. (2012). Correlates of physical activity: Why are some people physically active and others not? The Lancet, 380(9838), 258-271. https://doi.org/10.1016/S0140-6736(12)60735-1

Beran, T. N., \& Violato, C. (2010). Structural equation modeling in medical research: A primer. BMC Research Notes, 3(1), 267. https://doi.org/10.1186/1756-0500-3-267

Besson, H., Ekelund, U., Luan, J., May, A. M., Sharp, S., Travier, N., ... Peeters, P. H. (2009). A cross-sectional analysis of physical activity and obesity indicators in European participants of the EPIC-PANACEA study. International Journal of Obesity, 33(4), 497-506. https://doi.org/10.1038/ijo.2009.25

Beth Nesa? What Next? 2015. (2015). Retrieved from https://www.childcomwales.org.uk/wp-content/uploads/2016/04/OverviewEnglish-FINAL.compressed.pdf

Beunen, G. P., Rogol, A. D., \& Malina, R. M. (2006). Indicators of biological maturation and secular changes in biological maturation. Food and Nutrition Bulletin, 27(4 SUPPL.), 244-256. https://doi.org/10.1177/15648265060274S508

Biddle, S. J. H., \& Asare, M. (2011). Physical activity and mental health in children 
and adolescents: A review of reviews. British Journal of Sports Medicine, 45(11), 886-895. https://doi.org/10.1136/bjsports-2011-090185

Blaes, A., Baquet, G., Fabre, C., Van Praagh, E., \& Berthoin, S. (2011). Is there any relationship between physical activity level and patterns, and physical performance in children? International Journal of Behavioral Nutrition and Physical Activity, 8(1), 122. https://doi.org/10.1186/1479-5868-8-122

Blair, S. N., Cheng, Y., \& Holder, J. (2001). Is Physical Activity or Physical Fitness More Important in Defining Health Benefits? Medicine \& Science in Sports \& Exercise, 33(6 Suppl), S379-S399. https://doi.org/10.1097/00005768200105001-01549

Boddy, L. M., Fairclough, S. J., Atkinson, G., \& Stratton, G. (2012). Changes in cardiorespiratory fitness in 9- to 10.9-year-old children: Sportslinx 1998-2010. Medicine and Science in Sports and Exercise, 44(3), 481-486. https://doi.org/10.1249/MSS.0b013e3182300267

Boddy, L. M., Hackett, A. F., \& Stratton, G. (2009). Changes in BMI and prevalence of obesity and overweight in children in Liverpool, 1998-2006. Perspectives in Public Health, 129(3), 127-131. https://doi.org/10.1177/1757913908094808

Boddy, L. M., Hackett, A. F., \& Stratton, G. (2010). Changes in fitness, body mass index and obesity in 9-10 year olds. Journal of Human Nutrition and Dietetics, 23(3), 254-259. https://doi.org/10.1111/j.1365-277X.2009.01025.x

Boddy, L. M., Stratton, G., \& Hackett, A. F. (2010). The test/re-test reliability of a field-based fitness test battery in 9-10 year old schoolchildren. In G. Baquet \& S. Berthoin (Eds.), Children and Exercise XXV: The proceedings of the 25th Pediatric Work Physiology Meeting (pp. 213-218). Routledge.

Boreham, C., \& McKay, H. A. (2011). Physical activity in childhood and bone health. British Journal of Sports Medicine, 45(11), 877-879. https://doi.org/10.1136/bjsports-2011-090188

Boreham, C., \& Riddoch, C. (2001). The physical activity, fitness and health of children. Journal of Sports Sciences, 19(12), 915-929. https://doi.org/10.1080/026404101317108426

Bouchard, C., \& Pérusse, L. (1994). Heredity, activity level, fitness, and health. In 
C. Bouchard, R. J. Shephard, \& T. Stephens (Eds.), Physical Activity, Fitness, and Health: International Proceedings and Consensus Statement (pp. 106118). Champaign, Illinois: Human Kinetics.

Bouchard, C., \& Shephard, R. J. (1994). Physical Activity, Fitness and Health: The Model and Key Concepts. In C. Bouchard, R. J. Shephard, \& T. Stephens (Eds.), Physical Activity, Fitness and Health: International Proceeding and Consensus Statement. Champaign, Illinois: Human Kinetics.

British Heart Foundation National Centre (BHFNC) for Physical Activity and Health Loughborough University. (2012). Sedentary Behaviour: Evidence Briefing. Retrieved from www.bhfactive.org.uk

Brophy, S., Rees, A., Knox, G., Baker, J., \& Thomas, N. E. (2012). Child fitness and father's BMI are important factors in childhood obesity: A school based cross-sectional study. PLoS ONE, 7(5), 1-7.

https://doi.org/10.1371/journal.pone.0036597

Bruininks, R., \& Bruininks, B. (2005). Bruininks-Oseretsky Test of Motor Proficiency, second edition (BOT-2). Minneapolis, MN: Pearson Assessment.

Cairney, J., Veldhuizen, S., Graham, J. D., Rodriguez, C., Bedard, C., Bremer, E., \& Kriellaars, D. (2018). A Construct Validation Study of PLAYfun. Medicine \& Science in Sports \& Exercise, 50(4), 855-862. https://doi.org/10.1249/MSS.0000000000001494

Carson, V., Hunter, S., Kuzik, N., Gray, C. E., Poitras, V. J., Chaput, J.-P., ... Tremblay, M. S. (2016). Systematic review of sedentary behaviour and health indicators in school-aged children and youth: an update 1. Applied Physiology, Nutrition, and Metabolism, 41(6 (Suppl. 3)), 240-265.

https://doi.org/10.1139/apnm-2015-0630

Castro-Piñero, J., Artero, E. G., Espana-Romero, V., Ortega, F. B., Sjostrom, M., Suni, J., \& Ruiz, J. R. (2010). Criterion-related validity of field-based fitness tests in youth: a systematic review. British Journal of Sports Medicine, 44(13), 934-943. https://doi.org/10.1136/bjsm.2009.058321

Cattuzzo, M. T., dos Santos Henrique, R., Ré, A. H. N., Santos de Oliveira, I., Melo, B. M., de Sousa Moura, M., ... Stodden, D. (2016). Motor competence and health related physical fitness in youth: A systematic review. Journal of 
Science and Medicine in Sport, 19(2), 123-129.

https://doi.org/10.1016/j.jsams.2014.12.004

Ceschia, A., Giacomini, S., Santarossa, S., Rugo, M., Salvadego, D., Da Ponte, A., ... Lazzer, S. (2016). Deleterious effects of obesity on physical fitness in prepubertal children. European Journal of Sport Science, 16(2), 271-278. https://doi.org/10.1080/17461391.2015.1030454

Chaput, J.-P., Gray, C. E., Poitras, V. J., Carson, V., Gruber, R., Olds, T., ... Tremblay, M. S. (2016). Systematic review of the relationships between sleep duration and health indicators in school-aged children and youth. Applied Physiology, Nutrition, and Metabolism, 41(6 (Suppl. 3)), 266-282.

https://doi.org/10.1139/apnm-2015-0627

Chaput, J.-P., Klingenberg, L., Rosenkilde, M., Gilbert, J.-A., Tremblay, A., \& Sjödin, A. (2011). Physical Activity Plays an Important Role in Body Weight Regulation. Journal of Obesity, 2011, 1-11. https://doi.org/10.1155/2011/360257

Charlton, R., Gravenor, M. B., Rees, A., Knox, G., Hill, R., Rahman, M. A., ... Brophy, S. (2014). Factors associated with low fitness in adolescents--a mixed methods study. BMC Public Health, 14, 764. https://doi.org/http://dx.doi.org/10.1186/1471-2458-14-764

Chen, S., Welk, G. J., \& Joens-Matre, R. R. (2014). Testing the youth physical activity promotion model: Fatness and fitness as enabling factors.

Measurement in Physical Education and Exercise Science, 18(4), 227-241. https://doi.org/10.1080/1091367X.2014.936017

Cheung, C.-L., Nguyen, U.-S. D. T., Au, E., Tan, K. C. B., \& Kung, A. W. C. (2013). Association of handgrip strength with chronic diseases and multimorbidity. AGE, 35(3), 929-941. https://doi.org/10.1007/s11357-0129385-y

Cheung, G. W., \& Rensvold, R. B. (2002). Evaluating goodness-of-fit indexes for testing measurement invariance, 9(2), 233-255. https://doi.org/10.1207/S15328007SEM0902

Chinapaw, M. J. M., Proper, K. I., Brug, J., van Mechelen, W., \& Singh, A. S. (2011). Relationship between young peoples' sedentary behaviour and 
biomedical health indicators: a systematic review of prospective studies.

Obesity Reviews, 12(7), e621-e632. https://doi.org/10.1111/j.1467789X.2011.00865.X

Clark, J. E., \& Metcalfe, J. S. (2002). Motor Development: Research and Reviews (Vol. 2.). Reston, VA: National Association of Sport and Physical Education. Cleland, V. J., Ball, K., Magnussen, C., Dwyer, T., \& Venn, A. (2009). Socioeconomic position and the tracking of physical activity and cardiorespiratory fitness from childhood to adulthood. American Journal of Epidemiology, 170(9), 1069-1077. https://doi.org/10.1093/aje/kwp271

Cohen, D. D., Voss, C., Taylor, M. J. D., Delextrat, A., Ogunleye, A. A., \& Sandercock, G. R. H. (2011). Ten-year secular changes in muscular fitness in English children. Acta Paediatrica, International Journal of Paediatrics, 100(10), 175-177. https://doi.org/10.1111/j.1651-2227.2011.02318.x

Cohen, D. D., Voss, C., Taylor, M. J. D., Stasinopoulos, D. M., Delextrat, A., \& Sandercock, G. R. H. (2010). Handgrip strength in English schoolchildren. Acta Paediatrica, 99(7), 1065-1072. https://doi.org/10.1111/j.16512227.2010.01723.x

Cohen, J. (1992). A Power Primer. Psychological Bulletin, 112(1), 155-159.

Cole, T. J., Bellizzi, M. C., Flegal, K. M., \& Dietz, W. H. (2000). Establishing a standard definition for child overweight and obesity worldwide: international survey. BMJ, 320, 1-6. https://doi.org/10.1136/bmj.320.7244.1240

Cole, T. J., Freeman, J. V., \& Preece, M. A. (1995). Body mass index reference curves for the UK, 1990. Arch Dis Child, 73, 25-29.

Cools, W., De Martelaer, K., Samaey, C., \& Andries, C. (2011). Fundamental movement skill performance of preschool children in relation to family context. Journal of Sports Sciences, 29(7), 649-660. https://doi.org/10.1080/02640414.2010.551540

Corbin, C. B. (2016). Implications of Physical Literacy for Research and Practice: A Commentary. Research Quarterly for Exercise and Sport, 87(1), 14-27. https://doi.org/10.1080/02701367.2016.1124722

Corbin, C. B., Welk, G. J., Corbin, W. R., \& Welk, K. A. (2006). Concepts of physical fitness: Active lifestyles for wellness (13th ed.). New York, NY: 
McGraw-Hill.

Cote, A. T., Harris, K. C., Panagiotopoulos, C., Sandor, G. G. S., \& Devlin, A. M. (2013). Childhood Obesity and Cardiovascular Dysfunction. Journal of the American College of Cardiology, 62(15), 1309-1319.

https://doi.org/10.1016/j.jacc.2013.07.042

Council of Europe. (1993). Eurofit: Handbook for the Eurofit tests of physical fitness (2nd ed.). Strasbourg: Council of Europe, Committee for the Development of Sport.

CSEP Special Supplement - Canadian 24-Hour Movement Guidelines for Children and Youth: An Integration of Physical Activity, Sedentary Behaviour, and Sleep. (2016). Applied Physiology, Nutrition, and Metabolism, 41(6 (Suppl.

3)), i-i. https://doi.org/10.1139/apnm-2016-0266

D’Hondt, E., Deforche, B., Gentier, I., Verstuyf, J., Vaeyens, R., De Bourdeaudhuij, I., ... Lenoir, M. (2014). A longitudinal study of gross motor coordination and weight status in children. Obesity, 22(6), 1505-1511. https://doi.org/10.1002/oby.20723

Das, P., \& Horton, R. (2012). Rethinking our approach to physical activity. The Lancet, 380(9838), 189-190. https://doi.org/10.1016/S0140-6736(12)61024-1

De Miguel-Etayo, P., Gracia-Marco, L., Ortega, F. B., Intemann, T., Foraita, R., Lissner, L., ... Moreno, L. A. (2014). Physical fitness reference standards in European children: the IDEFICS study. International Journal of Obesity, 38(S2), S57-S66. https://doi.org/10.1038/ijo.2014.136

Deci, E. L., \& Ryan, R. M. (1985). Intrinsic motivation and self-determination in human behavior. New York, NY: Plenum Press.

Department of Health. (2011). Start Active, Stay Active: A report on physical activity from the four home countries' Chief Medical Officers. https://doi.org/https://www.gov.uk/government/uploads/system/uploads/attach ment_data/file/216370/dh_128210.pdf

Dinsdale, H., Ridler, C., \& Ells, L. J. (2011). A simple guide to classifying body mass index in children. Oxford: National Obesity Observatory.

Dragon Challenge Circuit Video. (2015). Retrieved May 23, 2016, from https://www.youtube.com/watch?v=1SPLtwDrgRM 
Dugdill, L., \& Stratton, G. (2009). Evaluating Sport and Physical Activity Interventions. In L. Dugdill, D. Crone, \& R. Murphy (Eds.), Physical Activity and Health Promotion: Evidence Based Approaches to Practice. Blackwell Publishing.

Duger, T., Bumin, G., Uyanik, M., Aki, E., \& Kayihan, H. (1999). The assessment of Bruininks-Oseretsky test of motor proficiency in children. Pediatric Rehabilitation, 3, 125-131.

Dumith, S. C., Gigante, D. P., Domingues, M. R., \& Kohl, H. W. (2011). Physical activity change during adolescence: A systematic review and a pooled analysis. International Journal of Epidemiology, 40(3), 685-698.

https://doi.org/10.1093/ije/dyq272

Dummer, T. J. B., Gibbon, M. A., Hackett, A. F., Stratton, G., \& Taylor, S. R. (2005). Is overweight and obesity in 9-10-year-old children in Liverpool related to deprivation and/or electoral ward when based on school attended? Public Health Nutrition, 8(6), 636-641. https://doi.org/10.1079/PHN2005719

Dwyer, T., Magnussen, C. G., Schmidt, M. D., Ukoumunne, O. C., Ponsonby, A.L., Raitakari, O. T., ... Venn, A. (2009). Decline in Physical Fitness From Childhood to Adulthood Associated With Increased. Diabetes Care, 32(4), 683-687. https://doi.org/10.2337/dc08-1638.

Dziura, J., Kasl, S. V., \& Di Pietro, L. (2004). Physical Activity Reduces Type 2 Diabetes Risk in Aging Independent of Body Weight Change. Journal of Physical Activity \& Health, 1(1), 19-28.

Edwards, L. C., Bryant, A. S., Keegan, R. J., Morgan, K., \& Jones, A. M. (2016). Definitions, Foundations and Associations of Physical Literacy: A Systematic Review. Sports Medicine, 47(1), 1-14. https://doi.org/10.1007/s40279-0160560-7

Eisenmann, J. C., Katzmarzyk, P. T., Perusse, L., Tremblay, A., Després, J. P., \& Bouchard, C. (2005). Aerobic fitness, body mass index, and CVD risk factors among adolescents: The Québec family study. International Journal of Obesity, 29(9), 1077-1083. https://doi.org/10.1038/sj.ijo.0802995

Ekelund, U., Anderssen, S. A., Froberg, K., Sardinha, L. B., Andersen, L. B., \& Brage, S. (2007). Independent associations of physical activity and 
cardiorespiratory fitness with metabolic risk factors in children: the European youth heart study. Diabetologia, 50(9), 1832-1840.

https://doi.org/10.1007/s00125-007-0762-5

Ekelund, U., Brage, S., Froberg, K., Harro, M., Anderssen, S. A., Sardinha, L. B., ... Andersen, L. B. (2006). TV viewing and physical activity are independently associated with metabolic risk in children: The European youth heart study. PLoS Medicine, 3(12), 2449-2457. https://doi.org/10.1371/journal.pmed.0030488

Eston, R., \& Reilly, T. (2009). Kinanthropometry and Exercise Physiology Laboratory Manual: Anthropometry. Vol. 1 (3rd ed.). Routledge.

Fairclough, S. J., Boddy, L. M., Hackett, A. F., \& Stratton, G. (2009). Associations between children's socioeconomic status, weight status, and sex, with screenbased sedentary behaviours and sport participation. International Journal of Pediatric Obesity, 4(4), 299-305. https://doi.org/10.3109/17477160902811215

Field, A. E., Laird, N., Steinberg, E., Fallon, E., Semega-Janneh, M., \& Yanovski, J. A. (2003). Which metric of relative weight best captures body fatness in children? Obesity Research, 11(11), 1345-1352. https://doi.org/10.1038/oby.2003.182

Flatt, J.-P. (2001). Macronutrient Composition and Food Selection. Obesity, 9(11S), 256S-262S. https://doi.org/10.1038/oby.2001.128

Freitas, D., Maia, J., Beunen, G., Claessens, A., Thomis, M., Marques, A., ... Lefevre, J. (2007). Socio-economic status, growth, physical activity and fitness: The Madeira Growth Study. Annals of Human Biology, 34(1), 107122. https://doi.org/10.1080/03014460601080983

Gallahue, D. L., \& Donnelly, F. C. (2007). Developmental Physical Education for All Children (4th ed.). Champaign, IL: Human Kinetics.

Gallahue, D. L., Ozmun, J. C., \& Goodway, J. D. (2012). Understanding motor development: Infants, children, adolescents, adults (7th ed.). New York, NY: McGraw-Hill.

García-Hermoso, A., Cavero-Redondo, I., Ramírez-Vélez, R., Ruiz, J. R., Ortega, F. B., Lee, D.-C., \& Martínez-Vizcaíno, V. (2018). Muscular Strength as a Predictor of All-Cause Mortality in an Apparently Healthy Population: A 
Systematic Review and Meta-Analysis of Data From Approximately 2 Million Men and Women. Archives of Physical Medicine and Rehabilitation, 99(10), 2100-2113. https://doi.org/10.1016/j.apmr.2018.01.008

Garcia, A. W., Broda, M. A. N., Frenn, M., Coviak, C., Pender, N. J., \& Ronis, D. L. (1995). Gender and Developmental Differences in Exercise Beliefs Among Youth and Prediction of Their Exercise Behavior. The Journal of School Health, 65(6), 213-219.

Giblin, S., Collins, D., \& Button, C. (2014). Physical literacy: importance, assessment and future directions. Sports Medicine (Auckland, N.Z.), 44(9), 1177-1184. https://doi.org/10.1007/s40279-014-0205-7

Gielen, A. C., McDonald, E. M., Gary, T. L., \& Bone, L. R. (2008). Using the PRECEDE/PROCEED Model to Apply Health Behavior Theories. In K. Glanz, F. M. B. K. Rimer, \& K. Viswanath (Eds.), Health Behavior and Health Education: Theory, Research and Practice (4th ed., pp. 407-433). San Francisco: Jossey-Bass.

Gillis, L., Tomkinson, G., Olds, T., Moreira, C., Christie, C., Nigg, C., ... Van Mechelen, W. (2013). Research priorities for child and adolescent physical activity and sedentary behaviours: an international perspective using a twinpanel Delphi procedure. The International Journal of Behavioral Nutrition and Physical Activity, 10(1), 112. https://doi.org/10.1186/1479-5868-10-112

Glanz, K., Rimer, B. K., \& Viswanath, K. (2008). Health Behavior and Health Education: Theory, Research, and Practice. (K. Glanz, B. K. Rimer, \& K. Viswanath, Eds.) (4th ed.). San Francisco: Jossey-Bass.

Golle, K., Granacher, U., Hoffmann, M., Wick, D., \& Muehlbauer, T. (2014). Effect of living area and sports club participation on physical fitness in children: a 4 year longitudinal study. BMC Public Health, 14(1), 499. https://doi.org/10.1186/1471-2458-14-499

Green, L. W., Kreuter, M. W., Deeds, S. G., \& Partridge, K. B. (1980). Health education planning: A diagnostic approach. California: Mayfield.

Griffiths, L. J., Parsons, T. J., \& Hill, A. J. (2010). Self-esteem and quality of life in obese children and adolescents: A systematic review. International Journal of Pediatric Obesity, 5(4), 282-304. https://doi.org/10.3109/17477160903473697 
Guinhouya, B. C. (2012). Physical Activity in the Prevention of Childhood Obesity. Paediatric and Perinatal Epidemiology, 26(5), 438-447. https://doi.org/10.1111/j.1365-3016.2012.01269.x

Haines, L., Wan, K. C., Lynn, R., Barret, T. G., \& Shield, J. P. H. (2007). Rising Incidence of Type 2 Diabetes in Children in the U.K. Diabetes Care, 30(5), 1097-1101. https://doi.org/10.2337/dc06-1813.Abbreviations

Hallal, P. C., Andersen, L. B., Bull, F. C., Guthold, R., Haskell, W., Ekelund, U., ... Wells, J. C. (2012). Global physical activity levels: Surveillance progress, pitfalls, and prospects. The Lancet, 380(9838), 247-257. https://doi.org/10.1016/S0140-6736(12)60646-1

Hanson, M. D., \& Chen, E. (2007). Socioeconomic status and health behaviors in adolescence: A review of the literature. Journal of Behavioral Medicine, 30(3), 263-285. https://doi.org/10.1007/s10865-007-9098-3

Hardy, L. L., King, L., Farrell, L., Macniven, R., \& Howlett, S. (2010). Fundamental movement skills among Australian preschool children. Journal of Science and Medicine in Sport, 13(5), 503-508. https://doi.org/10.1016/j.jsams.2009.05.010

Harter, S. (1978). Effectance motivation reconsidered: Toward a developmental model. Human Development, 21, 34-64.

Hay, J. A. (1992). Adequacy in and Predilection for Physical Activity in Children. Clinical Journal of Sport Medicine, 2, 192-201.

Haywood, K. M., \& Getchell, N. (2014). Life Span Motor Development (6th ed.). Champaign, IL: Human Kinetics.

Henderson, S. E., Sugden, D. A., \& Barnett, A. L. (2007). Movement Assessment Battery for Children - second edition (Movement ABC-2); examiner's manual. London: Harcourt Assessment.

Henrique, R. S., Ré, A. H. N., Stodden, D. F., Fransen, J., Campos, C. M. C., Queiroz, D. R., \& Cattuzzo, M. T. (2016). Association between sports participation, motor competence and weight status: A longitudinal study. Journal of Science and Medicine in Sport, 19(10), 825-829. https://doi.org/10.1016/j.jsams.2015.12.512

Hills, A. P., Andersen, L. B., \& Byrne, N. M. (2011). Physical activity and obesity 
in children, 866-870. https://doi.org/10.1136/bjsports-2011-090199

Högström, G., Nordström, A., \& Nordström, P. (2015). Aerobic fitness in late adolescence and the risk of early death: a prospective cohort study of 1.3 million Swedish men. International Journal of Epidemiology, 45(4), 11591168. https://doi.org/10.1093/ije/dyv321

Holfelder, B., \& Schott, N. (2014). Relationship of fundamental movement skills and physical activity in children and adolescents : A systematic review. Psychology of Sport \& Exercise, 15(4), 382-391. https://doi.org/10.1016/j.psychsport.2014.03.005

Hu, L.-T., \& Bentler, P. M. (1999). Cutoff criteria for fit indexes in covariance structure analysis : Conventional criteria versus new alternatives. Structural Equation Modeling: A Multidisciplinary Journal, 6(1), 1-55. https://doi.org/10.1080/10705519909540118

Hulteen, R. M., Morgan, P. J., Barnett, L. M., Stodden, D. F., \& Lubans, D. R. (2018). Development of Foundational Movement Skills: A Conceptual Model for Physical Activity Across the Lifespan. Sports Medicine, 48(7), 1533-40. https://doi.org/10.1007/s40279-018-0892-6

Hume, C., Okely, A., Bagley, S., Telford, A., Booth, M., Crawford, D., \& Salmon, J. (2008). Does Weight Status Influence Associations Between Children's Fundamental Movement Skills and Physical Activity? Research Quarterly for Exercise and Sport, 79(2), 158-165.

https://doi.org/10.1080/02701367.2008.10599479

Inchley, J., Currie, D., Young, T., Samdal, O., Torsheim, T., Augustson, L., ... Barnekow, V. (2016). Health Policy for Children and Adolescents, No. 7. Growing Up Unequal: Gender and Socioeconomic Differences in Young People's Health and Well-being. Health Behaviour in School-aged Children (HBSC) Study: International Report from the 2013/2014 Survey. Copenhagen, Denmark: WHO Regional Office for Europe.

International Physical Literacy Association. (2016). Definition of physical literacy. Retrieved October 19, 2017, from https://www.physical-literacy.org.uk/ Issartel, J., McGrane, B., Fletcher, R., O’Brien, W., Powell, D., \& Belton, S. (2017). A cross-validation study of the TGMD-2: The case of an adolescent 
population. Journal of Science and Medicine in Sport, 20(5), 475-479. https://doi.org/10.1016/j.jsams.2016.09.013

Jaakkola, T., Hillman, C., Kalaja, S., \& Liukkonen, J. (2015). The associations among fundamental movement skills, self-reported physical activity and academic performance during junior high school in Finland. Journal of Sports Sciences, 33(16), 1719-1729.

Jakicic, J. M., \& Davis, K. K. (2011). Obesity and Physical Activity. Psychiatric Clinics of North America, 34(4), 829-840. https://doi.org/10.1016/j.psc.2011.08.009

Janssen, I., \& Leblanc, A. G. (2010). Systematic review of the health benefits of physical activity and fitness in school-aged children and youth. The International Journal of Behavioral Nutrition and Physical Activity, 7, 40. https://doi.org/10.1186/1479-5868-7-40

Janz, K. F., Kwon, S., Letuchy, E. M., Eichenberger Gilmore, J. M., Burns, T. L., Torner, J. C., ... Levy, S. M. (2009). Sustained Effect of Early Physical Activity on Body Fat Mass in Older Children. American Journal of Preventive Medicine, 37(1), 35-40. https://doi.org/10.1016/j.amepre.2009.03.012 Jiménez-Pavón, D., Ortega, F. B., Ruiz, J. R., Chilln, P., Castillo, R., Artero, E. G., ... Gonzalez-Gross, M. (2010). Influence of socioeconomic factors on fitness and fatness in Spanish adolescents: The AVENA study. International Journal of Pediatric Obesity, 5(6), 467-473. https://doi.org/10.3109/17477160903576093

Jurbala, P. (2015). What Is Physical Literacy, Really? Quest, 67(4), 367-383. https://doi.org/10.1080/00336297.2015.1084341

Kaiser, H. F. (1960). The application of electronic computers to factor analysis. Educational and Psychology Measurement, 20, 141-151.

Kaiser, H. F. (1974). An index of factorial simplicity. Psychometrika, 39(1), 31-36. Katzmarzyk, P. T., Shen, W., Baxter-Jones, A., Bell, J. D., Butte, N. F., Demerath, E. W., ... Wells, J. C. K. (2012). Adiposity in children and adolescents: Correlates and clinical consequences of fat stored in specific body depots. Pediatric Obesity, 7(5), 42-61. https://doi.org/10.1111/j.20476310.2012.00073.x 
Kelly, L. A., Reilly, J. J., Jackson, D. M., Montgomery, C., Grant, S., \& Paton, J. Y. (2007). Tracking physical activity and sedentary behavior in young children. Pediatric Exercise Science, 19(1), 51-60. https://doi.org/10.1123/pes.19.1.51

Kohl, H. W., Craig, C. L., Lambert, E. V., Inoue, S., Alkandari, J. R., Leetongin, G., ... Wells, J. C. (2012). The pandemic of physical inactivity: Global action for public health. The Lancet, 380(9838), 294-305. https://doi.org/10.1016/S0140$6736(12) 60898-8$

Kristensen, P. L., Wedderkopp, N., Møller, N. C., Andersen, L. B., Bai, C. N., \& Froberg, K. (2006). Tracking and prevalence of cardiovascular disease risk factors across socio-economic classes: A longitudinal substudy of the European Youth Heart Study. BMC Public Health, 6, 1-9. https://doi.org/10.1186/14712458-6-20

L'Allemand-Jander, D. (2010). Clinical diagnosis of metabolic and cardiovascular risks in overweight children: Early development of chronic diseases in the obese child. International Journal of Obesity, 34, S32-S36. https://doi.org/10.1038/ijo.2010.237

Lebrun, C. E. I., van der Schouw, Y. T., de Jong, F. H., Grobbee, D. E., \& Lamberts, S. W. (2006). Fat mass rather than muscle strength is the major determinant of physical function and disability in postmenopausal women younger than 75 years of age. Menopause, 13(3), 474-481. https://doi.org/10.1097/01.gme.0000222331.23478.ec

Lee, I. M., Shiroma, E. J., Lobelo, F., Puska, P., Blair, S. N., Katzmarzyk, P. T., ... Wells, J. C. (2012). Effect of physical inactivity on major non-communicable diseases worldwide: An analysis of burden of disease and life expectancy. The Lancet, 380(9838), 219-229. https://doi.org/10.1016/S0140-6736(12)61031-9

Léger, L. A., Mercier, D., Gadoury, C., \& Lambert, J. (1988). The multistage 20 metre shuttle run test for aerobic fitness. Journal of Sports Sciences, 6(2), 93101. https://doi.org/10.1080/02640418808729800

Li, J., \& Siegrist, J. (2012). Physical activity and risk of cardiovascular disease-a meta-analysis of prospective cohort studies. International Journal of Environmental Research and Public Health, 9(2), 391-407. https://doi.org/10.3390/ijerph9020391 
Little Voices Shouting Out Report. (2015). Retrieved September 17, 2018, from https://www.lleisiaubach.org/home

Livingstone, B. (2000). Epidemiology of childhood obesity in Europe. European Journal of Pediatrics, 159(S1), S14-S34. https://doi.org/10.1007/PL00014363

Lloyd, M., Saunders, T. J., Bremer, E., \& Tremblay, M. S. (2014). Long-Term Importance of Fundamental Motor Skills: A 20-Year Follow-Up Study. Adapted Physical Activity Quarterly, 31(1), 67-78. https://doi.org/10.1123/apaq.2013-0048

Lloyd, R. S., Oliver, J. L., Faigenbaum, A. D., \& Myer, G. D. (2014).

Chronologicalage vs. biological maturation: implications for exercise programming in youth. The Journal of Strength and Conditioning Research, 28(5), 1454-1464.

Logan, S. W., Barnett, L. M., Goodway, J. D., \& Stodden, D. F. (2017).

Comparison of performance on process- and product-oriented assessments of fundamental motor skills across childhood. Journal of Sports Sciences, 35(7), 634-641. https://doi.org/10.1080/02640414.2016.1183803

Lohman, T. G., \& Going, S. B. (2006). Body composition assessment for development of an international growth standard for preadolescent and adolescent children. Food Nutr Bull, 27(4), 314-325.

Lohman, T. G., Roche, A. F., \& Martorell, R. (1988). Anthropometric Standardization Reference Manual. New York, NY, USA: Wiley. Longmuir, P. E., Boyer, C., Lloyd, M., Borghese, M. M., Knight, E., Saunders, T. J., ... Tremblay, M. S. (2017). Canadian Agility and Movement Skill Assessment (CAMSA): Validity, objectivity, and reliability evidence for children 8-12 years of age. Journal of Sport and Health Science, 6(2), 231240. https://doi.org/10.1016/j.jshs.2015.11.004

Lopes, V. P., Rodrigues, L. P., Maia, J. A., \& Malina, R. M. (2011). Motor coordination as predictor of physical activity in childhood. Scandinavian Journal of Medicine \& Science in Sports, 21(5), 663-669. https://doi.org/10.1111/j.1600-0838.2009.01027.x

Lubans, D. R., Morgan, P. J., Cliff, D. P., Barnett, L. M., \& Okely, A. D. (2010). Fundamental Movement Skills in Children and Adolescents: Review of 
Associated Health Benefits. Sports Medicine, 40(12), 1019-1035.

Lubans, D. R., Smith, J. J., Harries, S. K., Barnett, L. M., \& Faigenbaum, A. D. (2014). Development, test-retest reliability, and construct validity of the resistance training skills battery. Journal of Strength and Conditioning Research / National Strength \& Conditioning Association, 28(5), 1373-1380. https://doi.org/10.1519/JSC.0b013e31829b5527

Lundvall, S. (2015). Physical literacy in the field of physical education - A challenge and a possibility. Journal of Sport and Health Science, 4(2), 113118. https://doi.org/10.1016/j.jshs.2015.02.001

Malina, R. M. (2001). Physical Activity and Fitness: Pathways From Childhood to Adulthood. J. Hum. Biol, 13(July 2000), 162-172. https://doi.org/10.1002/1520-6300(200102/03)13:2<162::AIDAJHB1025>3.0.CO;2-T

Malina, R. M., Bouchard, C., \& Bar-Or, O. (2004). Growth, maturation and physical activity (2nd ed.). Champaign, IL: Human Kinetics.

Malina, R. M., Coelho E Silva, M. J., Figueiredo, A. J., Carling, C., \& Beunen, G. P. (2012). Interrelationships among invasive and non-invasive indicators of biological maturation in adolescent male soccer players. Journal of Sports Sciences, 30(15), 1705-1717. https://doi.org/10.1080/02640414.2011.639382

Malina, R. M., \& Kozieł, S. M. (2014). Validation of maturity offset in a longitudinal sample of Polish girls. Journal of Sports Sciences, 32(14), 13741382. https://doi.org/10.1080/02640414.2014.889846

Malina, R. M., Rogol, A. D., Cumming, S. P., Coelho E Silva, M. J., \& Figueiredo, A. J. (2015). Biological maturation of youth athletes: Assessment and implications. British Journal of Sports Medicine, 49(13), 852-859. https://doi.org/10.1136/bjsports-2015-094623

Mammen, G., \& Faulkner, G. (2013). Physical activity and the prevention of depression: A systematic review of prospective studies. American Journal of Preventive Medicine, 45(5), 649-657. https://doi.org/10.1016/j.amepre.2013.08.001

Marshall, S. J., Biddle, S. J. H., Gorely, T., Cameron, N., \& Murdey, I. (2004). Relationships between media use, body fatness and physical activity in 
children and youth: A meta-analysis. International Journal of Obesity, 28(10), 1238-1246. https://doi.org/10.1038/sj.ijo.0802706

Mayorga-Vega, D., Aguilar-Soto, P., \& Viciana, J. (2015). Criterion-related validity of the 20-m shuttle run test for estimating cardiorespiratory fitness: A metaanalysis. Journal of Sports Science and Medicine, 14(3), 536-547. https://doi.org/10.1016/j.jad.2014.11.061

McAuley, P. A., \& Blair, S. N. (2011). Obesity paradoxes. Journal of Sports Sciences, 29(8), 773-782. https://doi.org/10.1080/02640414.2011.553965

McMurray, R. G., \& Andersen, L. B. (2010). The Influence of Exercise on Metabolic Syndrome in Youth: A Review. American Journal of Lifestyle Medicine, 4(2), 176-186.

https://doi.org/https://doi.org/10.1177/1559827609351234

Melo, X., Santa-Clara, H., Almeida, J. P., Carnero, E. A., Sardinha, L. B., Bruno, P. M., \& Fernhall, B. (2011). Comparing several equations that predict peak VO2using the 20-m multistage-shuttle run-test in 8-10-year-old children. European Journal of Applied Physiology, 111(5), 839-849. https://doi.org/10.1007/s00421-010-1708-z

Metter, E. J., Talbot, L. A., Schrager, M., \& Conwit, R. (2002). Skeletal Muscle Strength as a Predictor of All-Cause Mortality in Healthy Men. J Gerontol A Biol Sci Med Sci, 57(10), B359-365.

Miller, Y. D., \& Dunstan, D. W. (2004). The effectiveness of physical activity interventions for the treatment of overweight and obesity and type 2 diabetes. Journal of Science and Medicine in Sport, 7(1), 52-59. https://doi.org/10.1016/S1440-2440(04)80278-0

Milliken, L. A., Faigenbaum, A. D., Loud, R. L., \& Westcott, W. L. (2008). Correlates of Upper and Lower Body Muscular Strength in Children. Journal of Strength and Conditioning Research, 22(4), 1339-1346. https://doi.org/10.1519/JSC.0b013e31817393b1

Minatto, G., Barbosa Filho, V. C., Berria, J., \& Petroski, E. L. (2016). School-Based Interventions to Improve Cardiorespiratory Fitness in Adolescents: Systematic Review with Meta-analysis. Sports Medicine, 46(9), 1273-1292. https://doi.org/10.1007/s40279-016-0480-6 
Mirwald, R. L., Baxter-Jones, A. D., Bailey, D. A., \& Beunen, G. P. (2002). An assessment of maturity from anthropometric measurements. Medicine $\&$ Science in Sports \& Exercise, 34(4), 689-694.

https://doi.org/10.1097/00005768-200204000-00020

Mokkink, L. B., Terwee, C. B., Patrick, D. L., Alonso, J., Stratford, P. W., Knol, D. L., ... De Vet, H. C. W. (2010). The COSMIN checklist for assessing the methodological quality of studies on measurement properties of health status measurement instruments: An international Delphi study. Quality of Life Research, 19(4), 539-549. https://doi.org/10.1007/s11136-010-9606-8

Moliner-Urdiales, D., Ruiz, J. R., Ortega, F. B., Jiménez-Pavón, D., VicenteRodriguez, G., Rey-López, J. P., ... Moreno, L. A. (2010). Secular trends in health-related physical fitness in Spanish adolescents: The AVENA and HELENA Studies. Journal of Science and Medicine in Sport, 13(6), 584-588. https://doi.org/10.1016/j.jsams.2010.03.004

Monasta, L., Batty, G. D., Cattaneo, A., Lutje, V., Ronfani, L., Van Lenthe, F. J., \& Brug, J. (2010). Early-life determinants of overweight and obesity: a review of systematic reviews. Obesity Reviews, 11(10), 695-708. https://doi.org/10.1111/j.1467-789X.2010.00735.x

Moore, L. L., Gao, D., Bradlee, M. L., Cupples, L. A., Sundarajan-Ramamurti, A., Proctor, M. H., ... Ellison, R. C. (2003). Does early physical activity predict body fat change throughout childhood? Preventive Medicine, 37(1), 10-17. https://doi.org/10.1016/S0091-7435(03)00048-3

Morales, P. F., Sánchez-López, M., Moya-Martínez, P., García-Prieto, J. C., Martínez-Andrés, M., García, N. L., \& Martínez-Vizcaíno, V. (2013). Healthrelated quality of life, obesity, and fitness in schoolchildren: The Cuenca study. Quality of Life Research, 22(7), 1515-1523. https://doi.org/10.1007/s11136012-0282-8

Mutunga, M., Gallagher, A. M., Boreham, C., Watkins, D. C., Murray, L. J., Cran, G., \& Reilly, J. J. (2006). Socioeconomic differences in risk factors for obesity in adolescents in Northern Ireland. International Journal of Pediatric Obesity, l(2), 114-119. https://doi.org/10.1080/17477160600569560

National Obesity Observatory. (2015). [ARCHIVED CONTENT] UK prevalence : 
Public Health England Obesity Knowledge and Intelligence team. Retrieved September 17, 2018, from

http://webarchive.nationalarchives.gov.uk/20170110171036/https://www.noo.o rg.uk/NOO_about_obesity/child_obesity/UK_prevalence

Nelwamondo, F. V., Mohamed, S., \& Marwala, T. (2007). Missing data: A comparison of neural network and expectation maximization techniques. Current Science, 93(11), 1514-1521. https://doi.org/10.2307/24099079

Nevill, A. M., Duncan, M. J., Lahart, I. M., \& Sandercock, G. (2017). Cardiorespiratory fitness and activity explains the obesity-deprivation relationship in children. Health Promotion International, 1-9. https://doi.org/10.1093/heapro/daw106

Newell, K. (1986). Constraints on the development of coordination. In M. G. Wade \& H. T. Whiting (Eds.), Motor Development in Children: Aspects of Coordination and Control (pp. 341-360). Dordrecht, Netherlands: Martinus Nijhoff. https://doi.org/10.1007/978-94-009-4460-2_19

NICE | The National Institute for Health and Care Excellence. (n.d.). Retrieved May 23, 2016, from https://www.nice.org.uk/

Noble, M., Wright, G., Smith, G., \& Dibben, C. (2006). Measuring multiple deprivation at the small-area level. Environment and Planning A, 38(1), 169185. https://doi.org/10.1068/a37168

Noonan, R. J., Boddy, L. M., Knowles, Z. R., \& Fairclough, S. J. (2016). Crosssectional associations between high-deprivation home and neighbourhood environments, and health-related variables among Liverpool children. $B M J$ Open, 6(1), 1-11. https://doi.org/10.1136/bmjopen-2015-008693

Noonan, R. J., Boddy, L. M., Knowles, Z. R., \& Fairclough, S. J. (2017). Fitness, Fatness and Active School Commuting among Liverpool Schoolchildren. International Journal of Environmental Research and Public Health, 14(995), 1-12. https://doi.org/10.3390/ijerph14090995

NSW Department of Education and Training. (2000). Get skilled: Get active. A K-6 resource to support the teaching of fundamental movement skills. Ryde, NSW: NSW Department of Education and Training.

Oldridge, N. B. (2008). Economic burden of physical inactivity: healthcare costs 
associated with cardiovascular disease. European Journal of Cardiovascular Prevention \& Rehabilitation, 15(2), 130-139.

https://doi.org/10.1097/HJR.0b013e3282f19d42

Olds, T., Maher, C., Zumin, S., Péneau, S., Lioret, S., Castetbon, K., ...

Summerbell, C. (2011). Evidence that the prevalence of childhood overweight is plateauing: data from nine countries. International Journal of Pediatric Obesity, 6(5-6), 342-360. https://doi.org/10.3109/17477166.2011.605895

Olds, T., Tomkinson, G., Léger, L., \& Cazorla, G. (2006). Worldwide variation in the performance of children and adolescents: An analysis of 109 studies of the 20-m shuttle run test in 37 countries. Journal of Sports Sciences, 24(10), 10251038. https://doi.org/10.1080/02640410500432193

Ortega, F. B., Labayen, I., Ruiz, J. R., Kurvinen, E., Loit, H. M., Harro, J., ... Sjöström, M. (2011). Improvements in fitness reduce the risk of becoming overweight across puberty. Medicine and Science in Sports and Exercise, 43(10), 1891-1897. https://doi.org/10.1249/MSS.0b013e3182190d71

Ortega, F. B., Ruiz, J. R., Castillo, M. J., \& Sjöström, M. (2008). Physical fitness in childhood and adolescence: a powerful marker of health. International Journal of Obesity, 32, 1-11. https://doi.org/10.1038/sj.ijo.0803774

Ortega, F. B., Ruiz, J. R., Labayen, I., Hurtig-Wennlöf, A., Harro, J., Kwak, L., ... Sjöström, M. (2013). Role of socio-cultural factors on changes in fitness and adiposity in youth: A 6-year follow-up study. Nutrition, Metabolism and Cardiovascular Diseases, 23(9), 883-890. https://doi.org/10.1016/j.numecd.2012.05.008

Ortega, F. B., Silventoinen, K., Tynelius, P., \& Rasmussen, F. (2012). Muscular strength in male adolescents and premature death: Cohort study of one million participants. BMJ (Online), 345(7884), 1-12.

https://doi.org/10.1136/bmj.e7279

Paalanne, N. P., Korpelainen, R. I., Taimela, S. P., Auvinen, J. P., Tammelin, T. H., Hietikko, T. M., ... Karppinen, J. I. (2009). Muscular fitness in relation to physical activity and television viewing among young adults. Medicine and Science in Sports and Exercise, 41(11), 1997-2002. https://doi.org/10.1249/MSS.0b013e3181a7f3a6 
Parfitt, G., Pavey, T., \& Rowlands, A. V. (2009). Children's physical activity and psychological health: the relevance of intensity. Acta Paediatrica, 98(6), 10371043. https://doi.org/10.1111/j.1651-2227.2009.01255.x

Park, M. H., Falconer, C., Viner, R. M., \& Kinra, S. (2012). The impact of childhood obesity on morbidity and mortality in adulthood: A systematic review. Obesity Reviews, 13(11), 985-1000. https://doi.org/10.1111/j.1467789X.2012.01015.X

Pate, R. R., O’Neill, J. R., \& Lobelo, F. (2008). The Evolving Definition of “Sedentary.” Exercise and Sport Sciences Reviews, 36(4), 173-178. https://doi.org/10.1097/JES.0b013e3181877d1a

Paterson, D. H., \& Warburton, D. E. R. (2010). Physical activity and functional limitations in older adults: A systematic review related to Canada's Physical Activity Guidelines. International Journal of Behavioral Nutrition and Physical Activity, 7(38).

Physical Activity and Nutrition Network | Fitness and Health Info Resources. (n.d.). Retrieved May 23, 2016, from http://www.physicalactivityandnutritionwales.org.uk/

Physical and Health Education Canada (PHE Canada). (2014). Development of Passport for Life. Physical and Health Education Journal, 80(2), 18-21.

Pienaar, A. E., van Reenen, I., \& Weber, A. M. (2017). Sex differences in fundamental movement skills of a selected group of 6-year-old South African children. Early Child Development and Care, 186(12), 1994-2008. https://doi.org/10.1080/03004430.2016.1146263

Play Wales | Chwarae Cymru. (n.d.). Retrieved May 23, 2016, from http://www.playwales.org.uk/eng/

Plowman, S. A., \& Meredith, M. D. (Eds.). (2013). Fitnessgram/Activitygram Reference Guide (4th ed.). Dallas, TX: The Cooper Institute. https://doi.org/10.1055/s-0033-1334967

Poitras, V. J., Gray, C. E., Borghese, M. M., Carson, V., Chaput, J.-P., Janssen, I., ... Tremblay, M. S. (2016). Systematic review of the relationships between objectively measured physical activity and health indicators in school-aged children and youth. Applied Physiology, Nutrition, and Metabolism, 41(6 
(Suppl. 3)), 197-239. https://doi.org/10.1139/apnm-2015-0663

Portney, L. G., \& Watkins, M. P. (1993). Foundations of clinical research:

Applications to practice. Norwalk, CT: Appleton \& Lange.

Public Health Wales. (n.d.). Welsh Network of Healthy School Schemes. Retrieved May 23, 2016, from

http://www.wales.nhs.uk/sitesplus/888/page/82249\#introduction

Public Health Wales Observatory. (2013). Health of Children and Young People in Wales. Carmarthen, UK: Public Health Wales NHS Trust. Retrieved from http://www.wales.nhs.uk/sitesplus/922/page/69313

Reilly, J. J., Methven, E., Mcdowell, Z. C., Hacking, B., Alexander, D., Stewert, L., \& Kelnar, C. J. H. (2003). Health Consequences of Obesity. Arch Dis Child, 88(1), 748-752. https://doi.org/10.1002/pbc.23373

Riddoch, C. (1990). The Northern Ireland health and fitness survey-1989: the fitness, physical activity, attitudes and lifestyles of Northern Ireland postprimary schoolchildren. Belfast, UK: The Queen's University of Belfast.

Robinson, D. B., \& Randall, L. (2017). Marking Physical Literacy or Missing the Mark on Physical Literacy? A Conceptual Critique of Canada's Physical Literacy Assessment Instruments. Measurement in Physical Education and Exercise Science, 21(1), 40-55.

https://doi.org/10.1080/1091367X.2016.1249793

Robinson, L. E., Stodden, D. F., Barnett, L. M., Lopes, V. P., Logan, S. W., Rodrigues, L. P., \& D'Hondt, E. (2015). Motor Competence and its Effect on Positive Developmental Trajectories of Health. Sports Medicine, 45(9), 12731284. https://doi.org/10.1007/s40279-015-0351-6

Rodrigues, L. P., Stodden, D. F., \& Lopes, V. P. (2015). Developmental pathways of change in fitness and motor competence are related to overweight and obesity status at the end of primary school. Journal of Science and Medicine in Sport, 19, 87-92. https://doi.org/10.1016/j.jsams.2015.01.002

Rosseel, Y. (2012). lavaan: An R Package for Structural Equation Modelling. Journal of Statistical Software, 48(2), 1-36. Retrieved from http://www.jstatsoft.org/v48/i02/

Rudd, J., Barnett, L. M., Butson, M. L., Farrow, D., Berry, J., \& Polman, R. C. J. 
(2015). Fundamental movement skills are more than run, throw and catch: The role of stability skills. PLOS ONE, 10(10), 1-15.

https://doi.org/10.1371/journal.pone.0140224

Rudd, J., Butson, M. L., Barnett, L. M., Farrow, D., Berry, J., Borkoles, E., \& Polman, R. (2016). A holistic measurement model of movement competency in children. Journal of Sports Sciences, 34(5), 477-485.

https://doi.org/10.1080/02640414.2015.1061202

Ruiz, J. R., Castro-Piñero, J., Artero, E. G., Ortega, F. B., Sjostrom, M., Suni, J., \& Castillo, M. J. (2009). Predictive validity of health-related fitness in youth: a systematic review. British Journal of Sports Medicine, 43(12), 909-923. https://doi.org/10.1136/bjsm.2008.056499

Ruiz, J. R., Castro-Piñero, J., España-Romero, V., Artero, E. G., Ortega, F. B., Cuenca, M. M., ... Castillo, M. J. (2011). Field-based fitness assessment in young people: the ALPHA health-related fitness test battery for children and adolescents. British Journal of Sports Medicine, 45(6), 518-524. https://doi.org/10.1136/bjsm.2010.075341

Ruiz, J. R., Ortega, F. B., Gutierrez, A., Meusel, D., Sjöström, M., \& Castillo, M. J. (2006). Health-related fitness assessment in childhood and adolescence: a European approach based on the AVENA, EYHS and HELENA studies. Journal of Public Health, 14(5), 269-277. https://doi.org/10.1007/s10389-006$0059-\mathrm{z}$

Ruiz, J. R., Silva, G., Oliveira, N., Ribeiro, J. C., Oliveira, J. F., \& Mota, J. (2009). Criterion-related validity of the 20-m shuttle run test in youths aged 13-19 years. Journal of Sports Sciences, 27(9), 899-906. https://doi.org/10.1080/02640410902902835

Sallis, J. F., Bull, F., Guthold, R., Heath, G. W., Inoue, S., Kelly, P., ... Hallal, P. C. (2016). Progress in physical activity over the Olympic quadrennium. The Lancet, 388(10051), 1325-1336. https://doi.org/10.1016/S01406736(16)30581-5

Sandercock, G., Voss, C., McConnell, D., \& Rayner, P. (2010). Ten year secular declines in the cardiorespiratory fitness of affluent English children are largely independent of changes in body mass index. Archives of Disease in Childhood, 
95(1), 46-47. https://doi.org/10.1136/adc.2009.162107

Sattelmair, J., Pertman, J., Ding, E. L., Kohl, H. W., Haskell, W., \& Lee, I. M.

(2011). Dose-Response Between Physical Activity and Risk of Coronary Heart

Disease: A Meta-Analysis. Circulation, 124(7), 789-795.

https://doi.org/10.1161/CIRCULATIONAHA.110.010710.Dose-Response

Saunders, T. J., Gray, C. E., Poitras, V. J., Chaput, J.-P., Janssen, I., Katzmarzyk, P.

T., ... Carson, V. (2016). Combinations of physical activity, sedentary

behaviour and sleep: relationships with health indicators in school-aged

children and youth. Applied Physiology, Nutrition, and Metabolism, 41(6

(Suppl. 3)), 283-293. https://doi.org/10.1139/apnm-2015-0626

Schoemaker, M. M., Niemeijer, A. S., Flapper, B. C. T., \& Smits-Engelsman, B. C.

M. (2012). Validity and reliability of the Movement Assessment Battery for

Children-2 Checklist for children with and without motor impairments.

Developmental Medicine and Child Neurology, 54(4), 368-375.

https://doi.org/10.1111/j.1469-8749.2012.04226.x

Schuch, F. B., Vancampfort, D., Richards, J., Rosenbaum, S., Ward, P. B., \&

Stubbs, B. (2016). Exercise as a treatment for depression: A meta-analysis

adjusting for publication bias. Journal of Psychiatric Research, 77, 42-51.

https://doi.org/10.1016/j.jpsychires.2016.02.023

Sheldrick, M., Tyler, R., Mackintosh, K., \& Stratton, G. (2018). Relationship

between Sedentary Time, Physical Activity and Multiple Lifestyle Factors in

Children. Journal of Functional Morphology and Kinesiology, 3(1), 15.

https://doi.org/10.3390/jfmk3010015

Shephard, R. J., Allen, C., Benade, A. J., Davies, C. T., Di Prampero, P. E., Hedman, R., ... Simmons, R. (1968). The maximum oxygen intake. An international reference standard of cardiorespiratory fitness. Bulletin of the World Health Organization, 38(5), 757-764.

Sherar, L. B., Cumming, S. P., Eisenmann, J. C., \& Malina, R. M. (2010).

Adolescent Biological Maturity and Physical Activity : Biology Meets Behavior, 22, 332-349.

Sherar, L. B., Griew, P., Esliger, D. W., Cooper, A. R., Ekelund, U., Judge, K., \& Riddoch, C. (2011). International children's accelerometry database (ICAD): 
Design and methods. BMC Public Health, 11(1), 485.

https://doi.org/10.1186/1471-2458-11-485

Shrewsbury, V., \& Wardle, J. (2008). Socioeconomic status and adiposity in childhood: A systematic review of cross-sectional studies 1990-2005. Obesity, 16(2), 275-284. https://doi.org/10.1038/oby.2007.35

Sigmundsson, H., Lorås, H., \& Haga, M. (2016). Assessment of Motor Competence Across the Life Span: Aspects of Reliability and Validity of a New Test Battery. SAGE Open, 6(1), 1-10. https://doi.org/10.1177/2158244016633273

Silverman, I. W. (2011). The secular trend for grip strength in Canada and the United States The secular trend for grip strength in Canada and the United States, (May 2015), 37-41. https://doi.org/10.1080/02640414.2010.547209

Smith, J. J., Eather, N., Morgan, P. J., Plotnikoff, R. C., Faigenbaum, A. D., \& Lubans, D. R. (2014). The health benefits of muscular fitness for children and adolescents: A systematic review and meta-analysis. Sports Medicine, 44(9), 1209-1223. https://doi.org/10.1007/s40279-014-0196-4

Sport Wales. (n.d.-a). Physical Literacy | A Journey Through Life. Retrieved May 12, 2016, from http://physicalliteracy.sportwales.org.uk/en/

Sport Wales. (n.d.-b). Review of Dragon Sport 2010. Retrieved August 11, 2016, from http://sport.wales/research--policy/tools-andresources/publications.aspx\#2010pub

Sport Wales. (n.d.-c). School Sport Survey 2015. Increased probability of being 'hooked on sport.' Retrieved May 23, 2016, from http://sport.wales/media/1194537/theme2.pdf

Sport Wales. (n.d.-d). Sport Wales Vision. Retrieved September 17, 2018, from http://sport.wales/about-us/about-sport-wales/vision-for-sport.aspx

Sport Wales. (2011). “5x60” Case Studies 2011. Retrieved August 11, 2016, from http://sport.wales/research--policy/tools-andresources/publications.aspx\#2011pub

Sport Wales. (2015a). Active Adult Survey 2014. Retrieved from http://sport.wales/research--policy/surveys-and-statistics/active-adultssurvey.aspx

Sport Wales. (2015b). Further Education Sport Survey 2015. Retrieved from 
http://sport.wales/media/1667685/further_education_sport_survey__state_of_the_nation_2015_english_final_2.pdf

Sport Wales. (2015c). School Sport Survey 2015. Retrieved from

http://sport.wales/media/1667736/school_sport_survey_2015_-

_state_of_the_nation_english.pdf

Statistics Wales. (n.d.). Mid Year Estimates of the Population. Retrieved May 12, 2016, from https://gov.wales/docs/statistics/2008/081204sb702008en.pdf

Stodden, D. F., Gao, Z., Goodway, J. D., \& Langendorfer, S. J. (2014). Dynamic

Relationships Between Motor Skill Competence and Health-Related Fitness in Youth. Pediatric Exercise Science, 26, 231-241.

Stodden, D. F., Goodway, J. D., Langendorfer, S. J., Roberton, M. A., Rudisill, M. E., Garcia, C., \& Garcia, L. E. (2008). A Developmental Perspective on the Role of Motor Skill Competence in Physical Activity : An Emergent Relationship. Quest, 60, 290-306. https://doi.org/https://doi.org/10.1080/00336297.2008.10483582

Stratton, G., Canoy, D., Boddy, L. M., Taylor, S. R., Hackett, A. F., \& Buchan, I. E. (2007). Cardiorespiratory fitness and body mass index of 9-11-year-old English children: a serial cross-sectional study from 1998 to 2004. International Journal of Obesity, 31(7), 1172-1178. https://doi.org/10.1038/sj.ijo.0803562

Stratton, G., Foweather, L., \& Hughes, H. (2017). Dragon Challenge: A National Indicator for Children's Physical Literacy in Wales. Surveillance Report January 2017. Cardiff: Sport Wales.

Stratton, G., Williams, C., Taylor, S. R., Jones, A. M., Mackintosh, K. A., Frost, M., ... Williams, S. (2014). Active Healthy Kids Wales Report Card 2014. Wales.

Stuck, A. E., Walthert, J. M., Nikolaus, T., Büla, C. J., Hohmann, C., \& Beck, J. C. (1999). Risk factors for functional status decline in community-living elderly people: a systematic literature review. Social Science \& Medicine, 48(4), 445469. https://doi.org/10.1016/S0277-9536(98)00370-0

Sustrans Wales, Sustrans Cymru. (n.d.). Retrieved May 23, 2016, from https://www.sustrans.org.uk/wales/our-work-wales

Taylor, A. H., Cable, N. T., Faulkner, G., Hillsdon, M., Narici, M., \& Van der Bij, 
A. K. (2004). Physical activity and older adults: A review of health benefits and the effectiveness of interventions. Journal of Sports Sciences, 22(8), 703725. https://doi.org/10.1080/02640410410001712421

Taylor, S. R., Hackett, A., Stratton, G., \& Lamb, L. (2004). SportsLinx: Improving the Health and Fitness of Liverpool's Youth. Education and Health, 22(1), 1115 .

Telama, R. (2009). Tracking of Physical Activity from Childhood to Adulthood: A Review. Obesity Facts, 2(3), 187-195. https://doi.org/10.1159/000222244

Telama, R., Yang, X., Leskinen, E., Kankaanpää, A., Hirvensalo, M., Tammelin, T., ... Raitakari, O. T. (2014). Tracking of physical activity from early childhood through youth into adulthood. Medicine and Science in Sports and Exercise, 46(5), 955-962. https://doi.org/10.1249/MSS.0000000000000181

Tomkinson, G. R., Carver, K. D., Atkinson, F., Daniell, N. D., Lewis, L. K., Fitzgerald, J. S., ... Ortega, F. B. (2017). European normative values for physical fitness in children and adolescents aged 9-17 years: results from 2 779165 Eurofit performances representing 30 countries. British Journal of Sports Medicine, 0, 1-13. https://doi.org/10.1136/bjsports-2017-098253

Tomkinson, G. R., Lang, J. J., \& Tremblay, M. S. (2017). Temporal trends in the cardiorespiratory fitness of children and adolescents representing 19 highincome and upper middle-income countries between 1981 and 2014. British Journal of Sports Medicine, 0, 1-10. https://doi.org/10.1136/bjsports-2017097982

Tomkinson, G. R., Lang, J. J., Tremblay, M. S., Dale, M., LeBlanc, A. G., Belanger, K., ... Léger, L. (2017). International normative $20 \mathrm{~m}$ shuttle run values from 1 142026 children and youth representing 50 countries. British Journal of Sports Medicine, 51(21), 1545-1554. https://doi.org/10.1136/bjsports-2016-095987

Tomkinson, G. R., Léger, L. A., Olds, T. S., \& Cazorla, G. (2003). Secular Trends in the Performance of Children and Adolescents (1980-2000). Journal of Sports Sciences, 33(4), 285-300. https://doi.org/0112-1642/03/0004$0285 / \$ 30.00 / 0$

Tomkinson, G. R., \& Olds, T. S. (2007). Secular Changes in Pediatric Aerobic Fitness Test Performance: The Global Picture. In Med Sport Sci. (Ed.), 
Pediatric Fitness. Secular Trends and Geographic Variability (Vol 50, pp. 4666). Basel, Switzerland: Karger.

Tomkinson, G. R., \& Olds, T. S. (2008). Field tests of fitness. In N. Armstrong \& W. van Mechelen (Eds.), Paediatric exercise science and medicine (pp. 109128). New York, NY: Oxford University Press.

Tremblay, M. S., Carson, V., \& Chaput, J.-P. (2016). Introduction to the Canadian 24-Hour Movement Guidelines for Children and Youth: An Integration of Physical Activity, Sedentary Behaviour, and Sleep 1. Applied Physiology, Nutrition, and Metabolism, 41(6 (Suppl. 3)), iii-iv.

https://doi.org/10.1139/apnm-2016-0203

Tremblay, M. S., Carson, V., Chaput, J.-P., Connor Gorber, S., Dinh, T., Duggan, M., ... Zehr, L. (2016). Canadian 24-Hour Movement Guidelines for Children and Youth: An Integration of Physical Activity, Sedentary Behaviour, and Sleep 1. Applied Physiology, Nutrition, and Metabolism, 41 (6 (Suppl. 3)), 311327.

Tremblay, M. S., Colley, R. C., Saunders, T. J., Healy, G. N., \& Owen, N. (2010). Physiological and health implications of a sedentary lifestyle. Applied Physiology, Nutrition, and Metabolism, 35(6), 725-740.

https://doi.org/10.1139/H10-079

Tremblay, M. S., Gonzalez, S. A., Katzmarzyk, P. T., Onywera, V. O., Reilly, J. J., \& Tomkinson, G. (2016). Global Matrix 2.0: Report Card Grades on the Physical Activity of Children and Youth Comparing 38 Countries. Journal of Physical Activity and Health, 13(11 Suppl 2), S85-S86.

https://doi.org/10.1123/jpah.2016-0641

Tremblay, M. S., Gray, C. E., Akinroye, K., Harrington, D. M., Katzmarzyk, P. T., Lambert, E. V., ... Tomkinson, G. (2014). Physical Activity of Children: A Global Matrix of Grades Comparing 15 Countries. Journal of Physical Activity and Health, 11(s1), S113-S125. https://doi.org/10.1123/jpah.2014-0177

Tremblay, M. S., LeBlanc, A. G., Kho, M. E., Saunders, T. J., Larouche, R., Colley, R. C., ... Gorber, S. C. (2011). Systematic review of sedentary behaviour and health indicators in school-aged children and youth. International Journal of Behavioral Nutrition and Physical Activity, 8(1), 98. 
https://doi.org/10.1186/1479-5868-8-98

Tremblay, M. S., Shields, M., Laviolette, M., Craig, C. L., Janssen, I., \& Connor Gorber, S. (2010). Fitness of Canadian children and youth: Results from the 2007-2009 Canadian Health Measures Survey. Health Reports, 21(82), 1-15. https://doi.org/82-003-XPE

Triandis, H. C. (1977). Interpersonal behavior. Monterey, CA: Brooks/Cole.

Twisk, J. W. R., Kemper, H. C. G., \& Van Mechelen, W. (2009). Tracking of activity and fitness and the relationship with cardiovascular disease risk factors. Medicine \& Science in Sports \& Exercise, 32(8), 1455-1461.

Tyler, R., Foweather, L., Mackintosh, K. A., \& Stratton, G. (2018). A Dynamic Assessment of Children's Physical Competence. Medicine \& Science in Sports \& Exercise, 50(12), 2474-2487. https://doi.org/10.1249/MSS.0000000000001739

Tyler, R., Mackintosh, K., Brophy, S., Christian, D., Todd, C., Tuvey, S., ... Stratton, G. (2015). Swan-Linx: Fitness Fun Day Report - Swansea Schools (2015). Swansea, U.K. https://doi.org/https://www.swansea.ac.uk/media/SwanLinx\%20Swansea\%20Schools\%\%20Fitness\%20Fun\%20Day\%20feedback\%20r eport\%20(2015).pdf

Tyler, R., Mackintosh, K., Palmer, A., Jones, A., \& Stratton, G. (2015). Ten-Year Secular Changes in Selected Health and Fitness Parameters of 10-11 Years Old Swansea School Children - 2003-2013. Advances in Obesity, Weight Management \& Control, 3(5), 8-13. https://doi.org/10.15406/aowmc.2015.03.00072

Tyler, R., Mannello, M., Mattingley, R., Roberts, C., Sage, R., Taylor, S. R., ... Stratton, G. (2016). Results From Wales' 2016 Report Card on Physical Activity for Children and Youth: Is Wales Turning the Tide on Children's Inactivity? Journal of Physical Activity and Health, 13(11 Suppl 2), S330S336. https://doi.org/10.1123/jpah.2016-0309

UKActive. (2014). Turning the tide of inactivity Acknowledgments, 48. Retrieved from

http://ukactive.com/downloads/managed/Turning_the_tide_of_inactivity.pdf Ulrich, D. A. (2000). TGMD-2: Test of Gross Motor Development. (2nd ed.). 
Austin, Texas: PRO-ED.

United Nations Convention on the Rights of Children - Rights - Children in Wales. (n.d.). Retrieved May 12, 2016, from http://www.childreninwales.org.uk/ourwork/rights/

Van der Fels, I. M. J., te Wierike, S. C. M., Hartman, E., Elferink-Gemser, M. T., Smith, J., \& Visscher, C. (2015). The relationship between motor skills and cognitive skills in 4-16 year old typically developing children: A systematic review. Journal of Science and Medicine in Sport, 18(6), 697-703. https://doi.org/10.1016/j.jsams.2014.09.007

van Jaarsveld, C. H. M., \& Gulliford, M. C. (2015). Childhood obesity trends from primary care electronic health records in England between 1994 and 2013: population-based cohort study. Archives of Disease in Childhood, 100(3), 214219. https://doi.org/10.1136/archdischild-2014-307151

Vlahov, E., Baghurst, T. M., \& Mwavita, M. (2014). Preschool Motor Development Predicting High School Health-Related Physical Fitness: A Prospective Study. Perceptual and Motor Skills, 119(1), 279-291. https://doi.org/10.2466/10.25.PMS.119c16z8

Volaklis, K. A., Halle, M., \& Meisinger, C. (2015). Muscular strength as a strong predictor of mortality: A narrative review. European Journal of Internal Medicine, 26(5), 303-310. https://doi.org/10.1016/j.ejim.2015.04.013

Wales Audit Office. (2007). Increase Physical Activity 2007. Retrieved from http://www.wales.nhs.uk/documents/physical_activity_eng.pdf

Wales UNCRC Monitoring Group. (2015). Report to the United Nations Committee on the Rights of the Child. Retrieved from http://www.savethechildren.org.uk/resources/online-library/rights-here-rightnow

Wang, L., Fan, X., \& Wilson, V. L. (1996). Effects of non-normal data on parameter estimates and fit indices for a model with latent and manifest variables: an empirical study. Structural Equation Modeling A Multidisciplinary Journal, 3(3), 228-247.

Warburton, D. E. R., Charlesworth, S., Ivey, A., Nettlefold, L., \& Bredin, S. S. (2010). A systematic review of the evidence for Canada's Physical Activity 
Guidelines for Adults. https://doi.org/10.14288/1.0223098

Weinberg, B., Talbot, M., \& Whitehead, M. (2013). Feature: "Physical Literacy."

ICSSPE Bull J Sport Sci Phys Educ, 65, 296.

Welk, G. J. (1999). The Youth Physical Activity Promotion Model: A Conceptual

Bridge Between Theory and Practice. Quest, 51, 5-23.

https://doi.org/10.1080/00336297.1999.10484297

Welsh Government. (n.d.). Welsh Index of Multiple Deprivation (WIMD).

Retrieved September 18, 2018, from https://gov.wales/statistics-andresearch/welsh-index-multiple-deprivation/?lang=en

Welsh Government. (2012). The Children and Families (Wales) Measure 2010

(Commencement No. 5) Order 2012. Retrieved May 23, 2016, from

http:/www.legislation.gov.uk/wsi/2012/2453/contents/made

Welsh Government. (2015a). Health Behaviour in School-aged Children Wales

Survey 2013/14. Retrieved from http://gov.wales/statistics-and-research/healthbehaviour-school-aged-children/?lang=en

Welsh Government. (2015b). National Survey for Wales 2014/15. Retrieved from http://gov.wales/statistics-and-research/national-survey/?tab=current\&lang=en

Welsh Government. (2015c). Welsh Health Survey 2014. Retrieved from

http:/gov.wales/statistics-and-research/welsh-health-survey/?lang=en

Welsh Government. (2017). National Survey for Wales 2016/17. Retrieved

September 18, 2018, from https://gov.wales/statistics-and-research/nationalsurvey $/$ tab $=$ current\&lang $=$ en

Whitehead, M. (2010). Physical Literacy: throughout the lifecourse. London: Routledge.

Wiart, L., \& Darrah, J. (2001). Review of four tests of gross motor development. Developmental Medicine and Child Neurology, 43, 279-285.

Wickham, S., Anwar, E., Barr, B., Law, C., \& Taylor-Robinson, D. (2016). Poverty and child health in the UK: using evidence for action. Archives of Disease in Childhood, 101(8), 759-766. https://doi.org/10.1136/archdischild-2014306746

Wind, A. E., Takken, T., Helders, P. J. M., \& Engelbert, R. H. H. (2010). Is grip strength a predictor for total muscle strength in healthy children, adolescents, 
and young adults? European Journal of Pediatrics, 169(3), 281-287.

https://doi.org/10.1007/s00431-009-1010-4

Wolin, K. Y., Yan, Y., \& Colditz, G. A. (2011). Physical activity and risk of colon adenoma: A meta-analysis. British Journal of Cancer, 104(5), 882-885. https://doi.org/10.1038/sj.bjc.6606045

World Health Organization. (n.d.). Health Impact Assessment (HIA): Glossary of terms used. Retrieved September 1, 2018, from http://www.who.int/hia/about/glos/en/index1.html

World Health Organization. (2015). Obesity and overweight. Retrieved July 31, 2015, from http://www.who.int/en/news-room/fact-sheets/detail/obesity-andoverweight

World Health Organization. (2018a). Global action plan on physical activity 2018 2030: more active people for a healthier world.

World Health Organization. (2018b). Physical activity. Retrieved from http://www.who.int/ncds/prevention/physical-activity/introduction/en/

Yoon, B., Kim, C., Lim, H., Kim, Y., Im, J., Paik, I., ... Suh, S.-H. (2009). Association of physical performance and health-related factors among elderly Korean subjects. International SportMed Journal, 10(4), 205-215.

Zhu, W., \& Cole, E. L. (1996). Many-faceted Rasch calibration of a gross motor instrument. Research Quarterly for Exercise and Sport, 67(1), 24-34. 


\section{Appendices}

\section{Appendix I: Surveillance Surveys on Physical Activity and Seden- tary Behaviour of Children in Wales (Study 1)}

The annual Welsh Health Survey (Welsh Government, 2015c), provides information about the health and health-related lifestyles of people living in Wales. It covers a range of health-related topics, including health status, lifestyle and health behaviours (including physical activity), and health service use. The survey data is collected through a combination of methods. Namely, household data is collected in face-toface interviews, whereas individual data is collected using paper questionnaires. Each adult $(16+$ years $)$ in the household was given a questionnaire to complete. Two questionnaires were designed for parents to complete on behalf of children aged 0 to 3 , and children aged 4 to 12 ; a third questionnaire was given to children aged 13 to 15 to complete on their own behalf.

http:/gov.wales/statistics-and-research/welsh-health-survey/?lang=en

The Health Behaviour in School Children (HBSC) survey (every 4 years - most recent 2013/14) (Welsh Government, 2015a), collected data on 9,055 secondary school children (aged 11-16 in school years 7 to 11 ) in Wales. The survey was carried out through interviewer administered paper self-completion sessions in class. The survey focuses on and provides data on key indicators of health or health behaviour in young people in Wales under the headings: social context of health; health outcomes; health behaviours; and risk behaviours.

http://gov.wales/statistics-and-research/health-behaviour-school-aged-

children/?lang=en

The School Sport Survey conducted by Sport Wales (Sport Wales, 2015c), has the greatest reach of all surveys completed in Wales. In the 2015 survey, 115,398 Year 3-11 pupils in 1,000 schools took part in the survey, and 1,094 teachers completed a School Sport Survey provision questionnaire. The survey monitors and tracks trends in sports participation - covering both community and school participation and tracks 
the provision of PE and school sport, as well as teachers' and pupils' attitudes to PE and sport.

http://sportwales.org.uk/research--policy/surveys-and-statistics/school-sportsurvey.aspx

The Further Education Survey is also conducted by Sport Wales (Sport Wales, $2015 b$ ). This survey was the first survey of students in further education colleges in Wales. In 2015, 4,568 students (aged 16 to 20+ years) completed the online questionnaire, obtaining data on core measures of well-being and frequency of sport participation. This survey is like the School Sport Survey, collecting data on participation in sport and physical activity, volunteering, and attitudes to sport. This allows the School Sport Survey and Further Education Survey to be combined and data to cover children and young people from 7 through to $20+$ years. http://sport.wales/research--policy/surveys-and-statistics/statistics.aspx

The National Survey for Wales (Welsh Government, 2015b), is a face-to-face survey of people across Wales. Each year over 14,000 people aged 16 and over were asked for their opinions on a wide range of issues affecting them and their local area. Interviewers randomly selected one adult (aged 16+) in each household, and carried out a 25-minute face-to-face interview. In the most recent 2014/15 survey, a total of 14,285 interviews were achieved. Proxy data on health and related areas are available which includes data on the environment, active travel and health. http://gov.wales/statistics-and-research/national-survey/?lang=en 


\section{The Welsh Health Survey 2015}

One question asks how health condition(s) affects individual carrying out functional physical activities and ADL

\section{Walking (over past 7 days)}

Across how many days did you walk for 10 mins at a time?

How much time did you spend walking on one of these days?

Describe walking pace?

Was the effort used for 10 minutes walking enough to make you sweat, increase HR etc.?

\section{Moderate physical activity (over past 7 days)}

How many days last week did you engage in moderate physical activity lasting at least 10 mins?

How much time on average did you spend doing moderate activity on one of those days?

\section{Vigorous physical activity (over past 7 days)}

How many days last week did you engage in vigorous physical activity lasting at least 10 mins?

How much time on average did you spend doing vigorous physical activity on one of those days?

\section{Sitting time (over past 7 days)}

How much time over the last 7 days did you spend sitting on an average week day?

How much time over the last 7 days did you spend sitting on an average weekend day?

\section{Health Behaviour in School-aged Children in Wales 2013-2014}

1. Over the past 7 days, on how many of the days were you physically active for 60 minutes or more?

2. What is the main transport mode to and from school? (active transport).

3. Out of school, how many hours do you spend doing physical activity that makes you out of breath or sweat? 


\section{School Sport Survey 2015 (similar questions for Further Education Survev 2015)}

\section{SPORTS}

This section is about sport you do when you are not in PE lessons

1. Which of these sports have you done since you have been in [year]?

2. Which of these sports have you done since you have been in [year]?

\section{FOR ALL SPORTS TICKED ABOVE}

a. Have you played in a school club (at lunchtime, after school, or at breakfast club), since you have been in Year X?

b. Have you played in a club not at school since you have been in Year X?

c. Have you played basketball anywhere else since you have been in Year $\mathrm{X}$ ? This could be at home, or with your friends, or in the park or at a leisure centre for example.

For pupils in Years 5-6 ONLY. Years 3 and 4 do not get asked questions about frequency of participation in individual sports

\section{School clubs:}

1. About how often did you play (sport) in a school club, since you have been in Year X? (at a lunchtime club, after school club or at breakfast club)

\section{Clubs not at school:}

1. About how often did you play (sport) in a club not at school, since you have been in Year X?

\section{Overall Participation in Sport:}

1. Overall, since you have been in [Q2], about how often have you taken part in sport in a school club? (at lunchtime, after school, or at breakfast club)

2. Overall, since you have been in [Q2], about how often have you taken part in sport in a club not at school? This could be a sports club, or a community club or a leisure centre club for example

\section{Competitive sport:}

1. Since you have been in $\{x\}$, have you represented your school in a sports match or competition against another school? 


\section{Sports clubs:}

1. Are you a member of a sports club?

\section{Places you do sport:}

1. Have you used any of these for sport or exercise, since you have been in [Q2a]? Please tick all that apply.

\section{Leisure centres (If yes to leisure centre above)}

- Since you have been in [x], how often did you visit a sports or leisure centre to do an activity outside of school time?

\section{Sports you would like to do:}

1. Which sports, if any, would you like to do more of? Please tick all that apply.

- I would do more sport if... Please tick all that apply.

\section{Family and friends:}

1. Do your family or friends take part in sport? Please tick all that apply.

2. Who do you take part in sport with? Please tick all that apply.

\section{Well-being:}

1. How much do you think PE lessons and sport help you to have a healthy lifestyle?

- How often do you feel comfortable taking part in PE lessons and school sport?

- How confident are you in trying new activities?

- How often do you feel that pupils' ideas about PE and school sport are listened to?

- How much do you enjoy PE lessons?

- How much do you enjoy doing sport at after-school or lunchtime clubs?

- How much do you enjoy doing sport when you are not at school? This could be at a club or a leisure centre for example

\section{Leisure time:}

1. Which of these do you do when not in school?

\section{ALL ANSWERS SELECTED IN Q31 LISTED AS ANSWER OPTIONS BELOW}

2. In a normal week, which three of these things do you spend the most time doing, when you are not at school? Please tick up to three activities. 
3. Why do you do (sport)? Please tick all that apply.

4. Why do you do (sport)? Please write in the box below.

\section{Travel to school:}

1. On a normal day, how do you get to school?

- Walk

- $\quad$ By car

- $\quad$ By taxi

- $\quad$ By bus

- Cycle

- Scooter or skate

- Other

2. On a normal day, how long does it usually take to travel from your home to school?

- Less than 5 minutes

- 6 to 9 minutes

- 10 to 15 minutes

- 16 to 19 minutes

- 20 to 24 minutes

- 25 to 29 minutes

- 30 minutes or more

\section{$\underline{\text { National Survey of Wales } 2014 \text { - } 2015}$}

1. Active travel (children) - how do they get to primary/secondary school, how long is the journey?

2. Active travel - only focuses on walking or cycling to and from work, shops or to visit friends 
Appendix II: Swan-Linx Standard Operating Procedure and Training Tool

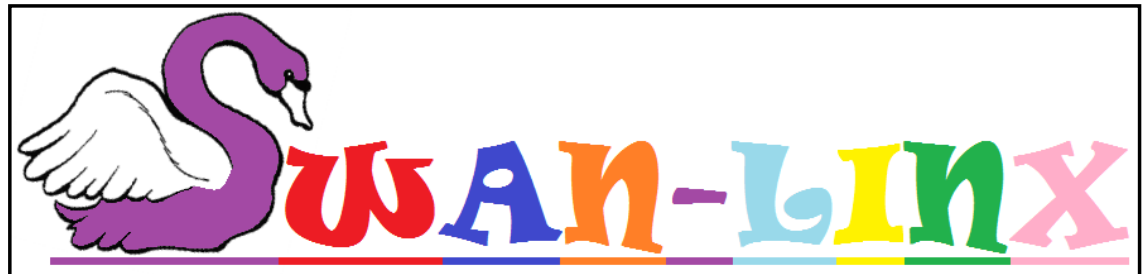

Field Measures of Health and Fitness in Children

\section{Standard Operating Procedure and Training Tool}

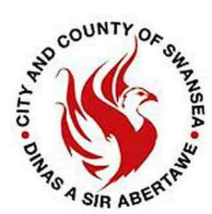




\section{Field Fitness Test Batteries}

There have been many methods to assess the different components of fitness in children over the past few decades. Numerous studies have used a battery of field fitness tests to assess fitness in children in a relatively non-invasive, cost effective and simple manner (Ekblom et al., 2005). This has allowed fitness testing to evolve from focusing on sporting performance to assessing health-related outcomes (Freedson et al., 2000). There are many fitness testing batteries that have been used, including; AAHPER (American Alliance for Health and Physical Education and Recreation), Youth Fitness Test, YMCA Fitness Test and FITNESSGRAM (Freedson et al., 2000) in the US. In Europe, possibly the most widely used fitness test battery is EUROFIT (Adam, Klissouras, Ravazollo, Renson \& Tuzworth, 1988). EUROFIT was developed by the council of Europe and provides data on several components of skill and health related fitness. It includes simple measures of CRF, agility, hand-eye coordination, BMI, upper body strength, muscular endurance and balance.

\section{SwanLinx "Fitness Fun Day": Field Based Motor Fitness Assessment Methods}

Testing methods used during the Fitness Fun Day are based on the same methods used by SportsLinx in Liverpool since 1998. The primary schools are invited to participate in the project and assigned a date and time to attend the Indoor Training Centre (ITC) at Swansea University. All children had the option to withdraw from any part of the study at any time. Data collection started in 2012 and this year 3000 children will have taken part. Ethics and the protection of data are paramount to us and Swanlinx adheres to the principles set out in the Helsinki Declaration of 1972. To this end Head teachers gave written informed consent, parents gave passive consent and children written informed assent. The study was approved by institutional research ethics committee (ref number PG14/2014/007). 


\section{Standard Operating Procedure for SwanLinx Fitness Fun Days}

\section{Prior to the fitness fun day:}

1) Initial contact with the school (2-3 months before proposed testing period)

- Arrange to meet PE coordinator or head teacher at the school.

- Preparation:

$>$ Print off a copy of the feedback template, copies of the information sheets and consent forms, and reports on fitness, obesity and lifestyle to provide to teachers.

- Meeting Objectives:

$>$ Convince school to take part in the project. (Do this by explaining what is involved in the project, the aims of the project and what the school will gain from taking part)

- What needs to be explained during the meeting:

$>$ The project aims to determine the health, fitness, diet and lifestyle of children aged 9-11 in Swansea.

$>$ The project has been running in Liverpool since 1996 where over 80000 children have taken part. The project is now being started in Swansea.

$>$ The project involves a fitness fun day at Swansea University's Indoor Training Centre (ITC) and an online questionnaire.

$>$ The fitness fun day takes half a day (approximately two and a half hours). It takes place in a morning or afternoon session (9.30-12 or 12.30-3).

$>$ The project is free to take part in; we only ask that you cover the costs for your transport to and from the ITC.

$>$ The fitness fun day involves the children doing a sit \& reach test, handgrip strength test, $10 \times 5 \mathrm{~m}$ shuttle run, standing broad jump, speed bounce and the $20 \mathrm{~m}$ Multistage Shuttle Run (bleep test). The children also have their weight, height and sitting height taken. The children are all given numbers and the data is all stored securely. Only the anonymous data will be used.

> The children will then complete an online questionnaire called CHAT (Child's Health and Activity Tool). It asks questions about their lifestyle, diet, physical activity and mental wellbeing.

$>$ The school will be given access to the anonymous data for curriculum and educational use. The City \& County of Swansea will also the deliver a taster session on the sports that the children would most like to try, which is something gathered from the questionnaire.

- What needs to be found out during the meeting:

$>$ Find out if the school would like to take part.

$>$ Find out the ICT facilities at the school.

$>$ Find out the number of children in Years 5 and 6. 
Find out if there are any dates that the school definitely can't attend a fitness fun day.

Ask to be provided with copy of the school registers.

2) Find out dates that the City \& County of Swansea are unable to attend.

3) Find potential dates from ITC for fitness fun day ( 2 months prior)

- Find out what potential dates are available:

$>$ Indoor Training Centre contact number - 01792602400

$>$ Speak to Andrew Griffiths (manager) at the Indoor Training Centre

$>$ Morning session: 8.30-12

$>$ Afternoon session: $12-3.30$

$>$ All day ( 2 schools): $8.30-3.30$

- State that you need access to all of the ITC.

- State the equipment that you will need access to:

$>$ Speed bounce foam wedges and mats

$>$ Standing long jump mat and board

$>$ Marking cones

4) Propose the potential dates to the schools, confirm at the ITC.

5) Reserve equipment from Swansea University Sports Science Physiology Laboratory

- Complete a Lab and Equipment Requirement form and return to Wendy Clark or Rebecca Dietzig (w.a.clark@swansea.ac.uk;

r.e.dietzig@swansea.ac.uk)

$>$ Confirm booking, contact number - 01792602028

- Equipment needed:

$>$ Sit \& Reach box

$>$ Handgrip Dynanometer (x2)

$>$ Weighing Scales

$>$ Height Stadiometer

$>$ Sitting Height Stadiometer

$>$ Clip boards (x9)

$>30 \mathrm{~m}$ Measuring Tape

$>$ Stopwatches $(x 6)$

$>$ Sound system (confirm with AYP team)

- In addition:

$>$ Numbers (labels)

$>$ Testing Sheets

$>$ Extra consent forms

$>$ Copy of testing protocols

$>$ Introduction Speech

$>$ Folders for Consent forms

$>$ SOPs (Fitness fun day \& CHAT)

$>$ Confirm number of helpers (should be at least between 11-15 people preferably between 14-19)

$>$ Confirm with school about arrival time and venue 


\section{6) Arrange for help Young Ambassadors}

- Contact Gavin Lewis to arrange for help on the fitness fun day from Young Ambassadors (8-10)

7) Reserve PA system from City \& County of Swansea and ensure copy of the 20m Multistage Shuttle Run CD works.

8) Print off copies of the head teacher, parent and children information sheets and consent/assent forms.

- Include several extras of each to provide to school.

9) Make copies of recording sheets and test protocols for each of the tests. 10) Write numbers on sticky labels to give to the children.

\section{During the fitness fun day:}

- Introductory Speech:

"Thank you for coming down to the university today.

First of all I need to go over the safety for this morning.

In the case of a fire, the doors are clearly marked and you will remain with your groups and leave your bags and follow us outside to the assembly point.

If you need the toilet at any point then please let one of the research team or YAs or your teacher know, the same if you need to get a drink.

Also if you begin to feel ill or injure yourself let one of us know.

I'll tell you a little bit about the study before we get started.

The study is looking at the health and fitness of 9-11 year old children in Swansea. You guys are one of the many schools to take part in the study this year, which is really exciting, and eventually every primary school will be tested in Swansea.

You guys have come down here today to do a series of fitness activities. Some of you may have done some of them before, and some of you won't have. These include $10 \times 5 \mathrm{~m}$ sprint, sit and reach, handgrip strength, speed bounce, standing long jump and the bleep test.

You will also have your weight, height and sitting height measured.

In a moment you will all be given a number. This is the number that you give to the researchers or YAs at each station. So you don't have to worry about giving your name. All of the data is kept private so we can't look at somebody's score and say "oh Charlie got 50 in the bleep test" it will simply have his number.

If there is anything you don't want to do then just say and you don't have to do it, we won't pressure you or anything. Also you don't need to tell your friends how you did in each activity; it's not a competition between each other we just want you to do the best you can do.

Now if I'm going to split you into 3 groups." 


\section{Anthropometric measures}

\section{Explaining the anthropometric measures to children:}

\begin{tabular}{|c|c|c|c|}
\hline & \multicolumn{3}{|c|}{ Assessment item } \\
\hline & Height & Weight & Sitting height \\
\hline What will you do? & $\begin{array}{l}\text { You will have to } \\
\text { stand as straight as } \\
\text { possible with your } \\
\text { shoes off then } \\
\text { breath in to get your } \\
\text { best height }\end{array}$ & $\begin{array}{l}\text { Stand on the scales } \\
\text { without shoes and } \\
\text { wearing a t-shirt and } \\
\text { shorts/gym wear }\end{array}$ & $\begin{array}{l}\text { Sit on the special } \\
\text { table and have your } \\
\text { height measured } \\
\text { from the bottom of } \\
\text { your bottom to the } \\
\text { top of your head }\end{array}$ \\
\hline Why? & $\begin{array}{l}\text { We want to record } \\
\text { how much you are } \\
\text { growing and compare } \\
\text { some of your other } \\
\text { results to your height }\end{array}$ & $\begin{array}{l}\text { We want to record } \\
\text { how much you are } \\
\text { growing and compare } \\
\text { some of your other } \\
\text { results to your weight }\end{array}$ & $\begin{array}{l}\text { We can use this to } \\
\text { work out how long } \\
\text { your kegs are and } \\
\text { how you are } \\
\text { maturing physically }\end{array}$ \\
\hline What will it tell you? & $\begin{array}{l}\text { This will tell you how } \\
\text { tall you are in cms } \\
\text { and metres. If you } \\
\text { measure your height } \\
\text { every } 3 \text { months it will } \\
\text { let you know how } \\
\text { fast you are growing }\end{array}$ & How heavy you are. & $\begin{array}{l}\text { How long your legs } \\
\text { are. } \\
\text { How long your upper } \\
\text { body is. }\end{array}$ \\
\hline Is it competitive? & $\begin{array}{l}\text { Not at all, it is your } \\
\text { measure and you } \\
\text { can't change it }\end{array}$ & $\begin{array}{l}\text { No not at all. But you } \\
\text { can have a healthy } \\
\text { weight by eating } \\
\text { healthy food and } \\
\text { being active for at } \\
\text { least } 60 \text { mins per day }\end{array}$ & $\begin{array}{l}\text { Not at all, it is your } \\
\text { measure and you } \\
\text { can't change it }\end{array}$ \\
\hline $\begin{array}{l}\text { How will you feel } \\
\text { after it? }\end{array}$ & $\begin{array}{l}\text { Hopefully you will } \\
\text { feel good knowing } \\
\text { how tall you are }\end{array}$ & $\begin{array}{l}\text { Some people are } \\
\text { worried about their } \\
\text { weight and what they } \\
\text { weigh. Nobody } \\
\text { should be scared } \\
\text { about their weight }\end{array}$ & $\begin{array}{l}\text { Hopefully you will } \\
\text { feel good knowing } \\
\text { how your height is } \\
\text { split between the } \\
\text { length of your legs } \\
\text { and upper body }\end{array}$ \\
\hline $\begin{array}{c}\text { How can you help } \\
\text { others? }\end{array}$ & $\begin{array}{l}\text { Make sure that you } \\
\text { help other people by } \\
\text { respecting them so } \\
\text { they can keep their } \\
\text { results to themselves }\end{array}$ & $\begin{array}{l}\text { Make sure that you } \\
\text { help other people by } \\
\text { respecting them so } \\
\text { they can keep their } \\
\text { results to themselves }\end{array}$ & $\begin{array}{l}\text { Make sure that you } \\
\text { help other people do } \\
\text { the test and keep } \\
\text { their results to } \\
\text { themselves }\end{array}$ \\
\hline Hints for instructors & $\begin{array}{l}\text { Make sure that } \\
\text { children know that } \\
\text { they can't change } \\
\text { their height. Tell } \\
\text { them to breath in, } \\
\text { keep their head level } \\
\text { and make themselves } \\
\text { as long as possible }\end{array}$ & $\begin{array}{l}\text { It is really important } \\
\text { to make sure that } \\
\text { you reinforce you are } \\
\text { what you weigh, this } \\
\text { is your weight. }\end{array}$ & $\begin{array}{l}\text { Make sure that } \\
\text { children know that } \\
\text { they can't change } \\
\text { their height. Tell } \\
\text { them to breath in } \\
\text { and make themselves } \\
\text { as long as possible }\end{array}$ \\
\hline
\end{tabular}




\section{0m Multistage Fitness Test (Shuttle Run)}

The $20 \mathrm{~m}$ multistage fitness test (MSFT) is a commonly used maximal running aerobic fitness test. It is also known as the 20 meter shuttle run test, beep or bleep test.

\section{Equipment:}

- $20 m$ Measuring tape

- Marking cones

- $\quad$ Beep test CD

- CD player

- Recording sheets

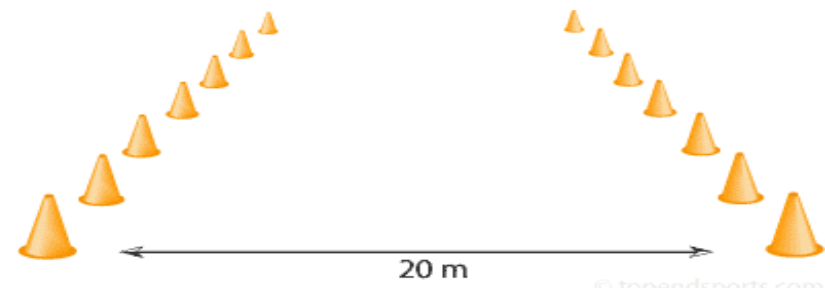

- Flat non-slip surface

\section{Personnel:}

If there are two bleep tests running side by side need minimum of 2 people for each test.

- $\quad 5$ people

\section{Procedure:}

This test involves continuous running between two lines $20 \mathrm{~m}$ apart in time to recorded beeps. The subjects stand behind one of the lines facing the second line, and begin running when instructed by the $C D$ recording. The speed at the start is quite slow. The subject continues running between the two lines, turning when signalled by the recorded beeps. After about one minute, a sound indicates an increase in speed, and the beeps will be closer together. This continues each minute (level). If the line is reached before the beep sounds, the subject must wait until the beep sounds before continuing. If the line is not reached before the beep sounds, the subject is given a warning and must continue to run to the line, then turn and try to catch up with the pace within the next 'beep'. The test is stopped if the subject fails to reach the line (within 2 meters) for two consecutive beeps.

\section{Scoring:}

The athlete's score is the number of shuttles $(20 \mathrm{~m})$ reached before they were unable to keep up with the recording. Record the last lap completed (not necessarily the lap stopped at). 


\section{Explaining the 20m MSFT to children:}

\begin{tabular}{|c|c|}
\hline & Assessment item \\
\hline & $20 \mathrm{~m}$ shuttle run (bleep test) \\
\hline What will you do? & $\begin{array}{c}\text { You will be asked to run back and forth over a } 20 \text { metre } \\
\text { distance in time with a beep }\end{array}$ \\
\hline Why? & $\begin{array}{c}\text { We want to know how your fitness level is. This type of fitness } \\
\text { puts more demands on your heart lungs and legs and your } \\
\text { ability to keep going. We know that this is related to your } \\
\text { health }\end{array}$ \\
\hline What will it tell you? & $\begin{array}{l}\text { You will run as far as you can and you will know how many } 20 \\
\text { metre shuttles (runs) that you completed. If you do } 20 \text { shuttles } \\
\text { that means you have completed } 400 \mathrm{~m} \\
\text { We know that a healthy fitness score is } 25 \text { shuttles for girls and } \\
33 \text { for boys }\end{array}$ \\
\hline Is it competitive? & $\begin{array}{l}\text { The most important thing about this task is that you do your } \\
\text { best and work as hard as you can. We know how hard you are } \\
\text { working. } \\
\text { IT IS NOT A RACE }\end{array}$ \\
\hline $\begin{array}{l}\text { How will you feel after } \\
\text { it? }\end{array}$ & $\begin{array}{c}\text { You should feel very tired initially and your heart rate and } \\
\text { breathing will be much faster BUT you will recover very quickly. } \\
\text { IF you have done your best you will feel a sense of achievement } \\
\text { for you. }\end{array}$ \\
\hline $\begin{array}{l}\text { How can you help } \\
\text { others? }\end{array}$ & $\begin{array}{l}\text { When you have finished you will get a break to recover. This } \\
\text { will happened quickly. Before you cool right down we aim to } \\
\text { keep everybody involved by joining all those who are left } \\
\text { running. You may want to join in next to a friend to help them } \\
\text { do their best. In. You could even run with a friend to help } \\
\text { motivate them }\end{array}$ \\
\hline Hints for instructors & $\begin{array}{l}\text { This test has been criticized for not recording accurate results. } \\
\text { This is due to low motivation levels of children and not setting } \\
\text { the test up in a +ve cooperative atmosphere. When children } \\
\text { stop and get their breath back they all need to go back in and } \\
\text { help out a mate. At the very end the person with the best score } \\
\text { should not be running alone }\end{array}$ \\
\hline
\end{tabular}




\section{Speed Bounce}

Speed Bounce events are part of the Sportshall series designed for the indoor use of school age competitors. This event is an exciting test of an athletes speed, rhythm and coordination, involving double footed jumps over a foam wedge.

\section{Equipment:}

- Stopwatch

- Foam wedge

- Recording sheets

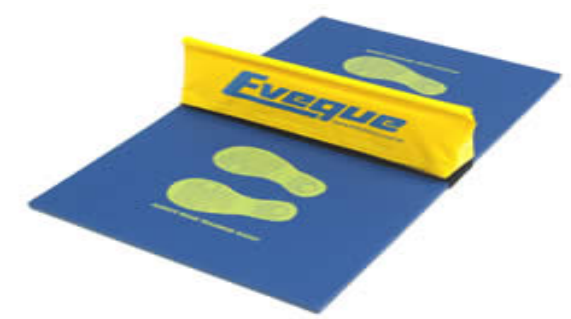

\section{Personnel:}

- 3 people

\section{Procedure:}

Participants must wear suitable footwear. Not to be completed in bare feet.

- Speed Bounce is a two-footed jump in which the participant must take off and land on both feet. - The participant's feet should leave the mat simultaneously and land on the mat simultaneously.

- The athlete should cross the wedge as many times as possible within 30 seconds.

- If an incorrect technique is used, the participant is stopped, offered an explanation as to the correct technique, and then allowed a fresh trial after an adequate rest period.

- An official should call out when 10 seconds are remaining.

- The number of correct bounces is counted. It is not an offence to clip or brush the wedge.

- A practice trial is allowed - about 5 to 10 bounces provide an ideal opportunity to spot potential problems with technique.

- Two trials are allowed.

\section{Scoring:}

Two officials should count the "correct" bounces. They should then liaise and agree on the number completed within the 30 second period. 


\section{Explaining the speed bounce to children:}

\begin{tabular}{|c|c|}
\hline & Assessment item \\
\hline & Speed Bounce \\
\hline What will you do? & 2 footed jump back and forth as many times in 30 seconds \\
\hline Why? & $\begin{array}{l}\text { This tells us how well coordinated you are and how you can } \\
\text { keep this going when you're tired }\end{array}$ \\
\hline What will it tell you? & $\begin{array}{l}\text { How fit you are when doing something that requires you to } \\
\text { jump quickly in along burst }\end{array}$ \\
\hline Is it competitive? & $\begin{array}{l}\text { Yes it is. Motivate yourself to keep going when you're tired. Tell } \\
\text { yourself to do your best }\end{array}$ \\
\hline $\begin{array}{l}\text { How will you feel after } \\
\text { it? }\end{array}$ & $\begin{array}{l}\text { You should feel very warm and tired in your legs. If you don't } \\
\text { you will not have done your best! }\end{array}$ \\
\hline $\begin{array}{l}\text { How can you help } \\
\text { others? }\end{array}$ & Help motivate them if they want you to. \\
\hline Hints for instructors & $\begin{array}{l}\text { This is definitely related to sport and dance performance and } \\
\text { relates to how well coordinated a child is. It is important to ask } \\
\text { children to coordinate their arms and legs and keep their head } \\
\text { as central over the barrier as possible. Motivate them by using } \\
\text { the group to shout them on but only if the child wants them to. }\end{array}$ \\
\hline
\end{tabular}




\section{Sit \& Reach}

The sit and reach test is a common measure of flexibility, and specifically measures the flexibility of the lower back and hamstring muscles. This test is important as tightness in this area is implicated in lumbar lordosis, forward pelvic tilt and lower back pain.

The basic outline of the sit and reach test is described below.

\section{Equipment:}

- $\quad$ Sit \& Reach box

- Recording sheets

\section{Personnel:}

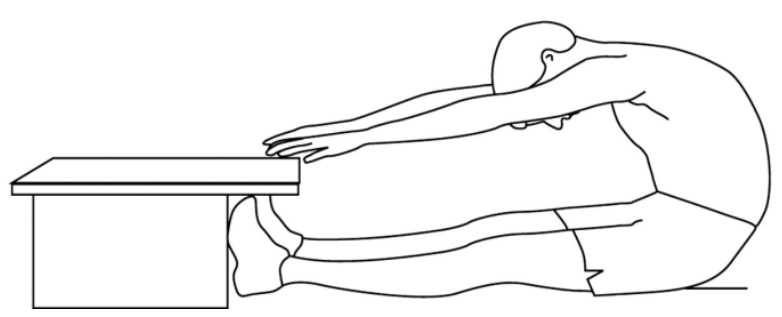

- $\quad$ 1-2 people

\section{Procedure:}

This test involves sitting on the floor with legs stretched out straight ahead. Shoes should be removed. The soles of the feet are placed flat against the box, shoulderwidth apart. Both knees should be locked and pressed flat to the floor - the tester may assist by holding them down. With the palms facing downwards, and the hands on top of each other or side by side, the participant reaches forward along the measuring line as far as possible. Ensure that the hands remain at the same level, not one reaching further forward than the other. After some practice reaches, the subject reaches out and holds that position for one-two seconds while the distance is recorded. Make sure there are no jerky movements.

\section{Scoring:}

The score is recorded to the nearest centimetre as the distance reached by the hand. 


\section{Explaining the sit and reach to children:}

\begin{tabular}{|c|c|}
\hline & Assessment item \\
\hline & Sit and Reach \\
\hline What will you do? & $\begin{array}{l}\text { This tells you how far you can stretch and how flexible and } \\
\text { bendy you are }\end{array}$ \\
\hline Why? & $\begin{array}{c}\text { Muscles that stretch well are healthier. As you get older you } \\
\text { will be less likely to have back pain or get injured playing sport } \\
\text { if you have good flexibility }\end{array}$ \\
\hline What will it tell you? & $\begin{array}{l}\text { This tells you if you can touch your toes. You will have to stretch } \\
\text { (make longer) the muscles at the back of the legs and in the } \\
\text { lower part of your back }\end{array}$ \\
\hline Is it competitive? & $\begin{array}{l}\text { No, this is not competitive. In fact you should stretch forward } \\
\text { slowly. You should make sure that you are warm when you do } \\
\text { it. }\end{array}$ \\
\hline $\begin{array}{l}\text { How will you feel after } \\
\text { it? }\end{array}$ & $\begin{array}{l}\text { You might feel a little discomfort on the back of the legs but this } \\
\text { is because you are making the muscles in the legs and backs } \\
\text { stretch as far as they can safely go. }\end{array}$ \\
\hline $\begin{array}{l}\text { How can you help } \\
\text { others? }\end{array}$ & $\begin{array}{l}\text { By following the proper instructions and stretching as far as you } \\
\text { can. }\end{array}$ \\
\hline Hints for instructors & $\begin{array}{l}\text { Make sure that you talk to children that muscles become more } \\
\text { stretchable when they are warm. Also mention some activities } \\
\text { that require a lot of flexibility, dance, gymnastics, swimming, } \\
\text { but that all activities need balanced flexibility to protect joints. }\end{array}$ \\
\hline
\end{tabular}




\section{Handgrip Dynanometer Strength Test}

The purpose of the handgrip strength test is to measure the maximum isometric strength of the hand and forearm muscles. Handgrip strength is important for any sport in which the hands are used for catching, throwing or lifting. Also, as a general rule people with strong hands tend to be strong elsewhere, so this test is often used as a general test of strength.

\section{Equipment:}

- Handgrip Dynamometer

- Recording sheets

\section{Personnel:}

- $\quad$ 1-2 people

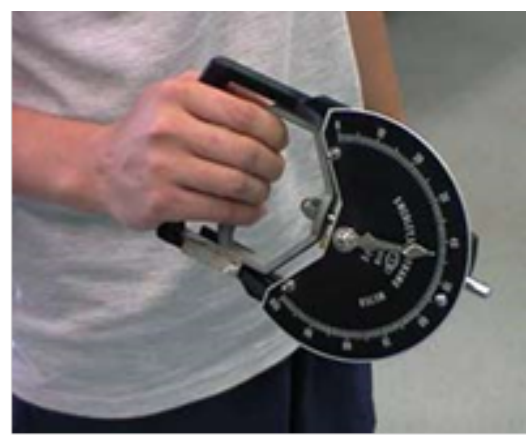

\section{Procedure:}

- The handle of the dynamometer should be adjusted if required - the base should rest on first metacarpal (heel of palm), while the handle should rest on middle of four fingers.

- The participant holds the dynamometer in the hand to be tested with the arm held (extended) above their head

- When ready the subject squeezes the dynamometer with maximum effort (maintained for 3-5 seconds), they bring down their arm to their side in a smooth motion. $\rightarrow$ The extended arm being swung from above the head to by the side during the squeezing motion.

- No other body movement is allowed.

- Make sure both hands are measured

\section{Scoring:}

Record the reading for each hand 


\section{Explaining the handgrip to children:}

\begin{tabular}{|c|c|}
\hline & Assessment item \\
\hline & "Handgrip \\
\hline What will you do? & $\begin{array}{l}\text { You will adjust instrument to suit your hand size and then grip it } \\
\text { as tight as you can. }\end{array}$ \\
\hline Why? & This a measure of your basic strength \\
\hline What will it tell you? & $\begin{array}{l}\text { It tells you how hard you can grip things and how strong you } \\
\text { are }\end{array}$ \\
\hline Is it competitive? & $\begin{array}{l}\text { Yes you can be competitive against yourself, try as hard as you } \\
\text { can, even shout aaaaah when you do it if you want....it could } \\
\text { improve your score. It's also good to compete your right hand } \\
\text { against your left...... which one is stronger? }\end{array}$ \\
\hline $\begin{array}{l}\text { How will you feel after } \\
\text { it? }\end{array}$ & $\begin{array}{l}\text { You will feel that you have exercised the muscles in your lower } \\
\text { arms }\end{array}$ \\
\hline $\begin{array}{l}\text { How can you help } \\
\text { others? }\end{array}$ & $\begin{array}{l}\text { Help motivate them if they want you to. Say c'mon squeeeeze!! } \\
\text { Go for it and so on! }\end{array}$ \\
\hline Hints for instructors & $\begin{array}{l}\text { Each kg recorded is about the same as a bag of sugar. Tell the } \\
\text { children how bags of sugar they can lift with their fingertips. Or } \\
\text { let them compare left and right side......Who can get an exact } \\
\text { match? } \\
\text { Also what percentage of their body weight could they lift? } \\
\text { So if the get } 15 \text { on handgrip and they are } 30 \text { kgs in weight they } \\
\text { can lift half their bodyweight with their fingertips.....amazing!! }\end{array}$ \\
\hline
\end{tabular}




\section{$10 \times 5 m$ Shuttle Run Test}

The $10 \times 5 \mathrm{~m}$ shuttle run test is a test of speed and agility.

\section{Equipment:}

- A flat non-slip surface

- Stopwatch

- Measuring Tape

- Marker cones

- Recording sheets

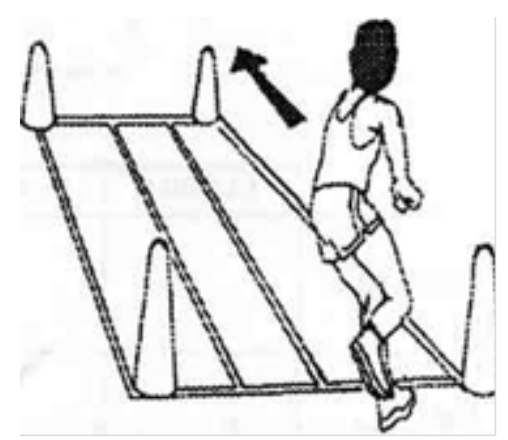

\section{Personnel:}

- $\quad$ 2-3 people

\section{Procedure}

- Marker cones and/or lines are placed five meters apart.

- Start with a foot at one marker.

- When instructed by the timer, the participant runs to the opposite marker, turns and returns to the starting line.

- This is repeated five times without stopping (covering 50 meters total).

- At each marker both feet must fully cross the line.

\section{Scoring}

Record the total time taken to complete the $50 \mathrm{~m}$ course.

Record to two decimal places $\rightarrow$ e.g. 17.35 


\section{Explaining the $10 \times 5 \mathrm{~m}$ Shuttle Run to children:}

\begin{tabular}{|c|c|}
\hline & Assessment item \\
\hline & $10 \times 5 m$ Shuttle Run \\
\hline What will you do? & You will sprint back and forth over a $5 \mathrm{~m}$ distance 10 times \\
\hline Why? & $\begin{array}{l}\text { This tells us how agile you are and how quickly you can change } \\
\text { direction when running at speed }\end{array}$ \\
\hline What will it tell you? & $\begin{array}{l}\text { Whether you are fast AND agile and how you may use this in } \\
\text { sport or dance }\end{array}$ \\
\hline Is it competitive? & $\begin{array}{l}\text { Yes compete against yourself and try to turn as quickly as } \\
\text { possible to achieve your best time }\end{array}$ \\
\hline $\begin{array}{l}\text { How will you feel after } \\
\text { it? }\end{array}$ & $\begin{array}{l}\text { You should feel a little tired but also great after running so fast- } \\
\qquad \text { as fast as you can }\end{array}$ \\
\hline $\begin{array}{l}\text { How can you help } \\
\text { others? }\end{array}$ & $\begin{array}{l}\text { Help motivate them if they want you to. } \\
\text { You could even run with a friend to help motivate them }\end{array}$ \\
\hline Hints for instructors & $\begin{array}{l}\text { This is a measure of speed and agility and crucial for successful } \\
\text { performance in sport and dance. } \\
\text { Key is to show children how to turn quickly by turning the head } \\
\text { to look back in the direction they want to go when they get to } \\
\text { the line. }\end{array}$ \\
\hline & in children in pairs if they want to be competitive. \\
\hline
\end{tabular}




\section{Standing Broad Jump}

The Standing Broad Jump, also called the Standing Long Jump, is a common and easy to administer test to measure the explosive power of the participant's legs.

\section{Equipment:}

- Long Jump Landing Mat

- Recording sheets

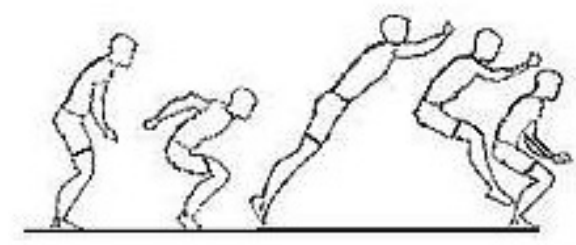

\section{Personnel:}

- $\quad$ 1-2 people

\section{Procedure:}

- The participant stands behind a line marked on the ground with feet slightly apart.

- A two foot take-off and landing is used, with swinging of the arms and bending of the knees to provide forward drive.

- The participant attempts to jump as far as possible, landing on both feet without falling backwards.

- Three attempts are allowed.

\section{Scoring:}

The measurement is taken from take-off line to the nearest point of contact on the landing (back of the heels).

Record the longest distance jumped, the best of three attempts. 


\section{Explaining the Standing Long Jump to children:}

\begin{tabular}{|c|c|}
\hline & Assessment item \\
\hline & Standing Long Jump \\
\hline What will you do? & You will jump 2-footed as far as you can \\
\hline Why? & We want to measure your leg power \\
\hline What will it tell you? & $\begin{array}{c}\text { How far you can jump and how springy you are. This is good for } \\
\text { sports and dance that require leg power }\end{array}$ \\
\hline Is it competitive? & $\begin{array}{l}\text { Yes it is. Make as big an effort as you can. Can you jump your } \\
\text { own height? }\end{array}$ \\
\hline $\begin{array}{l}\text { How will you feel after } \\
\text { it? }\end{array}$ & $\begin{array}{l}\text { You should feel good and springy and you should have a few } \\
\text { attempts to jump as far as you can }\end{array}$ \\
\hline $\begin{array}{l}\text { How can you help } \\
\text { others? }\end{array}$ & Help motivate them if they want you to. \\
\hline Hints for instructors & $\begin{array}{c}\text { Being powerful is important for all sports and dance. } \\
\text { Tell children to use their arms well. } \\
\text { What \% of their height can they jump? How far can they jump } \\
\text { when they keep their arms behind their back for the whole } \\
\text { jump? The difference between hands behind back and not } \\
\text { shows how well they use their arms to transfer momentum into } \\
\text { the jump }\end{array}$ \\
\hline
\end{tabular}


EXAMPLE DATA COLLECTION SHEETS:

(NAME OF PRIMARY SCHOOL)

\section{0m Multistage Fitness Test Recording Sheet}

\begin{tabular}{|c|c|c|}
\hline Participant Number & Last Lap Completed & Notes \\
\hline 1 & & \\
\hline 2 & & \\
\hline 3 & & \\
\hline 4 & & \\
\hline 5 & & \\
\hline 6 & & \\
\hline 7 & & \\
\hline 8 & & \\
\hline 10 & & \\
\hline
\end{tabular}

\section{(NAME OF PRIMARY SCHOOL)}

\section{Speed Bounce Recording Sheet}

\begin{tabular}{|c|c|c|}
\hline Participant Number & $\frac{\text { \# of Correct Bounces (Trial }}{\text { 1) }}$ & \# of Correct Bounces (Trial \\
\hline 1 & & 2) \\
\hline 2 & & \\
\hline 3 & & \\
\hline 4 & & \\
\hline 5 & & \\
\hline 6 & & \\
\hline 7 & & \\
\hline 8 & & \\
\hline 9 & & \\
\hline 10 & & \\
\hline
\end{tabular}

(NAME OF PRIMARY SCHOOL)

Sit \& Reach Recording Sheet

\begin{tabular}{|c|c|}
\hline Participant Number & Distance Reached (to the nearest CM) \\
\hline 1 & \\
\hline 2 & \\
\hline 3 & \\
\hline 4 & \\
\hline 5 & \\
\hline 6 & \\
\hline 7 & \\
\hline 8 & \\
\hline 9 & \\
\hline 10 & \\
\hline
\end{tabular}




\section{EXAMPLE DATA COLLECTION SHEETS:}

\section{(NAME OF PRIMARY SCHOOL)}

Handgrip Dynanometer Strength Test Recording Sheet

\begin{tabular}{|c|c|c|}
\hline Participant Number & Left Hand Reading & Right Hand Reading \\
\hline 1 & & \\
\hline 2 & & \\
\hline 3 & & \\
\hline 4 & & \\
\hline 5 & & \\
\hline 6 & & \\
\hline 7 & & \\
\hline 9 & & \\
\hline 10 & & \\
\hline & & \\
\hline
\end{tabular}

(NAME OF PRIMARY SCHOOL)

$10 \times 5 \mathrm{~m}$ Shuttle Run Test Recording Sheet

\begin{tabular}{|c|c|}
\hline Participant Number & Time Taken to complete 50m \\
\hline 1 & \\
\hline 2 & \\
\hline 3 & \\
\hline 5 & \\
\hline 6 & \\
\hline 7 & \\
\hline 8 & \\
\hline 10 & \\
\hline
\end{tabular}

(NAME OF PRIMARY SCHOOL)

Standing Broad Jump Recording Sheet

\begin{tabular}{|c|c|c|c|c|}
\hline Participant Number & $\underline{1}^{\text {st Jump }}$ & $\underline{2}^{\text {nd }}$ Jump & $3^{\text {rd }}$ Jump & Best Jump \\
\hline 1 & & & & \\
\hline 2 & & & & \\
\hline 3 & & & & \\
\hline 4 & & & & \\
\hline 5 & & & & \\
\hline 6 & & & & \\
\hline 7 & & & & \\
\hline 8 & & & & \\
\hline 9 & & & & \\
\hline 10 & & & & \\
\hline
\end{tabular}


EXAMPLE DATA COLLECTION SHEETS:

(NAME OF PRIMARY SCHOOL)

Weight, Height and Sitting Height Recording Sheet

\begin{tabular}{|c|c|c|c|}
\hline Participant No. & Height $(\mathrm{cm})$ & Sitting Height $(\mathrm{cm})$ & Weight (kg) \\
\hline 1 & & & \\
\hline 2 & & & \\
\hline 3 & & & \\
\hline 4 & & & \\
\hline 5 & & & \\
\hline 6 & & & \\
\hline 7 & & & \\
\hline 8 & & & \\
\hline 9 & & & \\
\hline 10 & & & \\
\hline
\end{tabular}


Appendix III: Child Health and Activity Tool: Online Questionnaire

\section{Personal Details:}

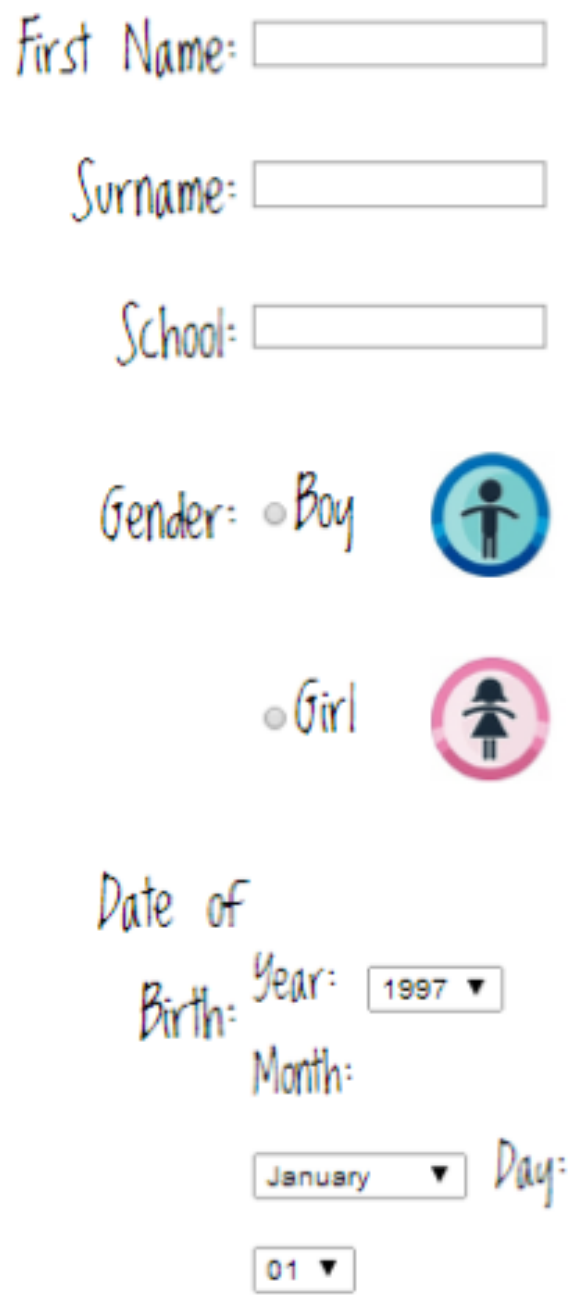

Postcode: 
Do you consider yourself to have a discability?

Yes $\circ$ No

$\square$ Wheekchair user $\square$ Physical disability $\square$ Visual impairment

$\square$ Hearing impairment $\square$ Learning difficulty $\square$ Other

\section{$0 \%$}

Forward $\rightarrow$

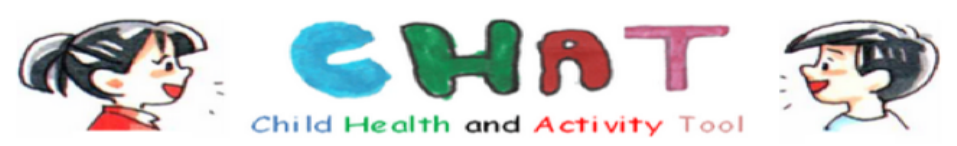

Firstly, think carefully about what you did YESTERDAY and then answer the following questions....

$3 \%$

$\longleftarrow$ Back

Forward $\rightarrow$

1. What time did you get up yESTERDAY?

$\circ 05.00$

○ 05.30

$0 \quad 06.00$

$\circ 06.30$

○ 07.00

- 07.30

$\circ 08.00$

$\circ 08.30$

$\circ 09.00$

- Other Time

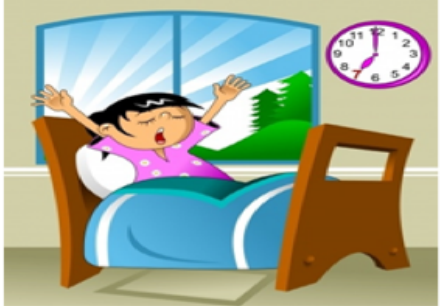


2. What did you eat for breakfast YESTERDAY?

i(1) Nothing

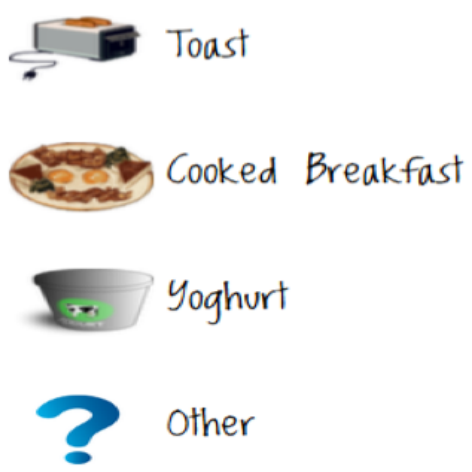

3. What did you have to drink for breakfast?

$\square$ Nothing

$\square$ Water

$\square$ Milk

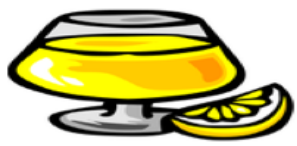

$\square$ Fruit juice e.g. Orange juice, apple juice etc

$\square$ Squash

$\square$ Energy drink e.g. Lucozade Sport, Powerade, Red

Bull

$\square$ Fizzy Drink e.g. Coke, Sprite

$\square$ Diet fizzy drink e.g. Diet Coke, Diet Fanta

$\square$ Other:

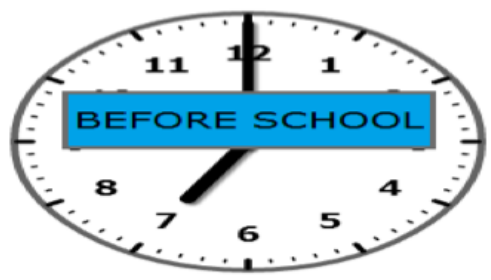

4. a) BEFORE LESSONS STARTED YESTERDAY, how long did you spend doing sports or exercise? $\downarrow$
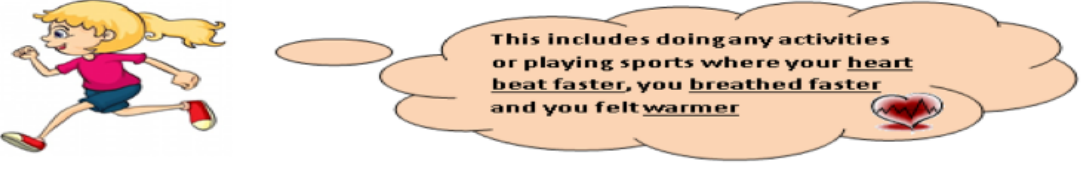


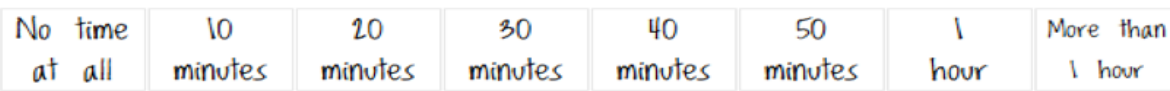

b) BEFORE LESSONS STARTED YESTERDAY, how long did you spend sitting down watching TV/playing computer games/using IPAD or internet? $\downarrow$

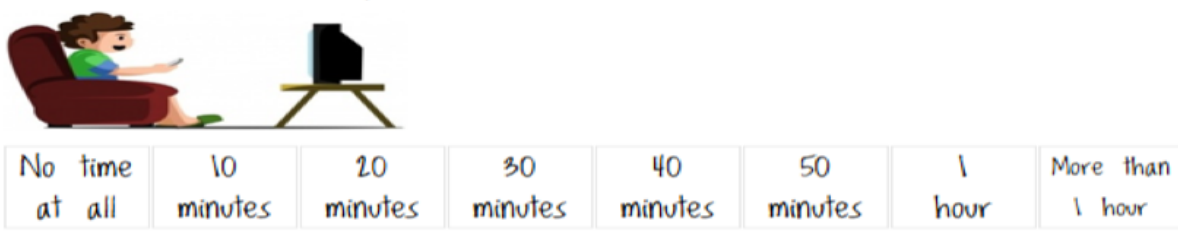

c) BEFORE LESSONS STARTED YESTERDAY, how long did you spend doing homework/reading? $\downarrow$

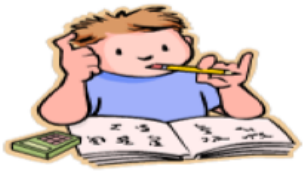

\begin{tabular}{|c|c|c|c|c|c|c|c|}
\hline $\begin{array}{c}\text { No time } \\
\text { at all }\end{array}$ & 10 & 20 & 30 & 40 & 50 & 1 & More than \\
\hline minutes & minutes & minutes & minutes & minutes & hour & 1 hour \\
\hline
\end{tabular}

5. How did you get to school yESTERDAY morning?

On the bus

on bike

00 In the car/taxi

1. Walked

\& Ran/jogged

$\underset{\sim}{\sim}$ Scooter

Skateboarded/Rollerbladed

Did you travel with an adult?

Yes
No


6. What did you do for most of your MORNING break YESTERDAY? Please only tick one option.

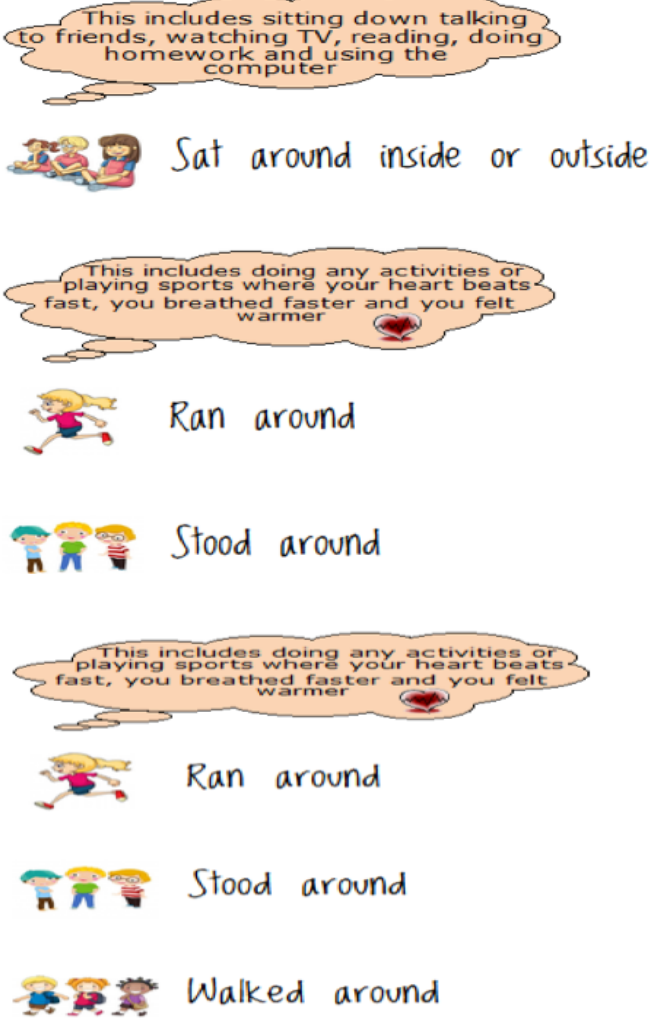

7. a) What did you have to eat for lunch? School Dinner Packed Lunch

2 Nothing

b) What did you have to drink for lunch?

Water

Fizzy Drink

Squash

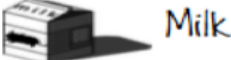

Fruit Juice

Diet Fizzy Drink

Energy Drink

Nothing 


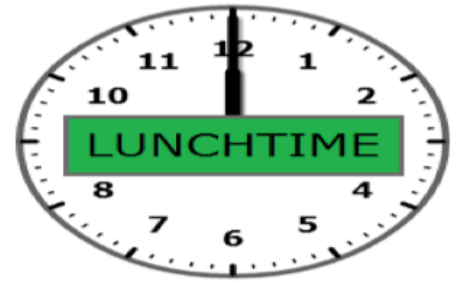

8. Apart from eating your food, what did you do for MOST of your lunchtime YESTERDAY? Please only tick one option.
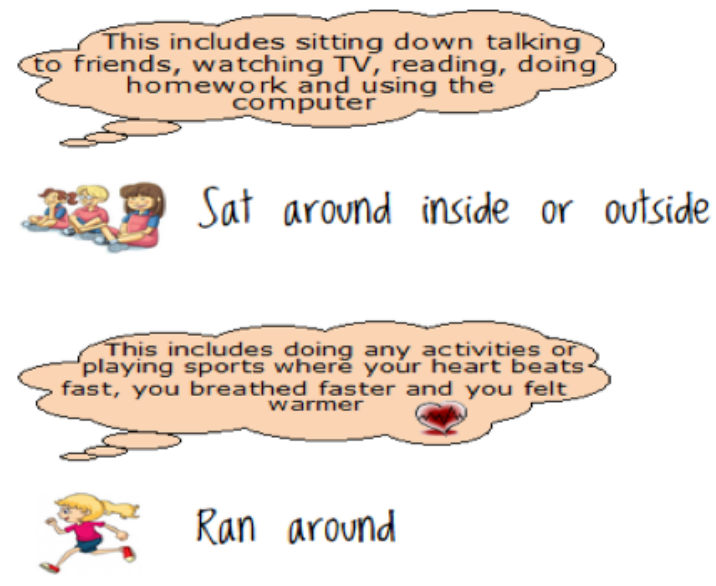

n⿳⺈冂大 Stood around

19. Walked around

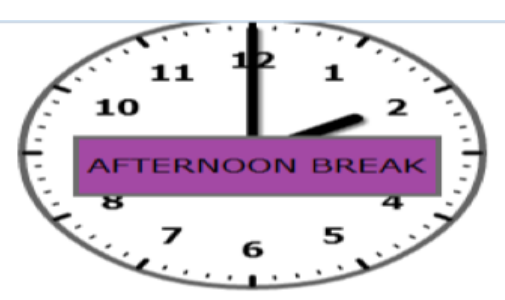

9. What did you do for most of your afternoon break YESTERDAY?

D Didn't have an afternoon break 


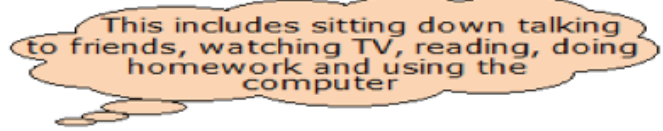

Sat around inside or outside

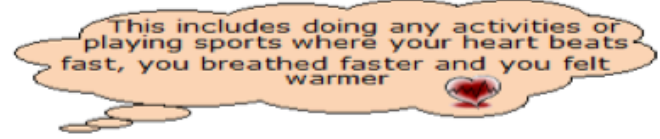

IS: Ran around

के Stood around

19. Walked around

10. How did you get home YESTERDAY?
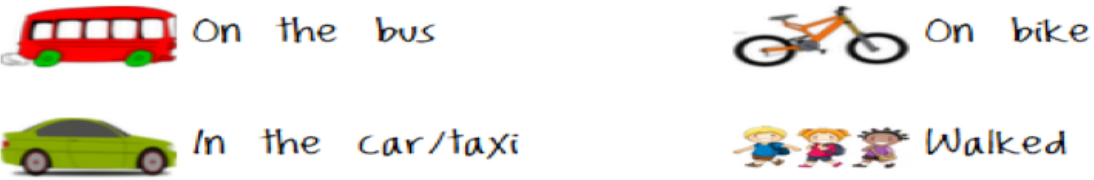

Ran/jogged

Scooter

Skateboarded/Rollerbladed

Did you travel with an adult?

Yes

$\circ$ No 


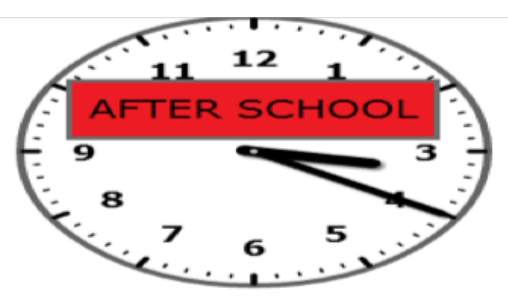

II. a) AFTER SCHOOL YESTERDAY, how long did you spend doing sports or exercise? $\downarrow$
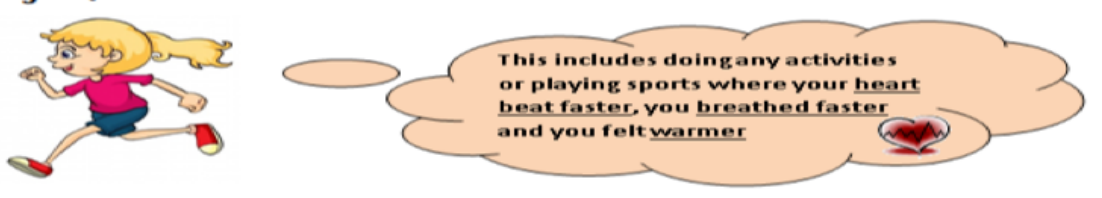

\begin{tabular}{|c|c|c|c|c|c|c|}
\hline $\begin{array}{c}\text { No time } \\
\text { at all }\end{array}$ & $\begin{array}{c}10 \\
\text { minutes }\end{array}$ & $\begin{array}{c}20 \\
\text { minutes }\end{array}$ & $\begin{array}{c}30 \\
\text { minutes }\end{array}$ & $\begin{array}{c}40 \\
\text { minutes }\end{array}$ & $\begin{array}{c}50 \\
\text { minutes }\end{array}$ & $\begin{array}{c}1 \\
\text { hour }\end{array}$ \\
\hline Between 1 & Between 2 & More than & & & & \\
\hline
\end{tabular}

b) AFTER SCHOOL YESTERDAY, how long did you spend sitting down watching TV/playing computer games/using IPAD or internet? $\downarrow$

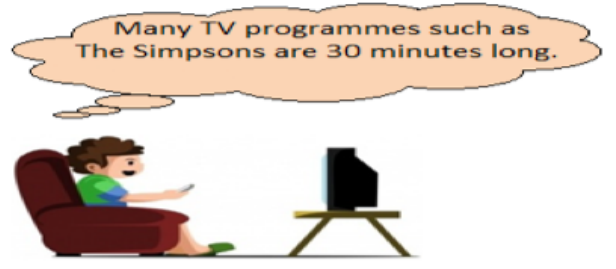

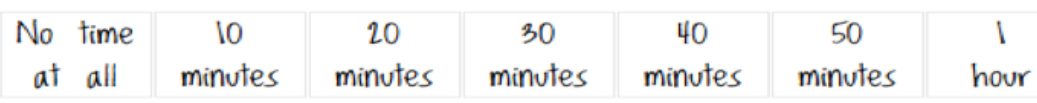

Between 1 Between 2 More than

and 2 hours and 3 hours 3 hours
}

c) AFTER SCHOOL YESTERDAY, how long did you spend doing homework/reading? \

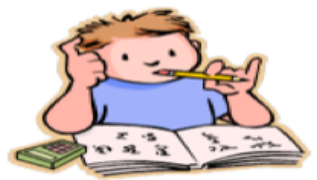

\begin{tabular}{|c|c|c|c|c|c|c|}
\hline $\begin{array}{l}\text { No time } \\
\text { at all }\end{array}$ & $\begin{array}{c}10 \\
\text { minutes }\end{array}$ & $\begin{array}{c}20 \\
\text { minutes }\end{array}$ & $\begin{array}{c}30 \\
\text { minutes }\end{array}$ & $\begin{array}{c}40 \\
\text { minutes }\end{array}$ & $\begin{array}{c}50 \\
\text { minutes }\end{array}$ & $\begin{array}{c}1 \\
\text { hour }\end{array}$ \\
\hline $\begin{array}{l}\text { Between } 1 \\
\text { and } 2 \text { hours }\end{array}$ & $\begin{array}{l}\text { Between } 2 \\
\text { and } 3 \text { hours }\end{array}$ & $\begin{array}{c}\text { More than } \\
3 \text { hours }\end{array}$ & & & & \\
\hline
\end{tabular}


12. How many portions of fruit and vegetables did you eat yesterday?

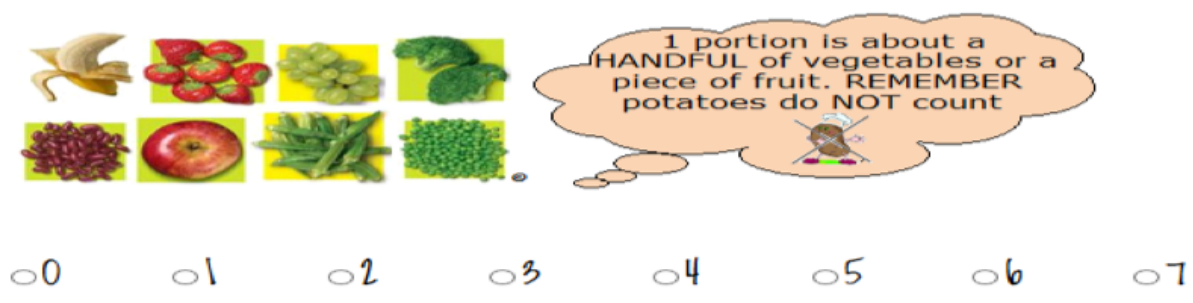

13. How many times did you brush your teeth yesterday?

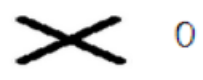

14. What time did you go to sleep?

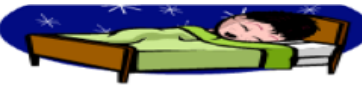

$\begin{array}{cl}07 \mathrm{pm} & 07.30 \mathrm{pm} \\ 08 \mathrm{pm} & 08.30 \mathrm{pm} \\ 09 \mathrm{pm} & 09.30 \mathrm{pm} \\ 010 \mathrm{pm} & 010.30 \mathrm{pm} \\ 011 \mathrm{pm} & \circ 11.30 \mathrm{pm} \\ 012 \mathrm{am} & \text { Other Time: }\end{array}$

Well done you are half way through! 
15. Now think about what you did in the last WEEK..... (INCLUDING THE WEEKEND)
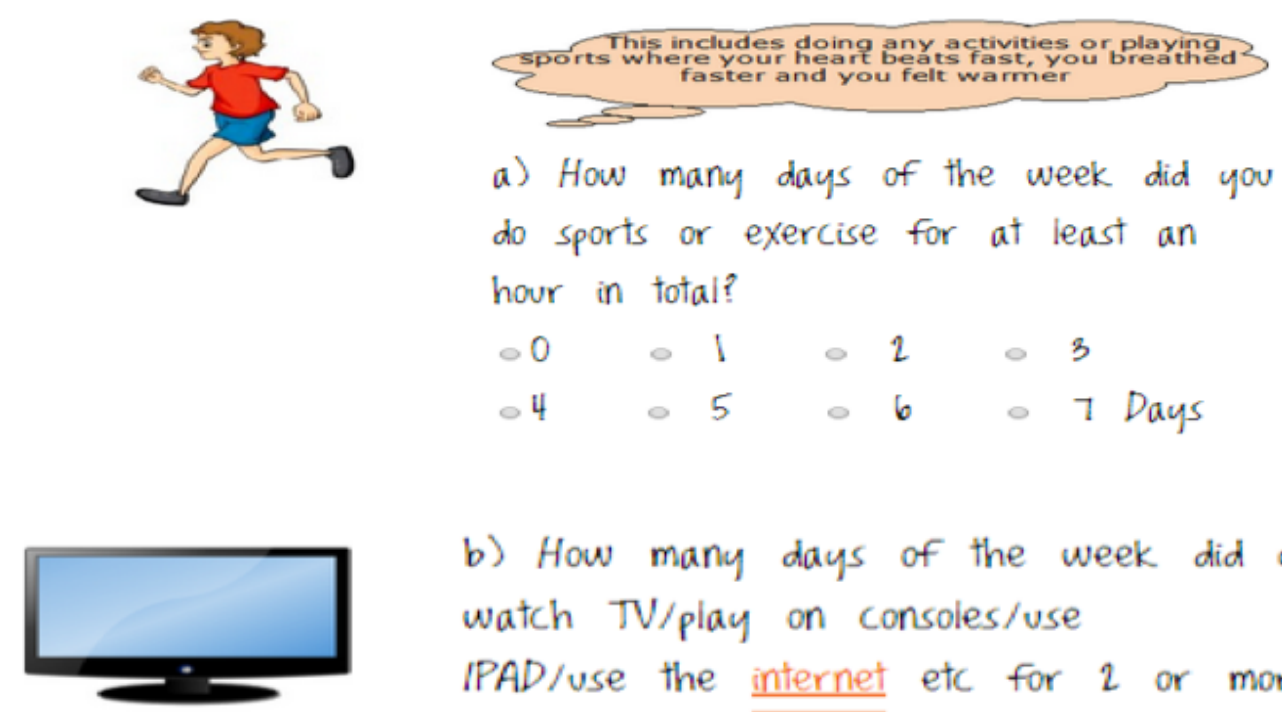

a) How many days of the week did you do sports or exercise for at least an hour in total?

$\begin{array}{llllll}0 & -1 & 0 & 2 & - & \\ -4 & \circ & 0 & 0 & 7 \text { Days }\end{array}$

b) How many days of the week did you watch TV/play on consoles/use IPAD/use the internet etc for 2 or more hours a day?

$\begin{array}{ccccc}0 & 1 & 0 & 2 & 3 \\ -4 & 5 & \circ & 0 & 7 \text { Days }\end{array}$

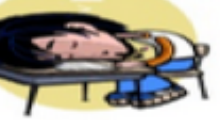

c) How many days of the week did you feel tired?
$\circ 0 \div 1 \div 3$
$04 \div 5 \div 6$ D 5 Days 


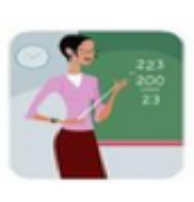

d) How many days of the week did you feel like you could concentrate/focus well in class?

$$
\begin{aligned}
& \circ \circ 1 \circ 2 \circ 3 \\
& \text {-4 } 5 \text { Days }
\end{aligned}
$$

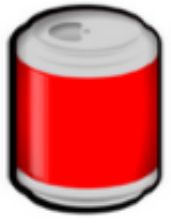

e) How many days of the week did you drink at least one fizzy drink e.g. coke, fanta, sprite?

$$
\begin{array}{ccccc}
0 & 1 & \circ & 3 \\
-4 & 5 & \circ & \circ & 7 \text { Days }
\end{array}
$$

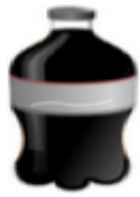

f) How many days of the week did you drink at least one DIET fizzy drink e.g. Diet coke, diet fanta?

$$
\begin{array}{llll}
0 & 1 & - & 2 \\
-4 & 5 & \circ & 3
\end{array}
$$

g) How many days of the week did you eat at least one sugary snack e.g. sweets/chocolate/cake?
$\circ 0$

1
- 2
$\circ 3$
$\circ 4$
- 5
- 6 - 7 Days 


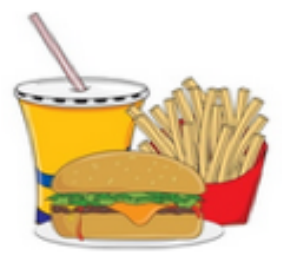

h) How many days of the week did you eat take away foods e.g. McDonalds,

$\mathrm{KFC}$, chinese?
$\circ 0$
$\circ 1 \circ 2 \circ 3$
$\circ 4$
$\circ 5$
$\circ 6$
- 7 Days

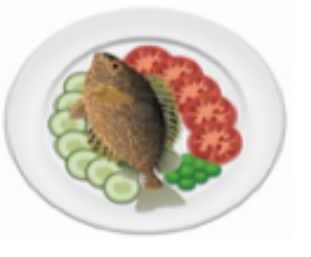

i) How many days of the week did you eat fish?
$\circ 0$
$\circ 1$
$\circ 2$
3
$\circ 4$
$\circ 5$
$\circ 6$
- 7 Days

16. What type of bread do you eat?

$C^{\prime}$ I don't eat bread

2 Brown/wholemeal
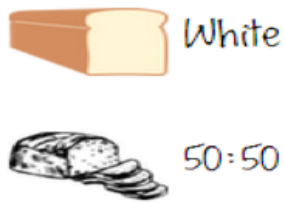

17. What type of milk do you drink?

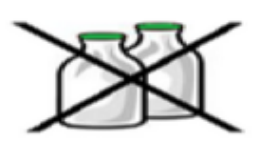

I don't drink milk

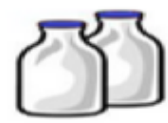

Whole milk

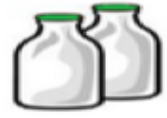

Semi-skimmed milk

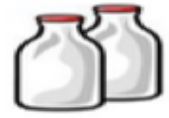

Skimmed milk 
18. What sport clubs or physical activities do you take part in at least once a week?

IN SCHOOL: (this does not include PE)
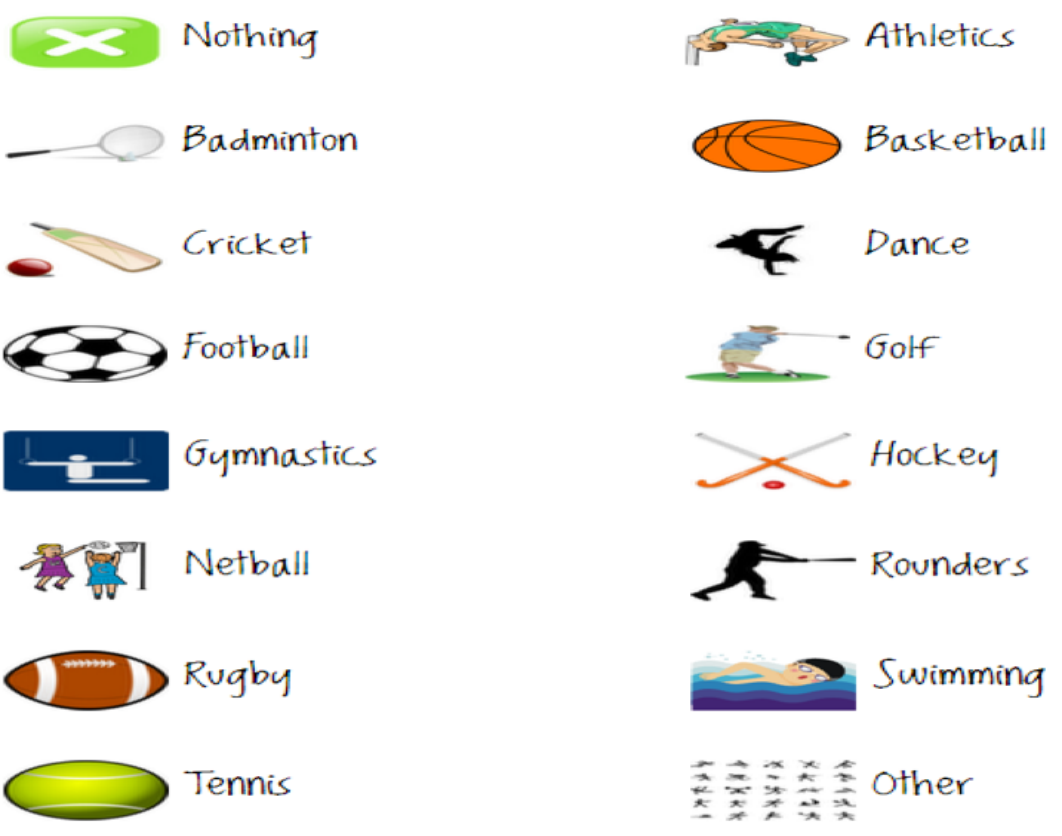

OUT Of SCHOOL:

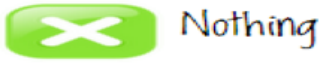

Badminton

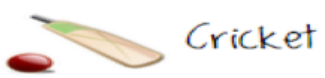

$\%$ Dance
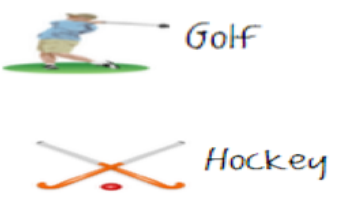

स्विए। Netball
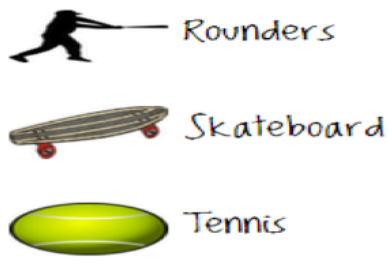

Water Sports
Athletics
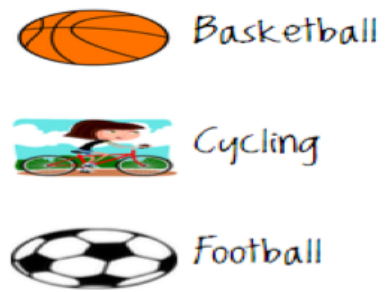

- Gymnastics
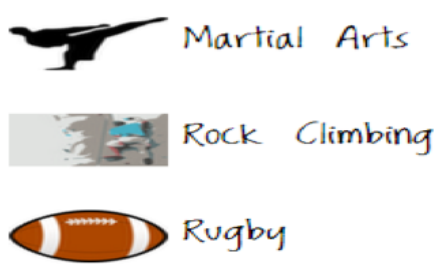

$\rightarrow$ Swimming

Trenpolining

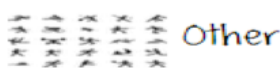


19. Are you a member of cubs, brownies, guides or scouts?

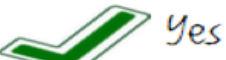

20. WHO do you usually take part in physical activity or sport with?

IN SCHOOL:

i. On your own

with Friends

With Parents/Guardians

With Brother/Sister

2 Other:

OUT OF SCHOOL:

i. On your own

With Friends

With Warents/Guardians

2 With Brother/Sister

2 other: 
21. How many times a week do your parents/guardians do exercise or sport (This includes going for a run or going to the gym)?

Parent/Guardian 1: $\quad$ Fi Female (i) Male
- Not at all
$\circ 1-2$
$3-4$
- 5 or more

Parent/Guardian $2:$
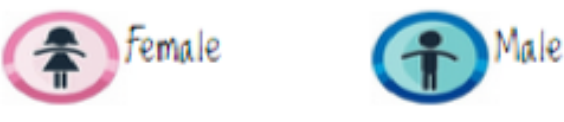

(Ootionai)
- Not at all
$\circ 1-2$
$-3-4$
.5 or more

22. Where can you play at home/in the surrounding area that you can walk to?

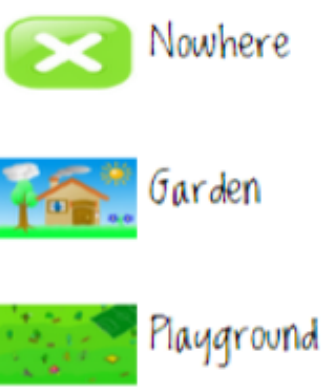

Firis on the street

School

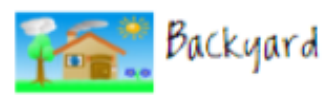

Whidareg Grassy area/playing

field

en= Park

Inivin- Leisure/sport centre 
23. Which of these physical activities or sports would you like to do more of?

Ponletics

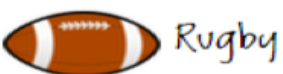

$\longrightarrow$ Tennis

$\rightarrow$ Swimming

8 Other

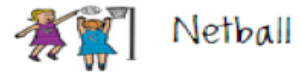

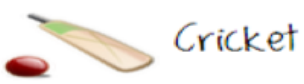

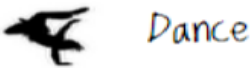

2 None of these

24. What physical activities or sports would you like to take part in that you have not tried before?

H

25. Can you ride a bike?

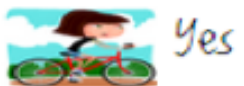

$2 N_{0}$

26. a) Can you swim?

$\rightarrow$ Yes

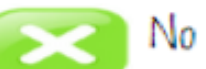

26. b) Can you swim 25 metres?

Yes

No 
27 How do you feel about:
a) Your heaith:
(2) 20
b) Your fitness:

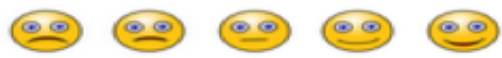
c) Your schooi:
(2) 2000
d) Your family:
(2) 2000000
e) Your friends:
(2) 200

28. Now tell us whether you agree or disagree with the following

a) I am doing weil at schooi:
Strongly
Disagree
Don't agree
Agree
Strongly
disagree
or disagree
agree
b) I have iots of choice over things which are important to me
Strongly
Disagree
Don't agree
disagree
or disagree
Agree
$\times$
$x$
c) There are iots of things 1 am good at

Strongly

agree

$\begin{array}{ccccc}\text { Strongly } & \text { Disagree } & \begin{array}{c}\text { Don't agree } \\ \text { or disagree }\end{array} & \text { Agree } & \text { Strongly } \\ \text { disagree } & & & & \text { agree } \\ \times & \times & & \end{array}$

29. What do you think could be done in your local area to improve the health of children? 

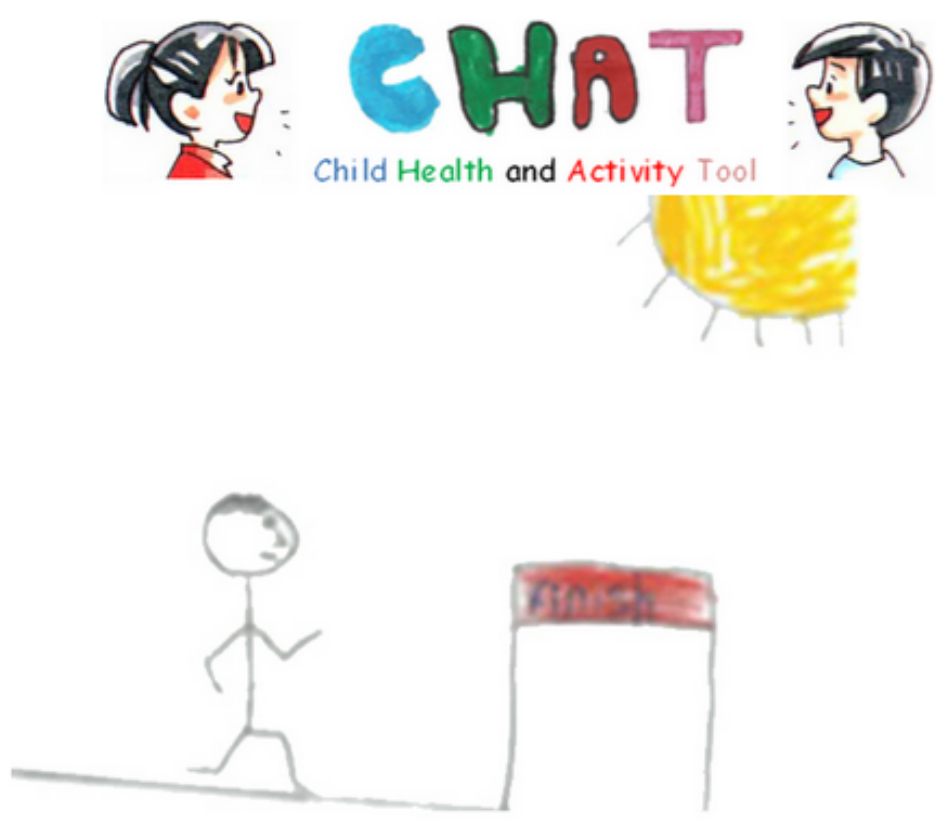

Well done, you've completed the questionnaire.

Thank you!

Start again

$100 \%$ 
Appendix IV: Standard Operating Procedure for Child Health and Activity Tool
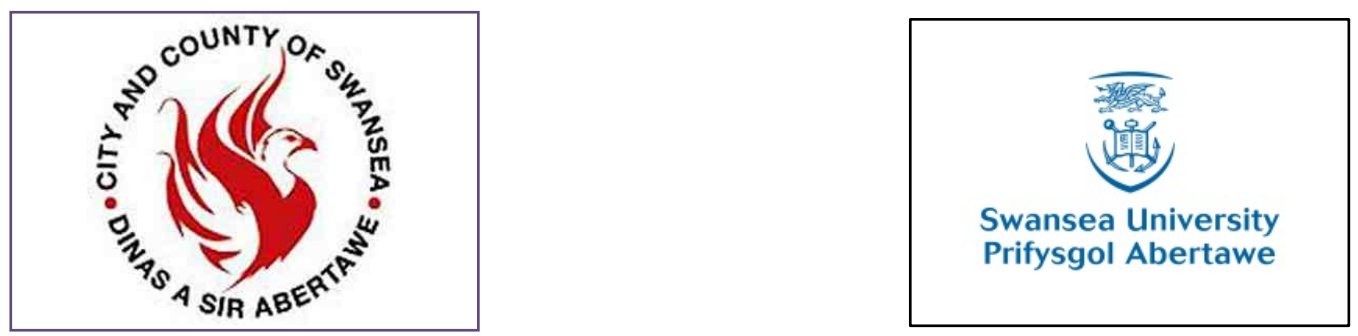

Standard Operating Procedure

For

\section{Child Health and Activity Tool (CHAT) \\ www.childactivity.co.uk}




\subsection{INTRODUCTION}

This section introduces the standard operating procedure (SOP) to administer the Child's Health and Activity Tool (CHAT). CHAT has been designed as an online health and lifestyle questionnaire for primary school children. The data collected from CHAT will be used alongside fitness test data to determine the health and fitness parameters of children. CHAT is compatible with iPads and laptops and is available in English and Welsh. This SOP will explain in detail how a researcher should administer CHAT to a class of children. The SOP includes instruction for:

\subsection{Information prior to administering CHAT.}

\subsection{Administering CHAT.}

1.3 Accessing the data after administering CHAT.

\subsection{PRIOR TO ADMINISTERING CHAT}
1) Date and time
2) IT Facilities
3) Postcodes

\section{1) Arrange date and time for questionnaire to take place}

- Contact the school 2-3 weeks in advance of administering CHAT and gauge interest in taking part (phone call and standard recruitment email).

- Send out head teacher, parent and participant information sheets and consent forms to schools.

- CHAT can't be administered on a Monday or after a Bank Holiday due to questions asking what the children did yesterday (also avoid administering on weeks focused on health and exercise, such as walk or cycle to school week etc.).

- Find out how many classes will be completing CHAT.

- Find out the number of pupils in each class.

- Allow minimum of 45 minutes for a class to complete CHAT in one sitting. This allows for introduction and explanation, the children to complete CHAT, and thanks at the end.

- Remember to ask about break and lunchtime schedules.

- Ask the teacher beforehand if there is anything the children do once they finish CHAT as some children will finish sooner than others. These other activities may be educational online games or other work that they have been doing.

\section{2) IT Facilities}

- Ensure there are enough computers/iPads for all children in class to complete CHAT at the same time. Otherwise class will need to be split.

- Ask about the IT facilities that the school has. Does the school have Wi-Fi and use iPads or laptops or does it have an IT suite where the computers all have a local connection. 
- If the children will be using iPads or laptops, ensure they will all have been charged beforehand and check wireless network connection and what usernames and passwords are required.

- If the IT facilities (IT suite, laptops, iPads) need to be booked make sure this is done in advance.

- Clarify that Google Chrome is the best internet browser to administer CHAT.

\section{3) Postcodes:}

- Ask the teacher to bring several copies of sheets with the children's postcodes.

- If unwilling to bring sheets then ask to bring student info folder from main office.

- Have all children who don't know their post code raise their hand at the start of CHAT and then wait for their teacher to come over and tell them.

\subsection{ADMINISTERING CHAT}

\section{4) Introduction}

5) If CHAT stops working

6) Start page

7) CHAT Questions 1-29

\section{4) Introduction:}

- Before administering CHAT explain what it is about.

"It asks questions on how much exercise you did, what you ate, what time you went to sleep and how you feel about certain things. The first part follows a timeline of what you did yesterday from when you woke up to when you went to sleep. You can follow the clock at the top for idea of time. So, think carefully about what you did yesterday before answering. Some questions look similar but if you read it closely it's asking about different section of day (the clock on top helps with this). There are no right or wrong answers but it is important you answer all questions honestly. Read the question slowly to make sure you understand it properly and do not rush - it's not a race. If you don't understand a question, see if there is a speech bubble which normally explains a bit further. If you have read the speech bubble and still don't understand, put up your hand and wait for the teacher to explain. If ill or on holiday, think of the last normal school day when you were in school".

- Log on to www.childactivity.co.uk.

- Bilingual: Option to complete in welsh by clicking "cymraeg" in top right corner.

\section{5) Problem}

If CHAT freezes or will not let you go forward, try and click the refresh button by the web address. If this does not work or the pupil closes the CHAT window, they will have to start from the beginning. If they had got further than 3 or 4 questions, have a researcher take note of the child's previous progress, help them fill in the first page correctly and then click any answer for the questions that they had already completed. Once they reach where 
CHAT had previously frozen, the child can carry on with completing CHAT. The data will then have to be edited manually by a researcher after CHAT is completed.

\section{6) Start Page}

If they don't know if they have a disability ask them to go through each of the disability options to see if any apply to them, if they don't have any then tell them to click no.

\section{7) CHAT Questions 1-29}

\section{Question 1) What time did you wake up?}

- Choose closest time or click other for more specific (choose time they woke up not got up).

\section{Question 2) What did you eat for breakfast?}

- Healthy and unhealthy cereal (if unsure click other at bottom and put the name of the cereal).

- Sometimes the other box will appear higher on the page.

\section{Question 3) What did you drink for breakfast?}

- Children can click more than one option.

Question 4) Before school how long did you spend..

a) Doing sport or exercise?

- Make sure children read the speech bubble (Brisk walking would count but not slow/normal walking).

b) Sitting down watching TV/playing video games/using iPad/internet?

- Ask them to use length of TV programmes as guide and to make sure they add up each sedentary activity.

c) Doing homework or reading?

- Reading after school has started does not count.

\section{Questions 5) How did you get to school?}

- If the children say they did more than one option, ask them which they spent the most of their journey doing.

- If they travelled with an adult, ask if they travelled most of the way with them? If so, then YES but if not then NO.

\section{Question 6) What did you do for most of your morning break?}

- Make sure the children read the speech bubbles above "sit around" and "ran around" for examples.

Question 7) What did you have to..

a) Eat for lunch?

b) Drink for lunch 


\section{Question 8) What did you do for most of lunchtime apart from eating food?}

- Make sure children read the part of the question explaining that the period of time eating lunch does not count.

- Make sure the children read the speech bubbles above "sit around" and "ran around" for examples.

\section{Question 9) What did you do for most of your afternoon break?}

- Make sure the children read the speech bubbles above "sit around" and "ran around" for examples.

\section{Questions 10) How did you travel home from school?}

- If the children say they did more than one option, ask them which they spent the most of their journey doing.

- If they travelled with an adult, ask if they travelled most of the way with them. ? If so, then YES but if not then NO.

\section{Question 11) After school how long did you spend..}

a) Doing sport or exercise?

- Make sure children read the speech bubble (Brisk walking would count but not slow/normal walking).

b) Sitting down watching TV/playing video games/using iPad/internet?

- Ask them to use length of TV programmes as guide and to make sure they add up each sedentary activity.

c) Doing homework or reading?

\section{Question 12) How many portions of fruit and vegetables did you eat yesterday?}

- Remember potatoes don't count.

- Follow the link below to see what amount of fruit and vegetables counts as a portion:

http://www.nhs.uk/Livewell/5ADAY/Documents/Downloads/5ADAY_portion_guid e.pdf.

Question 13) How many times did you brush your teeth yesterday?

- Remember to ask whether children brush their teeth at school as well.

Question 14) What time did you go to sleep?

- Choose closest time or click other for more specific (choose time they went to sleep not went to bed).

Question 15) How many days a week..

a) Did you do sport or exercise for 1 hour or more?

- Make sure children read the speech bubble 
- Includes weekdays and weekends

b) Did you watch TV/play on consoles/use iPad/use the internet for 2 hours or more?

- Make sure children realise it is 2 hours or more in total, not for each activity.

c) Did you feel tired?

- Make sure children understand it means all day, not just drowsy in the morning or at night.

d) Did you feel like you could concentrate/focus in class?

- If children don't understand concentrate/focus use alternatives. For example: "pay attention to what the teacher is saying during class."

e) Did you drink one fizzy drink?

- Remember to remind the children this is how many days a week not how many fizzy drinks.

- It only includes full sugar drinks, for example: Coca Cola not Coca Cola Diet or Coca Cola Zero.

f) Did you drink one diet fizzy drink?

- Remember to remind the children this is how many days a week not how many diet fizzy drinks.

- Includes zero sugar drinks such as Coca Cola Zero/Fanta Zero as well as diet fizzy drinks.

g) Did you eat one sugary snack?

- Remember to remind the children this is how many days a week not how many sugary snacks.

- This includes cakes, sweets and chocolate.

h) Did you eat a takeaway?

- This includes all fast food takeaways.

i) Did you eat fish?

- This includes all types of fish, including fresh, battered and tinned.

\section{Question 16) What type of bread do you eat?}

- If children have more than one type of bread, ask them to choose which one they have the most.

\section{Question 17) What type of milk do you have?}

- If children have more than one type of milk, ask them to choose which one they have the most.

- If they have rice/soy milk, select "I don't drink milk."

\section{Question 18) What sports/activities do you take part in..}

a) In school?

- Children should only select sports/activities that are not part of the curriculum. 
- They should only select sports/activities that are extra-curricular (such as school teams) or sports/activities that they do at breaktime/lunchtimes.

- The sports/activities should be something they take part in on a regular basis (such as once a week).

b) Outside of school?

- The sports/activities should be something they take part in on a regular basis (such as once a week).

\section{Question 19) Are you a member of brownies/cubs/guides/scouts?}

- If the children are not members at present, then click "No."

\section{Question 20) Who do you usually take part in physical activity or sport with..}

a) In school?

- If children say they don't take part in any physical activity or sport, then get them to select "other" and type "don't do any" in.

- If children say they take part in physical activity or sport with more than one than one group or person, ask them to select the option they take part in physical activity or sport with most.

b) Outside of school?

- If children say they don't take part in any physical activity or sport, then get them to select "other" and type "don't do any" in.

- If children say they take part in physical activity or sport with more than one than one group or person, ask them to select the option they take part in physical activity or sport with most.

Question 21) How many time a week do your parents/guardians do exercise or sport?

- Select gender of first parent/guardian, then how many times a week they do exercise or sport.

- Second parent is optional.

\section{Question 22) Where can you play at home or in surrounding area within walking distance?}

- Children can select as many options as they like.

- School only counts as an option if the school's grounds are open outside of school hours.

- Backyard just relates to non grassy space.

- Garden is a grassy space.

Question 23) Which of these physical activities or sports would you like to do more of?

- Children can select more than one option. 


\section{Question 24) What sports or physical activities would you like to take part in that you}

have not tried before?

- If children are having difficulty thinking of an answer, ask them "have you ever seen something on TV which you would like to try or on the Olympics or your friend/family has taken part in."

\section{Question 25) Can you ride a bike?}

- Stabilisers don't count

\section{Question 26)}

a) Can you swim?

- This doesn't specify distance, but whether they can swim unaided without touching the sides.

b) Can you swim 25 metres?

- If children ask what this means, ask them if they have a $25 \mathrm{~m}$ badge? If not ask them if they can swim a length on their own, unaided, without armbands not touching sides (not of the national pool though-this would be half the length of that).

\section{Question 27) How do you feel about.}

a) Your health?

- Health is overall condition of their body, including diet, fitness, free from illness etc.

b) Your fitness?

- This question relates to how children feel about their bodies when they are performing sports and exercise. For example, do they get out of breath easily etc?

c) Your school?

- How do children feel about how their school overall? This includes teachers, other children and their school performance.

d) Your family?

- How do children feel about their family? This includes parents and siblings.

e) Your friends?

- How do children feel about their friends in school and outside of the school.

Question 28) Do you agree or disagree with..

a) "I am doing well at school"

- This is asking about how children feel they are doing performing academically at school.

b) "I have lots of choice over things that are important to me"

- Ask the children what is important to them personally such as diet, sports, playing with friends, bedtime, what they watch on TV etc and whether they have much choice over it.

c) "There are lots of things I am good at" 
- Give examples if needed. For example: school, musical instrument, sports etc.

Question 29) What do you think could be done to improve the health of children in your local area?

- If children are unsure, make suggestion, such as parks, cycle lanes, youth clubs, local sports clubs etc.

\subsection{ACCESSING THE DATA AFTER ADMINISTERING CHAT}

8) Accessing The Data After Administering CHAT

Steps:

- Log onto: http://www.childactivity.co.uk/login.php

- Username: AVAILABLE UPON REQUEST

- Password: AVAILABLE UPON REQUEST

- Click "Submit"

- Click "Download"

- Open up the Excel document.

- Copy and Paste school's data into new Excel document. 
Appendix V: Dragon Challenge Manual

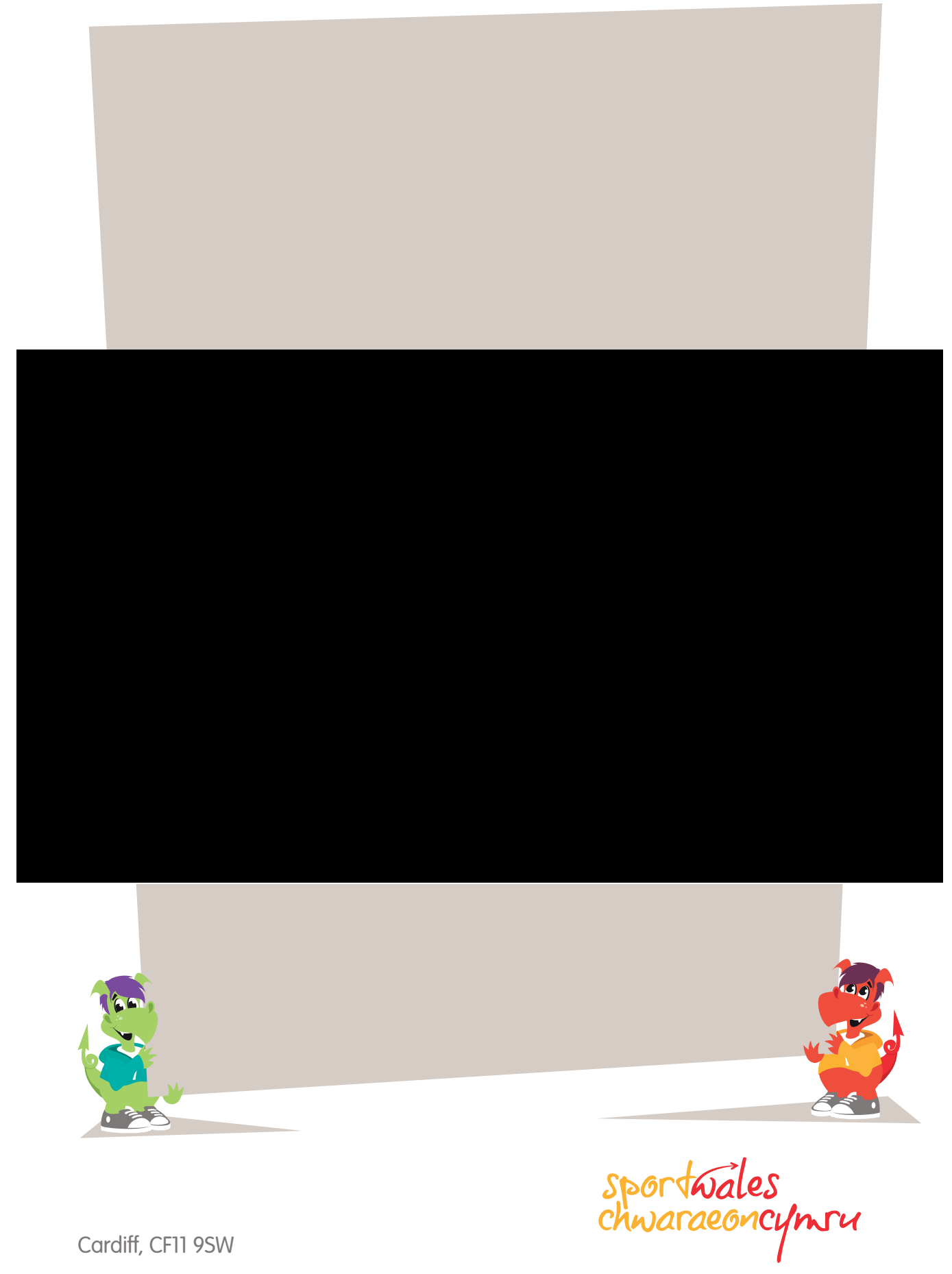




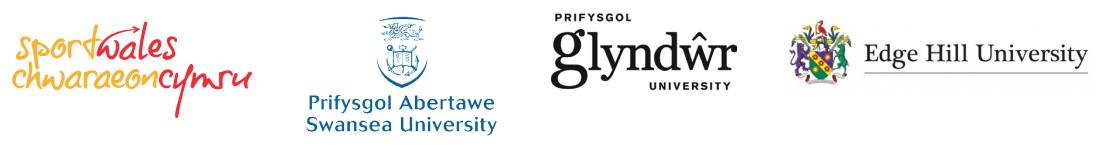

This manual has been produced by Professor Gareth Stratton (Swansea University) and Dr Lawrence Foweather (Edge Hill University) on behalf of Sport Wales.

How to reference this manual:

Stratton G, Foweather, L, Rotchell J, English J, Hughes H (2015). Dragon Challenge V1.0 Manual. Sport Wales. 


\section{Contents}

Acknowledgements

Step 1 - Preparation.

.13

Step 2 - Introduction

. .13

Step 3 - Demonstration of Dragon Challenge

Step 4 - Opportunities for Practice

.16

Step 5 - During the Assessment

17 


How to Record and Assess a Child's Dragon Challenge Performance
Child Performance Record
Assessment Criteria.
Time to Completion

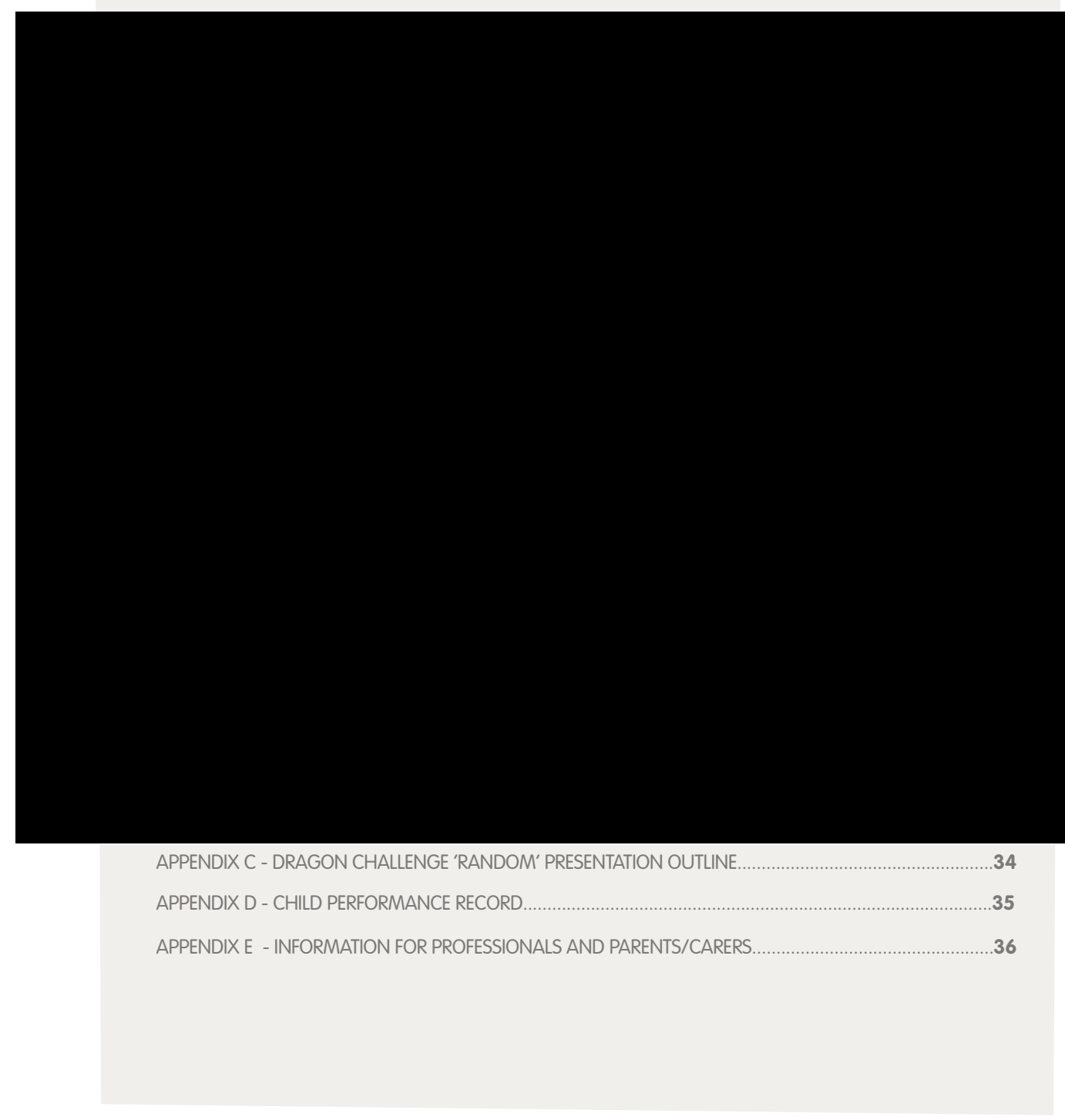




\section{Sackground and Overview}

The clock is ticking. The health and well-being of our nation is at crisis point and there has never been a more critical time to ensure children have a healthy, positive development. The Welsh Government has long recognised that the quantity and quality of physical activity and physical literacy, respectively, is paramount to shaping the health and well-being of children; Wales was the first country to legislate for Play (2002), the first to introduce an

Physical competence is gradually acquired through a complex process of brain and neuromuscular development. The development of physical competence occurs sequentially, progressing from reflexive movements in the first few months of life to the acquisition of a broad repertoire of sport- and contex specific movements in middle to late childhood, adolescence and adulthood (Gallahue et al., 2011). When a child is learning to read they first learn words such as cat, sat, mat. Similarly as a child learns physical skills they learn skills such as how to run, jump, throw and balance. Children then string words together into sentences and read them. In the same way, physical skills are linked together to create movement and perform activities such as riding a bike, swimming or performing the long jump. 


\section{The Importance of Developing Physical Competence}

Physical or motor development is considered to be an important dimension of child development and in the development of cognition (Piaget, 1952). From an early age, the capacity to move gives children the ability to explore and interact with their physical world.

Research suggests that children and adolescents who develop high motor competence are more likely to participate in physical activity (Foweather et al., 2014; Holfelder \& Schott, 2014; Lubans et al.,

sport from across Wales, the assessment tool was further developed and refined until Dragon Challenge Version 10 was finalised It is expected that this version will be revised and updated according to the data and user feedback.

The Dragon Challenge is designed to be a dynamic and engaging assessment tool that provides a developmentally appropriate challenge of children's physical competence. The following sections outline the Dragon Challenge V1.0 and describe the tasks and preparatory procedures, the assessment process and criteria, as well as data management and the interpretation of results. 


\section{Description or the Dragr Challenge} Mapped to Multi-Skill and Multi-Sport Activity Cards

\section{Purpose}

- The purpose of the Dragon Challenge is to assess children's physica

competence.

\section{Target Population}

- Dragon Challenge has been designed for children in school years 6 and 7 (aged $10-12$ years)

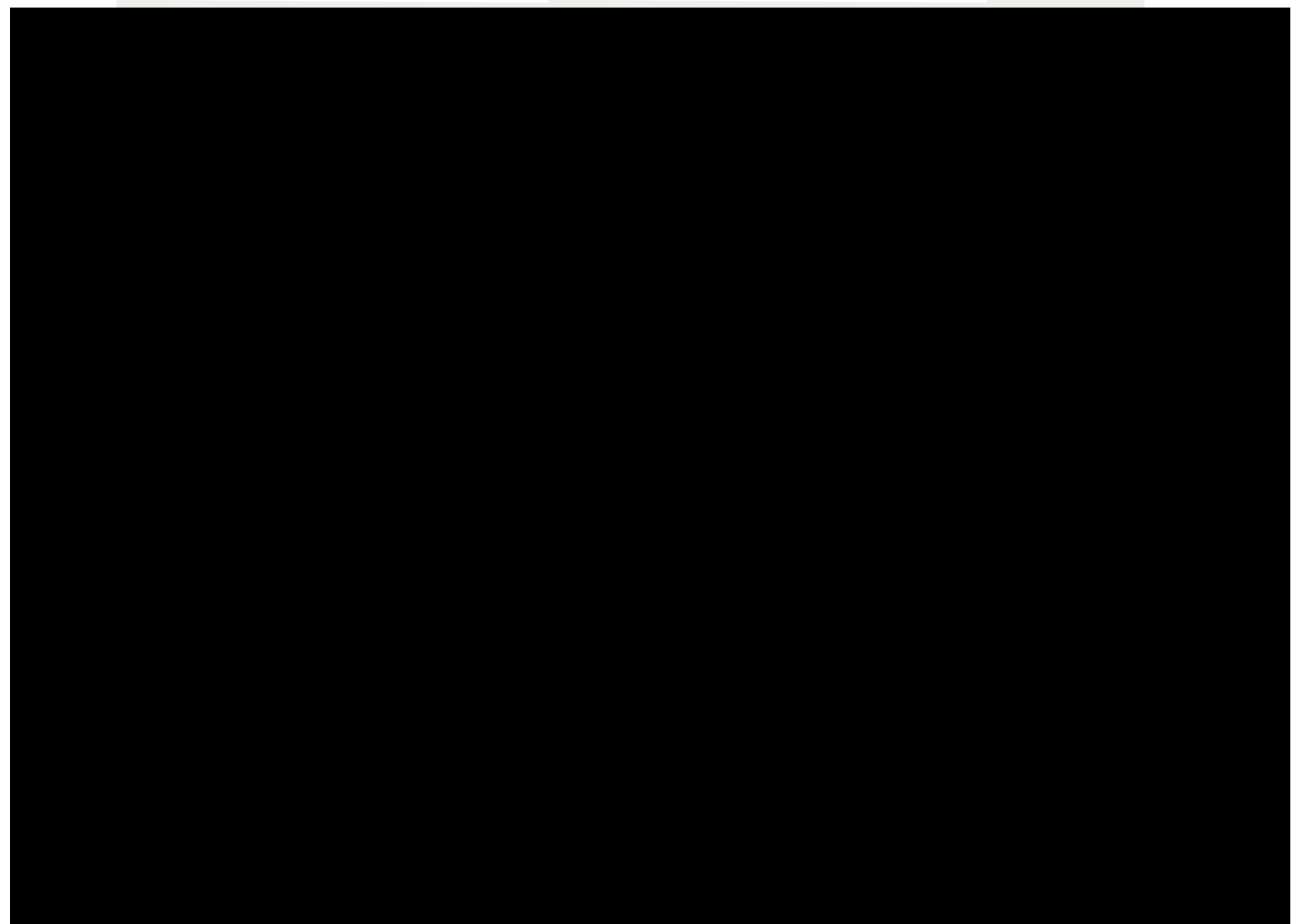

distance.

7. Jumping Patterns

Complete a jumping pattern sequence that includes a series of hops and jumps $(2$ footed jump over hurdle $>2$ footed landing $>2$ left hop $>2$ right hops $>2$ foot jump over hurdle $>2$ footed landing).

8. T-Rgility Complete t-agility run, facing forwards throughout.

9. Iccelerate-Sprint

$10 \mathrm{~m}$ acceleration to a sprint over finish line. 
Table 2. Types of physical skills utilised during each Dragon Challenge assessment task

$\begin{array}{lll}\text { Task } & \text { Body } & \text { Locomotion Manipulative } \\ & \text { Management } & \end{array}$

1. Balance Bench

-

O

2. Core Igility

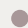

Uses of the Dragon Challenge

The Dragon Challenge assessment has primarily been developed as a surveillance tool to assess patterns and trends in the levels of physical competence among children across Wales. Dragon Challenge data will also become a "Key Performance Indicator (KPI)" for Sport Wales "Every Child Hooked on Sport" and Physical Literacy strategies. 


\section{Treparing Tor the Dragon Challenge rissessment}

This section discusses important matters that should be considered before administering the Dragon Challenge Assessment.

\section{Video Resources}

A series of training videos are available to familiarise instructors and assessors with the challenge and its constituent activities. Video material is presented in a real context and guidance on preparation

Assessor lsee page 29].

Administrators of the Dragon Challenge should also receive basic training. This may include

practicing administering the Dragon Challenge while being observed by an experienced administrator. Gold Standard Assessors will be able to provide information on training 


\section{Child Data}

Each child's Dragon Challenge assessment data needs to be input into an excel data sheet by the assessorls) (see pages $x$ to $x$ for a detailed overview of data organisation and management). In preparation for the assessment, schools will need to provide pertinent information relating to individual child characteristics including:

- Date of birth

- School year

The equipment required to run the Dragon Challenge is listed in Table 3. It is important that only the equipment listed is used and that guidelines relating to the air pressure of the basketball and wobble spot are adhered to; the assessment was standardised using this equipment and any change /improvisation may invalidate the results. Most of the equipment used can be found in primary or secondary schools or is available from Physical Literacy Programme for Schools teams. The availability of spare equipment such as balls and bean bags should also be considered. An iPad or other tablet is required and should be pre-loaded with the Dragon Challenge keynote/ppt. presentation slides that display the order of activities (see Appendix B and C). 
Staffing

A team of two assessors and 1 administrator are required to run the Dragon Challenge. It is desirable that both males and females are represented on the team. At least one member of this team will need to give demonstrations. In groups, an additional member of staff (e.g. physical education teacher) will be needed to run activities to occupy children while they wait for their individual assessment. With additional staffing and space, multiple children can be assessed simultaneously.

$2 \times$ Small traffic cones

$1 \times$ Hula hoop

$1 \times$ Basketball (size 5; 0.5-0.6 bar; 7-9 PSI)

$2 \times$ Tennis balls

$1 \times$ IPad/tablet, fully charged and with Dragon Challenge presentations uploaded (Appendix B and C) 
Figure la Floor Plan Ledgend

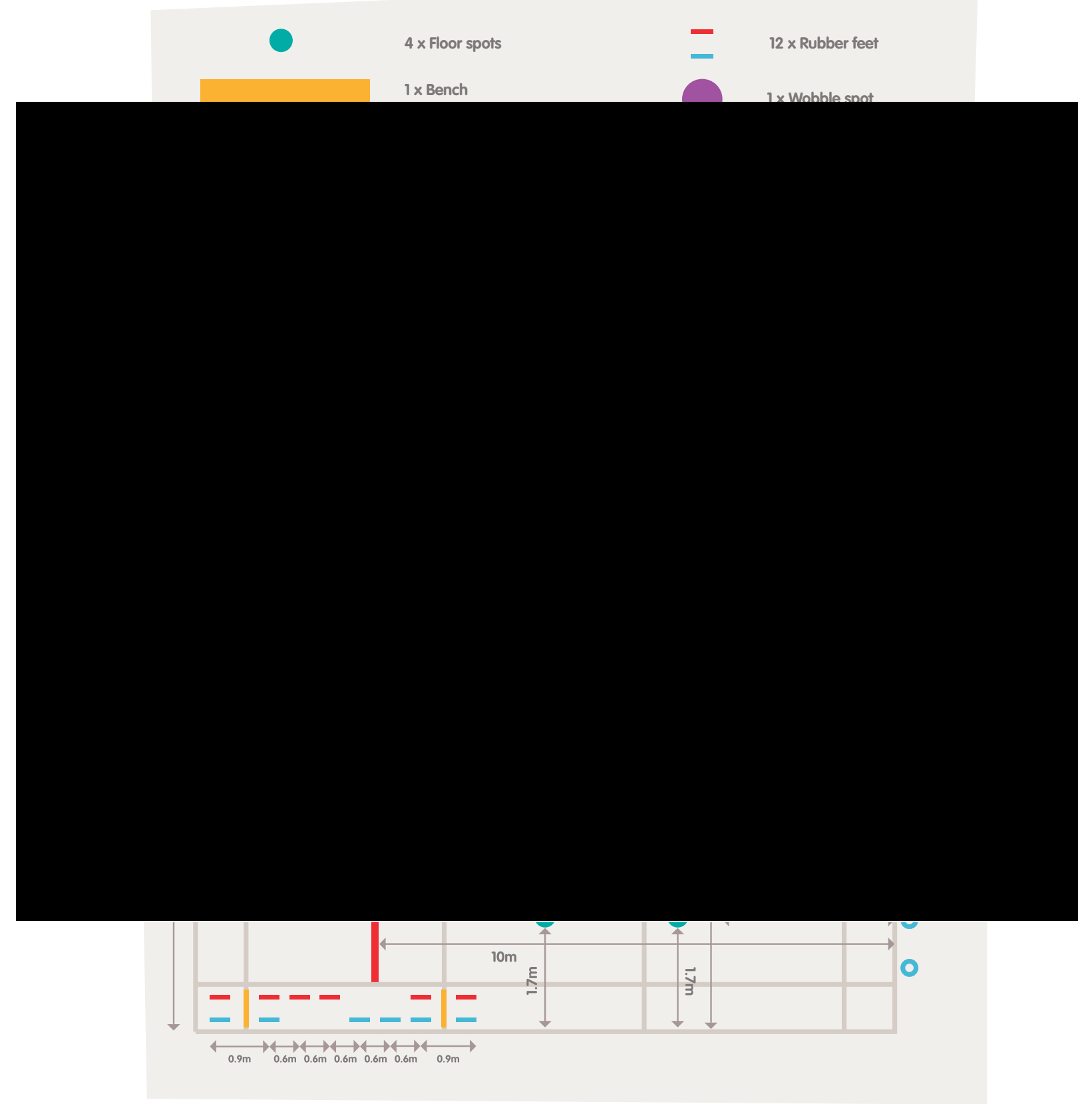




\section{군 Dragon Challenge}

This section provides directions for the administration of the Dragon Challenge. It is important that the Dragon Challenge is administered as specified so that a valid interpretation of a child's physical competence can be

achieved and the results can be assessed across time. Therefore administrators should be trained in the live

administration of Dragon Challenge. A range of video resources are available to support administrator training and development (see Appendix 3)

Let's run through the activities so you know what to do!" IMove onto Step 3 - Demonstrations,

Box 1. Script for introducing the Dragon Challenge to children 


\section{Step 3 - Demonstration of Dragon Challenge}

After the introduction, children then receive a practical demonstration of the Dragon Challenge activities. An assessor, administrator or specially recruited individual can demonstrate the activities. It is important that demonstrators perform each task accurately with sound technique. Demonstrations are important in ensuring that children understand the task requirements. However, they should not be used to teach or coach children

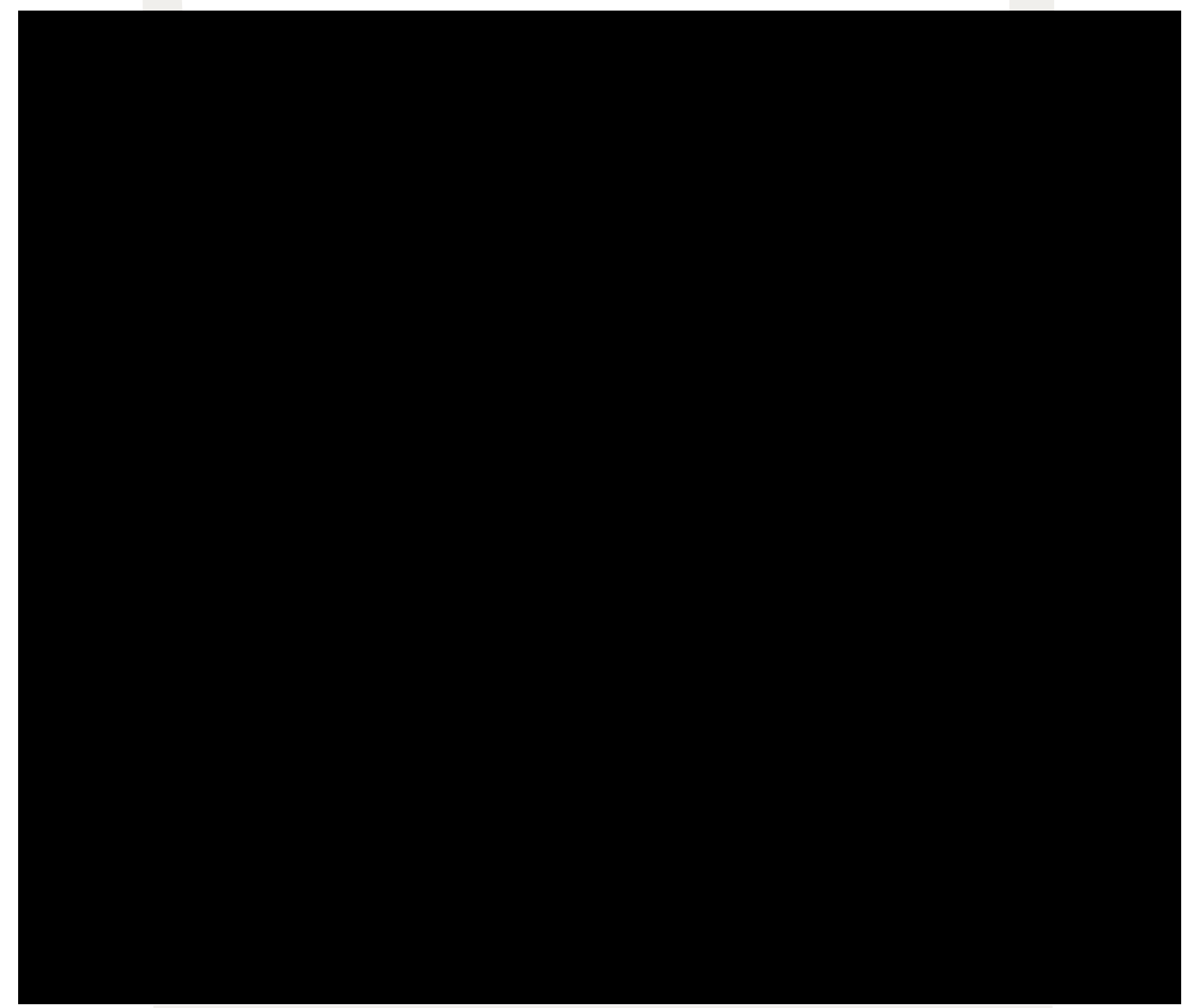

Demonstrate arch only "Let's see it all again.... what do you notice about their shoulders? Did they touch the floor?" [answer: off the floor throughout]

Repeat in full Questions to check understanding:

Return to iPad _ "What order do you do the shapes in?" [answer: dish-arch-dish-arch]

"Show me a dish? Show me an arch?" *children perform shapes*

"Do you start on your front or back?" [back] 


\begin{tabular}{|c|c|c|}
\hline Ectivity & Actions & Scripts \\
\hline Wobble Spot & $\begin{array}{l}\text { Run to wobble spot. } \\
\text { Pick up bean bag. Get } \\
\text { set. } \\
\text { Completes } 5 \text { bean bag }\end{array}$ & $\begin{array}{l}\text { "This is called wobble spot. You need to balance on } 1 \text { foot, } \\
\text { after picking up the bean bag." } \\
\text { "Get yourself set, what do I mean by set?" } \\
\text { [answer: balanced before starting] }\end{array}$ \\
\hline
\end{tabular}

bounce)

Questions to check understanding:

Return to iPad "What type of throw do you use?" [answer: underarm

Return to iPad $\quad$ "What type of throw do you use?" [answer: unde can you go?" [as close as you like]

"What do you do if you drop the ball or miss the catch?"

[return to iPad] 
$\begin{array}{ll}\text { T-agility } & \begin{array}{l}\text { Moves at half speed } \\ \text { through all points of 'T' } \\ \text { facing forwards (must } \\ \text { enter both right \& left } \\ \text { court tramlines). }\end{array} \quad \begin{array}{l}\text { "You start moving forwards and then side-step into the tramline } \\ \text { on the left then the tramline on the right." }\end{array} \\ \text { "You will always face forward and you need to move and change }\end{array}$

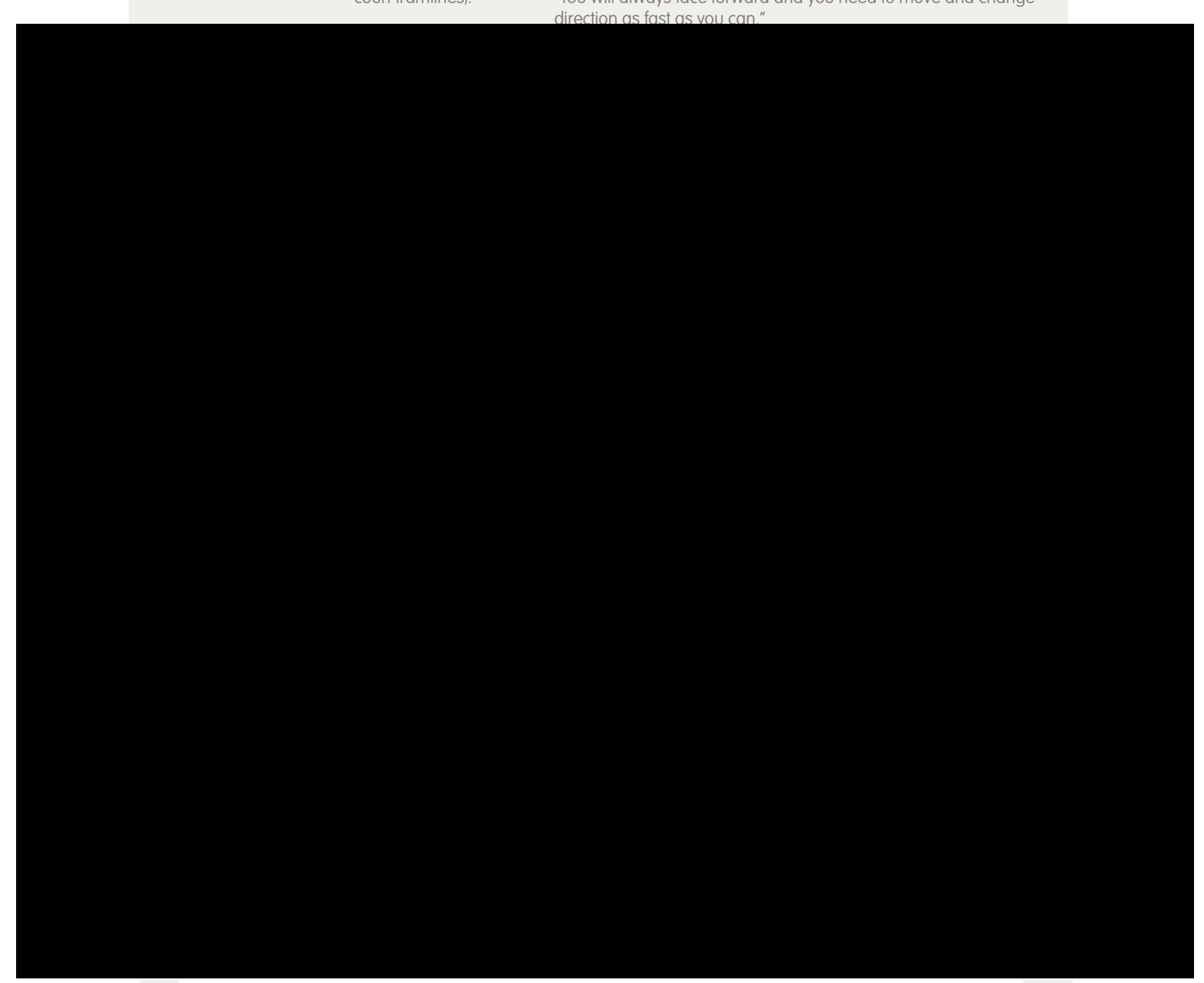

pear to understand the task, one additional demonstration can be given. No coaching tips or technical guidance should be given during practice opportunities. 


\section{Step 5 - During the Assessment}

While children do not have the opportunity to practice the full Dragon Challenge from start to finish, they should be provided with two practice trials for each individual task prior to assessment to assure that the child understands what to do. Following the demonstrations, children can be split into small groups, rotating around the activity stations to provide an opportunity for practice. When the child does not appear

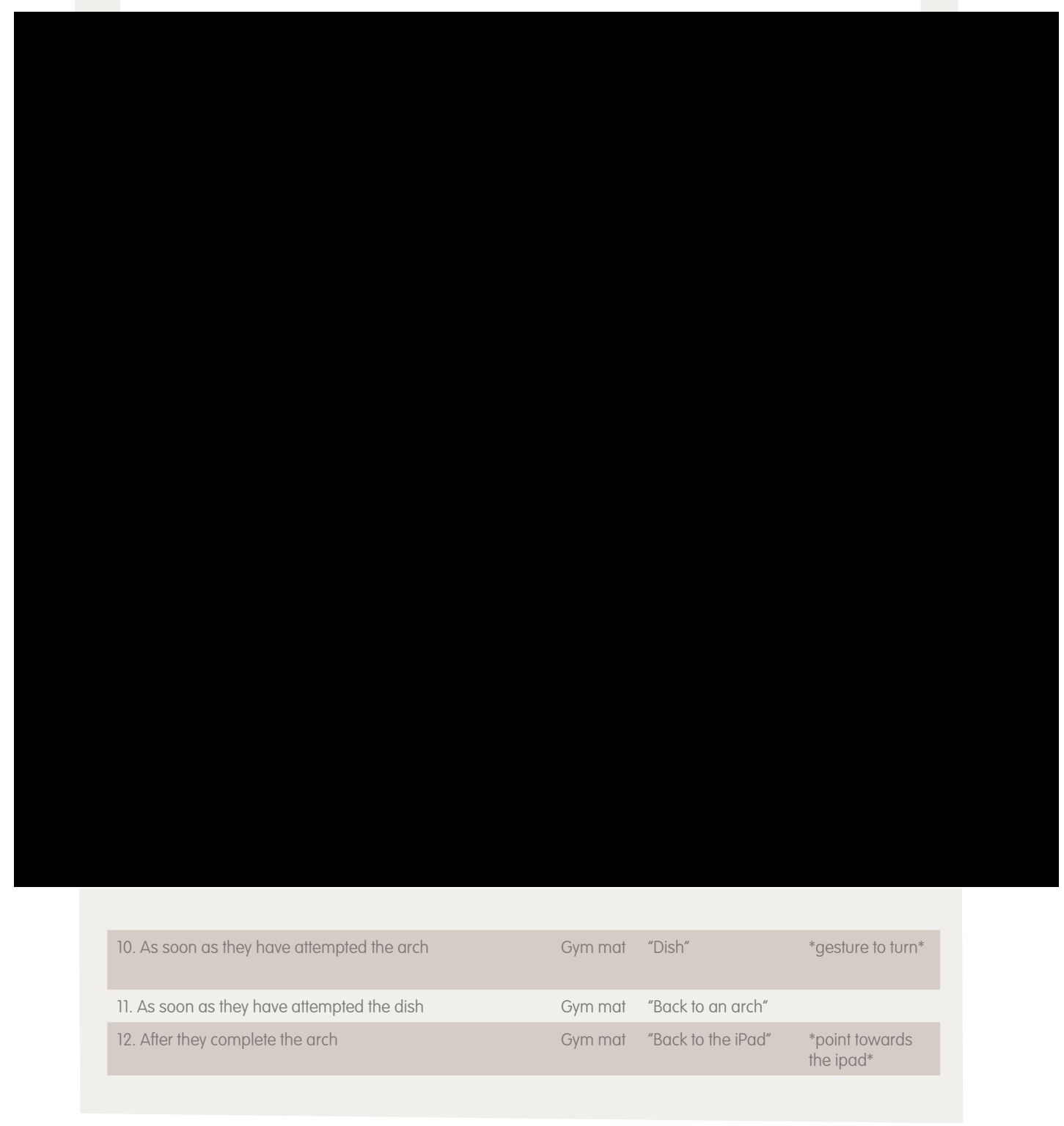




\begin{tabular}{|c|c|c|}
\hline 13. As soon as they touch the iPad & iPad & "Wobble spot" \\
\hline 14. As they approach the wobble spot & $\begin{array}{l}\text { Wobble } \\
\text { spot }\end{array}$ & $\begin{array}{l}\text { "Pick up the bean bag } \\
\text { and get yourself set" }\end{array}$ \\
\hline 15. As soon as they are set on the wobble & Wobble & "Go-1, 2, 3, 4, 5" \\
\hline
\end{tabular}

spot

spot

\begin{tabular}{|c|c|c|c|}
\hline 33. As they approach the cone gate & Sprint & "As fast as you can" & \\
\hline 34. Once they cross over the finish line & Sprint & "Well done, great effort!" & $\begin{array}{l}\text { *clap hands, high five } \\
\text { child* }\end{array}$ \\
\hline
\end{tabular}


Table 6 Additional directions for the administrator

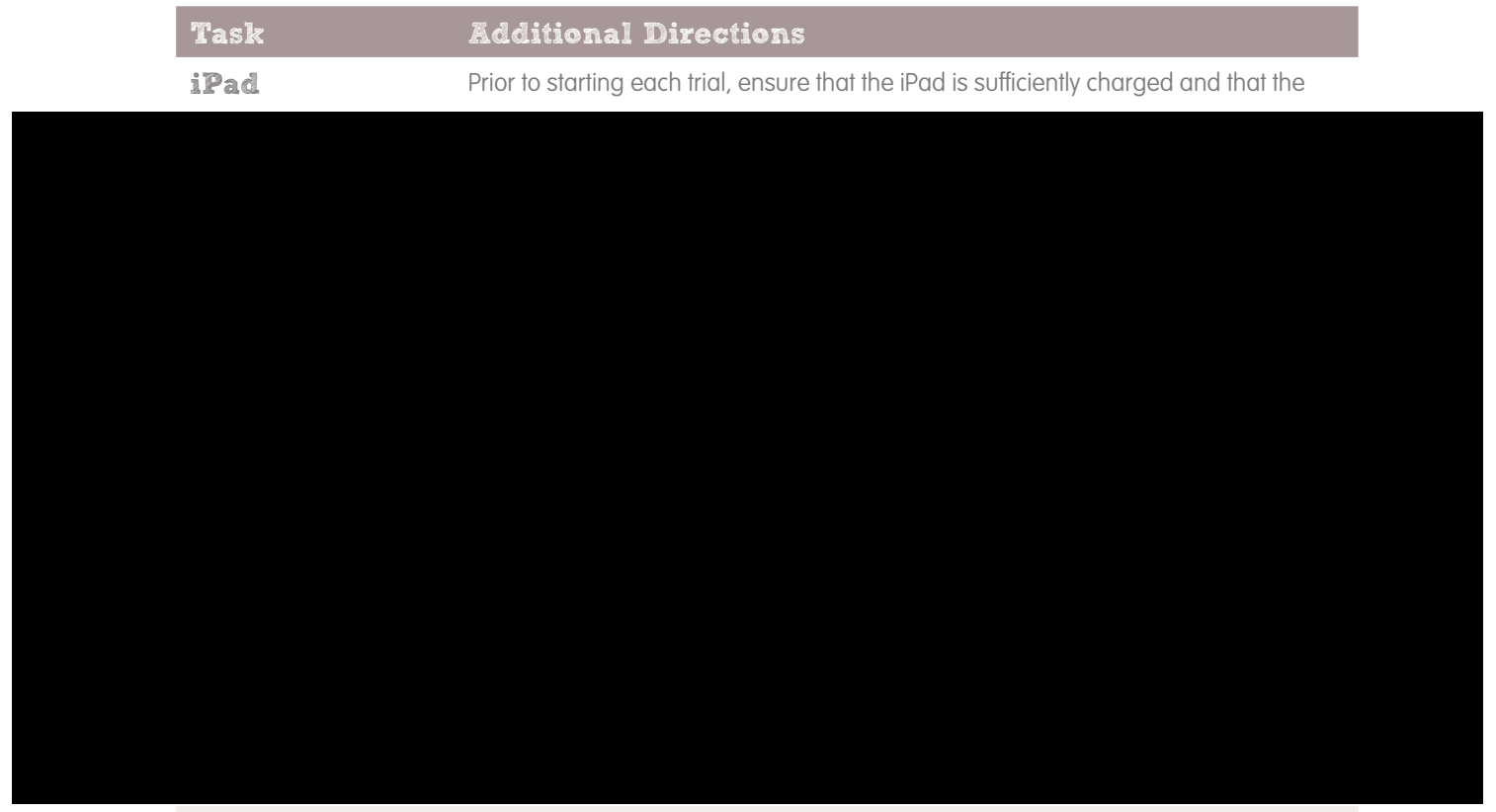

Patterns

assessors by running alongside the jumping pattern 


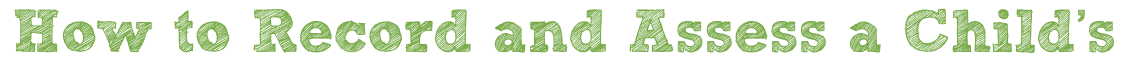
Dragon Challenge Peritormance

This section provides information in relation to how to record and assess a child's performance at the Dragon Challenge. As noted on page 13, all assessors of Dragon Challenge must have received training in assessment and be confident and competent in assessing children. 
Assessment Criteria

Child performance at Dragon Challenge is assessed through

- Time to completion

- Skill performance criteria (technique and outcomel 
Table 7 Dragon Challenge assessment performance criteria

\begin{tabular}{llll} 
DC Task & Technical Criterion & Technical Criterion & Outcome Criterion \\
\hline $\begin{array}{ll}\text { 1. Balance Bench } \\
\text { 1.1 Moves without hesitation } \\
\text { up to turn }\end{array}$ & $\begin{array}{l}1.2 \text { Body posture stable } \\
\text { (head \& trunk stable, } \\
\text { minimal arm flailing) }\end{array}$ & $\begin{array}{l}1.3 \text { Walks length of beam, } \\
\text { completes full turn at 3/4 mark } \\
\text { without falling off, dismounts at } \\
\text { end zone }\end{array}$ \\
\end{tabular}

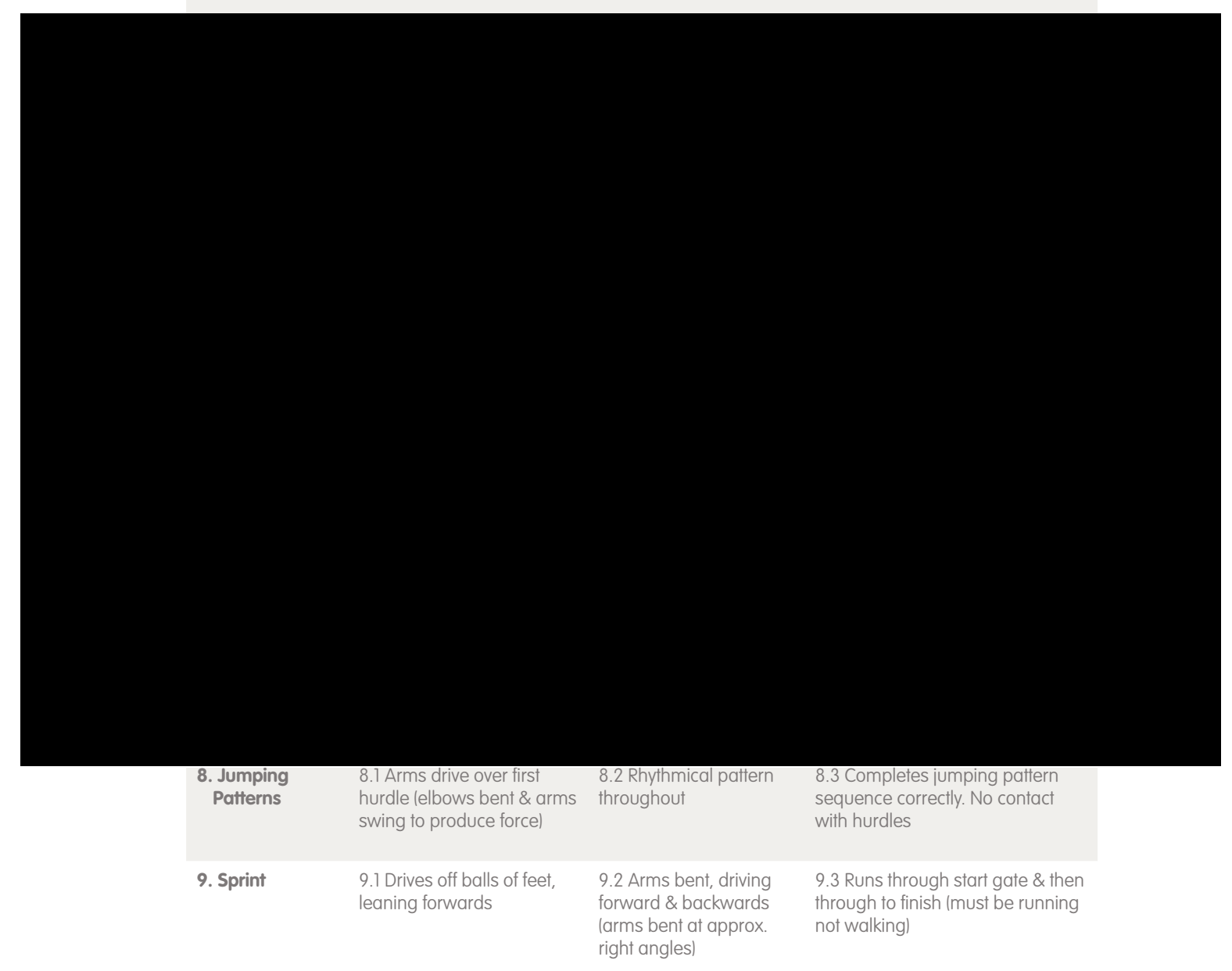




\section{Calculating the Dragon Challenge Score}

The Dragon Challenge composite score is calculated from the same trial as follows: 
Table 8 Scoring the Technique and Outcome Skill Performance Criteria

\begin{tabular}{|c|c|c|c|c|c|c|}
\hline \multirow{2}{*}{$57 x$} & \multicolumn{3}{|c|}{ Technique (Process) } & \multicolumn{3}{|c|}{ Outcome (Product) } \\
\hline & $\begin{array}{c}\text { DC Criteria } \\
\#\end{array}$ & $\begin{array}{l}\text { Points per } \\
\text { criteria }\end{array}$ & $\begin{array}{l}\text { Scoring } \\
\text { range }\end{array}$ & $\begin{array}{c}\text { DC Criteria } \\
\#\end{array}$ & $\begin{array}{r}\text { Points per } \\
\text { Criteria }\end{array}$ & Scoring \\
\hline Balance Bench & $1.1,1.2$ & 1 & $0-2$ & 1.3 & 2 & 0,2 \\
\hline
\end{tabular}

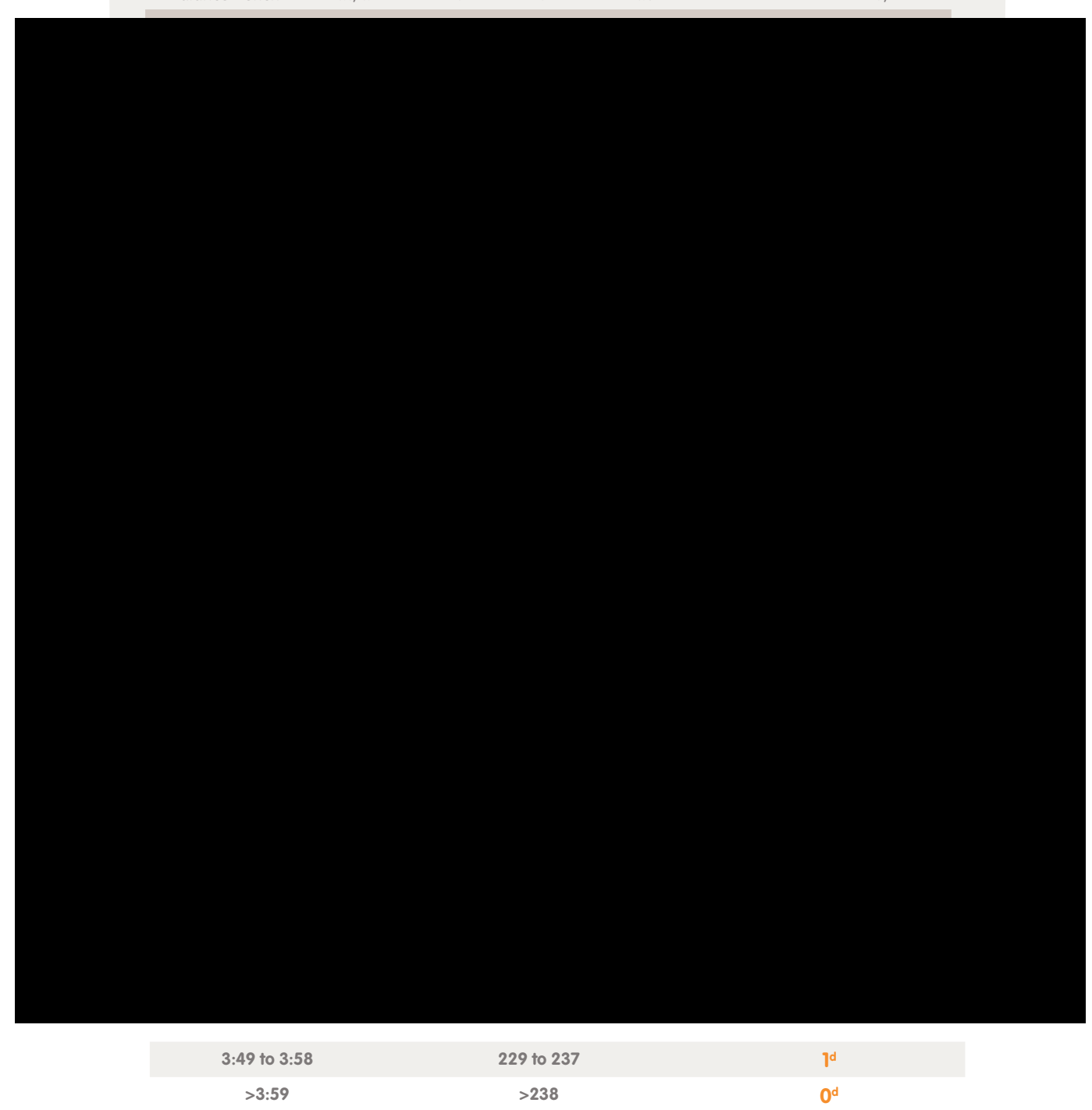


25

\section{Interpreting the \\ Dragon Challenge ?esults}

Interpreting the Dragon Challenge Total

A child's Dragon Challenge total score can be interpreted by examining their performance in relation to

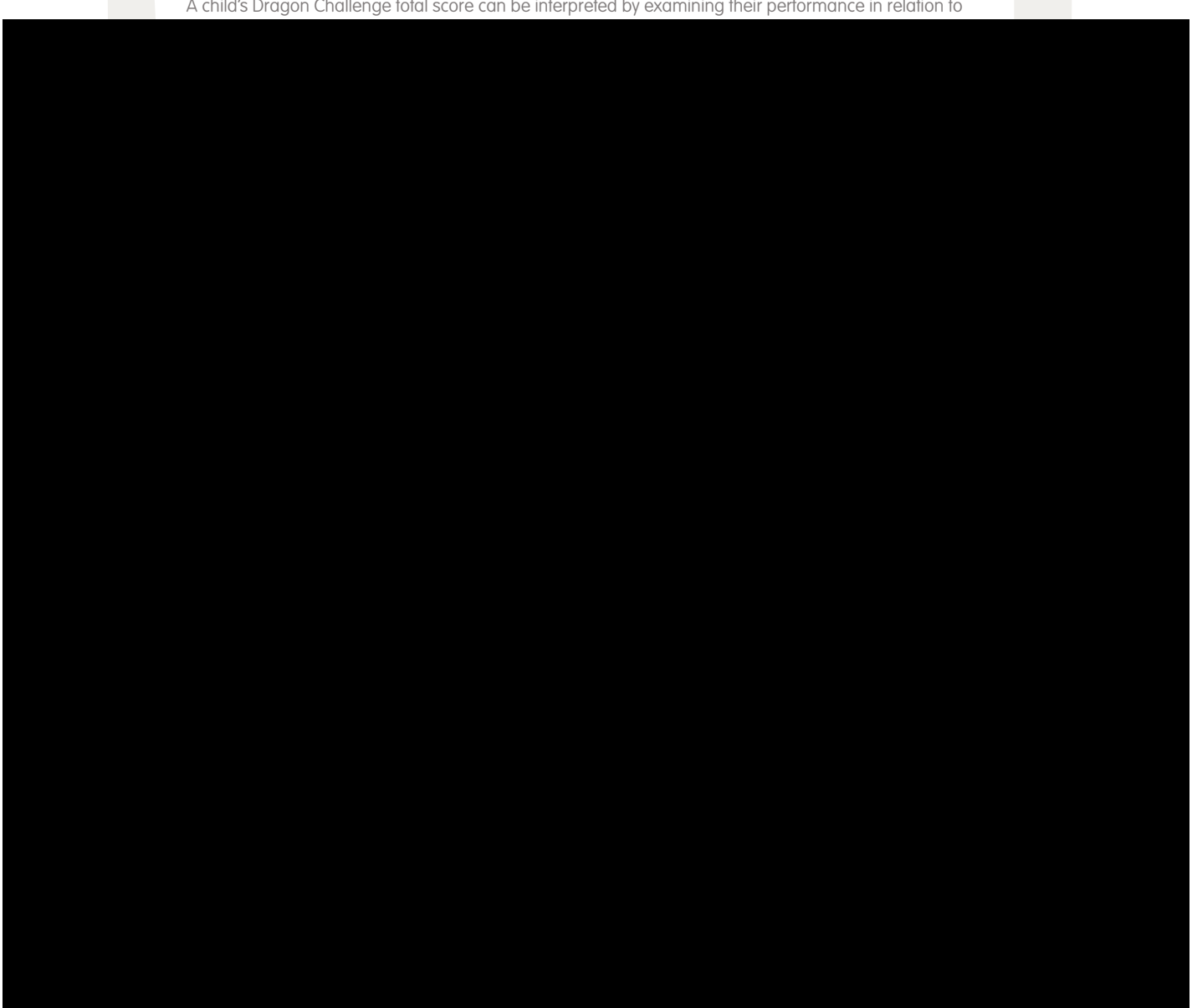

different sports and activities to advance and

encouraged to keep up the fantastic work

maintain their physical skills

*based on pilot data $(n=584)$ collected across Wales in Spring/Summer 2015 


\section{Interpreting Time Scores}

Judgements surrounding the speed in which a child completes the Dragon Challenge can be made by

drawing comparisons to other children. As shown in Table 10, each time score is assigned a bronze, silver,

gold or platinum ranking. The classification boundaries are at the 33rd, 66th and 95th percentile, consisted

with those used for interpreting the overall score. 


\section{Data Management}

This section discusses important matters concerning the recording and collation of Dragon Challenge results. Data management needs to be accurate and timely, and requires careful attention. The collation of results is important for subsequent analysis and monitoring, enabling detailed profiles of children's performances
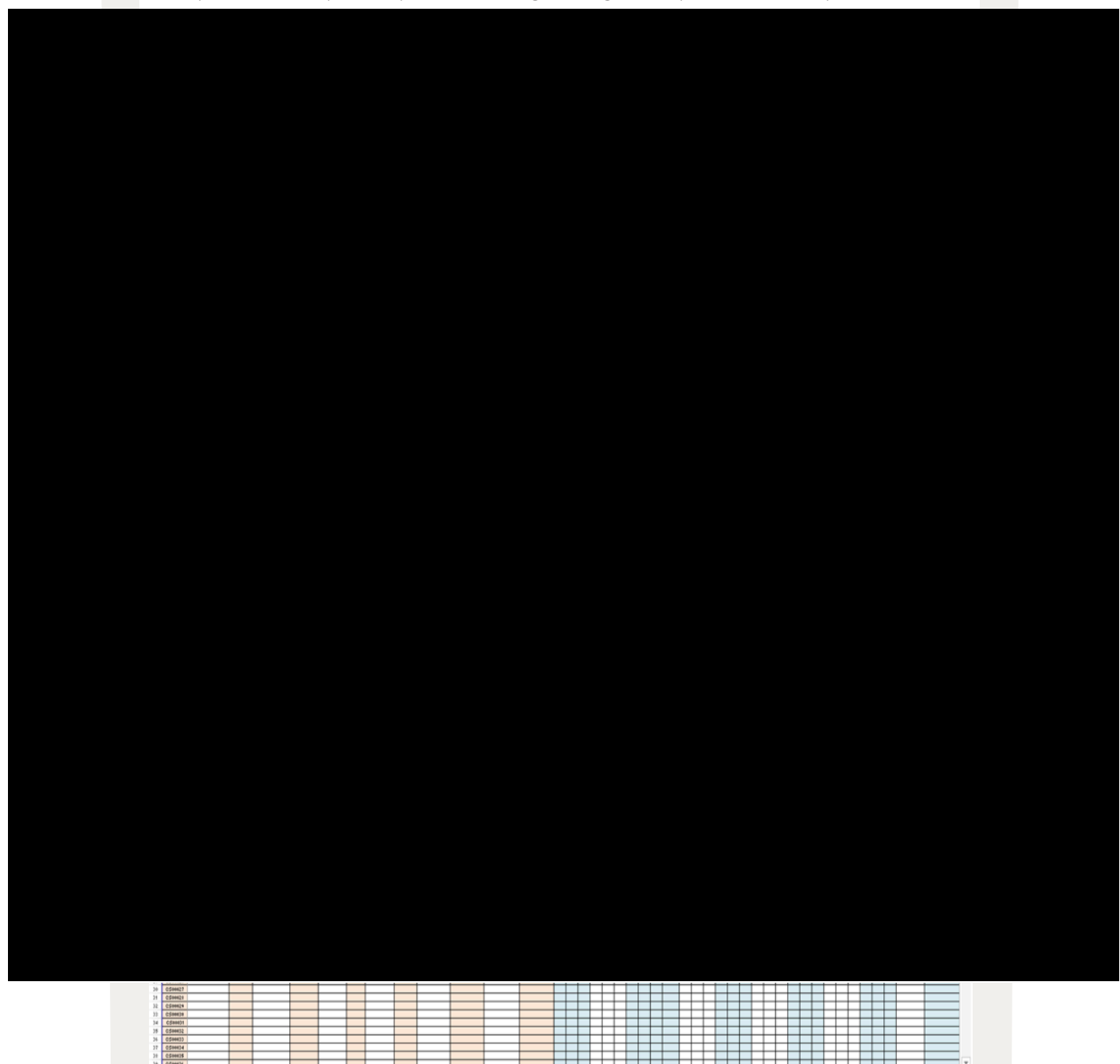

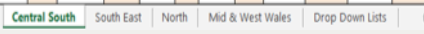


Table 11 Main data sheet: Explanation of variables and coding

\begin{tabular}{clc} 
Variable & \multicolumn{1}{c}{ Input } & Missing data \\
\hline Student No. & $\begin{array}{l}\text { A 7-character unique child identifier } \\
\text { number (page x) }\end{array}$ & $\mathrm{n} / \mathrm{a}$ \\
\hline School & Type school name in full & 99999 \\
\hline School year* & "YEAR 6" or "YEAR 7" & 99999
\end{tabular}

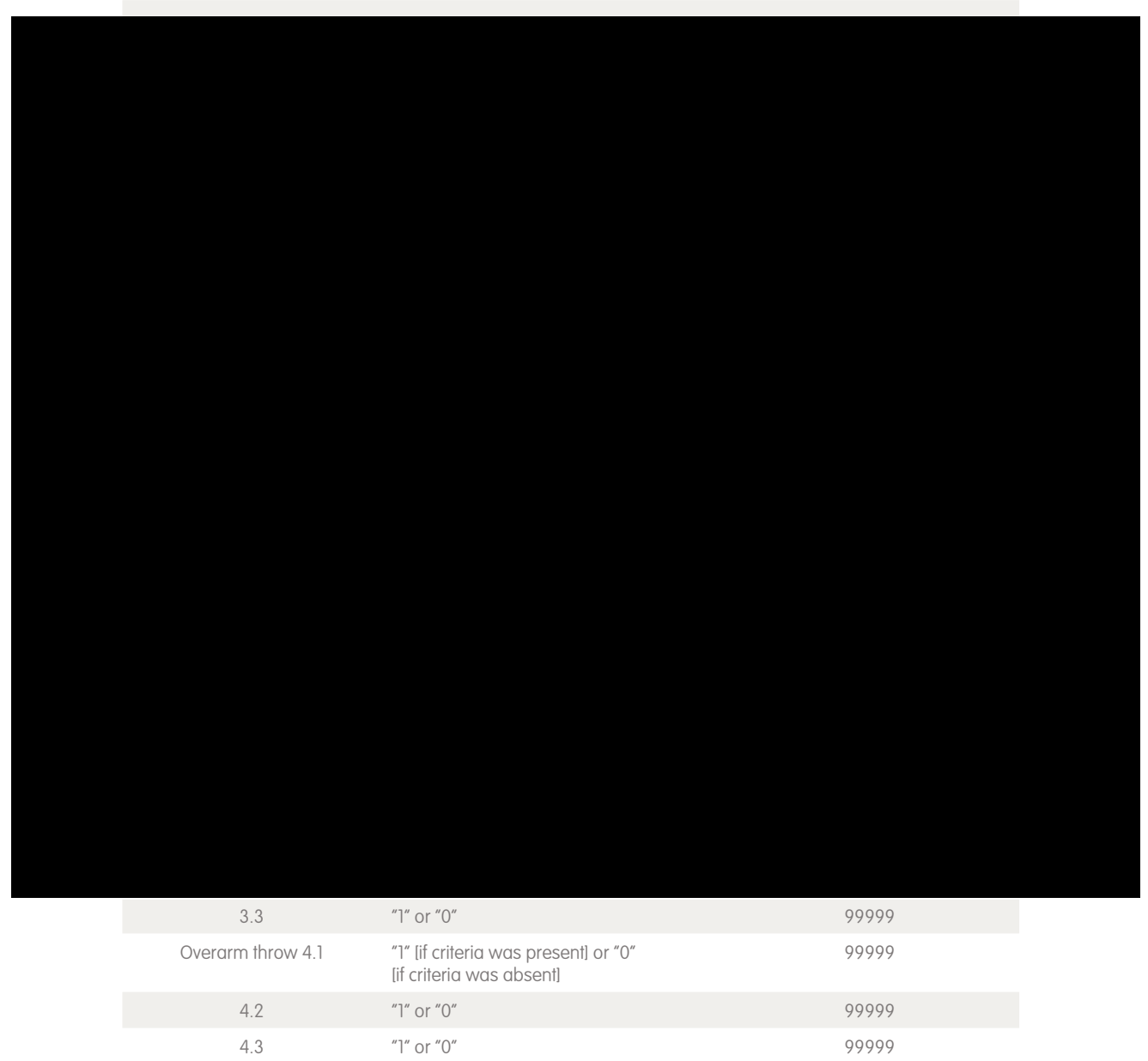


29

$\begin{array}{clr}\text { Basketball dribble 5.1 } & \text { "1" lif criteria was present] or "0" } & 99999 \\ & \text { [if criteria was absent] } & 99999 \\ 5.2 & \text { "1" or "0" } & 99999 \\ 5.3 & \text { "1" or "0" } & 99999 \\ \text { Catch 6.1 } & \text { "1" [if criteria was present] or "0" } & \end{array}$

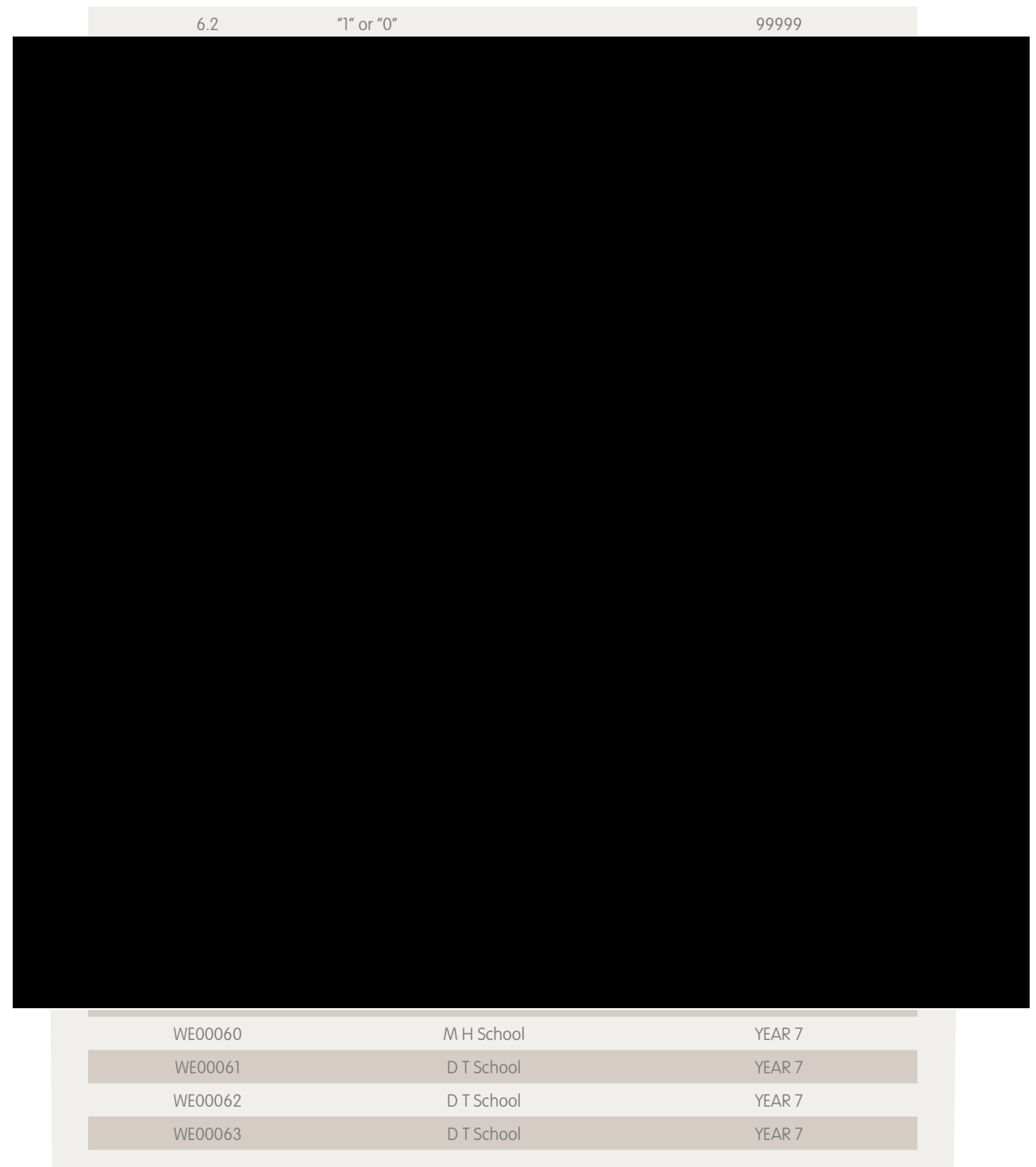




\section{Unique Identifier Number: Assessors}

After successfully completing training, each assessor is given a unique identifier number by the regional lead Gold Standard Assessor. This number is validated against a regional database of assessors to avoid duplication. The coding helps identify the assessor's region, gender and professional role. The method for generating a unique identifier number for assessors is shown in Table 13 below:

One out of every 10 children should have a repeat assessment. This can occur through sharing video data of a child performance with a gold assessor (after obtaining the necessary permissions) or through paired observations with a gold assessor during in situ live assessments. All quality assurance checks should be documented by the lead regional gold assessor and appropriate re-training given if required. 


\section{REFERENCES}

Babic, M.J., Morgan P. J., Plotnikoff, R.C. Lonsdale, C. White, R.L \& Lubans, D.R. (2014). Physical activity and physical self-concept in youth: systematic review and meta-analysis. Sports Medicine, 44(11): 1589-601.

Booth, J.N., Leary, S.D., Joinson, C., Ness, A.R., Tomporowski, P.D., Boyle, J.M., \& Reilly, J.J. (2014). Associations between objectively measured physical activity and academic attainment in adolescents from a UK cohort. British Journal of Sports Medicine, 48(3): 265-70

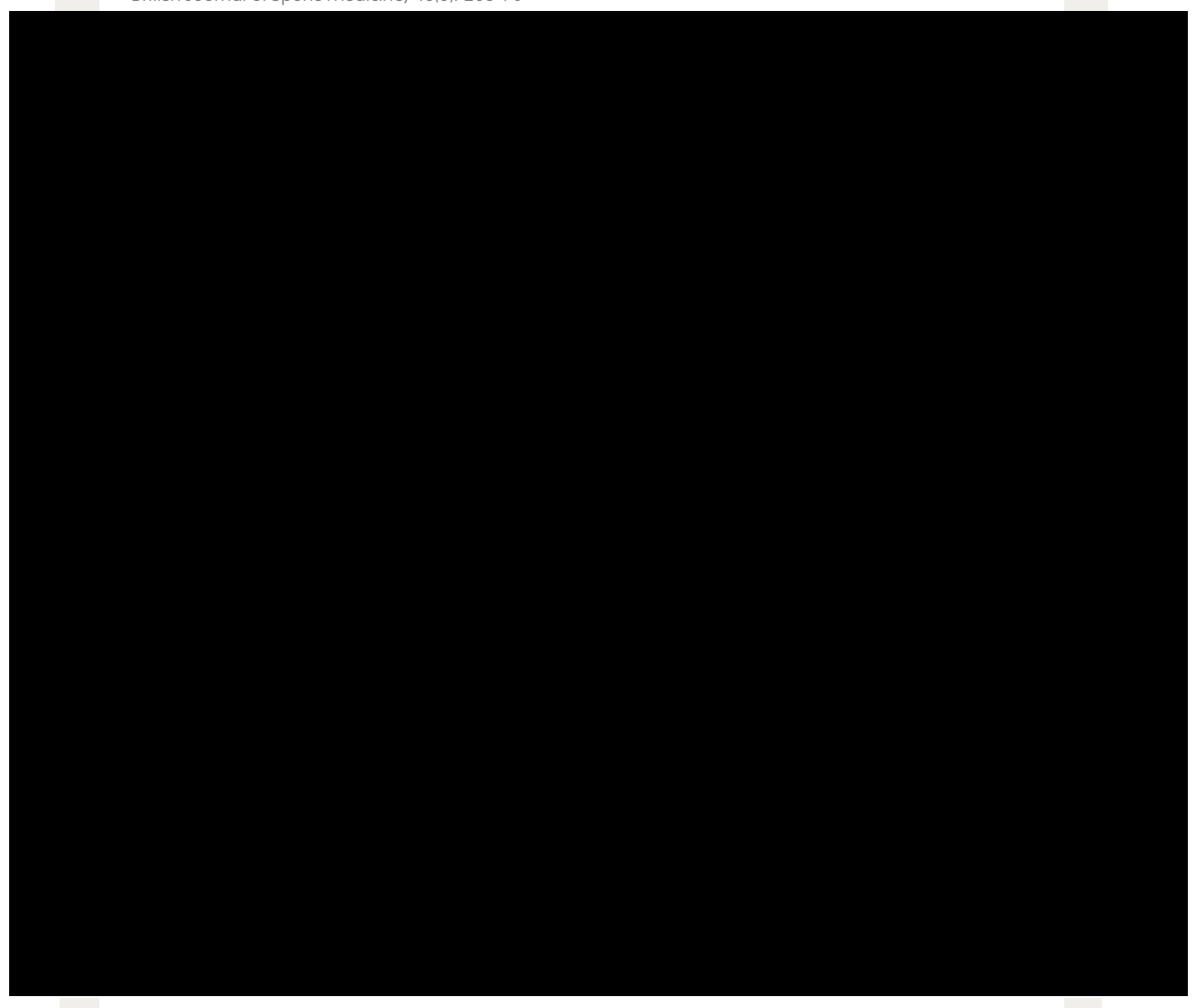

Vlahov, E., Baghurst, T. M., \& Mwavita, M. (2014). Preschool motor development predicting high school health-related physical fitness: a prospective study. Perceptual and Motor Skills, 119(1), 279-291. doi: 10.2466/10.25.PMS.119c16z8

Whitehead, M. (2001). The concept of physical literacy. European Journal of Physical Education, 6(2): 127-138. 


\section{APPENDICES}

APPENDIX A - LIST OF VIDEO RESOURCES

Video Material to Support Delivery of Dragon Challenge V1.0 


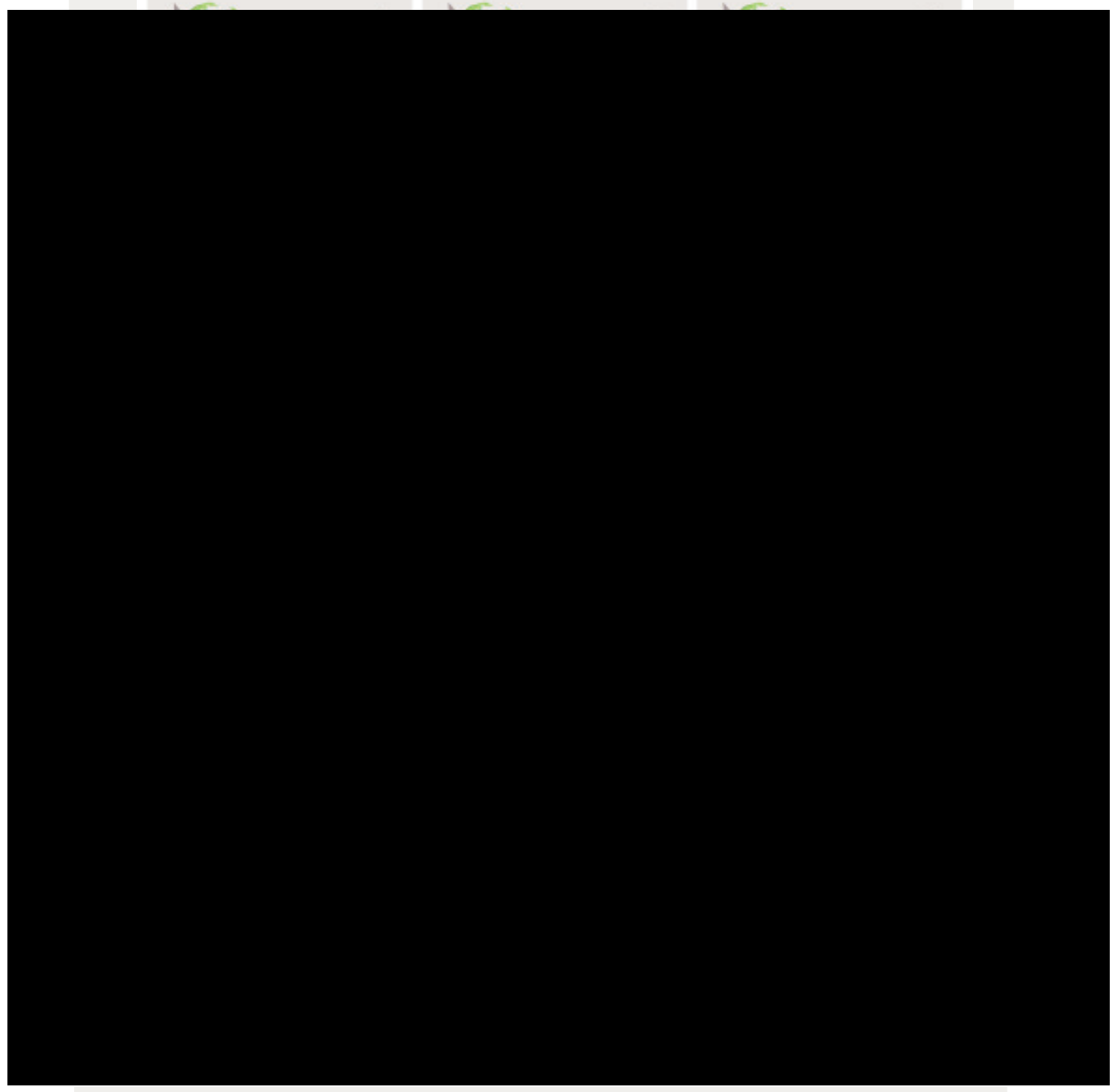


APPENDIX E - INFORMATION FOR PROFESSIONALS AND PARENTS/CARERS

Information for professionals (teachers/teaching assistants, Sports Development, Sport Ambassadors, coaches, dance teaches, etc.

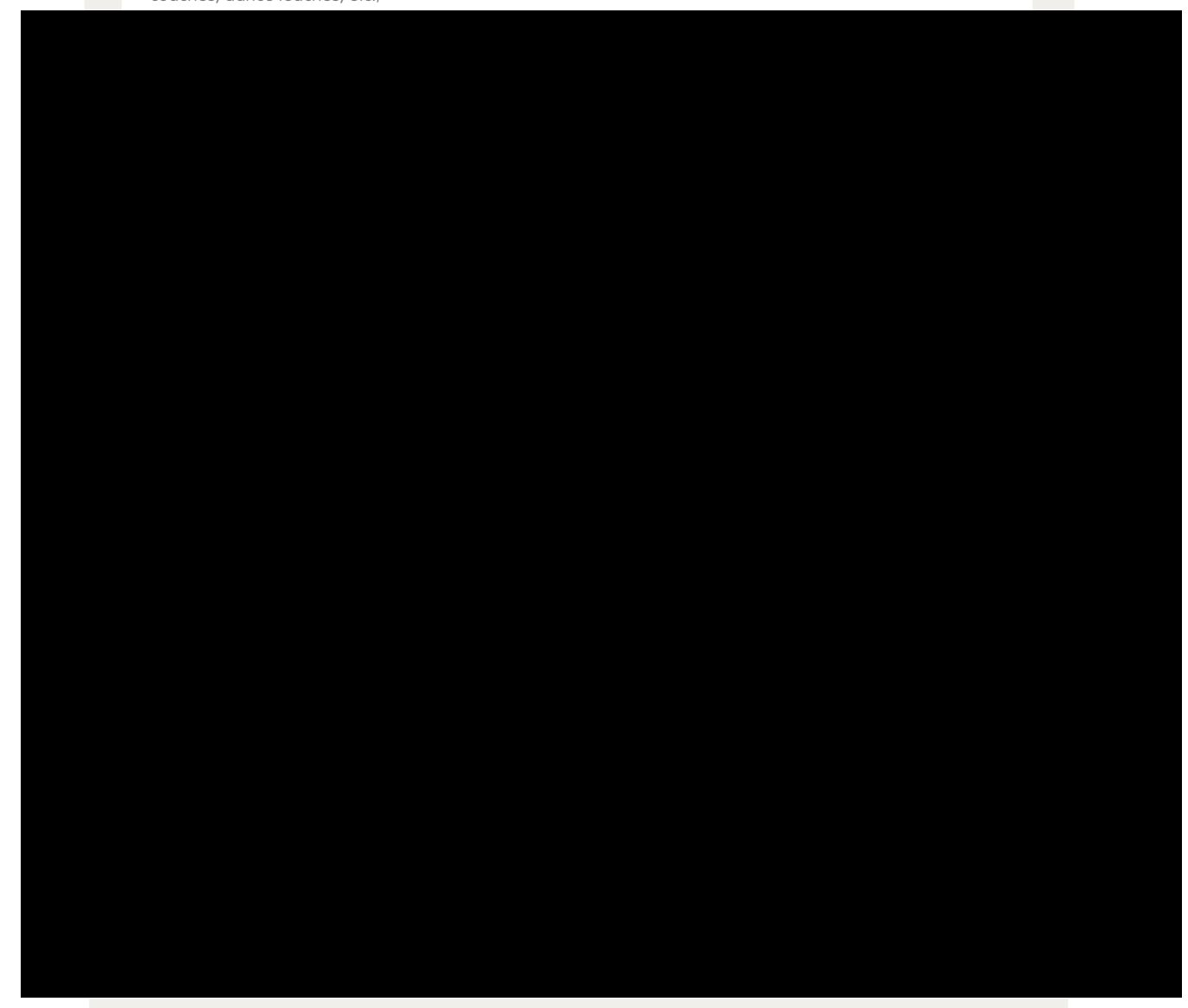




\section{Appendix VI: Dragon Challenge Video Resources}

Video material to support delivery of the Dragon Challenge:

Video 1.

Title: Dragon Challenge Circuit

Hyperlink: https://www.youtube.com/watch?v=1SPLtwDrgRM

Video 2.

Title: Dragon Challenge Setup \& Equipment

Hyperlink: https://www.youtube.com/watch?v=bg8QUxs78qo

Video 3.

Title: Dragon Challenge Introduction and Demonstration

Hyperlink: https://www.youtube.com/watch?v=3Pg_IHyAGxE

Video 4.

Title: Dragon Challenge Promotional video for Kids English

Hyperlink: https://www.youtube.com/watch?v=Ddr17NbqV5I

Video 5 .

Title: Dragon Challenge Promotional video for Kids Welsh

Hyperlink: https://www.youtube.com/watch?v=DEs2kyLiaok

Video 6.

Title: What is the Dragon Challenge?

Hyperlink: https://www.youtube.com/watch?v=9XD-ejbfaE8

Video 7.

Title: Dragon Challenge Promotional video for Administrators \& Assessors

Hyperlink: https://www.youtube.com/watch?v=POq5cu0PWGQ

Video 8.

Title: Children's Guide to the Dragon Challenge

Hyperlink: https://www.youtube.com/watch?v=ebLNCS7DTv0 


\section{Appendix VII: Children's Perceived Benefits/Barriers to Exercise Questionnaire}

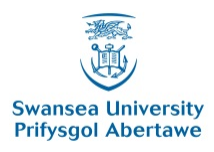

Applied Sports Technology Exercise and Medicine Research Centre (A-STEM)

Sport and Health Portfolio, College of Engineering

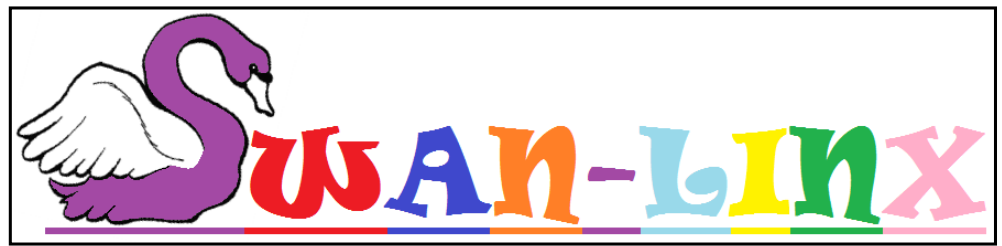

\section{Children's Perceived Benefits/Barriers to Exercise Questionnaire}

(Garcia et al., 1995)

ID Number: (Fitness Fun Day Number)

Are you a: (please circle one)

Boy

Girl

How old are you:

years old

In this programme, when we talk about physical activity, we mean any movement that requires your body to work harder than it does whilst sitting, or resting. Physical activity is any activity that makes your heart beat faster or makes you get out of breath some of the time.

Why are we asking you to do this questionnaire? We want to know what YOU think about physical activity, sports and exercise.

\section{Please Remember:}

- There are no right or wrong answers. We only want to know what you think, so please answer truthfully.

- If you do not know an answer, please write your best guess.

- There is no time limit, so please take time reading each question carefully. 
1. Children say there are many different reasons that they like to be active or play sports or dance. Being active is anything that you do when you are moving, exercising or not sitting still. Below are some reasons that other children have told us why they like to be active. For each reason, tell us what you think. If you think it is a good reason then you would "Agree a little" or "Agree a lot". If you do not think it's a good reason, then you would "Disagree a little" or "Disagree a lot". If you are not sure or you don't think the reason is good or bad then you are "in between".

(Please circle one number for each reason)

\begin{tabular}{|l|c|c|c|c|c|}
\hline $\begin{array}{c}\text { A reason that I might be } \\
\text { active is because when I } \\
\text { am active }\end{array}$ & $\begin{array}{c}\text { Disagree } \\
\text { a lot }\end{array}$ & $\begin{array}{c}\text { Disagree } \\
\text { a little }\end{array}$ & $\begin{array}{c}\text { In } \\
\text { between }\end{array}$ & $\begin{array}{c}\text { Agree } \\
\text { a little }\end{array}$ & $\begin{array}{c}\text { Agree } \\
\text { a lot }\end{array}$ \\
\hline$\ldots$.I look better & 1 & 2 & 3 & 4 & 5 \\
\hline$\ldots$ I have more energy & 1 & 2 & 3 & 4 & 5 \\
\hline$\ldots$ I feel happier & 1 & 2 & 3 & 4 & 5 \\
\hline$\ldots$.I have fun & 1 & 2 & 3 & 4 & 5 \\
\hline$\ldots$ I make more friends & 1 & 2 & 3 & 4 & 5 \\
\hline$\ldots$ I get stronger & 1 & 2 & 3 & 4 & 5 \\
\hline$\ldots$ I like myself more & 1 & 2 & 3 & 4 & 5 \\
\hline$\ldots$. get in better shape & 1 & 2 & 3 & 4 & 5 \\
\hline$\ldots$ I feel healthier & 1 & 2 & 3 & 4 & 5 \\
\hline
\end{tabular}


2. Children say there are also reasons that make it hard for them to be active. For each reason, tell us what you think. If you think it is a good reason then you would "Agree a little" or "Agree a lot". If you do not think it's a good reason, then you would "Disagree a little" or "Disagree a lot". If you are not sure or you don't think the reason is good or bad then you are "in between".

(Please circle one number for each reason)

\begin{tabular}{|l|c|c|c|c|c|}
\hline I might not be active if... & $\begin{array}{c}\text { Disagree } \\
\text { a lot }\end{array}$ & $\begin{array}{c}\text { Disagree } \\
\text { a little }\end{array}$ & $\begin{array}{c}\text { In } \\
\text { between }\end{array}$ & $\begin{array}{c}\text { Agree } \\
\text { a little }\end{array}$ & $\begin{array}{c}\text { Agree } \\
\text { a lot }\end{array}$ \\
\hline $\begin{array}{l}\ldots \text { I didn't have enough } \\
\text { time to be active }\end{array}$ & 1 & 2 & 3 & 4 & 5 \\
\hline $\begin{array}{l}\ldots \text { I have too many } \\
\text { chores to do }\end{array}$ & 1 & 2 & 3 & 4 & 5 \\
\hline $\begin{array}{l}\ldots \text { I didn't have a good } \\
\text { place to be active }\end{array}$ & 1 & 2 & 3 & 4 & 5 \\
\hline $\begin{array}{l}\ldots \text { If the weather was too } \\
\text { bad }\end{array}$ & 1 & 2 & 3 & 4 & 5 \\
\hline $\begin{array}{l}\ldots \text { I didn't have the right } \\
\text { clothes/shoes }\end{array}$ & 1 & 2 & 3 & 4 & 5 \\
\hline $\begin{array}{l}\ldots \text { I didn't know how to } \\
\text { do the activity }\end{array}$ & 1 & 2 & 3 & 4 & 5 \\
\hline $\begin{array}{l}\ldots \text { I didn't have the right } \\
\text { equipment }\end{array}$ & 1 & 2 & 3 & 4 & 5 \\
\hline $\begin{array}{l}\ldots \text { I had too much } \\
\text { homework }\end{array}$ & 1 & 2 & 3 & 4 & 5 \\
\hline $\begin{array}{l}\ldots . \text { I didn't have anyone } \\
\text { to be active with }\end{array}$ & 1 & 2 & 3 & 4 & 5 \\
\hline $\begin{array}{l}\ldots \text { I didn't like to be } \\
\text { active }\end{array}$ & 1 & 2 & 3 & 4 & 5 \\
\hline
\end{tabular}




\section{Appendix VIII: Children's Self-perceptions of Adequacy in and Predilection for Physical Activity Questionnaire}

Applied Sports Technology Exercise and Medicine Research Centre (A-STEM)

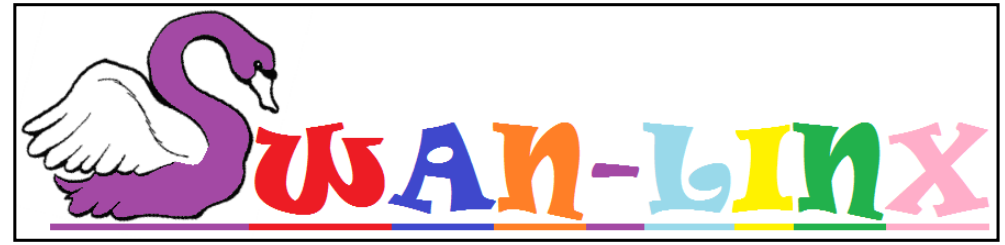

\section{What's Most Like Me?}

\section{Questionnaire}

(Hay, 1992)

ID Number: (Fitness Fun Day Number)

Are you a: (please circle one)

Boy

Girl

How old are you:

years old

In this programme, when we talk about physical activity, we mean any movement that requires your body to work harder than it does whilst sitting, or resting. Physical activity is any activity that makes your heart beat faster or makes you get out of breath some of the time.

Why are we asking you to do this questionnaire? We want to know what YOU think about physical activity, sports and exercise.

\section{Please Remember:}

- There are no right or wrong answers. We only want to know what you think, so please answer truthfully.

- If you do not know an answer, please write your best guess.

- There is no time limit, so please take time reading each question carefully. 
For the questions below you have to read 2 sentences and the circle the sentence you think is MORE LIKE YOU.

For example try the following question:

Some kids have one nose on their face! BUT Other kids have three noses on their face!

That shouldn't be too hard for you to decide! Once you have circled the sentence that is more like you, then you have to decide if it is REALLY TRUE for you or SORT OF TRUE for you.

Here is another sample question for you to try. Remember, first circle the sentence that is more like you and then put a check $(\checkmark)$ in the correct box if it is really true or only sort of true for you.

REMEMBER THERE ARE NO RIGHT OR WRONG ANSWERS, JUST WHAT IS MOST LIKE YOU.

\begin{tabular}{|c|c|c|c|}
\hline Some kids like to play $\mathrm{n}$ & th computers & BUT & Other kids don't like playing with computers \\
\hline$\square$ REALLY TRUE for me & $\begin{array}{l}\square \text { SORT OF TRUE } \\
\text { for me }\end{array}$ & & $\begin{array}{cc}\square \text { REALLY TRUE for me } & \begin{array}{c}\text { SORT OF TRUE } \\
\text { for me }\end{array}\end{array}$ \\
\hline
\end{tabular}

Now you are ready to start filling in this form. Take your time and do the whole form carefully.

If you have any questions, just ask!

If you think you are ready you can start now.

\section{BE SURE TO FILL IN EACH PAGE!}




\begin{tabular}{|lcll|}
\hline $\begin{array}{l}\text { Some kids can't wait to play active games } \\
\text { after school }\end{array}$ & BUT & $\begin{array}{l}\text { Other kids would rather do something else } \\
\text { after school }\end{array}$ \\
$\begin{array}{l}\square \text { REALIYTRUE } \\
\text { for me }\end{array}$ & $\begin{array}{c}\text { SORT OF TRUE } \\
\text { for me }\end{array}$ & $\begin{array}{l}\square \text { REALY TRUE } \\
\text { for me }\end{array}$ & $\begin{array}{c}\square \text { SORT OF TRUE } \\
\text { for me }\end{array}$ \\
\hline
\end{tabular}

\begin{tabular}{|c|c|c|c|}
\hline Some kids don't & ying active games & BUT & Other kids really like playing active games \\
\hline $\begin{array}{l}\square \text { REALLY TRUE } \\
\text { for me }\end{array}$ & $\begin{array}{l}\text { 口 SORT OF TRUE } \\
\text { for me }\end{array}$ & & $\begin{array}{l}\text { Q SORT OF TRUE } \\
\text { for me }\end{array}$ \\
\hline
\end{tabular}

\begin{tabular}{|c|c|c|c|c|}
\hline \multicolumn{2}{|c|}{ ids don't have much fun playing sports } & BUT & Other kids have & time playing sports \\
\hline $\begin{array}{l}\square \text { REALLY TRUE } \\
\text { for me }\end{array}$ & $\begin{array}{l}\square \text { SORT OF TRUE } \\
\text { for me }\end{array}$ & & $\begin{array}{l}\square \text { REALIY TRUE } \\
\text { for me }\end{array}$ & $\begin{array}{l}\text { प SORT OF TRUE } \\
\text { for me }\end{array}$ \\
\hline
\end{tabular}

\begin{tabular}{|c|c|c|c|}
\hline Some kids are gc & ctive games & BUT & Other kids find active games hard to play \\
\hline $\begin{array}{l}\square \text { REALLYTRUE } \\
\text { for me }\end{array}$ & $\begin{array}{l}\square \text { SORT OF TRUE } \\
\text { for me }\end{array}$ & & $\begin{array}{l}\square \text { REALYY TRUE } \\
\text { for me }\end{array}$ \\
\hline
\end{tabular}

\begin{tabular}{|c|c|c|c|}
\hline Some kids don't & ying sports & BUT & Other kids really enjoy playing sports \\
\hline $\begin{array}{l}\square \text { REALLY TRUE } \\
\text { for me }\end{array}$ & $\begin{array}{l}\square \text { SORT OF TRUE } \\
\text { for me }\end{array}$ & & $\begin{array}{l}\square \text { SORT OF TRUE } \\
\text { for me }\end{array}$ \\
\hline
\end{tabular}

\begin{tabular}{|c|c|c|c|c|}
\hline \multicolumn{2}{|c|}{$\begin{array}{l}\text { Some kids always hurt themselves when they } \\
\text { play sports }\end{array}$} & \multirow[t]{2}{*}{ BUT } & \multicolumn{2}{|c|}{$\begin{array}{l}\text { Other kids never hurt themselves playing } \\
\text { sports }\end{array}$} \\
\hline $\begin{array}{l}\square \text { REALIYTRUE } \\
\text { for me }\end{array}$ & $\begin{array}{l}\text { प SORT OF TRUE } \\
\text { for me }\end{array}$ & & $\begin{array}{l}\square \text { REALY TRUE } \\
\text { for me }\end{array}$ & $\begin{array}{l}\square \text { SORT OF TRUE } \\
\text { for me }\end{array}$ \\
\hline
\end{tabular}

\begin{tabular}{|lclc|}
\hline $\begin{array}{l}\text { Some kids like to play active games outside } \\
\begin{array}{l}\square \text { REALY TRUE } \\
\text { for me }\end{array}\end{array}$ & $\begin{array}{c}\text { BUT } \\
\text { f SORT OF TRUE } \\
\text { for me }\end{array}$ & $\begin{array}{l}\text { Other kids would rather read or play video } \\
\text { games } \\
\square \text { REALY TRUE } \\
\text { for me }\end{array}$ & $\begin{array}{c}\square \text { SORT OF TRUE } \\
\text { for me }\end{array}$ \\
\hline
\end{tabular}

\begin{tabular}{|lccc|}
\hline $\begin{array}{l}\text { Some kids are among the last to be chosen } \\
\text { for active games. }\end{array}$ & BUT & Other kids are usually picked to play first. \\
$\begin{array}{l}\square \text { REALY TRUE } \\
\text { for me }\end{array}$ & $\begin{array}{c}\text { SORT OF TRUE } \\
\text { for me }\end{array}$ & $\begin{array}{c}\square \text { REALYY TRUE } \\
\text { for me }\end{array}$ & $\begin{array}{c}\square \text { SORT OF TRUE } \\
\text { for me }\end{array}$ \\
\hline
\end{tabular}

\begin{tabular}{|c|c|c|c|c|}
\hline \multicolumn{2}{|c|}{ Some kids do well in most sports } & \multirow[t]{2}{*}{ BUT } & \multicolumn{2}{|c|}{ Other kids feel they aren't good at sports } \\
\hline $\begin{array}{l}\square \text { REALLY TRUE } \\
\text { for me }\end{array}$ & $\begin{array}{l}\text { 口 SORT OF TRUE } \\
\text { for me }\end{array}$ & & $\begin{array}{l}\square \text { REALLY TRUE } \\
\text { for mee }\end{array}$ & $\begin{array}{l}\text { प SORT OF TRUE } \\
\text { for me }\end{array}$ \\
\hline \multicolumn{2}{|c|}{ Some kids learn to play active games easily } & BUT & \multicolumn{2}{|c|}{$\begin{array}{l}\text { Other kids find it hard learning to play active } \\
\text { games }\end{array}$} \\
\hline $\begin{array}{l}\text { REALLY TRUE } \\
\text { for me }\end{array}$ & $\begin{array}{l}\text { 口 SORT OF TRUE } \\
\text { for me }\end{array}$ & & $\begin{array}{l}\square \text { REALLY TRUE } \\
\text { for mee }\end{array}$ & $\begin{array}{l}\text { 口 SORT OF TRUE } \\
\text { for me }\end{array}$ \\
\hline
\end{tabular}




\begin{tabular}{|lccc|}
\hline $\begin{array}{l}\text { Some kids think they are the best at sports } \\
\begin{array}{l}\text { REALLYTRUE } \\
\text { forme }\end{array}\end{array}$ & BUT & Other kids think they aren't good at sports \\
for me & $\square$ REALLYTRUE & $\square$ SORT OF TRUE \\
for me & for me
\end{tabular}

\begin{tabular}{|c|c|c|c|c|}
\hline \multicolumn{2}{|c|}{$\begin{array}{l}\text { Some kids find games in physical education } \\
\text { hard to play }\end{array}$} & \multirow[t]{2}{*}{ BUT } & \multicolumn{2}{|c|}{$\begin{array}{l}\text { Other kids are good at games in physical } \\
\text { education }\end{array}$} \\
\hline $\begin{array}{l}\square \text { REALLY TRUE } \\
\text { for me }\end{array}$ & $\begin{array}{l}\square \text { SORT OF TRUE } \\
\text { for me }\end{array}$ & & $\begin{array}{l}\square \text { REALLY TRUE } \\
\text { for me }\end{array}$ & $\begin{array}{l}\square \text { SORT OF TRUE } \\
\text { for me }\end{array}$ \\
\hline
\end{tabular}

\begin{tabular}{|c|c|c|c|c|}
\hline \multicolumn{2}{|c|}{$\begin{array}{l}\text { Some kids like to watch games being played } \\
\text { outside }\end{array}$} & \multirow[t]{2}{*}{ BUT } & \multicolumn{2}{|c|}{$\begin{array}{l}\text { Other kids would rather play active games } \\
\text { outside }\end{array}$} \\
\hline $\begin{array}{l}\square \text { REALLY TRUE } \\
\text { for me }\end{array}$ & $\begin{array}{l}\square \text { SORT OF TRUE } \\
\text { for me }\end{array}$ & & $\begin{array}{l}\square \text { REALLY TRUE } \\
\text { for me }\end{array}$ & $\begin{array}{l}\square \text { SORT OF TRUE } \\
\text { for me }\end{array}$ \\
\hline
\end{tabular}

\begin{tabular}{|lclc|}
\hline $\begin{array}{l}\text { Some kids like to take it easy during recess } \\
\begin{array}{l}\square \text { REALLY TRUE } \\
\text { forme }\end{array}\end{array}$ & $\begin{array}{c}\text { BUT } \\
\text { forme }\end{array}$ & $\begin{array}{l}\text { Other kids would rather play active games at } \\
\text { recess }\end{array}$ \\
& $\begin{array}{l}\square \text { REALLY TRUE } \\
\text { forme }\end{array}$ & $\begin{array}{c}\square \text { SORT OF TRUE } \\
\text { for me }\end{array}$ \\
\hline
\end{tabular}

\begin{tabular}{|c|c|c|c|c|}
\hline \multicolumn{2}{|c|}{ Some kids aren't good enough for sports teams } & \multirow[t]{2}{*}{ BUT } & \multicolumn{2}{|c|}{ Other kids do well on sports teams } \\
\hline $\begin{array}{l}\square \text { REALLYTRUE } \\
\text { for me }\end{array}$ & $\begin{array}{l}\square \text { SORT OF TRUE } \\
\text { for me }\end{array}$ & & $\begin{array}{l}\square \text { REALIY TRUE } \\
\text { for me }\end{array}$ & $\begin{array}{l}\square \text { SORT OF TRUE } \\
\text { for me }\end{array}$ \\
\hline
\end{tabular}

\begin{tabular}{|lccc|}
\hline $\begin{array}{l}\text { Some kids like to read or play quiet games } \\
\begin{array}{l}\text { REALLYTRUE } \\
\text { for mee }\end{array}\end{array}$ & $\begin{array}{c}\text { BUT } \\
\text { for me }\end{array}$ & $\begin{array}{l}\text { Other kids like to play active games } \\
\square \text { REALY TRUE }\end{array}$ & $\begin{array}{c}\square \text { SORT OF TRUE } \\
\text { for me }\end{array}$ \\
\hline
\end{tabular}

\begin{tabular}{|lclc|}
\hline $\begin{array}{l}\text { Some kids like to play active games outside } \\
\text { on weekends }\end{array}$ & BUT & $\begin{array}{l}\text { Other kids like to relax and watch TV on } \\
\text { weekends }\end{array}$ \\
$\begin{array}{l}\square \text { REALYTRUE } \\
\text { forme }\end{array}$ & $\begin{array}{c}\text { a SORT OF TRUE } \\
\text { forme }\end{array}$ & $\begin{array}{c}\square \text { REALY TRUE } \\
\text { for me }\end{array}$ & $\begin{array}{c}\square \text { SORT OF TRUE } \\
\text { for me }\end{array}$ \\
\hline
\end{tabular}




\section{Appendix IX: Additional Publications}

Todd, C., Christian, D., Tyler, R., Stratton, G., \& Brophy, S. (2016). Developing HAPPEN (Health and Attainment of Pupils involved in a Primary Education Network): Working in partnership to improve child health and education. Perspectives in Public Health, 136(3), 115-116. https://doi.org/10.1177/1757913916638231 (assisted in writing and revision of the manuscript)

Marchant, E., Todd, C., Christian, D., Tyler, R., Stratton, G., \& Brophy, S. (2016). 100 Ways of Using Data to Make Lives Better. HAPPEN : Health \& Attainment of Pupils in a Primary Education Network. Farr Institute of Health Informatics Research. Available from: http://www.farrinstitute.org/publicengagement-involvement/100-ways-of-using-data-to-make-lives-better/casestudy/improving-the-health-wellbeing-and-educational-outcomes-of-primaryschool-children (assisted in writing and revision of the manuscript)

Sheldrick, M., Tyler, R., Mackintosh, K., \& Stratton, G. (2018). Relationship between Sedentary Time, Physical Activity and Multiple Lifestyle Factors in Children. Journal of Functional Morphology and Kinesiology, 3(1), 15. https://doi.org/10.3390/jfmk3010015 (collected study data, assisted in writing, analyses and revision of the manuscript) 
Appendix X: ISPAH 2016 Poster Presentation

\section{ACTIVE HEALTHY KIDS WALES REPORT CARD 2016}

IS WIAIFS TIIRNING THF TIRF ON CHII RRFN'S DHVSICAI INACTIMITV?

3. Since 2014, community and the built environment grades have dropped from B to C.

4. These results indicate that a concerted effort is required to increase phyacal activity and decrease sedentary time in children and young people in Wales.

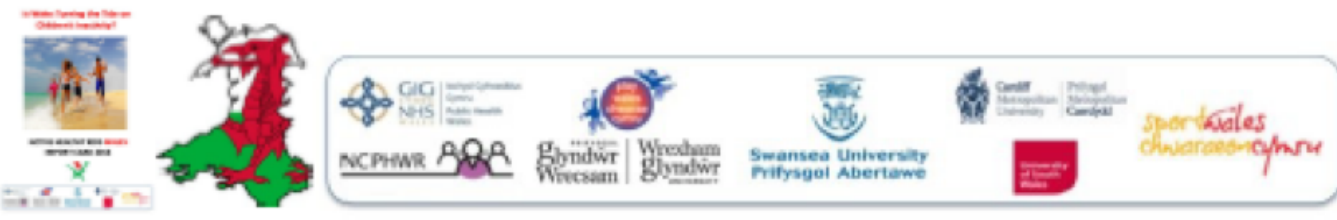




\section{Appendix XI: BASES Presentation Abstract}

TITLE: The odds of being physically competent according to fitness and weight status in 9-11 year old girls and boys

Authors: R Tyler, K Mackintosh, M McNarry, L Foweather, M Rosenberg, G Stratton.

Background: The development of physical competence (PC) across childhood plays an important role in providing the foundation skills for sustained physical activity/sporting behaviours throughout the life-course (Stodden et al. [2008]. Quest, 60, 290-306). Children with poor fitness levels have been reported to have low PC, higher body mass index (BMI) and increased risk of developing future health-related diseases. Few studies have examined these variables together.

Aim: This study aims to determine the odds ratios of BMI category (Underweight, Overweight, Obese), fitness level, and gender on physical competence category.

Methods: Following ethical approval, 95 children (56 boys; 39 girls) mean age $11.00 \pm 0.53$ years took part in the study. Stature and body mass were measured from which BMI was derived. Children completed a series of 9 motor tasks [Dragon Challenge v1.0 (DC)] in a timed circuit that included locomotion, stability and object control skills. Activities were scored on technique and completion of each task, as well as the time taken to complete the circuit. The overall score was used to calculate DC category. The children also completed the 20mMSFT (Leger and Lambert [1982]. European Journal of Applied Physiology, 49(1), 1-12), where laps completed were used to classify children at a 'Healthy' or 'Unhealthy' fitness level, using cut-off points (25 laps for girls; 33 laps for boys; Boddy et al. ([2012]. PLoS ONE, 7(9), e45755)). A cumulative odds ordinal logistic regression with proportional odds was run to determine the effect of BMI category, fitness level, and gender on the DC category achieved. 
Results: The ordinal logistic regression significantly predicted the DC category over and above the intercept-only model, $\chi^{2}(5)=17.256, P=0.004$. The odds of children who have an unhealthy level of fitness level achieving a higher DC category was $0.326(95 \% \mathrm{CI}, 0.128$ to 0.833$)$ times that of children whose scores were categorised as at a healthy fitness level, Wald $\chi_{(1)}^{2}=5.486, P=0.019$. The odds of Girls achieving a higher DC category was 0.288 (95\%CI, 0.121 to 0.688$)$ times that of Boys, Wald $\chi_{(1)}^{2}=7.862, P=0.005$. BMI category did not significantly affect DC category achieved, Wald $\chi^{2}(3)=4.048, P=0.256$.

Discussion/Conclusion: These findings show that a healthy fitness level improved the odds of achieving a higher DC category; however, BMI category did not. Moreover, the odds of girls achieving a higher category were less than a third of that of boys. Further investigation and interventions are needed on children (especially girls and those with low fitness) who have low PC, and are at risk of future diseases. 


\title{
Appendix XII: Active Healthy Kids Wales 2016 Report Card Press Release
}

\section{ACTIVE HEALTHY KIDS WALES}

\author{
PRESS RELEASE EMBARGOED TIL 12 Noon Monday July 25 2016 \\ Website release: www.activehealthykidswales.com \\ Twitter: \#AHK_Wales.
}

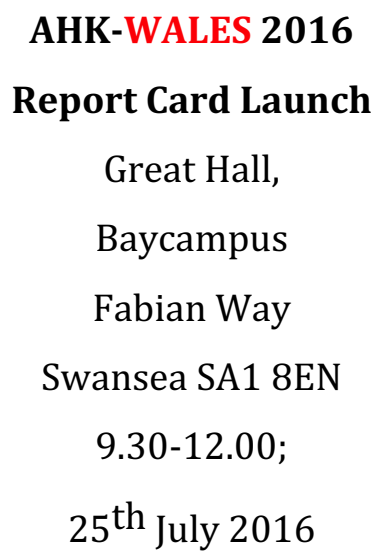

\section{Rationale}

AHK-Wales aims to monitor the lifestyles and behaviours of children. We know for example that Wales has the highest prevalence of child overweight in the UK and sedentary behavior and physical activity and fitness are amongst the worst globally. On the other hand we are pioneers in children's play, we have an active travel act and see physical literacy as a key part of a child's entitlement in the school curriculum. Further the number of children "hooked on Sport has increased. The AHK-Wales 2016 report card reports our current progress and position on children's health related physical activity. 


\section{Outline and Introduction to the AHK-Wales 2016 Report Card Launch}

This is the second Active Healthy Kids Report Card for Wales following on from the inaugural report card published in 2014. Active Healthy Kids Canada published the first report card in 2004 and celebrated the 10 $10^{\text {th }}$ anniversary in 2014 at an international global summit. Forty-two countries are currently working on their 2016 report cards that will form an "Active Healthy Kids Global Matrix " for 2016. Wales will join this matrix for the first time and will present its findings to an international audience in Bangkok in November.

Common to any report card are the grades. The AHK-Wales 2016 Report Card assigns letter grades to 10 different indicators that are grouped into two categories:

1. Strategies \& Investments; Policy, Settings \& Sources of Influence (School \& Childcare Settings, Family \& Peers, Community \& the Built Environment).

2.Behaviours that Contribute to Overall Physical Activity Levels (Active Play \& Leisure, Physical Education and Physical Activity Participation at School \& in Childcare Settings, Sedentary Behaviour, Organized Sport \& Physical Activity Participation, Active Transportation).

Letter grades (A-E) are based on an examination of current data for each indicator against a benchmark along with an assessment of changes from 2014, international comparisons and the presence of disparities (e.g., age, gender, disability, ethnicity, socioeconomic status).

Together, the indicators provide a complete and robust assessment of how Wales is doing as a country in promoting and facilitating physical activity opportunities among children and young people. 
AHK-Wales Report Card Results

\begin{tabular}{|c|c|c|c|}
\hline \multirow[b]{2}{*}{ Indicator } & \multirow{2}{*}{$\begin{array}{l}\text { Global Matrix } \\
\text { Benchmark/s } \\
\end{array}$} & \multicolumn{2}{|c|}{ AHK Grades } \\
\hline & & 2014 & 2016 \\
\hline $\begin{array}{l}\text { Sedentary } \\
\text { Behaviour }\end{array}$ & $\begin{array}{l}\% \text { of children and youth who meet sedentary } \\
\text { behavior or screen-time guidelines }\end{array}$ & D & D- \\
\hline $\begin{array}{l}\text { Physical } \\
\text { Activity }\end{array}$ & $\begin{array}{l}\% \text { of children and youth who meet physical } \\
\text { activity guidelines }\end{array}$ & D- & D- \\
\hline $\begin{array}{l}\text { Active } \\
\text { Transportation }\end{array}$ & $\begin{array}{l}\% \text { of children and youth who use active } \\
\text { transportation to get to and from places } \\
\text { (school, park, mall, friend's place) }\end{array}$ & C & C \\
\hline $\begin{array}{l}\text { Active and } \\
\text { Outdoor Play }\end{array}$ & $\begin{array}{l}\% \text { of children and youth who engage in } \\
\text { unstructured/ unorganized active play for } \\
\text { several hours a day }\end{array}$ & C & C \\
\hline $\begin{array}{l}\text { Organised } \\
\text { Sport } \\
\text { Participation }\end{array}$ & $\begin{array}{l}\% \text { of children and youth who participate in } \\
\text { organized sport and/or physical activity } \\
\text { programs }\end{array}$ & C- & C \\
\hline $\begin{array}{l}\text { Physical } \\
\text { Literacy } \\
\text { (Physical } \\
\text { Competency) }\end{array}$ & $\mathrm{N} / \mathrm{A}$ & NA & INC \\
\hline $\begin{array}{l}\text { Family and } \\
\text { Peer Influence }\end{array}$ & $\begin{array}{l}\text { - \% of parents who facilitate PA and sport } \\
\text { opportunities - \% of parents who meet the PA } \\
\text { guidelines - \% of parents who are PA with } \\
\text { their kids - \% of children with friends and } \\
\text { peers who encourage and support them to be } \\
\text { PA } \\
\text { - \% of children who encourage and support } \\
\text { their friends to be PA }\end{array}$ & D & D+ \\
\hline $\begin{array}{l}\text { Community and } \\
\text { the Built } \\
\text { Environment }\end{array}$ & $\begin{array}{l}\text { - \% of children/parents who perceive their } \\
\text { community is good at promoting PA - \% of } \\
\text { communities that report they have policies } \\
\text { promoting PA } \\
\text { - \% of communities that report infrastructure } \\
\text { specifically geared toward promoting PA - \% } \\
\text { of children/parents with facilities, programs, } \\
\text { parks, and playgrounds available } \\
\text { - \% of children/parents living in a safe } \\
\text { neighbourhood where they can be PA - \% of } \\
\text { children or parents reporting well-maintained } \\
\text { facilities, parks/playgrounds } \\
\text { - \% of children who report being outdoors for } \\
\text { several hours a day }\end{array}$ & B & C \\
\hline
\end{tabular}




\begin{tabular}{|c|c|c|c|}
\hline $\begin{array}{l}\text { National Policy, } \\
\text { Strategy and } \\
\text { Investment }\end{array}$ & $\begin{array}{l}\text { Evidence of leadership and commitment in } \\
\text { providing physical activity opportunities for } \\
\text { all children and youth Allocated funds and } \\
\text { resources for the implementation of physical } \\
\text { activity promotion strategies and initiatives } \\
\text { for all children and youth } \\
\text { Demonstrated progress through the key } \\
\text { stages of public policy making (ie, policy } \\
\text { agenda, policy formation, policy } \\
\text { implementation, policy evaluation, and } \\
\text { decisions about the future) }\end{array}$ & B- & B- \\
\hline School & $\begin{array}{l}\bullet \% \text { of schools with active school policies } \bullet \% \\
\text { of schools where the majority ( } \geq 80 \%) \text { of } \\
\text { students are taught by a PE specialist } \bullet \% \text { of } \\
\text { schools where the majority }(\geq 80 \% \text { ) of } \\
\text { students are offered at least } 150 \text { minutes of } \\
\text { PE } \bullet \% \text { of schools that offer PA opportunities } \\
\text { (excluding PE) to the majority of their } \\
\text { students } \bullet \% \text { of parents with children who } \\
\text { have access to PA opportunities at school in } \\
\text { addition to PE } \bullet \% \text { of schools with students } \\
\text { who have regular access to facilities } \\
\text { /equipment that support PA }\end{array}$ & NA & B \\
\hline
\end{tabular}




\section{ACTIVE HEALTHY KIDS WALES}

\section{Launch and schedule}

In the AHK-Wales 2016 launch we will release the report card grades and describe how we arrived at them. We aim to then engage in discussion and debate about how we use the report card as an advocacy tool with and for children and young people and those that support them in Wales. the programme for the launch is outlined below and we warmly invite you to attend and contribute and join us for lunch to celebrate the AHK-Wales 2016 report card launch.

Schedule

\begin{tabular}{|l|l|l|}
\hline Time & Indicator & Presentations \\
\hline 9.30 & $\begin{array}{l}\text { Welcome } \\
\text { Introduction Getting } \\
\text { Wales Moving AHK- } \\
\text { Wales }\end{array}$ & $\begin{array}{l}\text { Jonathon Davies } \\
\text { Welsh Government } \\
\text { Professor Gareth Stratton } \\
\text { Swansea University }\end{array}$ \\
\hline 9.45 & $\begin{array}{l}\text { Physical activity } \\
\text { levels }\end{array}$ & $\begin{array}{l}\text { Gareth Stratton/Richard Tyler } \\
\text { Swansea University }\end{array}$ \\
\hline 9.50 & $\begin{array}{l}\text { Organized sport } \\
\text { participation }\end{array}$ & $\begin{array}{l}\text { Rebecca Mattingley } \\
\text { Sport Wales }\end{array}$ \\
\hline 9.55 & $\begin{array}{l}\text { Outdoor and Active } \\
\text { Play }\end{array}$ & $\begin{array}{l}\text { Marianne Mannello } \\
\text { Play Wales }\end{array}$ \\
\hline 10.00 & $\begin{array}{l}\text { Active } \\
\text { Transportation }\end{array}$ & $\begin{array}{l}\text { Rob Sage } \\
\text { Public Health Wales }\end{array}$ \\
\hline 10.05 & Sedentary Behaviour & $\begin{array}{l}\text { Rebecca Cox } \\
\text { Welsh Government HBSC }\end{array}$ \\
\hline 10.10 & Physical Literacy & $\begin{array}{l}\text { Richard Tyler } \\
\text { Swansea University }\end{array}$ \\
\hline Doffee 10.30 & DISCUSSION + break & $\begin{array}{l}\text { Interactive discussion in pods } \\
\text { Includes coffee break Children's } \\
\text { Activity }\end{array}$ \\
\hline
\end{tabular}




\begin{tabular}{|c|c|c|}
\hline 11.00 & $\begin{array}{l}\text { Family and Peer } \\
\text { Influences }\end{array}$ & $\begin{array}{l}\text { Simon Williams } \\
\text { University of South Wales }\end{array}$ \\
\hline 11.05 & School & $\begin{array}{l}\text { Gareth Stratton } \\
\text { Swansea University }\end{array}$ \\
\hline 11.10 & $\begin{array}{l}\text { Community and built } \\
\text { environment }\end{array}$ & $\begin{array}{l}\text { Suzan Taylor } \\
\text { Glyndwr University }\end{array}$ \\
\hline 11.15 & $\begin{array}{l}\text { National Government } \\
\text { Policy and } \\
\text { Investment }\end{array}$ & $\begin{array}{l}\text { Malcolm Ward } \\
\text { Public Health Wales }\end{array}$ \\
\hline 11.25 & $\begin{array}{l}\text { Web site- Media } \\
\text { Content }\end{array}$ & $\begin{array}{l}\text { Owain Stratton Angelica Hart Lindh } \\
\text { Geoshepherds }\end{array}$ \\
\hline 11.25 & Summary & GS-Panel \\
\hline 12.00 & Lunch & $\begin{array}{l}\text { Networking Market Place Media } \\
\text { Launch }\end{array}$ \\
\hline
\end{tabular}

\section{Market Place}

The market place will take place around the great hall walkways and hall space. These will include promotion by stakeholders and research projects related to physical activity and health. The market place will be a joint event between AHKWales 2016 and ABMU Physical Activity Alliance. Market place stallholders will have access to a table and space for pull up banners and other materials. 


\title{
Appendix XIII: Ethical Approvals
}

\section{Swan-Linx application for ethical approval}

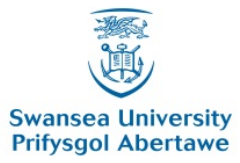

\author{
Applied Sports Technology Exercise and Medicine \\ Research Centre (A-STEM) \\ Sport and Health Portfolio, College of Engineering
}

\section{APPLICATION FOR ETHICAL COMMITTEE APPROVAL OF A RESEARCH PROJECT}

In accordance with A-STEM and College of Engineering Safety Policy, all research undertaken by staff or students linked with A-STEM must be approved by the A-STEM Ethical Advisory Committee prior to starting data collection.

Applications for approval should be submitted on this form. The researcher(s) should complete the form in consultation with the project supervisor. Where appropriate, the application must include the following appendices: (i) Participant Information Sheet, (ii) Participant Consent Form, (iii) Participant Health Screening Questionnaire.

After completing and signing the form students should ask their supervisor to complete and sign the declaration section. Staff members should submit the form directly to the Chair of the A-STEM Ethical Advisory Committee.

Applicants will be informed of the decision of the Committee via email to the project leader/supervisor.

\section{TITLE OF PROJECT}

What is the state of motor fitness and lifestyle in school children aged 9-11 years in Swansea.

\section{NAMES AND STATUS OF RESEARCH TEAM}

Sam Tuvey - Postgraduate Lead Researcher Professor Gareth Stratton - Project Director Dr Anwen Rees - Secondary Supervisor Dr Kelly Mackintosh - Secondary Supervisor Mr Cain Clark - PhD candidate Mr Nils Swindell - PhD candidate Ms Bethan Everson - MSc by Research candidate Mr Richard Tyler - MSc by Research candidate Dr Melitta McNarry - Senior lecturer Professor Sinead Brophy Project Director DeCIPHer Ms Charlotte Todd Research Assistant DeCIPHer Ms Danielle Christian Research Assistant DeCIPHer 


\section{RATIONALE}

Childhood obesity and physical inactivity is a problem within the United Kingdom with one-quarter of UK children obese by the time they leave primary school (Reilly, 2006), and fitness levels decreasing (23\% decrease from 1998-2004; Stratton et al. 2007) which is resulting in poor health and an increased risk of premature death for children in the UK. There is evidence that a high body mass index (BMI) negatively affects motor performance (Okley et al. 2010) and that taking part in physical activity can increase ability to perform motor skills (Okley et al. 2001). Although childhood obesity and physical inactivity is a national concern many parents do not recognise that their children are obese (Parry et al. 2008) which presents a problem because although the parents know how to have a healthy lifestyle they are not enforcing it (Onnerfalt et al. 2012). This research suggests that there needs to be an intervention within schools to identify overweight, inactive and obese children and to provide them with the opportunity to become physically active and learn to adopt a healthy lifestyle. The study will attempt to assess levels of motor fitness and lifestyle of school children in Swansea and provide information that could potentially allow positive changes to be made. Further, this study will seek to test the SlamTracker accelerometer (as in ethics approval code PG/2014/009). With permission, the data generated by this program may be used to develop support programs for children based on their individual needs.

\section{REFERENCES}

- Council of Europe (1983). Testing physical fitness: Eurofit: Experimental Battery: Provisional Handbook. Strasbourg: Council of Europe.

- McArdle, W.D., Katch, I. and McArdle, V.L. (2001). Exercise Physiology: Energy, Nutrition and Human Performance. Philadelphia: Lippincott Williams \& Wilkins.

- Okely, A.D., Booth, M.L. and Chey, T. (2004). Relationships between body composition and fundamental movement skills among children and adolescents. Research Quarterly for Exercise and Sport, 75, 238-247

- Okely, A.D., Booth, M.L. and Patterson, J.W. (2001). Relationship of physical activity to fundamental movement skills among adolescents. Medicine and Science in Sports and Exercise, 33, 1899-1904.

- Parry, L., Netuveli, G., Parry, J. and Saxena, S. (2008). A systematic review of parental perception of overweight status in children. Journal of Ambulatory Care Management, 31, 253-268.

- Reilly, J. (2006). Tackling the obesity epidemic: new approaches. Archives of Disease in Childhood, 9, 724-726.

- Robertson, R.J., Goss, F.L., Boer, N.F., Peoples, J.A., Foreman, A.J., Dabayebeh, I.M., et al. (2000). Children's OMNI Scale of Perceived Exertion: mixed gender and race validation. Medicine \& Science in Sports \& Exercise, 32, 452.

- Stratton, G., Canoy, D., Boddy, L.M., Taylor, S.R., Hackett, A.F., and Buchan, I.E. (2007). Cardio respiratory fitness and body mass index of 9-11 year old English children: a serial cross-sectional study from 1998-2004. International Journal of Obesity, 31, 1172-1178.

\section{AIMS and OBJECTIVES}

The aim of the project is to assess levels of motor fitness and lifestyle of children aged 9-11 years who attend 12 primary schools in Swansea. This will be done using a series of tests from the eurofit test (Council of Europe, 1983) to quantify the association with BMI. The study will also examine the gender difference and differences between the schools' BMI 
and motor fitness. Sedentary behaviours and diet will be assessed using a questionnaire. The objectives of the project are to determine whether motor fitness in school children aged 9-11 years is related to their BMI in Swansea, to determine whether there is a difference between motor fitness in boys and girls of age 9-11 years in Swansea. The study will also look at whether motor fitness is related to lifestyle behaviour in children aged 911 years in Swansea. Further, this study will seek to test the SlamTracker accelerometer (as in ethics approval code PG/2014/009).

\section{METHODOLOGY Study Design}

This study is cross-sectional and will involve male and female children aged 9-11 years who attend 12 different primary schools in Swansea. There will be approximately 700-800 children taking part, equally balanced between genders. The head teacher, parents and children are all provided information sheets and the study will be clearly explained to them, they will also be reminded that they do not have to take part in the study and can withdraw at any time without question. A written approval will be obtained from the head teacher of each school. The parents will be given a consent form that they will return if they do not wish their child to take part in the study. The children will each sign an assent form. All the subjects need to be healthy and free from disease to be able to take part in the study. The whole study will take approximately one school day in total.

The children will be asked to complete two parts for the study. The first part will be to complete an online questionnaire which will take about 30-45 minutes; this will be administered in schools by the research team. The questionnaire focuses on and asks questions regarding diet, physical activity, lifestyle, sporting interest and mental wellbeing. The last part of the study will involve a fitness fun day which will take half a day to complete. The fun day will involve a variety of motor fitness activities from the Council of Europe European Physical Fitness Test battery (Eurofit), as well as the measurement of height, sitting height and weight to determine BMI. These tests will involve a small level of exertion but this will not be more than the children would face during normal physical education lessons in school.

\section{Experimental Procedures}

The study involves completing a $\mathbf{3 0}$ minute questionnaire which will take part at the schools. The fitness fun day will take half a day to complete and will take part at Swansea University Sports Centre. The subject will be given an assent form and therefore have the option of not being involved in the study it will also be made clear to them that they can drop out at any time if they decide they do not want to take part. Active consent will be gained from the head teacher of each school, consent will be gained from the parents and assent will be gained from the children.

The online questionnaire, which has been developed by members of Swansea University, based on the Sportslinx questionnaire has 29 questions is available in Welsh and English and can be completed on computers and iPads. The questionnaire asks questions about their diet, what sports they take part in (in and out of school), what sports they would like to try, their physical activity and sedentary behaviour, their parents' physical activity. They will also provide information on their sleeping habits, what they do at school during breaktime/lunchtime, how they travel to and from school, how they feel about their fitness, health, school, friends and family. 
The fitness fun day will take place in Swansea University sports centre and will take a half a school day to complete all of the activities. The students will complete a small warm up to increase heart rate and the activities the students will be taking part in are from the eurofit test battery, which is a series of 9 motor tests covering flexibility, endurance, speed and strength. The test was devised by the Council of Europe, for children of school age and has been used in many European schools since 1988 (Council of Europe, 1983). The tests include the sit and reach test to measure flexibility, speed bounce to measure agility, standing broad jump to measure explosive leg power, the handgrip test to measure static arm strength, and two running tests, the $10 \times 5$ meter shuttle run to measure speed and agility and then $20 \mathrm{~m}$ endurance shuttle run to measure cardio respiratory endurance. During the $20 \mathrm{~m}$ shuttle run $\sim 50$ volunteers, overall, will be asked to wear the SlamTracker (Fig. 1) accelerometer (as in ethics approval code PG/2014/009). The student's height, sitting height and weight will also be measured so that $\mathrm{BMI}$ can be worked out. For the standing broad jump, speed bounce, $10 \times 5 \mathrm{~m}$ shuttle run and $20 \mathrm{~m}$ endurance shuttle run children will be asked to give their rating of exertion (RPE) using the Omni Scale of Perceived Exertion scale (Robertson et al. 2000).

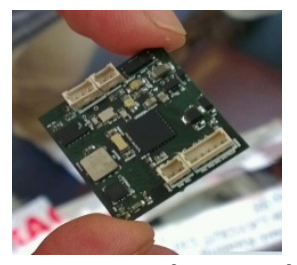

Figure. 1. SlamTracker accelerometer

\section{Data Analysis Techniques}

A Pearson's product moment correlation will be used to analyse BMI and performance in motor fitness tests. The data from the questionnaires will be entered into Excel and analysed using an analysis of variance (ANOVA). An independent T-test will be used to look for differences between motor fitness scores of male and female students. Raw acceleration data from the SlamTrackers (as in ethics approval code PG/2014/009) will be analysed using an algorithm developed from a previous study (PG/2014/009). The trace from the accelerometer, which gives acceleration in terms of ' $\mathrm{g}$ ' over the time period on the treadmill, is split into sections for clarity and ease of analysis. The data is then converted into the frequency domain by the Fast Fourier transform. The Fast Fourier transform involves taking a continuous function and representing it as a number of sine and cosine waves. It provides information about the harmonic content of a signal so that conclusions about the activity being performed can be made.

Let $x_{0}, \ldots, x_{N-1}$ be a sequence of $\mathrm{N}$ complex numbers. The Fast Fourier transform computes the Discrete Fourier transform

$X_{k}=\sum_{n=0}^{N-1} x_{n} \cdot e^{-i 2 \pi k n / N}, K \in Z$

Once the FFT has been completed on the signal and the Fourier coefficients are converted into a frequency spectrum, we are able to draw conclusions about the activity being performed based on the dominant frequency of the spectrum along with the harmonics present. Much research has been conducted characterising movements in terms of dominant frequencies (Zijlstra and Aminian, 2007), and our training data has certainly been in agreement with these findings with the most dominant frequencies during the walking section being between 1 and $3 \mathrm{~Hz}$. We then examine spectral purity of the frequency spectra and, therefore, are able to quantify how energy is distributed across the 
spectrum. We have begun to investigate links between spectral purity and walking styles.

We are also able to use the spectrum to determine how much background noise is present in our signals. Results from the analysis of the spectrum are then used to inform the second part of our algorithm.

The second part of our algorithm involves using the frequency domain as a tool to correct for noise and 'idealise' the data to some extent, making use of a filter centred on the dominant frequency and prominent harmonics. Such a task would be much more difficult in the time domain. We chose those harmonics that are $\geq 10 \%$ of the height of the dominant peak, however, this is something that we will seek to optimise and tailor to an individual. The idealised data is then converted back into the time domain via the inverse Fast Fourier transform, resulting in an idealised time signal. Using double integration, we can convert our acceleration data into displacement information and effectively trace out the path of a subject walking on a treadmill or performing other tasks whilst wearing an accelerometer on their body.

\section{Storage and Disposal of Data and Samples}

The completed questionnaires and fitness fun day data will be kept private and confidential, hard copies of information will be kept in a secure office and any personal information will be changed into a number rather than a name. All files on a computer will be password protected and files with personal details will only be accessible by only accessible by Sam Tuvey, Professor Gareth Stratton and Dr Anwen Rees and Mr Cain Clark, Mr Nils Swindell, Ms Bethan Everson and Mr Richard Tyler. The schools will have access to the anonymous data for educational and curriculum use. The data is part of a longitudinal study and will not be disposed of. Data may be also used, with consent, in further data linkage projects.

\section{LOCATION OF THE PREMISES WHERE THE RESEARCH WILL BE CONDUCTED.}

The fitness fun day will take place on the premises of Swansea University - the running track facility, and the administering of the questionnaire will take place at the respective schools' premises. The research team, Sport Wales Sport Ambassadors and a member(s) of City and County of Swansea sport and recreation team will all be present. The session will be overseen by school teachers

\section{PARTICIPANT RISKS AND DISCOMFORTS}

The students will not be put under any greater risk than what they would face when in a PE class in school. However, there is a possibility that whilst taking part in the fitness fun day they will get an injury, feel sick or faint. A first aid trained researcher (Mr Cain Clark, Mr Nils Swindell) and/or member of the sports centre running track staff will always be present during any activity. There will also be an automatic defibrillator stored on site (on the wall upon entrance) in case any cases of sudden death syndrome. All sports centre staff are defibrillator trained and will be responsible for the defibrillators safe operation. All anthropometric measures will be taken sensitively. All lead researchers will have a DBS check and no researcher will be on their own with any individual child. 


\section{INFORMATION SHEET AND INFORMED CONSENT}

The submission should be specific about the type of consent that will be sought, which should be indicated below:

- Have you included a Participant Information Sheet for the participants of the study? YES/NO

- Have you included a Participant Consent Form for the participants of the study? YES/NO

\section{COMPUTERS}

- Are computers to be used to store data? YES/NO

- If so, is the data registered under the Data Protection Act? YES/NO

\section{STUDENT DECLARATION}

Please read the following declarations carefully and provide details below of any ways in which your project deviates from these. Having done this, each student listed in section 2 is required to sign where indicated.

- "I have ensured that there will be no active deception of participants.

- I have ensured that no data will be personally identifiable.

- I have ensured that no participant should suffer any undue physical or psychological discomfort

- I certify that there will be no administration of potentially harmful drugs, medicines or foodstuffs.

- I will obtain written permission from an appropriate authority before recruiting members of any outside institution as participants.

- I certify that the participants will not experience any potentially unpleasant stimulation or deprivation.

- I certify that any ethical considerations raised by this proposal have been discussed in detail with my supervisor.

- I certify that the above statements are true with the following exception(s):" Student/Researcher signature: (include a signature for each student in research team) Date:

\section{SUPERVISOR'S DECLARATION}

In the supervisor's opinion, this project (delete those that do not apply):

- "Does not raise any significant issues.

- Raises some ethical issues, but I consider that appropriate steps and precautions have been taken and I have approved the proposal.

- Raises ethical issues that need to be considered by the Departmental Ethics Committee.

- Raises ethical issues such that it should not be allowed to proceed in its current form."

Supervisor's signature:

Date: 
The ethical issues raised by this project have been considered by the A-STEM Ethical Advisory Committee.

Please refer to the summary comments noted in the decision email sent to the project lead/supervisor. Please ensure that you take account of these comments.

Where necessary, please prepare a revised submission that should be shown to your supervisor (for student applications) and then resubmitted to the A-STEM Ethical Advisory Committee for reconsideration.

Application number

Date:

Signed:

(Chair, A-STEM Ethical Advisory Committee) 
written above.

\section{What will happen to the children if they take part?}

If you decide to allow your pupils to take part in the study they will come to a fitness fun day at the university with the rest of their class. This will involve a half day of fun physical activities which will measure the children's strength, speed, agility, endurance and flexibility. These activities include; $20 \mathrm{~m}$ Multi Stage shuttle runs, $10 \times 5 \mathrm{~m}$ shuttle runs, grip

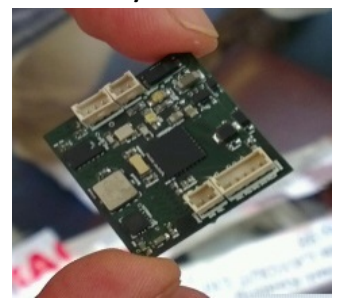
strength, sit and reach, standing broad jump, and speed bounce (for more information about these activities, please contact anyone from the research team listed). During these activities, some of the children may be asked to wear the SlamTracker accelerometer (Fig. 1). This device will measure how fast the child moves forwards and backwards, side to side and up and down, and will only be worn during the fitness fun day activities. The children will also be asked

Fig. 1: SlamTracker Accelerometer to rate on a scale how much effort they exerted in the speed bounce, standing broad jump, $10 \times 5 \mathrm{~m}$ shuttle run and $20 \mathrm{~m}$ Multi Stage shuttle run. The activities will be no harder than what a child would do during school PE lessons. The children will also have other measurements taken including; weight, height and sitting height. All measures of body size are taken privately and no results are shared with the rest of the class. Children can choose not to have their anthropometric measurements taken if they do not want to be measured. We have followed this approach with around 70000 children in Liverpool since 1996.

On a separate day the children will also be asked complete a questionnaire at your school, which will take about 30 minutes. There will be members of the research team and teachers present to assist the children in filling them in.

\section{What are the possible disadvantages of taking part?}

Taking part in the fitness fun day poses no greater risk than a child would face during PE lessons in school. However, in the unlikely event that a child feels unwell, sick, tired, faint or sustains an injury, there will be people monitoring the children during all parts of the fitness fun day, and the children's teachers will remain present at all times. A qualified first aider will always be present at the sports centre during the fitness fun day.

\section{What are the possible benefits of taking part?}

The fun day will be an active and very enjoyable day for the children and they will get to take part in a variety of different activities that they might not have taken part in before. They will be able to find out about their skills, fitness, and flexibility related to health and well-being. The final data will be made available to the school for educational and curriculum use.

\section{Will my taking part in the study be kept confidential?}

All the data we collect from the children will be kept private and confidential; the children's names will be changed to numbers. Any hard copies of the questionnaires and fun day data will be kept in a secure office and computer files with any personal information will be password protected. The data obtained will only be looked at by responsible individuals of the research team from Swansea University and the City \& County of Swansea or from regulatory authorities where it is relevant to your child's participation in the research. The data we collect will be used to assess the health, motor fitness and lifestyle of children in Swansea.

\section{What if I have any questions?}

If you have any questions about what I have written above or anything to do with the 
study please don't hesitate to contact me or anyone from the research team as detailed above. If after the study you are concerned about how any aspect of the research was conducted please contact the Chair of the College Ethics Committee, Professor Mike McNamee 


\section{Applied Sports Technology Exercise and Medicine Research Centre (A-STEM) \\ Sport and Health Portfolio, College of Engineering}

\section{HEADTEACHER CONSENT FORM}

(Version 2.1, Date: $\_/ 20$

\section{Project Title:}

Health, motor skills, fitness and lifestyle of Swansea School Children aged 9-11 years old

\section{Contact Details:}

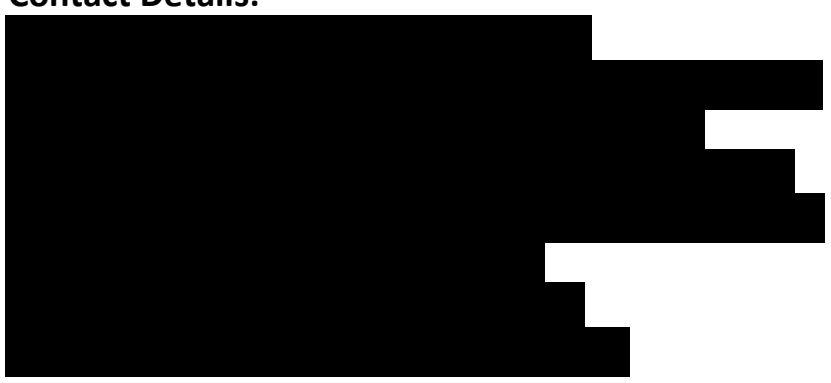

\section{Please initial box}

1. I confirm that I have read and understood the information sheet dated ___ 20_ (Version number 2.1) for the above

study and have had the opportunity to ask questions.

2. I understand that the children's participation is voluntary and that I am free to withdraw my pupils at any time, without giving any reason, without their medical care or legal rights being affected.

3. I understand that sections of the data obtained may be looked at by responsible individuals from the Swansea University or from regulatory authorities where it is relevant to the children's taking part in research. I give permission for these individuals to have access to these records.

4. I am happy for any data collected in this study to be used in future health related studies where data collected will be linked to health outcomes.

I agree to allow the pupils in my school to take part in the above study.
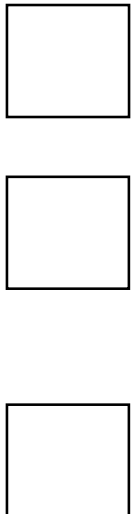

Name of School

Date

Signature

Name of Headteacher

Date

Signature

Researcher

Date

Signature 


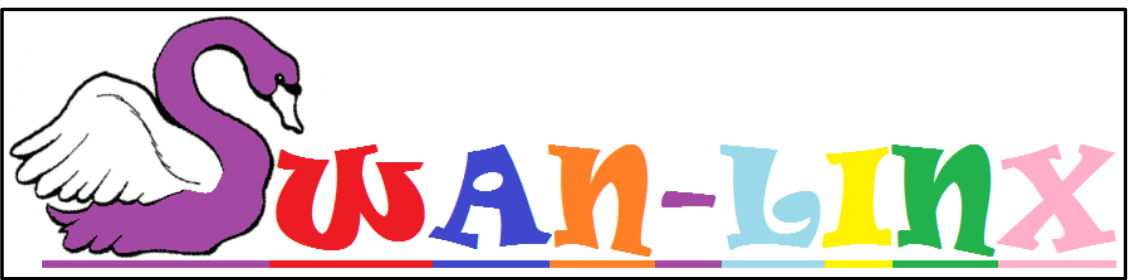

PARENT INFORMATION SHEET

(Version 2.1, Date: $/ 20$

Project Title:

Health, motor skills, fitness and lifestyle of Swansea School Children aged 9-11

years old

\section{Contact Details:}

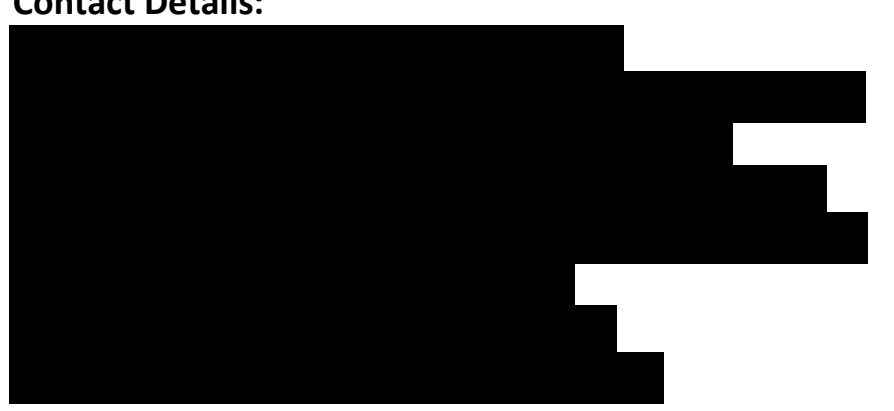

Please read the information below carefully before deciding whether to consent for your child's participation.

\section{Invitation Paragraph}

The children in your child's school who are aged 9-11 years have been invited to take part in a study led by Swansea University and City and County of Swansea called Swan-Linx. Many other schools in the Swansea area are also taking part. Your child will be asked to take part in a fitness fun day and to complete a questionnaire. The data we collect from your child will help us assess different physical and psychological aspects of children in Swansea.

\section{What is the purpose of the study?}

The purpose of the study is to investigate the health, motor skills (physical competency), fitness and lifestyle of children aged 9-11 years in Swansea schools and also to test the SlamTracker accelerometer. The data collected will be used in a postgraduate student's thesis and will assist the City \& County of Swansea in tracking children's health and physical activity, to decide how best to help children become healthier and more involved in sport and physical activity in the future.

\section{Why have I been chosen?}

Your child has been invited to take part in both the fitness fun day and questionnaire, because they attend a primary school in Swansea and are aged 9 to 11 years. During the 
study if your child does not feel happy about anything that they are asked to do they can stop at anytime, without fear of penalty. If you need any more information about the study then please contact any member of the team on the details above.

\section{What will happen to your child if they take part?}

Your child will attend a fitness fun day at the University with the rest of their class. This will involve a half day of fun physical activities which will measure children's strength, speed, agility, endurance and flexibility. These activities include; $20 \mathrm{~m}$ Multi Stage shuttle runs, 10x5m shuttle run, handgrip strength, sit and reach, standing broad jump, and speed bounce (for more information about any of these activities, please contact anyone from the research team listed).

During the activities, some of the children may be asked to wear the SlamTracker accelerometer (Fig. 1).

This device will measure how fast your child moves forwards and backwards, side to side and up and down, and will only be worn

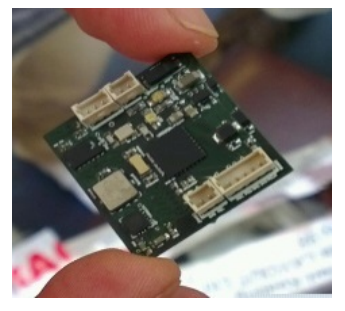
during the fitness fun day activities. The children will also be asked to rate on a scale $(1=$ extremely easy $\rightarrow 10=$ extremely hard) how difficult they found the speed

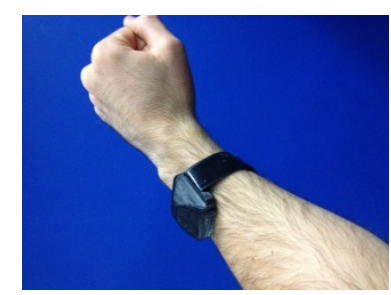
bounce, standing broad jump, $10 \times 5 \mathrm{~m}$ shuttle run and $20 \mathrm{~m}$ Multi Stage shuttle run.

Your child will also have some other measurements taken including; Fig. 1: SlamTracker Accelerometer weight, height and sitting height. All measures of body size are taken privately and no results are shared with the rest of the class, however, your child will not have to have them taken if they do not want to. All activities will be no harder than your child would do during school PE lessons. On a separate day your child will also be asked complete a questionnaire at their school about their health, physical activity and lifestyle, which will take about 30 minutes. There will be members of the research team and teachers present to assist your child in filling them in.

\section{What are the possible disadvantages of taking part?}

Taking part in the fitness fun day poses no greater risk than a child would face during PE lessons in school. However, in the unlikely event that a child feels unwell, sick, tired, faint, or sustains an injury there will be people monitoring the children during all parts of the fitness fun day, and the children's teachers will remain present at all times. A qualified first aider will always be present at the sports centre during the fitness fun day.

\section{What are the possible benefits of taking part?}

The fun day will be an active and very enjoyable day for the children and they will get to take part in a variety of different activities that they might not have taken part in before. They will be able to find out about their skills, fitness, and flexibility related to health and well-being. The final data will be made available to the school for educational and curriculum use.

\section{Will my taking part in the study be kept confidential?}

All the data we collect from your child will be kept private and confidential; the children's names will be changed to numbers. Any hard copies of the questionnaires and fun day data will be kept in a secure office and computer files with any personal information will be password protected. The data obtained will only be looked at by responsible individuals of the research team from Swansea University and the City \& County of Swansea or from regulatory authorities where it is relevant to your child's participation in the research. The 
data we collect will be used to assess the health, motor skills, fitness and lifestyle of children in Swansea.

\section{What if I have any questions?}

If you have any questions about what is written above or anything to do with the study please don't hesitate to contact me or anyone from the research team (see contact details above). If after the study you are concerned about how any aspect of the research was conducted please contact the Chair of the College Ethics Committee, Professor Mike McNamee 


\section{Applied Sports Technology Exercise and Medicine Research Centre (A-STEM) \\ Sport and Health Portfolio, College of Engineering}

PARENT CONSENT FORM

(Version 2.1, Date: $\_/ / 20$

\section{Project Title:}

Health, motor skills, fitness and lifestyle of Swansea School Children aged 9-11

years old

\section{PLEASE RETURN THIS FORM TO SCHOOL TO CONSENT FOR YOUR CHILD TO TAKE PART IN THE PROJECT.}

\section{Contact Details:}

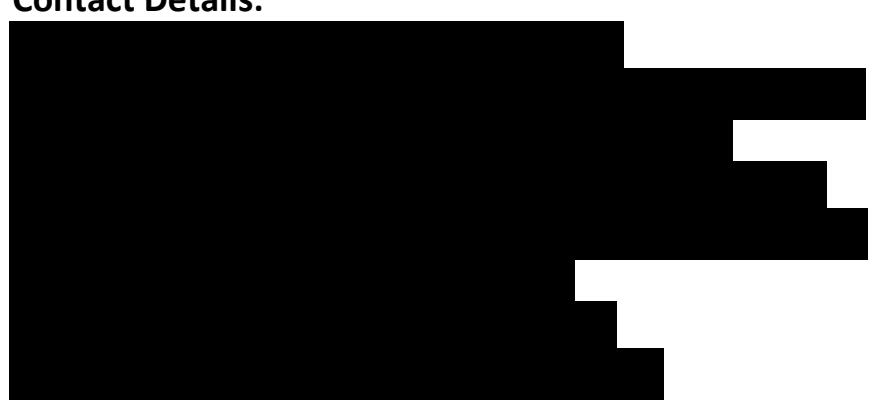

\section{Please INITIAL each box below}

1. I confirm that I have read and understood the information sheet dated _/20_ (Version number 2.1) for the above study and have had the opportunity to ask questions.

2. I am happy for my child to participate in the Fitness Fun Day, and wear the SlamTracker accelerometer

3. I understand that my child's participation is voluntary and that I am free To withdraw my child at any time, without giving any reason, without their medical care or legal rights being affected.

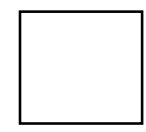

I understand that sections of the data obtained may be looked at by responsible individuals from the Swansea University or from regulatory authorities where it is relevant to the children's taking part in research. I give permission for these individuals to have

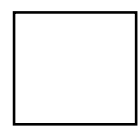
access to these records.

5. I am happy for any data collected in this study to be used in future health related studies where data collected will be linked to health outcomes. I agree to allow my child to take part in the above study.

Name of Child (participant)

Name of Parent/Guardian

Researcher

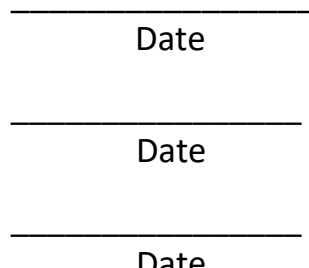

Date
Signature

Signature
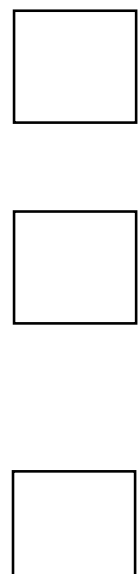


\section{Applied Sports Technology Exercise and Medicine Research Centre (A-STEM) \\ Sport and Health Portfolio, College of Engineering}

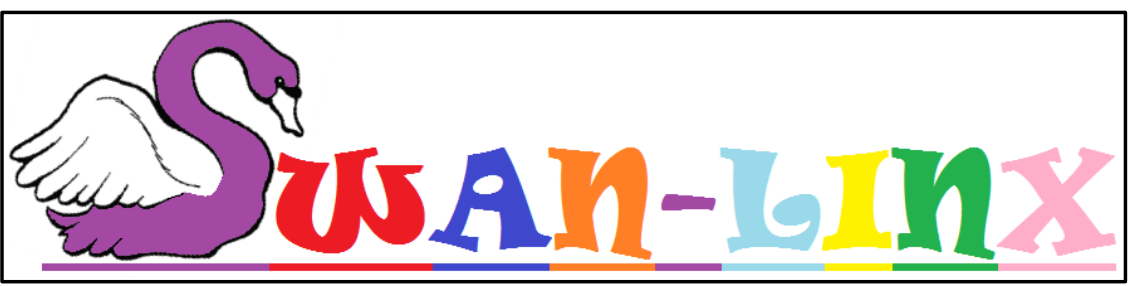

PARTICIPANT INFORMATION SHEET

(Version 2.1, Date:___/20_)

\section{Project Title:}

Health, motor skills, fitness and lifestyle of Swansea School Children aged 9-11

years old

\section{Contact Details:}

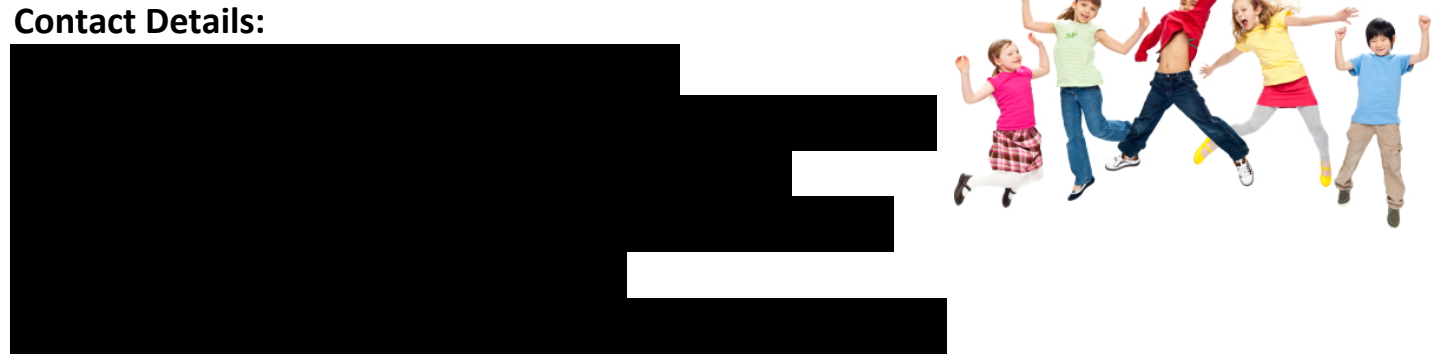

You have been selected to take part in a Swansea University study. You have been chosen because you are between the ages of 9-11 years old and go to a primary school in Swansea.

In this study you have the chance to take part in a fitness fun day at the University and on another day the research team will come into your school and ask you to complete a questionnaire about your health and lifestyle and about how physically active you are. Physical activity is any movement that requires your body to work harder than it does whilst sitting, or resting.

The fitness fun day will involve lots of fun physical activities that you may not have tried before which should be really enjoyable. These include running, jumping and strength tests.

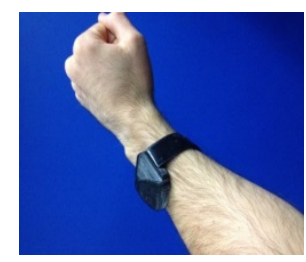

You may also get asked to wear our BRAND NEW devices, and use the NEWEST technology to measure how fast you move forwards and backwards, side to side and up and down.

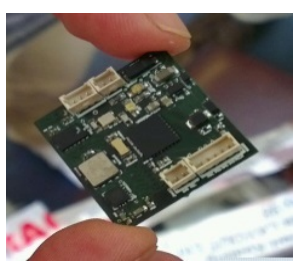
You will not be forced to do any of the activities and can stop at any time without fear of penalty or having to worry about being

in trouble.

If you have any questions please ask any of the research team.

THANK YOU. 

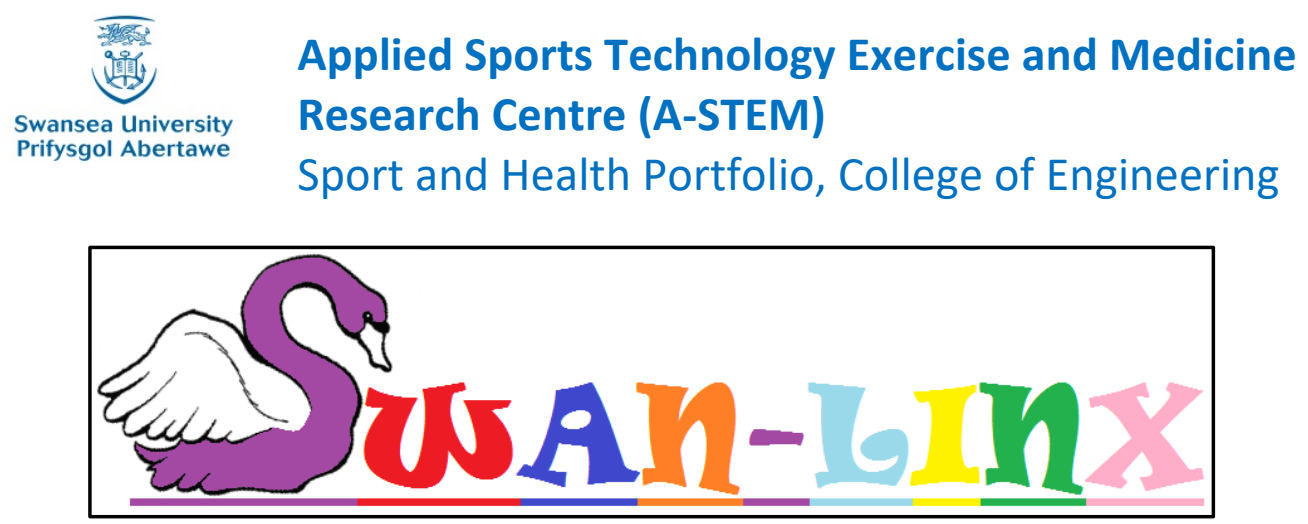

PARTICIPANT ASSENT FORM

(Version 2.1, Date: _/_/20_)

Project Title:

Health, motor skills, fitness and lifestyle of Swansea School Children aged 9-11 years old

Contact Details:

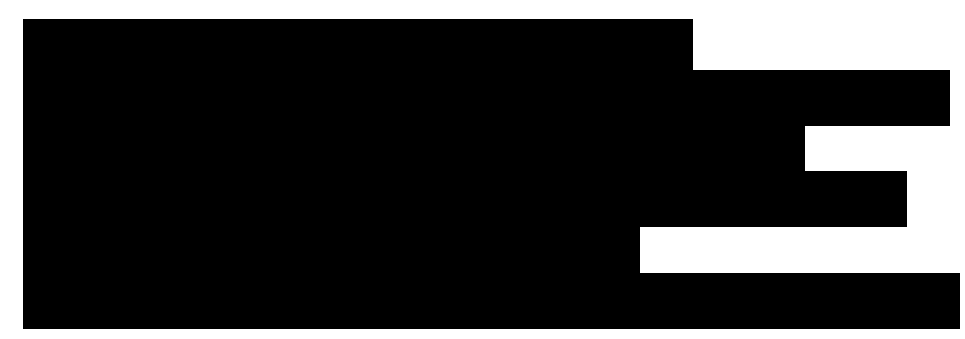

I agree to take part in this study. 


\title{
Swan-Linx and Dragon Challenge application for ethical approval
}

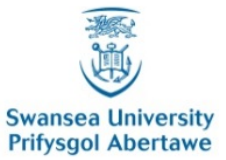

\author{
Applied Sports Technology Exercise and Medicine \\ Research Centre (A-STEM) \\ Sport and Health Portfolio, College of Engineering
}

\section{APPLICATION FOR ETHICAL COMMITTEE APPROVAL OF A RESEARCH PROJECT}

In accordance with A-STEM and College of Engineering Safety Policy, all research undertaken by staff or students linked with A-STEM must be approved by the A-STEM Ethical Advisory Committee prior to starting data collection.

Applications for approval should be submitted on this form. The researcher(s) should complete the form in consultation with the project supervisor. Where appropriate, the application must include the following appendices: (i) Participant Information Sheet, (ii) Participant Consent Form, (iii) Participant Health Screening Questionnaire.

After completing and signing the form students should ask their supervisor to complete and sign the declaration section. Staff members should submit the form directly to the Chair of the A-STEM Ethical Advisory Committee.

Applicants will be informed of the decision of the Committee via email to the project leader/supervisor.

\section{TITLE OF PROJECT}

What is the state of motor skills (physical competency), fitness and lifestyle in children aged $8-13$ years in Wales?

\section{NAMES AND STATUS OF RESEARCH TEAM}

Professor Gareth Stratton - Project Director

Dr Kelly Mackintosh - Secondary Supervisor

Mr Richard Tyler - PhD candidate

Mr Cain Clark - PhD candidate

Ms Claire Barnes - PhD candidate

Mr Nils Swindell - PhD candidate

Dr Melitta McNarry - Senior lecturer

Professor Sinead Brophy - Project Director DeCIPHer

Ms Charlotte Todd - Research Assistant DeCIPHer

Ms Danielle Christian - Research Assistant DeCIPHer

Mr Luke Martin - MSc by research candidate

Mr Michael Sheldrick - MSc by research candidate

Ms Tabatha Pace - BSc dissertation student

Mr Andrew Beveridge - BSc dissertation student 


\section{RATIONALE}

Childhood obesity and physical inactivity is a problem within the United Kingdom with one-quarter of UK children being classed as obese by the time they leave primary school (Reilly, 2006). Fitness levels are decreasing (23\% decrease from 1998-2004; Stratton et al. 2007) which is resulting in poor health and an increased risk of premature death for children in the UK. There is evidence that a high body mass index (BMI) negatively affects motor performance (Okley et al. 2010) and that taking part in physical activity can increase ability to perform motor skills (Okley et al. 2001). Although childhood obesity and physical inactivity is a national concern many parents do not recognise that their children are obese (Parry et al. 2008) which presents a problem because although the parents know how to have a healthy lifestyle they are not promoting it (Onnerfalt et al. 2012).In 2008, Stodden et al. created a conceptual model that identifies fundamental movement skills as being a key underlying factor that can influence participation in physical activity and, consequently, health status. These are the foundation skills that, if mastered, form the platform for motor skill competence (physical competency). The authors explain that higher physical competency levels will cause a positive trajectory toward perceived competence, health-related fitness and, sequentially, physical activity levels. This in turn, will promote the child to maintain a healthy weight. More recently, authors have reviewed the growing literature around the conceptual model (Robinson et al. 2015). This review confirmed that evidence shows motor skill competence (physical competency) is positively associated with perceived competence and multiple aspects of health such as, physical activity, cardio-respiratory fitness, muscular strength and endurance, and a healthy weight status. Therefore, understanding the relationships between factors that influence children's health and well-being could aid in addressing the current declining levels of fitness and high levels of obesity.

This research project seeks to quantify motor fitness, physical competency, physical activity, and health related behaviours, and seek to establish interrelationships between them. Further, this study will use an accelerometer (REC PG/2014/009) to generate raw, unfiltered data to analyse the movements of children in this context. The data generated by this programme will be used to develop support programmes for children based on their individual needs.

\section{REFERENCES}

- Council of Europe (1983). Testing physical fitness: Eurofit: Experimental Battery: Provisional Handbook. Strasbourg: Council of Europe.

- McArdle, W.D., Katch, I. and McArdle, V.L. (2001). Exercise Physiology: Energy, Nutrition and Human Performance. Philadelphia: Lippincott Williams \& Wilkins.

- Okely, A.D., Booth, M.L. and Chey, T. (2004). Relationships between body composition and fundamental movement skills among children and adolescents. Research Quarterly for Exercise and Sport, 75, 238-247

- Okely, A.D., Booth, M.L. and Patterson, J.W. (2001). Relationship of physical activity to fundamental movement skills among adolescents. Medicine and Science in Sports and Exercise, 33, 1899-1904.

- Parry, L., Netuveli, G., Parry, J. and Saxena, S. (2008). A systematic review of parental perception of overweight status in children. Journal of Ambulatory Care Management, 31, 253-268.

- Reilly, J. (2006). Tackling the obesity epidemic: new approaches. Archives of Disease in Childhood, 9, 724-726.

- Robertson, R.J., Goss, F.L., Boer, N.F., Peoples, J.A., Foreman, A.J., Dabayebeh, 
I.M., et al. (2000). Children's OMNI Scale of Perceived Exertion: mixed gender and race validation. Medicine \& Science in Sports \& Exercise, 32, 452.

- Robinson, L. E., Stodden, D. F., Barnett, L. M., Lopes, V. P., Logan, S. W., Rodrigues, L. P., \& D'Hondt, E. (2015). Motor competence and its effect on positive developmental trajectories of health. Sports Medicine, 45(9), 1273-1284.

- Stodden, D. F., Goodway, J. D., Langendorfer, S. J., Roberton, M. A., Rudisill, M. E., Garcia, C., \& Garcia, L. E. (2008). A developmental perspective on the role of motor skill competence in physical activity: An emergent relationship. Quest, 60(2), 290306.

- Stratton, G., Canoy, D., Boddy, L.M., Taylor, S.R., Hackett, A.F., and Buchan, I.E. (2007). Cardio respiratory fitness and body mass index of 9-11 year old English children: a serial cross-sectional study from 1998-2004. International Journal of Obesity, 31, 1172-1178.

\section{AIMS and OBJECTIVES}

The aim of the project is to establish levels of motor fitness, physical competency and lifestyle behaviours of children who attend schools in Wales. This will be done using a series of tasks from the Eurofit test battery (Council of Europe, 1983) and the Dragon Challenge V1.0 activities. Sedentary behaviours, diet and wellbeing will be assessed using an online questionnaire (the CHAT tool). The study will also examine correlates of motor skills, physical competency, physical activity, lifestyle and fitness. There are many objectives, for example: To determine the relationship between motor fitness and BMI. To quantify the differences between motor skills and fitness in boys and girls. To establish whether motor skills and fitness, are related to lifestyle behaviours. To determine the general level of motor fitness, physical competency, health, wellbeing and lifestyle of children in Wales. Further, this study will seek to use the SlamTracker accelerometer to determine specific signals related to physical activity, fitness and motor performance (as in ethics approval code PG/2014/009).

\section{METHODOLOGY}

\section{Study Design}

This study will involve boys and girls aged 8-13 years schools in Wales. The children will be asked to complete three parts for the study.

1. The first part will be to complete an online questionnaire (CHAT) which will take about 30-45 minutes; this will be administered on schools premises' by the research team. The questionnaire focuses on and asks questions regarding diet, physical activity, lifestyle, sporting interest and mental wellbeing.

2. The second part of the study will involve a fitness fun day that will take half a day to complete. The fun day will involve a variety of motor fitness activities from the Council of Europe European Physical Fitness Test battery (Eurofit), as well as the measurement of height, sitting height and weight to determine BMI.

3. The children will complete further motor performance assessments in "dragon challenge" (third part). The dragon challenge includes 9 motor skill assessments completed serially. These further quantify motor fitness/fundamental movement tasks, including; running, jogging, balancing on a bench, balancing on the spot, basketball dribbling, jumping, catching and throwing. Dragon challenge is completed in 3 minutes. These tasks involve exertion commensurate with physical education lesson demands. 


\section{Experimental Procedures}

(1) Completion of the CHAT tool (online questionnaire); (2) Fitness Fun Day tasks; (3) Dragon Challenge Tasks. The study involves completing a 30-45 minute questionnaire which will take part at the school. The fitness fun day will take half a day to complete and will take part at respective schools premises (sports halls) e.g. Pentrehafod school sports hall, and Swansea University indoor training centre. The participant will be given an assent form and therefore the choice of opting out of all or part of the project. Active consent will be gained from the head teacher of each school, consent will be gained from the parents and assent will be gained from the children. The online questionnaire, which has been developed by members of Swansea University, based on the Sportslinx questionnaire has 29 questions is available in both Welsh and English and can be completed on computers, iPads and tablets. The questionnaire includes items on diet, sport participation, physical activity and sedentary behaviour, parents' physical activity and lifestyle behaviours including sleep times, mode of travel to and from school, and perceptions about their fitness, health, school, friends and family. The fitness fun day will take place in the sports hall of the respective schools or Swansea University's indoor training centre and will take a half a school day to complete all of the activities. The students will complete a small warm up to increase heart rate and the activities the students will be taking part in are from the Eurofit test battery and Dragon Challenge activities. The Eurofit test was devised by the Council of Europe, for children of school age and has been used in many European schools since 1988 (Council of Europe, 1983). The Dragon Challenge activities were developed by Swansea and Edgehill Universities and Sport Wales. In sum the tasks include the sit and reach test to measure flexibility, speed bounce to measure agility, standing broad jump to measure explosive leg power, the handgrip test to measure static arm strength, and running tests, the $10 \times 5$ meter shuttle run to measure speed and agility and then $20 \mathrm{~m}$ endurance shuttle run to measure cardio respiratory endurance, all of these activities are commensurate with physical education lessons within a school. Additionally participants will take part in "Dragon Challenge" activities, which include; running, jumping over hurdles, balancing, core agility/flexibility, ball throwing, ball catching and ball bouncing. Throughout, participants will be asked to wear the SlamTracker accelerometer (as in ethics approval code PG/2014/009 \& PG14/2014/007). The student's height, sitting height and weight will also be measured, by a member of the listed research team, so that BMI and maturation offset can be calculated. For some activities the participants may be asked to give their rating of exertion (RPE) using the Omni Scale of Perceived Exertion Scale (Robertson et al. 2000). During the activities, after attaining consent from parent/guardian, school, and participant, a video recording device will be used to assess movements during the motor fitness activities, post-hoc. Four cameras will be placed along the anteroposterior and mediolateral planes to capture the movements in detail. The cameras will be placed to capture the activities, but will not impede the participants' movements during the motor fitness activities. These video recordings will be used to further analyse the motor fitness/fundamental movement skills of the participants.

\section{Data Analysis Techniques}

A Pearson's product moment correlation will be used to analyse BMI and performance in motor fitness tests. The data from the questionnaires will be entered into Excel and analysed using an analysis of variance (ANOVA). An independent T-test will be used to look for differences between motor fitness scores of male and female students. Postal code data will be used to portray a 'map' of areas/districts, enabling analysis of how socioeconomic status and WIMD scores relate to levels of health and motor fitness in children. Raw acceleration data from the SlamTrackers (as in ethics approval code 
PG/2014/009) will be analysed using an algorithm developed from a previous study (PG/2014/009 \& PG14/2014/007). The trace from the accelerometer, which gives acceleration in terms of ' $\mathrm{g}$ ' over the time period, is split into sections for clarity and ease of analysis. The data is then converted into the frequency domain by the Fast Fourier transform. The Fast Fourier transform involves taking a continuous function and representing it as a number of sine and cosine waves. It provides information about the harmonic content of a signal so that conclusions about the activity being performed can be made. Data will be analysed using MatLab, with time and frequency features from the raw acceleration trace extracted. Video recordings will be used to analyse and compare the motor fitness activities and also to compare/confirm assessor ratings.

\section{Storage and Disposal of Data and Samples}

The completed questionnaires and fitness fun day (including Dragon Challenge) data will be kept private and confidential, hard copies of information will be kept in a secure office and any personal information will be changed into a number rather than a name. All files on a computer will be password protected and files with personal details will only be accessible by only accessible by Professor Gareth Stratton, Dr Kelly Mackintosh, Dr Melitta McNarry, Mr Cain Clark, Ms Claire Barnes, Mr Nils Swindell, Mr Richard Tyler, Mr Luke Martin, Mr Michael Sheldrick, Ms Tabatha Pace and Mr Andrew Beveridge. The schools will have access to the anonymous data for educational and curriculum use. The data is part of a longitudinal study and will not be disposed of. Data may be also used, with consent, in further data linkage projects. This involves linking the collected data to health and education outcomes using Secure Anonymised Information Linkage (SAIL) through the Information Governance Review Panel (IGRP). Any and all video recorded data will be kept separate from any identifying documents and will be managed under the Data Protection Act 1998, concluding each day of testing, video recordings will be transferred to a password encrypted device. The video recorded data will be kept for educational purposes, such as for training, and demonstrating the motor fitness activities to future participants. Alternatively, any video recorded data not used for academic or educational purposes will be destroyed by the lead investigator, Richard Tyler, overseen by project director Gareth Stratton, after no longer than 7 years.

\section{LOCATION OF THE PREMISES WHERE THE RESEARCH WILL BE CONDUCTED.}

The fitness fun day will take place on the premises of Swansea University's indoor training centre, or at the respective schools' premises/sports hall (for example, Pentrehafod School sports hall). The research team, Sport Wales Sport Ambassadors, volunteers, and a member(s) of City and County of Swansea sport and recreation team will all be present. The session will also be overseen by school teachers, and a qualified first aider (either Swansea indoor training centre staff, or school sports hall attendant)

\section{PARTICIPANT RISKS AND DISCOMFORTS}

The students will not be put under any greater risk than what they would face when in a PE class in school. However, there is a possibility that whilst taking part in the fitness fun day they will get an injury, feel sick or faint. A first aid trained staff will always be present during any activity. All anthropometric measures will be taken sensitively. All lead researchers will have a DBS check and no researcher will be on their own with any individual child. 


\section{INFORMATION SHEET AND INFORMED CONSENT}

The submission should be specific about the type of consent that will be sought, which should be indicated below:

- $\quad$ Have you included a Participant Information Sheet for the participants of the study? YES/NO

- $\quad$ Have you included a Participant Consent Form for the participants of the study? YES/NO

\section{COMPUTERS}

- $\quad$ Are computers to be used to store data?

YES/NO

- If so, is the data registered under the Data Protection Act?

YES/NO

\section{STUDENT DECLARATION}

Please read the following declarations carefully and provide details below of any ways in which your project deviates from these. Having done this, each student listed in section 2 is required to sign where indicated.

- "I have ensured that there will be no active deception of participants.

- I have ensured that no data will be personally identifiable.

- I have ensured that no participant should suffer any undue physical or psychological discomfort

- I certify that there will be no administration of potentially harmful drugs, medicines or foodstuffs.

- I will obtain written permission from an appropriate authority before recruiting members of any outside institution as participants.

- I certify that the participants will not experience any potentially unpleasant stimulation or deprivation.

- I certify that any ethical considerations raised by this proposal have been discussed in detail with my supervisor.

- I certify that the above statements are true with the following exception(s):"

Student/Researcher signature: (include a signature for each student in research team) Date:

\section{SUPERVISOR'S DECLARATION}

In the supervisor's opinion, this project (delete those that do not apply):

- "Does not raise any significant issues.

- $\quad$ Raises some ethical issues, but I consider that appropriate steps and precautions have been taken and I have approved the proposal.

- $\quad$ Raises ethical issues that need to be considered by the Departmental Ethics Committee.

- $\quad$ Raises ethical issues such that it should not be allowed to proceed in its current form."

Supervisor's signature:

Date: 


\section{ETHICS COMMITTEE DECISION (COMMITTEE USE ONLY) ETHICAL APPROVAL: REC PG/2014/37 \\ GRANTED/REJECTED}

The ethical issues raised by this project have been considered by the A-STEM Ethical Advisory Committee.

Please refer to the summary comments noted in the decision email sent to the project lead/supervisor. Please ensure that you take account of these comments.

Where necessary, please prepare a revised submission that should be shown to your supervisor (for student applications) and then resubmitted to the A-STEM Ethical Advisory Committee for reconsideration.

Application number

Date:

Signed:

(Chair, A-STEM Ethical Advisory Committee) 


\section{Applied Sports Technology Exercise and Medicine Research Centre (A-STEM) \\ Sport and Health Portfolio, College of Engineering}

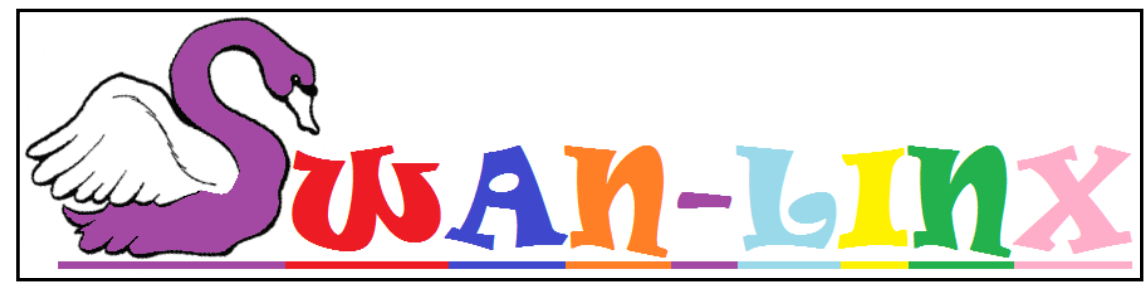

PARENT INFORMATION SHEET

(Version 1.0, Date: /20_1

Project Title:

Health, motor skills (physical competency), fitness and lifestyle of School Children in Wales

\section{Contact Details:}

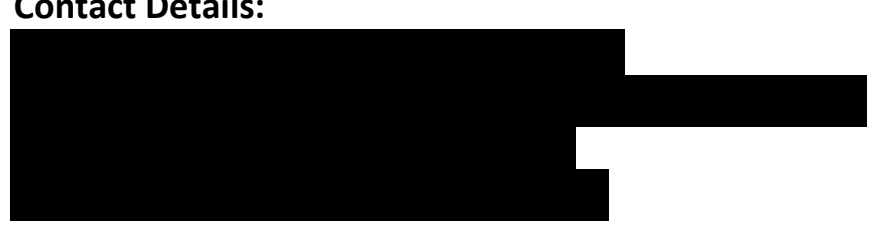

\section{Please read the information below carefully before deciding whether to consent for your child's participation.}

\section{Invitation Paragraph}

The children in your child's class have been invited to take part in a new study that will look at the relationships between lifestyle behaviours, such as, sitting time, computer game play, fitness, sleep time, type of foods eaten and so forth. The data we collect from all of the children taking part in the study will help us assess different aspects of children's motor skills (physical competency), fitness and lifestyle.

\section{What is the purpose of the study?}

The purpose of this study is to investigate motor skills (physical competency), fitness and lifestyle in children. The study will also test an accelerometer which measures body movement. The data collected will be used in a postgraduate student's thesis and will assist in tracking children's health, physical activity and physical competency, to decide how best to help children become healthier and more involved in sport and physical activity in the future. The data collected will also be used to map results across Wales. This will help us to further analyse levels of health and fitness in children in terms of demographics.

\section{Why has my child been chosen?}

All of the children in your child's class, including your child, have been invited to take part in the Fitness Fun Day. During the day, if your child does not feel happy about anything that they are asked to do, they can stop at any time, without fear of penalty. If you need 
any more information about the study then please contact any member of the team on the details above.

\section{What will happen to your child if they take part?}

Your child will attend a Fitness Fun Day with the rest of their class. This will involve a half day of fun physical activities which will measure children's strength, speed, agility, endurance and flexibility. These activities include; $20 \mathrm{~m}$ shuttle run (measures endurance), $10 \times 5 \mathrm{~m}$ sprint (measures speed and agility), handgrip (measures strength), sit and reach (measures flexibility), standing long jump (measures leg explosive power), and speed bounce (measures leg speed, agility and endurance). They will also take part in the Dragon Challenge V1.0; this Challenge assesses many fundamental movement skills. During the Dragon Challenge, your child and their class will perform 9 discrete tasks in a circuit that require single or combinations of motor skills in order to achieve a goal. These tasks involve running, jumping over hurdles, balancing, core agility/flexibility, ball throwing, ball catching and ball bouncing.

During the physical activities, some of the children may be asked to wear the SlamTracker accelerometer. This device will measure how fast your child moves forwards and backwards, side to side and up and down.

Your child will also have weight, height and sitting height measurements recorded, taken by a member of the research team listed. Measures of body weight are taken privately in a separate room or behind a screen, there will always be other children in the room or next to the screen but they will not be party to the results or be able to view the measures and no results are shared with the rest of the class, however, your child will not have to have them taken if they do not want to. All activities will be no harder than your child would do during school PE lessons. Finally, during the Fitness Fun Day session or at your child's school, your child will also be asked complete a questionnaire, about their health, physical activity and lifestyle, which will take about 30 minutes. There will be members of the research team and teachers present to assist your child in filling them in. All the activities during the Fitness Fun Day are aimed for the children's enjoyment and not as a competition. With your permission, a video camera will also be used to capture some of the activities the children are performing.

\section{What are the possible disadvantages of taking part?}

Taking part in the Fitness Fun Day and Dragon Challenge activities poses no greater risk than a child participating in school physical education lessons. However, in the unlikely event that a child feels unwell, there will be people monitoring the children during all parts of the fitness fun day and dragon challenge activities, and the children's teachers will remain present at all times. A qualified first aider will always be present during the Fitness Fun Day (including Dragon Challenge).

\section{What are the possible benefits of taking part?}

The Fitness Fun Day will be an active and very enjoyable day for the children and they will get to take part in a variety of different activities that they might not have taken part in before. They will be able to find out about their skills and fitness in relation to health and well-being. Further we want children to engage with their results. Therefore school data will be anonymised and made available to the school for educational purposes.

\section{Will my child taking part in the study be kept confidential?}

All the data we collect from your child will be kept private and confidential; the children's 
names will be changed to numbers. Any hard copies of the questionnaires and fitness fun day data will be kept in a secure office and computer files with any personal information will be password protected. The data obtained will only be looked at by responsible individuals of the research team from Swansea University, City \& County of Swansea, and the PLPS team (Sport Wales), or from regulatory authorities where it is relevant to your child's participation in the research. To enable us to track changes in heath over time we will also keep the secure data available for future linkage with other sets of data collected in the future such as GP visits or educational results for example.

\section{What if I have any questions?}

If you have any questions about what is written above or anything to do with the study please don't hesitate to contact me or anyone from the research team (see contact details above). If after the study you are concerned about how any aspect of the research was conducted please contact the Chair of the College Ethics Committee, Professor Mike McNamee 


\section{Applied Sports Technology Exercise and Medicine Research Centre (A-STEM) \\ Sport and Health Portfolio, College of Engineering}

\section{PARENT CONSENT FORM}

(Version 1.0, Date: ____/20__

\section{Project Title:}

Health, motor skills (physical competency), fitness and lifestyle of School Children in Wales

\section{PLEASE RETURN THIS FORM TO SCHOOL TO CONSENT FOR YOUR CHILD TO TAKE PART IN THE PROJECT.}

\section{Contact Details:}

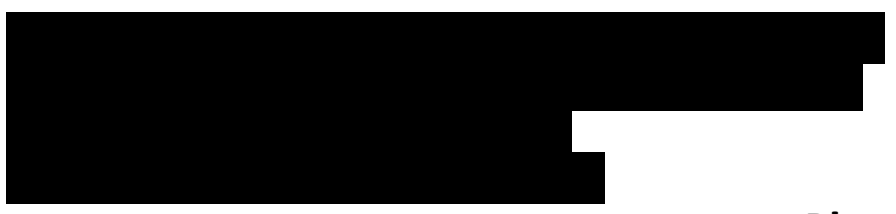

\section{Please INITIAL each box below}

1. I confirm that I have read and understood the information sheet dated ___ 20 _ (Version number 1.0) for the above study and have had the opportunity to ask questions.

2. I am happy for my child to participate in the Fitness Fun Day, and wear the SlamTracker accelerometer.

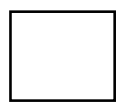

3. I understand that my child's participation is voluntary and that I am free to withdraw my child at any time, without giving any reason, without their medical care or legal rights being affected.

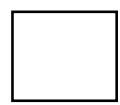

4. I understand that sections of the data obtained may be looked at by responsible individuals from the Swansea University or from regulatory authorities where it is relevant to the children's taking part in research

5. I am happy for any data collected in this study to be used in future health related studies where data collected will be linked to health outcomes and educational records.

I am happy for the activities to be video recorded for academic use ONLY.

I agree to allow my child to take part in the above study.
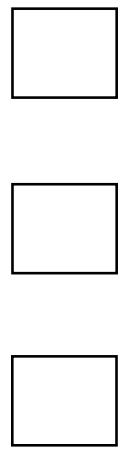

Name of Participant (child)

Name of Parent/Guardian

Researcher

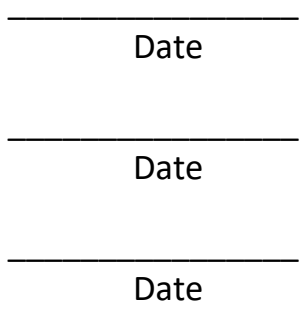

Date
Signature

Signature 

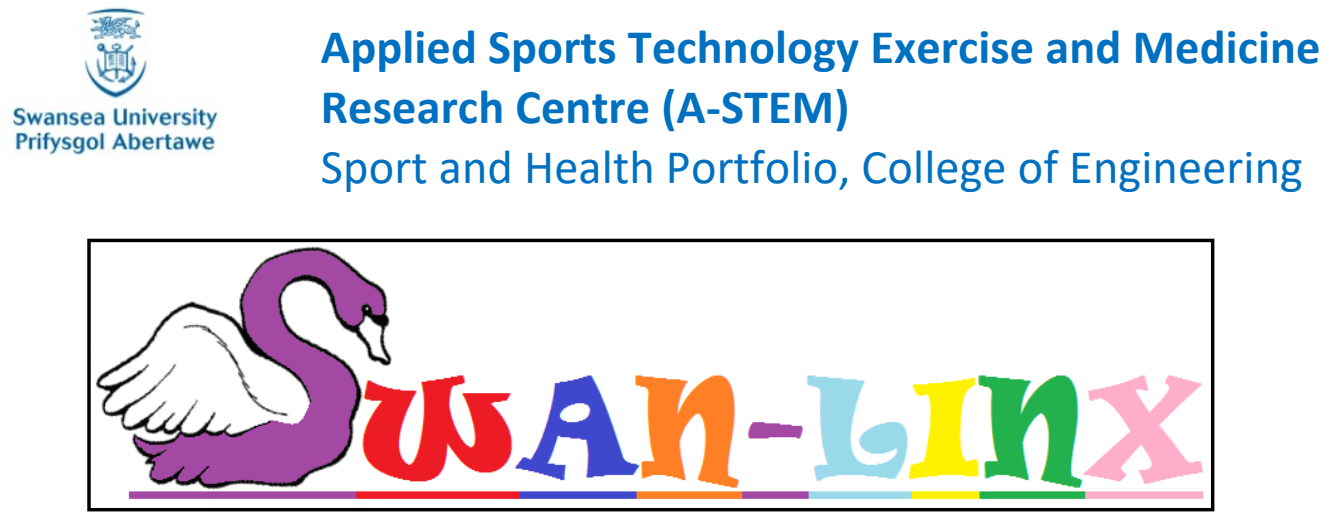

HEADTEACHER INFORMATION SHEET

(Version 1.0, Date:___/20__)

\section{Project Title:}

Health, motor skills (physical competency), fitness and lifestyle of Swansea School Children

\section{Contact Details:}

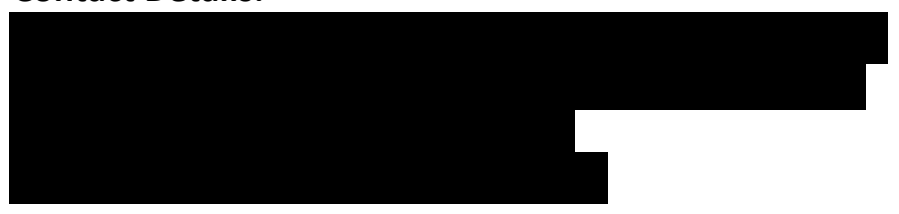

\section{Invitation Paragraph}

The children in your school have been invited to take part in a new study that will look at the relationships between lifestyle behaviours, such as, sitting time, computer game play, fitness, sleep time, type of foods eaten and so forth. Other schools are also taking part in the study. They will be asked to take part in a Fitness Fun Day, where they will complete the Dragon Challenge, and a series of physical activities and a questionnaire. The data we collect will help us assess different aspects of children's motor skills (physical competency), fitness and lifestyle. Please consider whether you are able to commit to the requirements stated below before signing the consent form.

\section{What is the purpose of the study?}

The purpose of this study is to investigate the health, motor skills (physical competence), fitness, lifestyle of children from selected schools. The study will also test an accelerometer which measures body movement. The data collected will be used in a postgraduate student's thesis and will assist in tracking children's health, physical activity and physical competency, to decide how best to help children become healthier and more involved in sport and physical activity in the future. The data collected will also be used to map results across Wales. This will help us to further analyse levels of health and fitness in children in terms of demographics.

\section{Why have I been chosen?}

The children in your school have been invited take part in the Fitness Fun Day as they 
attend school in Wales. During the day if any of the children do not feel happy about anything they are asked to do, they can stop at any time, without fear of penalty. If you need any more information about the study then please contact any member of the team on the details above.

\section{What will happen to the children if they take part?}

Your pupils will attend a Fitness Fun Day; this will involve a half day of fun physical activities which will measure the children's strength, speed, agility, endurance and flexibility. These activities include; $20 \mathrm{~m}$ Multi Stage shuttle runs (measures endurance), $10 \times 5 \mathrm{~m}$ sprint (measures speed and agility), handgrip (measures strength), sit and reach (measures flexibility), standing long jump (measures leg explosive power), and speed bounce (measures leg speed, agility and endurance). They will also take part in the Dragon Challenge V1.0; this Challenge assesses many fundamental movement skills. During the Dragon Challenge, the children will perform 9 discrete tasks in a circuit that require single or combinations of motor skills in order to achieve a goal. These tasks involve running, jumping over hurdles, balancing, core agility/flexibility, ball throwing, ball catching and ball bouncing.

During these physical activities, some of the children may be asked to wear a sensor called a SlamTracker accelerometer. This device will measure how fast the child moves forwards and backwards, side to side and up and down, and will only be worn during some activities. The activities will be no harder than what a child would do during school PE lessons. The children will also have weight, height and sitting height measurements recorded, take by a member of the research team listed. Measures of body weight are taken privately in a separate room or behind a screen, there will always be other children in the room or next to the screen but they will not be party to the results or be able to view the measures and no results are shared with the rest of the class. Children can choose not to have their anthropometric measurements taken if they do not want to be measured. We have followed this approach with around 70000 children in Liverpool since 1996. Finally, during the Fitness Fun Day session, the children will also be asked complete a questionnaire, about their health, physical activity and lifestyle, which will take about 30 minutes. There will be members of the research team and teachers present to assist the children in filling them in. All the activities during the Fitness Fun Day are aimed for the children's enjoyment and not as a competition. With your permission, a video camera will also be used to capture some of the activities the children are performing.

\section{What are the possible disadvantages of taking part?}

Taking part in the Fitness Fun Day and Dragon Challenge activities poses no greater risk than a child would face during physical education lessons in school. However, in the unlikely event that a child feels unwell, there will be people monitoring the children during all parts of the fitness fun day and dragon challenge activities, and the children's teachers will remain present at all times. A qualified first aider will always be present during the fitness fun day (including Dragon Challenge).

\section{What are the possible benefits of taking part?}

The Fitness Fun Day will be an active and very enjoyable day for the children and they will get to take part in a variety of different activities that they might not have taken part in before. They will be able to find out about their skills and fitness in relation to health and well-being. Further we want children to engage with their results. Therefore school data 
will be anonymised and made available to the school for educational purposes.

\section{Will my taking part in the study be kept confidential?}

All the data we collect from the children will be kept private and confidential; the children's names will be changed to numbers. Any hard copies of the questionnaires and fun day data will be kept in a secure office and computer files with any personal information will be password protected. The data obtained will only be looked at by responsible individuals of the research team from Swansea University and the City \& County of Swansea, and the PLPS team (Sport Wales), or from regulatory authorities where it is relevant to the children's participation in the research. To enable us to track changes in heath over time we will also keep the secure data available for future linkage with other sets of data collected in the future such as GP visits or educational results for example.

\section{What if I have any questions?}

If you have any questions about what I have written above or anything to do with the study please don't hesitate to contact me or anyone from the research team as detailed above. If after the study you are concerned about how any aspect of the research was conducted please contact the Chair of the College Ethics Committee, Professor Mike McNamee 


\section{Applied Sports Technology Exercise and Medicine Research Centre (A-STEM) \\ Sport and Health Portfolio, College of Engineering}

\section{HEADTEACHER CONSENT FORM}

(Version 1.0, Date:

/20

\section{Project Title:}

Health, motor skills (physical competency), fitness and lifestyle of School Children in Wales

\section{Contact Details:}

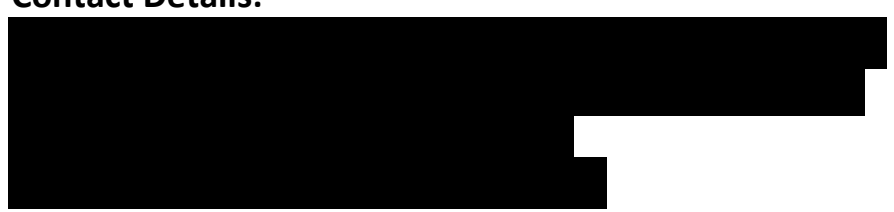

\section{Please INITIAL each box below}

1. I confirm that I have read and understood the information sheet dated ___ 20_ (Version number 1.0) for the above study and have had the opportunity to ask questions.

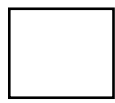

2. I understand that the children's participation is voluntary and that I am free to withdraw my pupils at any time, without giving any reason, without their medical care or legal rights being affected.

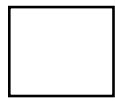

3. I understand that sections of the data obtained may be looked at by responsible individuals from the Swansea University or from regulatory authorities where it is relevant to the children's taking part in research.

4. I am happy for any data collected in this study to be used in future health related studies where data collected will be linked to health outcomes and educational records.

5. I am happy for the activities to be video recorded for academic use ONLY.
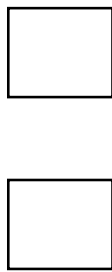

I agree to allow the pupils in my school to take part in the above study.

Name of School

Name of Headteacher

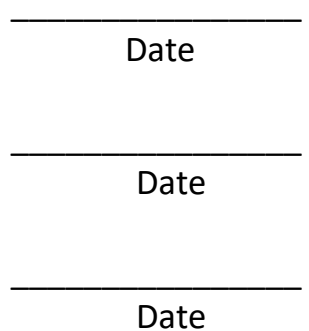

Date
Signature

Signature

\begin{tabular}{c}
\hline Signature \\
\hline Signature \\
\hline Signature
\end{tabular}



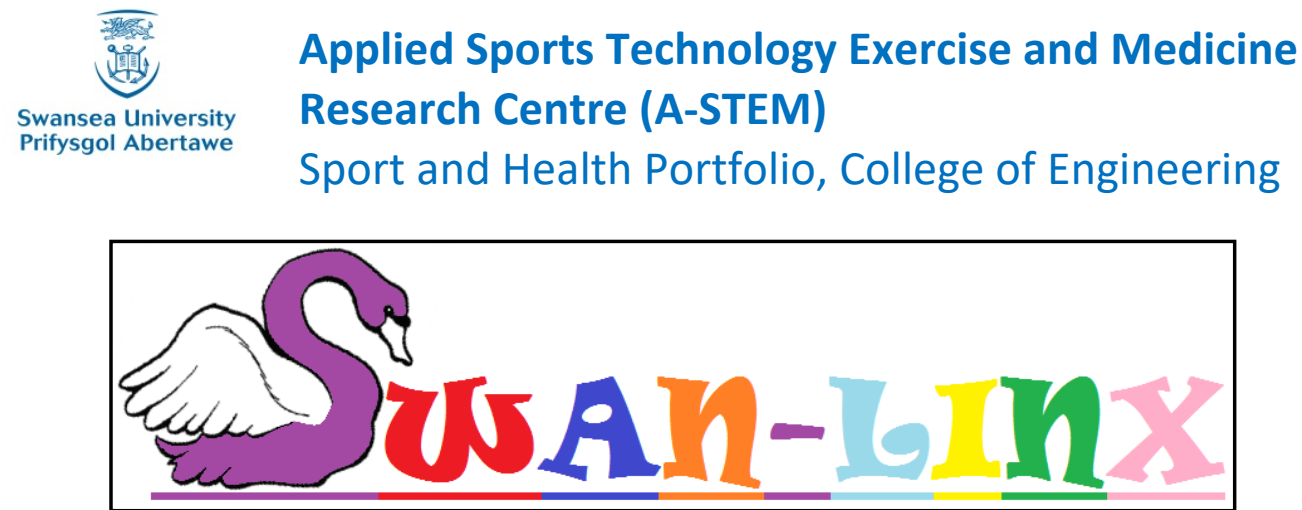

PARTICIPANT INFORMATION SHEET

(Version 1.0, Date:

(20_-

Project Title:

Health, motor skills (physical competency), fitness and lifestyle of School Children in Wales

Contact Details:
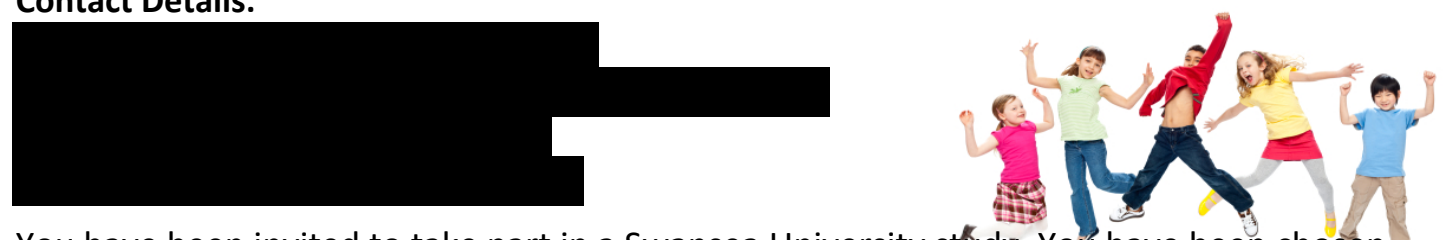

You have been invited to take part in a Swansea University study. You have been chosen because you are between the ages of 8-13 years old and go to a school in Wales.

In this study you have the chance to take part in a fitness fun day, the Dragon Challenge, and complete a questionnaire about your health and lifestyle and about how physically active you are. Physical activity is any movement that requires your body to work harder than it does whilst sitting, or resting. The fitness fun day will involve lots of fun physical activities that you may not have tried before which should be really enjoyable. These activities will include a $20 \mathrm{~m}$ shuttle run to see how long you can run for, a shuttle sprint to see how quick you can run back and forth, gripping with your hands to find out your strength, sit and reach to find out how flexible you are, standing long jump to see how far you can jump, and speed bounce to see how many times you can jump in 30 seconds. The Dragon Challenge involves running, jumping over hurdles, balancing, core agility/flexibility, ball throwing, ball catching and ball bouncing. These are done in a circuit and should be really fun.

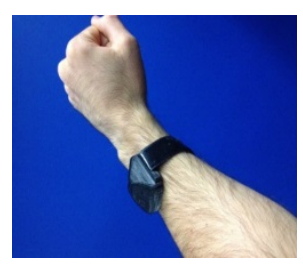

You may also get asked to wear our BRAND NEW devices, and use the NEWEST technology to measure how fast you move forwards and backwards, side to side and up and down. This will be worn on your wrist and ankle during some of the physical activities. You may also be video recorded during some of the physical activities. This will help us see how well you have done.

You will not be forced to do any of the activities and can stop at any time without fear of penalty or having to worry about being in trouble.

If you have any questions please ask.

\section{THANK YOU.}




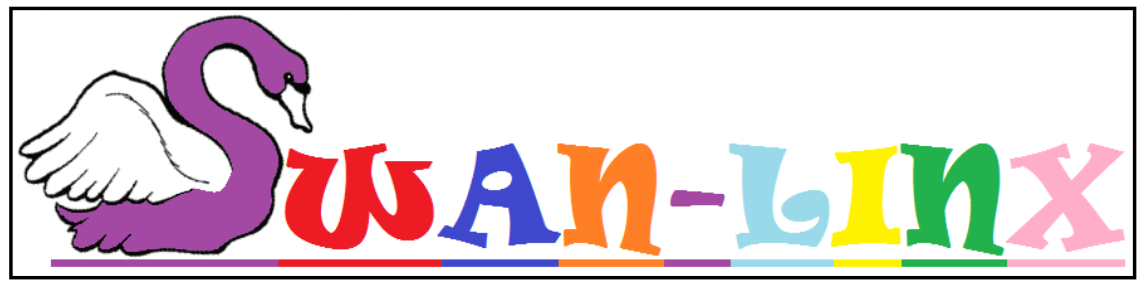

\section{PARTICIPANT ASSENT FORM}

\section{(Version 1.0, Date:___/20_)}

Project Title:

Health, motor skills (physical competency), fitness and lifestyle of School Children in Wales

\section{Contact Details:}

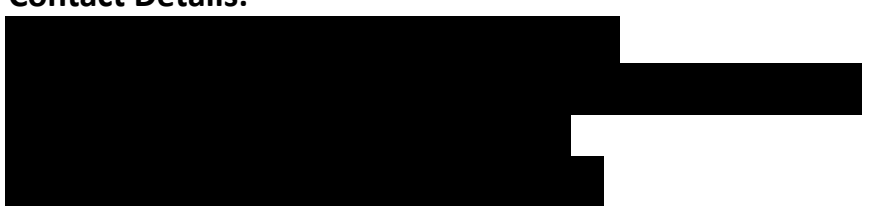

\begin{tabular}{|c|c|}
\hline & $\begin{array}{l}\text { Please look at the following statements and put your initials } \\
\text { if you agree. Example Sarah Jones: SJ }\end{array}$ \\
\hline 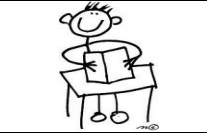 & I have read the Participant Information Sheet \\
\hline & I understand what I will be doing if I take part \\
\hline & I have had a chance to think about taking part \\
\hline & I have had a chance to ask any questions \\
\hline & $\begin{array}{c}\text { I agree that my data can be used in a research report and } \\
\text { that I will not be named so } \\
\text { no-one will know it was my information }\end{array}$ \\
\hline & $\begin{array}{l}\text { I understand that all of the information } \\
\text { will be kept private and only shared with the research team }\end{array}$ \\
\hline c) & $\begin{array}{c}\text { I am happy to try to do the activities that the Swan-Linx } \\
\text { Team wants me to do during the fitness fun day, and } \\
\text { complete the questionnaire. }\end{array}$ \\
\hline$\because$ & I am happy to take part in this study \\
\hline
\end{tabular}

Name of Participant

Date

Signature

Researcher

Date

Signature 


\section{Validating the Dragon Challenge application for ethical approval}

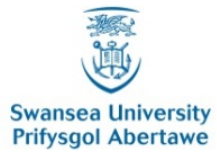

Applied Sports Technology Exercise and Medicine Research Centre (A-STEM)

Sport and Health Portfolio, College of Engineering

\section{APPLICATION FOR ETHICAL COMMITTEE APPROVAL OF A RESEARCH PROJECT}

In accordance with A-STEM and College of Engineering Safety Policy, all research undertaken by staff or students linked with A-STEM must be approved by the A-STEM Ethical Advisory Committee prior to starting data collection.

Applications for approval should be submitted on this form. The researcher(s) should complete the form in consultation with the project supervisor. Where appropriate, the application must include the following appendices: (i) Participant Information Sheet, (ii) Participant Consent Form, (iii) Participant Health Screening Questionnaire.

After completing and signing the form students should ask their supervisor to complete and sign the declaration section. Staff members should submit the form directly to the Chair of the A-STEM Ethical Advisory Committee.

Applicants will be informed of the decision of the Committee via email to the project leader/supervisor.

\section{TITLE OF PROJECT}

The validity and reliability of the Dragon Challenge V1.0 in measuring physical competency in children aged 8-14 years.

\section{NAMES AND STATUS OF RESEARCH TEAM}

Professor Gareth Stratton - Project Director

Dr Kelly Mackintosh - Secondary Supervisor

Mr Richard Tyler - PhD candidate

Mr Cain Clark - PhD candidate

Ms Claire Barnes - PhD candidate

Mr Nils Swindell - PhD candidate

Dr Melitta McNarry - Senior lecturer

Mr Luke Martin - MSc by research candidate

Mr Michael Sheldrick - MSc by research candidate

Ms Tabatha Pace - BSc dissertation student

Mr Andrew Beveridge - BSc dissertation student

Professor Sinead Brophy

Ms Charlotte Todd

Ms Danielle Christian 


\section{RATIONALE}

A physically active lifestyle is extremely important to a child's health (Janssen and LeBlanc, 2010). Physical literacy is the motivation, confidence, physical competence, knowledge and understanding to value and take responsibility for engagement in physical activities for life (Whitehead, 2010). The ability to monitor children's progression throughout their physical literacy journey across the life-course requires a broad spectrum of valid and reliable methods. Notably, the physical competence domain of physical literacy includes not only fitness, but also physical movement competence.

Stodden et al. (2008) created a conceptual model that identifies fundamental movement skills as being a key underlying factor that can influence participation in physical activity and, consequently, health status. These are the foundation skills that, if mastered, form the platform for motor skill competence. The authors explain that higher physical competency levels will cause a positive trajectory toward perceived competence, healthrelated fitness and, sequentially, physical activity levels.

Among older children and adolescents, the strength of physical movement competency and physical activity relationship becomes increasingly more important. Children, who are not proficient in locomotor, object control and stability skills, are less likely to adopt an active lifestyle (Lubans et al, 2013; Lloyd et al, 2014, Robinson et al. 2015). Thus, movement competence is an increasingly important determinant of physical literacy in children.

Current assessments of movement skill require children to perform each skill in isolation (Henderson et al 2010; Folio \& Fewell 2000, Ulrich 2000), making them time and resource intensive to administer (Wiart \& Darah, 2001). Over the past 2 years Swansea University have worked with Sport Wales to design an assessment of physical competence in a continuous circuit of 9 tasks.

\begin{tabular}{|l|l|l|}
\hline \multicolumn{2}{|l|}{ Task } & Description \\
\hline 1. & Balance bench & $\begin{array}{l}\text { Walk the length of the narrow side of a bench beam, completing a } 360 \\
\text { degree turn at mark before dismounting at the end of the bench. }\end{array}$ \\
\hline 2. & Core agility & $\begin{array}{l}\text { Complete } 4 \text { body shape positions (dish - arch - dish - arch), rotating the } \\
\text { body in both directions. }\end{array}$ \\
\hline 3. & Wobble spot & $\begin{array}{l}\text { Complete } 5 \text { bean bag 'passes' around the body whilst balancing on the } \\
\text { wobble spot on one leg. }\end{array}$ \\
\hline 4. & Overarm throw & $\begin{array}{l}\text { Throw a tennis ball, using an overarm throw, at a target approximately } 9 \\
\text { metres away. }\end{array}$ \\
\hline 5. & Basketball dribble & $\begin{array}{l}\text { Using either hand, dribble a basketball around } 4 \text { coloured spots } \\
\text { positioned in a 'z' formation. }\end{array}$ \\
\hline 6. & Catch & Catch a tennis ball thrown underarm at a rebound net from any distance. \\
\hline 7. & Jumping patterns & $\begin{array}{l}\text { Complete a jumping pattern sequence that includes a series of hops and } \\
\text { jumps (2 footed jump over hurdle } \rightarrow 2 \text { footed landing } \rightarrow 2 \text { left hops } \rightarrow 2 \\
\text { right hops } \rightarrow 2 \text { foot jump over hurdle } \rightarrow \text { 2 footed landing). }\end{array}$ \\
\hline 8. & T-agility & Complete t-agility run, facing forwards throughout. \\
\hline 9. & Accelerate-sprint & \begin{tabular}{l} 
10m acceleration to a sprint over finish line. \\
\hline
\end{tabular}
\end{tabular}


The Dragon Challenge Version 1.0 (DC V1.0) has been implemented in schools across Wales. DC V1.0 was designed for use in the field by professionals (sport coaches, PE teachers) and has content and face validity. DCV1.0 does not have criterion validity or reliability data. Empirical work is required to achieve this.

\section{Previous Validation Work that has been completed on DC V1.0:}

Content and face validity - A group of paediatric exercise specialists from Swansea, Glyndwr and Edge Hill University with expertise in physical education, physical competency and physical literacy research were involved in evaluating the content validity of the Dragon Challenge. Content and face validity of the 9 constituent tasks were qualitatively reviewed by a trained researcher. This researcher had $>10$ years' experience of physical competence and movement skill assessment. Internationally recognised experts $(n=5)$ in childhood movement skill, fitness and physical literacy assessment provided guidance for the review process.

Construct validity - Construct validity needs to be gathered on the 9 tasks constituent to the Dragon Challenge in 8-14 years old children.

This research project seeks to investigate the validity and reliability of the Dragon Challenge V1.0 in children aged 8-14 years old using video and accelerometer data. These data will be used to develop and refine the Dragon Challenge V1.0 and create the Dragon Challenge V2.0.

\section{REFERENCES}

- Council of Europe (1983). Testing physical fitness: Eurofit: Experimental Battery: Provisional Handbook. Strasbourg: Council of Europe.

- Folio, M.R., Fewell, R.R. (2000) Peabody Development Motor Scales (PDMS-2). San Antonio, TX: Therapy Skill Builders.

- Henderson, S.E., Sugden, D.A., Barnett, A.L., Smits-Engelsman, C.M. (2010) Movement assessment battery for children - 2. San Antonio, TX: Pearson.

- Janssen, I., Leblanc, A.G. (2010). Systematic review of the health benefits of physical activity and fitness in school-aged children and youth. Int J Behav Nutr Phys Act. 11,7-40.

- Lloyd, M., Saunders, T.J., Bremer, E., Tremblay, M.S. (2014). Long-term importance of fundamental motor skills: A 20-year follow-up study. Adapt Phys Actiy Q, 31:6778.

- Lubans, D.R., Morgan, P.J., Cliff, D.P., Barnett, L.M., Okely, A.D. (2010) Fundamental movement skills in children and adolescents: Review of associated health benefits. Sports Med, 40,1019-35.

- McArdle, W.D., Katch, I. and McArdle, V.L. (2001). Exercise Physiology: Energy, Nutrition and Human Performance. Philadelphia: Lippincott Williams \& Wilkins.

- Robinson, L. E., Stodden, D. F., Barnett, L. M., Lopes, V. P., Logan, S. W., Rodrigues, L. P., \& D'Hondt, E. (2015). Motor competence and its effect on positive developmental trajectories of health. Sports Medicine, 45(9), 1273-1284.

- Stodden, D. F., Goodway, J. D., Langendorfer, S. J., Roberton, M. A., Rudisill, M. E., Garcia, C., \& Garcia, L. E. (2008). A developmental perspective on the role of motor skill competence in physical activity: An emergent relationship. Quest, 60(2), 290306.

- Ulrich, D. A. (2000). Test of gross motor development-2. Austin: Prod-Ed. 
- Whitehead, M. (2010). Physical literacy throughout the life course. London: Routledge Taylor \& Francis Group; 2010.

- Wiart, L., Darrah, J. (2001) Review of four tests of gross motor development. Dev Med Child Neurol, 43:279-85.

\section{AIMS and OBJECTIVES}

The aim of the project is to establish the reliability and validity of the Dragon Challenge V1.0. This will be done using a series of tasks from the Eurofit test battery (Council of Europe, 1983), the Dragon Challenge V1.0, and other motor skill activities from the Test of Gross Movement Development-2 (Ulrich, 2000). There are many objectives, for example: Additionally, this study will seek to use the SlamTracker accelerometer to determine specific signals related to motor performance (as in ethics approval code PG/2014/009), in order to support/further investigate criterion validity.

\section{METHODOLOGY \\ Study Design \\ Participants and settings \\ Instruments and procedures \\ Design and analysis}

One hundred girls and boys from school Years 4, 5, 6, 7, 8 and 9 will be invited to take part in this study. Children will be invited primarily from one primary school and one comprehensive school, with use of past links/communications made with the schools and teachers. Children will complete each of the 9 tasks from the DCV1.0 whilst being video recorded from the frontal, transverse and sagittal planes. These assessments quantify children's motor fitness and fundamental movement skills, including; running, jumping over hurdles, core agility/flexibility, ball throwing, ball catching, ball bouncing, balancing on a bench and balancing on the spot. The Dragon Challenge activities were developed by Swansea and Edgehill Universities and Sport Wales. Observation criteria from Haywood and Getchell (2014) will be used to assess fundamental movement skills in participating children. These data will be compared to DCV1.0 and agreement statistics calculated. This will be repeated twice over 2 weeks to estimate reliability of measures.

The children will also take part in a variety of further motor fitness activities from the Council of Europe European Physical Fitness Test battery (Eurofit) (PG/2014/37) and the Test of Gross Movement Development-2 (Ulrich, 2000). These will provide further measures of object control, locomotor and stability skills which will be used to generate validity data The Eurofit test was devised by the Council of Europe, for children of school age and has been used in many European schools since 1988 (Council of Europe, 1983). Activities such as the flamingo test (stability skill) and $10 \times 5 \mathrm{~m}$ shuttle run (locomotor skill) from this Test battery will be completed. The Test of Gross Movement Development- 2 is a standardized test that measures gross motor abilities in children (Ulrich, 2000). This test includes locomotor skills: run, gallop, jump, slide, hop, and leap, as well as object control skills: striking a stationary ball, stationary dribble, catch, kick, overhand throw, and underhand roll. Other gold standard activities (tests), similar to the activities mentioned above (e.g. Lower Quarter Y Balance Test (YBT-LQ) \& Movement Assessment Battery for Children), may also be used to provide further validity data to investigate criterion validity.

The student's height, sitting height and weight will also be measured so that BMI and maturation offset can be calculated (PG/2014/37). Teachers (Physical education specialist or whoever provides PE lessons) of the participants will provide their perception of the 
child's physical competence (Scale from 1 (very low) to 10 (very high)) as a proxy to investigate predictive validity.

Throughout all activities, participants will be asked to wear the SlamTracker accelerometer (as in ethics approval code PG/2014/009 \& PG14/2014/007). Currently work with SPEC engineers has been completed to develop algorithms to quantify children's movement skills. This study will use the algorithms developed and match them to the video analysis and DCV1.0 data. This will enable the research team to quantify the degree to which movement skills can be assessed using sensors. The pragmatics of children wearing sensors was carried out in (PG/2014/009 \& PG14/2014/007).

At the end of this process construct validity and reliability of the DCV1.0 will be determined. As a result DCV2.0 will be produced.

\section{Experimental Procedures}

Validating the Dragon Challenge

- Year groups: Years 4, 5, 6, 7, 8 and 9

- Duration: $1 \mathrm{hr} 30$ mins for 5 pupils

The study will consist of sessions lasting $1 \mathrm{hr} 30$ mins, with 5 pupils attending each session at a time. The study will take place in the school's sports hall, and/or Swansea University. Active consent will be gained from the head teacher of each school that the children attend, consent will be gained from the parents and assent will be gained from the participant. The participants will self-report their names, date of birth, and home postcode (to calculate WIMD \& SES). Data will be stored on computer and password protected. Teachers (Physical education specialist or whoever provides PE lessons) of the participants will provide their perception of the child's physical competence.

The participants will complete a standardised warm up to increase heart rate, after which they will complete each Dragon Challenge task individually rather than serially. The participants will also take part in further object control, locomotor and stability skills selected from the Eurofit test battery and/or the Test of Gross Movement Development-2. Participants will take part in each activity twice for familiarisation and to ensure quality of video recording. Anthropometric measures will also be taken.

Throughout all activities, participants will be asked to wear the SlamTracker accelerometer (as in ethics approval code PG/2014/009 \& PG14/2014/007). Additionally, during all activities, a video recording device will be used to assess children's movement during the motor fitness activities. Four cameras will be placed along the anteroposterior and mediolateral planes to capture the movements in detail. The cameras will be placed to capture the activities, but will not impede the participants' movements during the motor fitness activities. These video recordings will be used to further analyse the motor fitness/fundamental movement skills of the participants.

\section{Data Analysis Techniques}

- A Pearson's product moment correlation will be used to analyse concurrent validity.

- Limit's of agreement will be sued to assess agreement between measures

- Factor analysis or Principal Component analysis (PCA)

- Confirmatory Factor Analysis (CFA) 
- To further assess construct validity, age and gender differences will be explored using independent t-tests and chi square, for DC Score, Time, Technique (process) and Outcome (product) variables, as well as in the individual criterion.

- Raw acceleration data from the SlamTrackers (as in ethics approval code PG/2014/009) will be analysed using an algorithm developed from a previous study (PG/2014/009 \& PG14/2014/007). The trace from the accelerometer, which gives acceleration in terms of ' $\mathrm{g}$ ' over the time period, is split into sections for clarity and ease of analysis. The data is then converted into the frequency domain by the Fast Fourier transform. The Fast Fourier transform involves taking a continuous function and representing it as a number of sine and cosine waves. It provides information about the harmonic content of a signal so that conclusions about the activity being performed can be made. Data will be analysed using MatLab, with time and frequency features from the raw acceleration trace extracted.

- Video recordings will be used to analyse and compare the motor fitness activities and also to compare/confirm assessor (rater) scores.

\section{Storage and Disposal of Data and Samples}

All data will be kept private and confidential, hard copies of information will be kept in a locked cabinet in a secure office and any personal information will be changed into a number rather than a name. All files on a computer will be password protected and files with personal details will only be accessible by the research team. Any and all video recorded data will be kept separate from any identifying documents and will be managed under the Data Protection Act 1998. The video recorded data will be kept on an external memory drive which will be password protected at all times. After analysis, the video recorded data will be deleted, and alternatively, any video recorded data not used for analysis will be destroyed. Teachers will not have access to any individual data or nonanonymised data, but anonymised group data will be provided to teachers on their request. Lead professional for City and County of Swansea and from Mid \& West Wales PLPS who are part of the research team (Wendy Anderson, Sarah McCoubrey, Helen John \& Kirsty Edwards) will have access to the data.

\section{LOCATION OF THE PREMISES WHERE THE RESEARCH WILL BE CONDUCTED.}

The project will take place in School sports hall or in the University Sportshall. The research team, member(s) of PLPS group, and a member(s) of City and County of Swansea sport and recreation team will all be present. School teachers and/or teaching assistants will also be present, and a qualified first aider (Swansea University sport hall staff, or school sports hall attendant, or member of PLPS group/AYP team, or qualified member of the research team).

\section{PARTICIPANT RISKS AND DISCOMFORTS}

The students will not be put under any greater risk than what they would face when during physical activity during sport dance or play. However, there is a possibility that whilst taking part children may get injured, feel sick or faint. First aid trained staff will be available during any activity. Weight will be measured privately and sensitively. All lead researchers will have a DBS check and no researcher will be on their own with any individual child.

DBS Certificate Number for Lead Researcher (Richard Tyler) - 001464235079

Other evidence for DBS certificates can be obtained from the Project Director. 


\section{INFORMATION SHEET AND INFORMED CONSENT}

The submission should be specific about the type of consent that will be sought, which should be indicated below:

- Have you included a Participant Information Sheet for the participants of the study? YES/NO

- Have you included a Participant Consent Form for the participants of the study? YES/NO

\section{COMPUTERS}

- Are computers to be used to store data?

$$
\text { YES/AO }
$$

- If so, is the data registered under the Data Protection Act?

$$
\text { YES/NO }
$$

\section{STUDENT DECLARATION}

Please read the following declarations carefully and provide details below of any ways in which your project deviates from these. Having done this, each student listed in section 2 is required to sign where indicated.

- "I have ensured that there will be no active deception of participants.

- I have ensured that no data will be personally identifiable.

- I have ensured that no participant should suffer any undue physical or psychological discomfort

- I certify that there will be no administration of potentially harmful drugs, medicines or foodstuffs.

- I will obtain written permission from an appropriate authority before recruiting members of any outside institution as participants.

- I certify that the participants will not experience any potentially unpleasant stimulation or deprivation.

- I certify that any ethical considerations raised by this proposal have been discussed in detail with my supervisor.

- I certify that the above statements are true with the following exception(s):"

Student/Researcher signature: (include a signature for each student in research team) Date:

\section{SUPERVISOR'S DECLARATION}

In the supervisor's opinion, this project (delete those that do not apply):

- "Does not raise any significant issues.

- $\quad$ Raises some ethical issues, but I consider that appropriate steps and precautions have been taken and I have approved the proposal.

- Raises ethical issues that need to be considered by the Departmental Ethics Committee.

- $\quad$ Raises ethical issues such that it should not be allowed to proceed in its current form."

Supervisor's signature:

Date: 
ETHICS COMMITTEE DECISION (COMMITTEE USE ONLY)

ETHICAL APPROVAL: REC PG/2014/39

GRANTED/REJECTED

The ethical issues raised by this project have been considered by the A-STEM Ethical Advisory Committee.

Please refer to the summary comments noted in the decision email sent to the project lead/supervisor. Please ensure that you take account of these comments.

Where necessary, please prepare a revised submission that should be shown to your supervisor (for student applications) and then resubmitted to the A-STEM Ethical Advisory Committee for reconsideration.

Application number

Date:

Signed:

(Chair, A-STEM Ethical Advisory Committee) 


\section{Applied Sports Technology Exercise and Medicine Research Centre (A-STEM) \\ Sport and Health Portfolio, College of Engineering}

\section{PARENT INFORMATION SHEET}

(Version 1.0, Date:_/_/20_)

Project Title:

The validity and reliability of the Dragon Challenge V1.0 in measuring physical competency in children aged 8-14 years.

\section{Contact Details:}

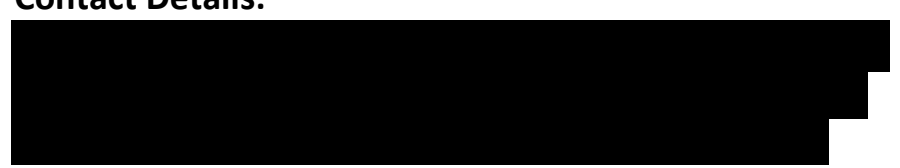

\section{Please read the information below carefully before deciding whether to consent} for your child's participation.

\section{Invitation Paragraph}

Children in your child's class/year at school have been invited to take part in a new study that will look at how good the Dragon Challenge is at measuring physical competency (how well the children can perform the fundamental movement skills needed in all sports, dance and physical activities). Our aim is to assess the validity and reliability of the Dragon Challenge V1.0 at measuring physical competency.

\section{What is the purpose of the study?}

The purpose of the study is to investigate the validity and reliability of the Dragon Challenge V1.0 in measuring physical competency in children aged 8-14 years. The study will also use the SlamTracker accelerometer, which is a small sensor in a device similar to a watch that measures your child's movement.

\section{Why has my child been chosen?}

Children in your child's class have been invited to take part in the study. During the study, if your child does not feel happy about anything that they are asked to do, they can stop at any time, without fear of penalty. If you need any more information about the study then please contact any member of the team on the details above.

\section{What will happen to your child if they take part?}

Your child will participate in a number of fun physical activities which will measure movement skills. These involve moving the body in any direction from one point to another, balancing the body in one place or while in motion, and handling or controlling objects with the hand or foot. Sport Wales, in partnership with Swansea University, Glyndwr University and Edge Hill University, have carefully constructed a single practical assessment "Dragon Challenge V1.0" that aims to assess many of these movement skills. During the Dragon Challenge, your child and the other children invited from their class will perform 9 discrete tasks that require single 
or combinations of skills in order to achieve a goal. These tasks involve running, jumping over hurdles, balancing, core agility/flexibility, ball throwing, ball catching and ball bouncing. These will all be completed separately as well as in a timed circuit. Your child will also be asked to take part in similar activities to those in the Dragon Challenge V1.0; this is to see if they perform these activities as well as they perform in the Dragon Challenge activities.

During all of the physical activities, all of the children will be asked to wear a small sensor (called a SlamTracker accelerometer) on their wrist and ankle, the device is small and is similar to a watch. This device will measure how fast your child moves forwards and backwards, side to side and up and down. Your child will also have weight, height and sitting height measurements. Measures of body weight are taken privately behind a screen, there will always be other children in the room or next to the screen but they will not be party to the results or be able to view the measures and no results are shared with the rest of the class.

All the activities during the study are educational for the children's enjoyment and not as a competition. With your permission, a video camera will also be used to capture the activities the children are performing; this will allow us to further investigate the reliability and validity of the activities and results.

\section{What are the possible disadvantages of taking part?}

Taking part in the Dragon Challenge and other measures of movement performance poses no greater risk than a child participating in physical activity in sport, dance or play. However, in the unlikely event that a child feels unwell a qualified first aider will always be available during the study sessions.

\section{What are the possible benefits of taking part?}

The activities will be active and educational for the children and they will get to take part in a variety of different activities. They will be able to find out a lot about their physical skills.

\section{Will my child taking part in the study be kept confidential?}

All the data we collect from your child will be kept private and confidential; the children's names will be changed to numbers. Any hard copies of data will be kept in a secure office and computer files with any personal information will be password protected. The data obtained will only be looked at by responsible individuals of the research team. All of the recorded videos will be securely stored and will not be shared publicly. These will be deleted once analysed.

\section{What if I have any questions?}

If you have any questions about what is written above or anything to do with the study please don't hesitate to contact me or anyone from the research team (see contact details above). If after the study you are concerned about how any aspect of the research was conducted please contact the Chair of the College Ethics Committee, Professor Mike McNamee (m.j.mcnamee@swansea.ac.uk). 


\section{Applied Sports Technology Exercise and Medicine Research Centre (A-STEM) \\ Sport and Health Portfolio, College of Engineering}

\section{PARENT CONSENT FORM}

(Version 1.0, Date: ____/20__

\section{Project Title:}

The validity and reliability of the Dragon Challenge V1.0 in measuring physical competency in children aged 8-14 years.

\section{PLEASE RETURN THIS FORM TO SCHOOL TO CONSENT FOR YOUR CHILD TO TAKE PART IN THE PROJECT.}

\section{Contact Details:}

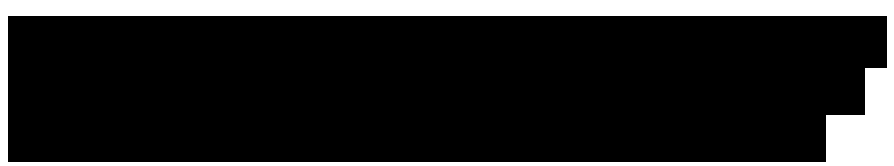

\section{Please INITIAL each box below}

1. I confirm that I have read and understood the information sheet dated __ 20_ (Version number 1.0) for the above study and have had the opportunity to ask questions.

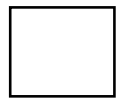

2. I am happy for my child to participate in the Dragon Challenge and similar activities, and wear the sensor that tracks movement.

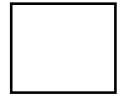

3. I understand that my child's participation is voluntary and that I am free to withdraw my child at any time, without giving any reason, without their medical care or legal rights being affected.

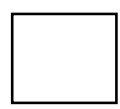

4. I understand that sections of the data obtained may be looked at by responsible individuals from the Swansea University or from regulatory authorities where it is relevant to the children's taking part in research

5. I am happy for the activities to be video recorded for academic use ONLY. I agree to allow my child to take part in the above study.

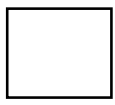

Name of Participant (child)

Name of Parent/Guardian

Researcher

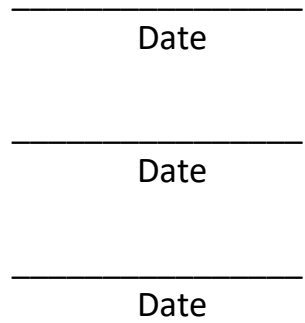

Date
Signature

Signature

Signature 


\title{
Applied Sports Technology Exercise and Medicine Research Centre (A-STEM) \\ Sport and Health Portfolio, College of Engineering
}

\section{HEADTEACHER INFORMATION SHEET}

\author{
(Version 1.0, Date: ___/20_)
}

Project Title:

The validity and reliability of the Dragon Challenge V1.0 in measuring physical competency in children aged 8-14 years.

Contact Details:

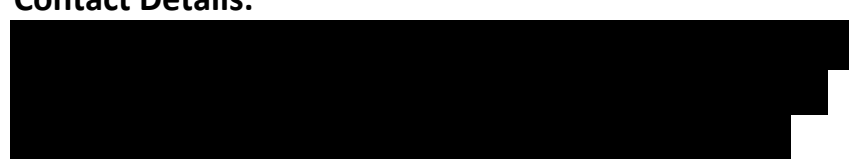

\section{Invitation Paragraph}

Children from your school have been invited to take part in a new study that will look at how valid and reliable the Dragon Challenge is at measuring physical skills. The data we collect from all of the children taking part in the study will help us assess the validation and reliability of the Dragon Challenge V1.0 in measuring physical competency.

\section{What is the purpose of the study?}

The purpose of the study is to investigate the validity and reliability of the Dragon Challenge V1.0 in measuring physical competency in children aged 8-14 years. The study will also use a small sensor to quantify movement. We are interested to see if physical skills are related to children's health. This will further help to decide how best to help children become healthier and physically active in the future and throughout their life.

\section{Why have I been chosen?}

Children from your school/class have been invited to take part in the study. During the study, if the children do not feel happy about anything that they are asked to do, they can stop at any time, without fear of penalty. If you need any more information about the study then please contact any member of the team on the details above.

\section{What will happen to the children if they take part?}

The children will attend a session at Hafod School and/or at Swansea University. This will involve around $1 \mathrm{hr} 30 \mathrm{~min}$ sessions ( 5 children per session) of fun physical activities which will measure children's aspects of physical competence (this Challenge aims to assess many fundamental movement skills: locomotion - skills that involve moving the body in any direction from one point to another, stability - skills that involve balancing the body in one place or while in motion, \& object control - skills that involve handling or controlling objects with the hand, foot or an implement). During the Dragon Challenge, the children will perform 9 discrete tasks that require single or combinations of skills in order to achieve a goal. These tasks involve running, jumping over hurdles, balancing, core agility/flexibility, ball throwing, ball catching and ball bouncing. These will all be completed separately as well as in a timed circuit. The children will also be asked to take part in similar activities to those in the Dragon Challenge 
V1.0; this is to see if they perform these activities as well as they perform in the Dragon Challenge activities.

During all of the physical activities, all of the children will be asked to wear the SlamTracker accelerometer (a small sensor) on their wrist and ankle. This device will measure how fast each child moves forwards and backwards, side to side and up and down. The children will also have weight, height and sitting height measurements recorded, taken by a member of the research team listed. Measures of body weight are taken privately in a separate room or behind a screen, there will always be other children in the room or next to the screen but they will not be party to the results or be able to view the measures and no results are shared with the rest of the children, however, the children will not have to have them taken if they do not want to. All activities will be no harder than the children would do during school PE lessons.

All the activities during the study are aimed to educate and for the children's enjoyment, and not as a competition. With your permission, a video camera will also be used to capture the activities the children are performing; this will allow us to further investigate the reliability and validity of the activities and results.

\section{What are the possible disadvantages of taking part?}

Taking part in the Dragon Challenge and other physical activities poses no greater risk than a child participating in school physical education lessons or during physical activity in sport, dance or play. However, in the unlikely event that a child feels unwell, there will be people monitoring the children during all parts of the activities, and the children's teachers will remain present at all times. A qualified first aider will always be present during the study sessions.

\section{What are the possible benefits of taking part?}

The activities will be active and educational for the children and they will get to take part in a variety of different activities that they might not have taken part in before. They will be able to find out a lot about their physical skills.

\section{Will my taking part in the study be kept confidential?}

All the data we collect from the children will be kept private and confidential; the children's names will be changed to numbers. Any hard copies of data will be kept in a secure office and computer files with any personal information will be password protected. The data obtained will only be looked at by responsible individuals of the research team, or from regulatory authorities where it is relevant to your child's participation in the research. All of the recorded videos will be securely stored and will not be shared publicly, and will be deleted once analysed. Any videos that are not used for analysis will be destroyed.

\section{What if I have any questions?}

If you have any questions about what is written above or anything to do with the study please don't hesitate to contact me or anyone from the research team (see contact details above). If after the study you are concerned about how any aspect of the research was conducted please contact the Chair of the College Ethics Committee, Professor Mike McNamee (m.j.mcnamee@swansea.ac.uk). 


\title{
Applied Sports Technology Exercise and Medicine Research Centre (A-STEM) \\ Sport and Health Portfolio, College of Engineering
}

\section{HEADTEACHER CONSENT FORM}

\author{
(Version 1.0, Date: ____/20_)
}

\section{Project Title:}

The validity and reliability of the Dragon Challenge V1.0 in measuring physical competency in children aged 8-14 years.

\section{Contact Details:}

\section{Please INITIAL each box below}

1. I confirm that I have read and understood the information sheet dated __ 20 _ (Version number 1.0) for the above study and have had the opportunity to ask questions.

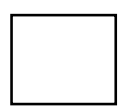

2. I understand that the children's participation is voluntary and that I am free to withdraw my pupils at any time, without giving any reason, without their medical care or legal rights being affected.

3. I understand that sections of the data obtained may be looked at by responsible individuals from the Swansea University or from regulatory authorities where it is relevant to the children's taking part in research.

4. I am happy for the activities to be video recorded for academic use ONLY.

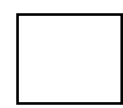

I agree to allow the pupils in my school to take part in the above study.

Name of School

Name of Headteacher

Researcher

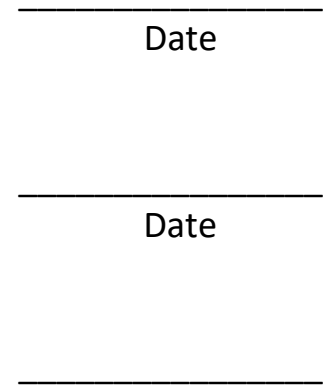

Date
Signature

Signature 


\title{
PARTICIPANT INFORMATION SHEET
}

\section{Project Title:}

\author{
(Version 1.0, Date:___/20_)
}

The validity and reliability of the Dragon Challenge V1.0 in measuring physical competency in children aged 8-14 years.

Contact Details:

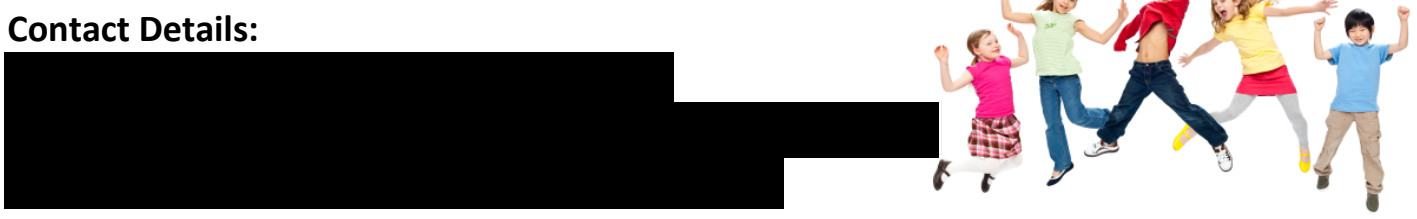

You have been invited to take part in a Swansea University study. You have been chosen because you are between the ages of 8-14 years old and go to a school in Wales.

In this study you have the chance to take part in the Dragon Challenge, and other similar activities, that look at how well you can perform tasks that are needed in all sports, dance and physical activity. Physical activity is any movement that requires your body to work harder than it does whilst sitting, or resting. The sessions will involve lots of fun physical activities that you may not have tried before which should be really enjoyable. The Dragon Challenge involves running, jumping over hurdles, balancing, core agility/flexibility, ball throwing, ball catching and ball bouncing. These will be done separately as well as done in a circuit and should be really fun. The other activities that you will be asked to take part in will be similar to the ones in the Dragon Challenge.

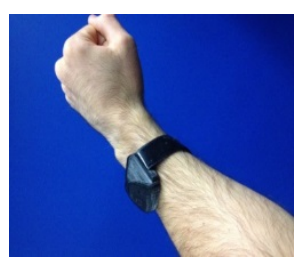

You will also get asked to wear our BRAND NEW devices, and use the NEWEST technology to measure how fast you move forwards and backwards, side to side and up and down. This will be worn on your wrist and ankle. You will also be video recorded during all of the physical activities. Don't worry this is just to help us see how well you have done and help us see if the Dragon Challenge needs changing. None of the activities are a competition.

You will not be forced to do any of the activities and can stop at any time without fear of penalty or having to worry about being in trouble.

If you have any questions please ask.

THANK YOU. 


\title{
Applied Sports Technology Exercise and Medicine Research Centre (A-STEM) \\ Sport and Health Portfolio, College of Engineering
}

\section{PARTICIPANT ASSENT FORM}

\author{
(Version 1.0, Date: ___/20__)
}

Project Title:

The validity and reliability of the Dragon Challenge V1.0 in measuring physical competency in children aged 8-14 years.

\section{Contact Details:}

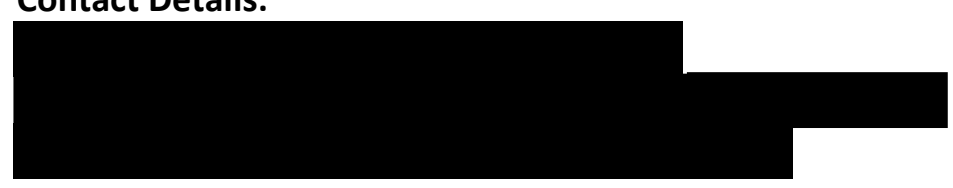

\begin{tabular}{|l|c|c|}
\hline & $\begin{array}{c}\text { Please look at the following statements and put your } \\
\text { initials if you agree. Example Sarah Jones: SJ }\end{array}$ & \\
\hline I have read the Participant Information Sheet & \\
\hline I understand what I will be doing if I take part & \\
\hline
\end{tabular}

